\title{
Maßnahmen zur Reduzierung von Unfällen mit Straßenbahnen
}

Andreas Grießbach

Nadja Seiler

Ulrich Brannolte

Uwe Plank-Wiedenbeck

Jean Emmanuel Bakaba

Unfallforschung

Jörg Ortlepp

der Versicherer 


\section{Maßnahmen zur Reduzierung von Unfällen mit Straßenbahnen}

Dipl.-Ing. Andreas Grießbach Dipl.-Wirt. Ing. (FH) Nadja Seiler Univ. Prof. (em.) Dr.-Ing. Ulrich Brannolte Prof. Dr.-Ing. Uwe Plank-Wiedenbeck

Dr.-Ing. Jean Emmanuel Bakaba Dipl.-Ing. Jörg Ortlepp

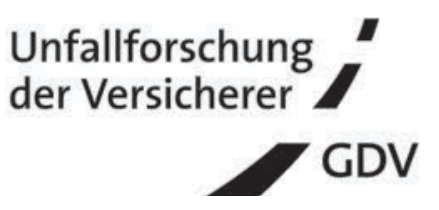




\section{Impressum}

Gesamtverband der Deutschen Versicherungswirtschaft e. V. Unfallforschung der Versicherer

Wilhelmstraße 43/43G, 10117 Berlin

Postfach 0802 64, 10002 Berlin

E-Mail: unfallforschung@gdv.de

Internet: www.udv.de

Facebook: www.facebook.com/unfallforschung

Twitter: @unfallforschung

YouTube: www.youtube.com/unfallforschung

ISBN-Nr.: 978-3-939163-65-7

Redaktion: Dr.-Ing. J. Emmanuel Bakaba, Dipl.Ing. Jörg Ortlepp

Bildnachweis: UDV und siehe Quellenangaben

Erschienen: 06/2016 


\section{Maßnahmen zur Reduzierung von Unfällen mit Straßenbahnen}

Bearbeitet durch:

Bauhaus-Universität Weimar Professur Verkehrssystemplanung

Dipl-Ing. Andras Grießbach Dipl.-Wirt. Ing. (FH) Nadja Seiler

Fachlich Betreut von:

Univ. Prof. (em.) Dr.-Ing. Ulrich Brannolte Prof. Dr.-Ing. Uwe Plank-Wiedenbeck

VSP

Professur

Verkehrssystemplanung

Bauhaus-Universität Weimar

Bei der UDV betreut von:

Dr.-Ing. J. Emmanuel Bakaba

Dipl.-Ing. Jörg Ortlepp

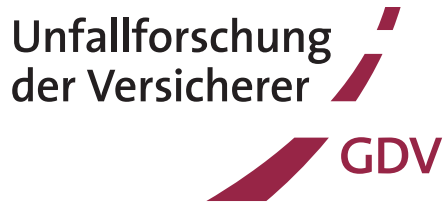





\section{Autorenreferat}

Mit dem Forschungsvorhaben "Maßnahmen zur Reduzierung von Unfällen mit Straßenbahnen" wurden auf Basis umfassender polizeilicher Unfalldaten belastbare Erkenntnisse zur Verkehrsinfrastruktur bei Unfällen mit Straßenbahnen gewonnen und Empfehlungen daraus abgeleitet.

Es wurden dazu im Wesentlichen zwei methodische Ansätze kombiniert: In einem ersten Schritt erfolgte für 58 deutsche Städte mit Straßenbahnbetrieb von den dafür vorliegenden Unfalldaten über drei Kalenderjahre eine statistische Auswertung (makroskopische Analyse). Als Basis dafür lagen insgesamt über 4.000 Unfälle mit Personenschaden unter Beteiligung von Straßenbahnen vor.

Ergänzt wurden diese Unfalldaten um straßenräumliche, typisierte Merkmale, sodass die statistische Auswertung auf diese Typisierungsmerkmale ausgeweitet werden konnte.

Als Ergebnis wurden zahlreiche Diagramme erstellt und erläutert. Die Verteilungen der Unfall- und Typisierungsmerkmale zeigen Zusammenhänge auf, aus denen für Planung und Betrieb von Straßenbahnverkehrsanlagen besondere Handlungsschwerpunkte in Abhängigkeit unterschiedlicher Verkehrsanlagen und nach Art der Verkehrsbeteiligung abgeleitet werden.

Für definierte Querschnittstypen und für die straßenräumliche Entwurfssituation wurde für fünf ausgewählte Städte eine netzfeine Untersuchung der Merkmalsverteilungen durchgeführt. Als Ergebnis liegen differenzierte längenbezogene Unfallkenngrößen und strukturelle Unterschiede in Abhängigkeit unterschiedlicher Querschnittstypen und RASt-Entwurfssituationen vor.

Im zweiten Schritt des Forschungsvorhabens wurden 32 auffällige Unfallkonzentrationen, auf der Basis von Ortsbegehungen vertieft untersucht (mikroskopische Analyse).

In Anlehnung an Prozeduren eines Sicherheitsaudits für Straßen wurden im Bestand sicherheitsrelevante Defizite der Straßenräume erhoben. Charakteristische Sicherheitsdefizite konnten nach Art der Verkehrsanlage aufgestellt werden. Diese können bei Planung und Evaluierung von Straßenbahnanlagen unterstützend herangezogen werden.

Neben der Erstellung konkreter Handlungsempfehlungen zur sicheren Gestaltung von Straßenbahnverkehrsanlagen wurde in Anlehnung an die "Empfehlungen für das Sicherheitsaudit von Straßen" eine ergänzte Prüfliste erarbeitet, die thematisch geordnet typische Sicherheitsdefizite bei der Planung und im Betrieb von Straßenbahnverkehrsanlagen auflistet. Mit dieser erstellten Liste ist es möglich Sicherheitsuntersuchungen für Straßenbahnverkehrsanlagen bei Planungen und im Bestand durchzuführen. 


\section{Abstract}

Within the research project "measures to reduce tramway accidents" reliable scientific conclusions regarding tramway- and road infrastructure could be set up based on data of more than 4.000 tramway-accidents recorded by the police on common standard.

There were essentially combined two methodological approaches:

On the one hand evaluations on statistical analysis (macroscopic analysis) were carried out for 58 German cities with tramway networks. The input data were data of accidents with personal injuries related on three years complemented by acquired data concerning road space e.g. characteristic of junctions, cross sections and tram stops.

As a result, numerous diagrams were created and get explained. The statistical distributions of the accident-characteristics and infrastructure-characteristics point out relationships and special priorities of action according to different transport infrastructure and types of road use significant from the beginning of the preliminary planning up to possibilities for change during the operating.

For defined types of cross sections and road space designs an in-depth study of networks of five German cities get part of the research project.

Finally differentiated length-related accident figures and structural differences depending on different types of cross sections and road space designs were figured out.

On the other hand 32 conspicuous accident concentrations, deepened on the basis of on-site inspections were analysed (microscopic analysis).

Based on procedures of road safety audits deficits of junctions and cross sections were recorded. Characteristic deficits could be set up by types of infrastructure useful in the planning and evaluation of tramway systems.

In addition to specific recommendations for tramway infrastructure a checklist based on the "Recommendations for road safety audits" get expanded. These check list reflects typical safety deficits of tramway infrastructure. Therewith it is possible to carry out road safety evaluations of tramway facilities in the planning and operation of tramway transport infrastructure. 


\section{Inhaltsverzeichnis}

Autorenreferat

Abstract

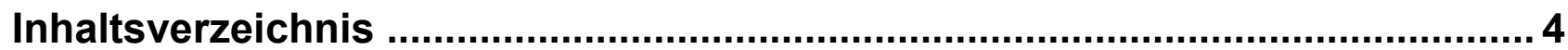

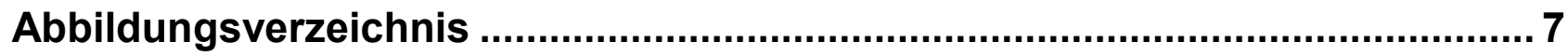

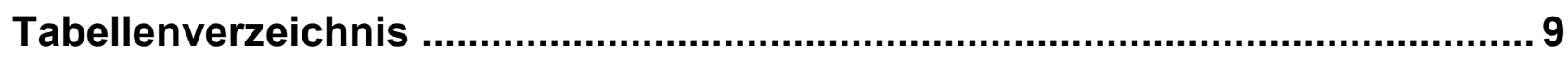

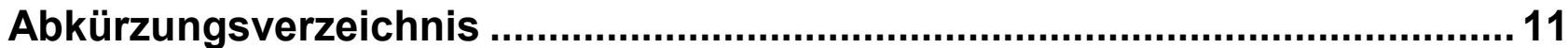

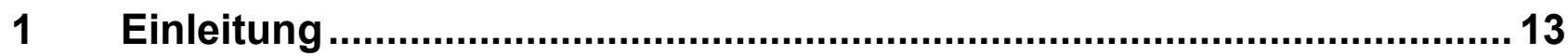

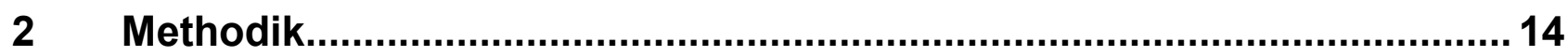

2.1 Abgrenzung des Untersuchungsgegenstandes.........................................14

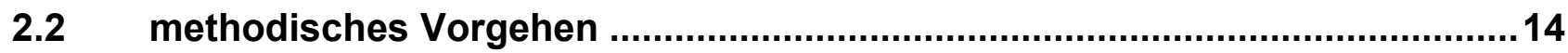

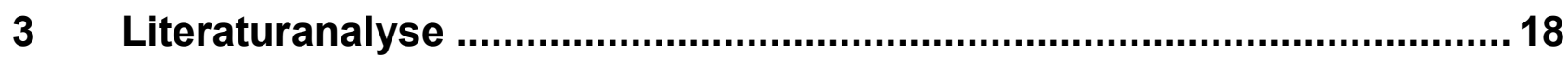

3.1 Grundlagen zur Gestaltung von Straßenbahninfrastruktur............................18

3.1.1 Straßenbahninfrastruktur im städtischen Raum ............................................... 18

3.1.2 Netzelemente der Straßenbahninfrastruktur ................................................ 19

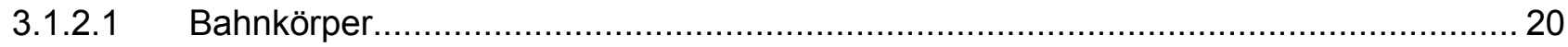

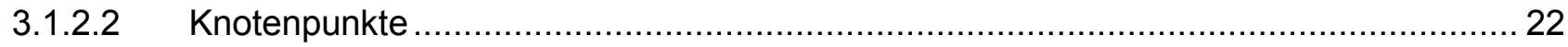

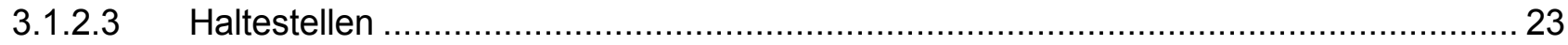

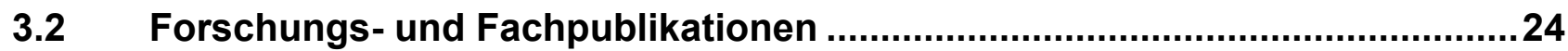

3.2.1 Sicherheitsuntersuchungen an Haltestellen des ÖPNV ......................................25

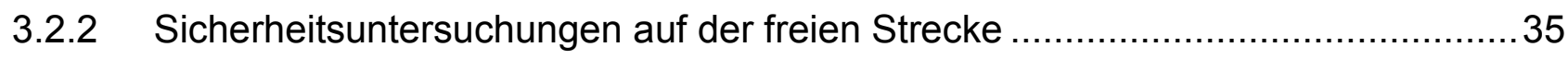

3.3 Rechtliche Vorgaben, Regelwerke und Richtlinien .......................................39

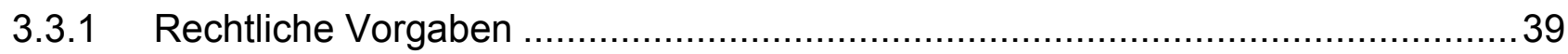

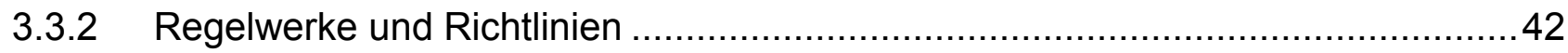

4 Sicherheit von Straßenbahnen im Vergleich zu anderen ausgewählten innerstädtischen Verkehrsmitteln: bundesweite Analyse ...................... 57 
5 Sicherheitsanalyse von Straßenbahnnetzen in ausgewählten Städten . 62

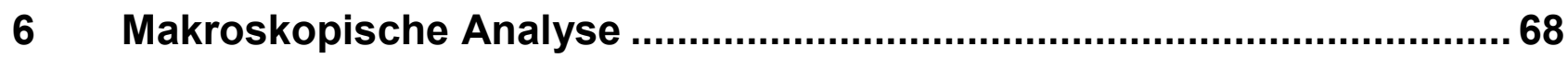

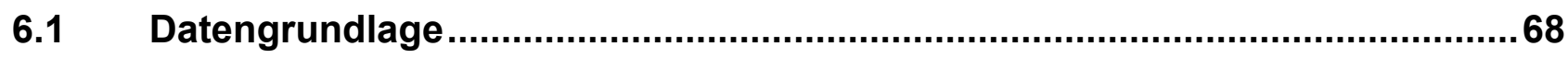

6.1.1 Art und Umfang der zugrunde gelegten Daten (Datenkollektive) .........................68

6.1.2 Unfalldaten: Erfassung, Aufbereitung und besondere Hinweise

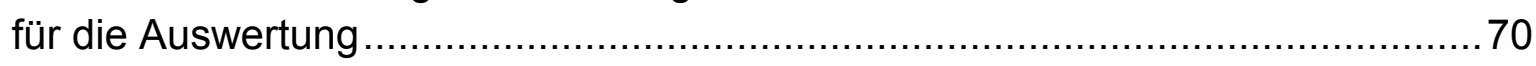

6.1.3 Netzdaten und straßenräumliche Merkmale: Erfassung, Aufbereitung und besondere Hinweise für die Auswertung ........................................................76

6.2 Ergebnisse der makroskopischen Analyse .................................................79

6.2.1 Ergebnisse zum Unfallgeschehen Allgemein und nach Art der Verkehrsanlage .. 80

6.2.2 Sicherheitsbewertung nach Art der Verkehrsteilnahme als Hauptverursacher ..... 89

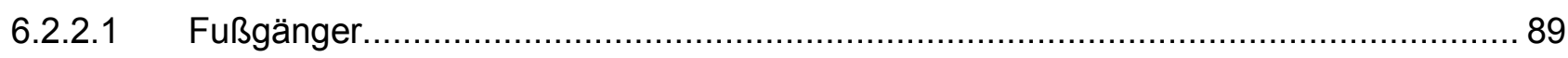

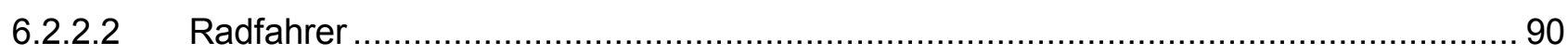

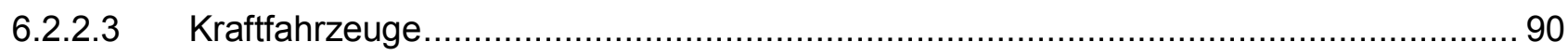

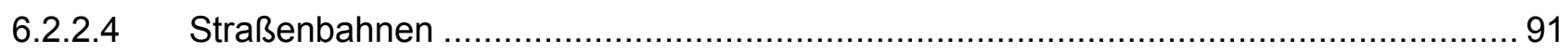

6.3 Zusammenfassung der makroskopischen Analyse ......................................92

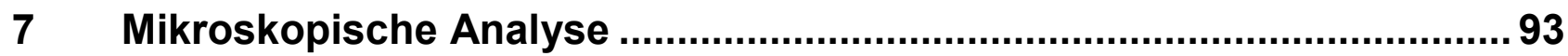

7.1 Kriterien für die Auswahl der zu untersuchenden Verkehrsanlagen...............93

7.2 Datengrundlage, Ortsbesichtigungen, Sicherheitsaudits, Datenauswertung und Dokumentation .........................................................98

7.3 Ergebnisse der mikroskopischen Analyse ...................................................99

7.4 Zusammenfassung der mikroskopischen Analyse .......................................106

8 Zusammenfassung der Projektergebnisse und Empfehlungen ............ 107

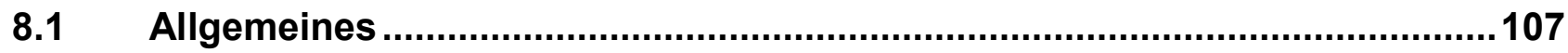

8.2 Sicherheit nach der Art der Verkehrsbeteiligung .......................................107

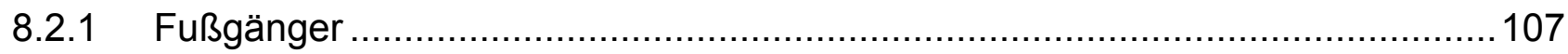

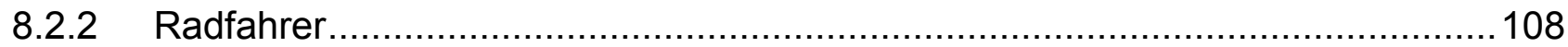

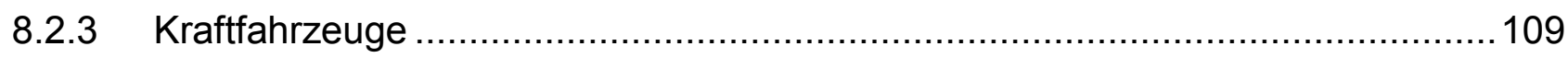

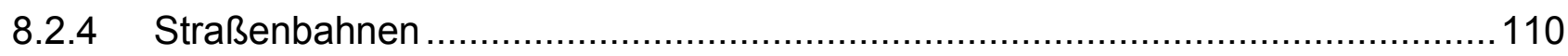


8.3 Sicherheit nach der Art der Verkehrsanlage ............................................110

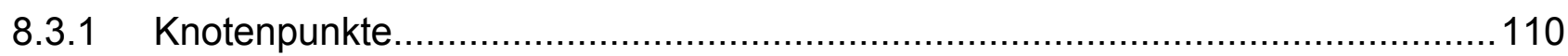

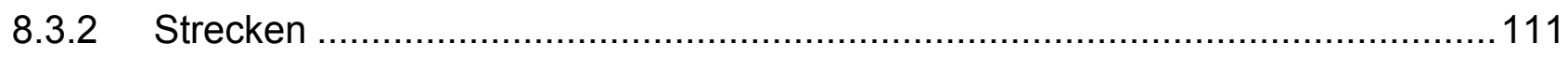

8.4 Sicherheitshinweise zur Implementierung in Regelwerken ..........................112

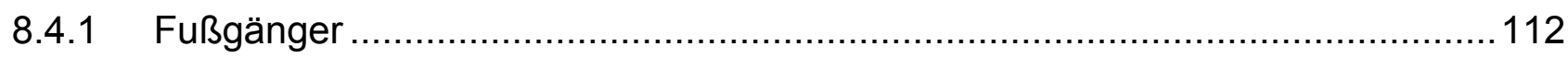

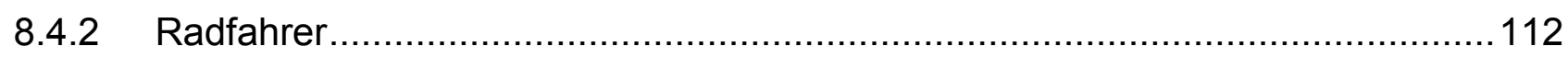

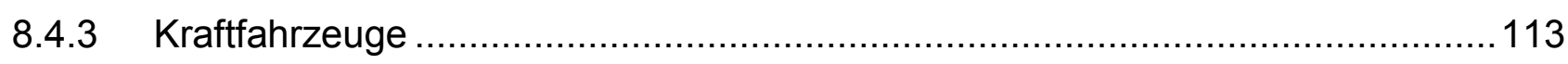

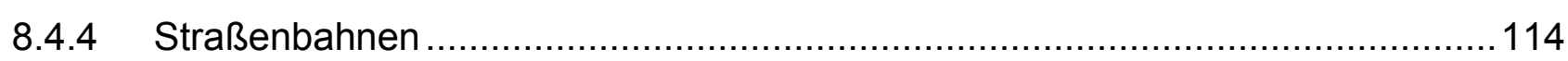

8.4.5 ergänzende Sicherheitshinweise in der ESAS-Defizitcheckliste ........................115

$9 \quad$ Zusammenfassung und Fazit............................................................ 116

Literaturverzeichnis ......................................................................... 123

Anlagen

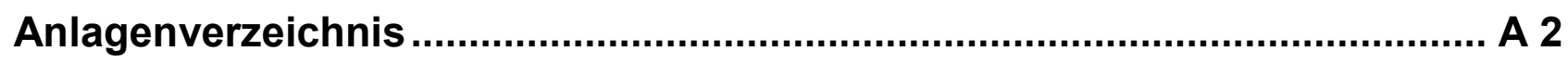




\section{Abbildungsverzeichnis}

Abbildung 4-1: Verkehrsmittelvergleich nach Unfallschwere [Getötete pro 1.000 Unfälle mit Personenschaden oder schwerwiegendem Sachschaden]

Abbildung 4-2: Verkehrsmittelvergleich nach Unfallschwere [1.000 € pro Unfall mit Personenschaden oder schwerwiegendem Sachschaden] .

Abbildung 4-3: fahrleistungsbezogener und beförderungsleistungsbezogener

Verkehrsmittelvergleich

Abbildung 5-4: Unfallkostendichte nach Querschnittstyp, alle $U_{\text {Strab }}(P)$

Abbildung 5-5: Unfallkostendichte nach Querschnittstyp, nur $U_{\text {Strab }}(P)$ außerhalb v. Knotenpunkten

Abbildung 5-6: Unfallkostenrate nach Querschnittstyp, alle $\bigcup_{\text {Strab }}(P)$

Abbildung 5-7: Unfallkostenrate nach Querschnittstyp, nur $U_{\text {Strab }}(P)$ außerhalb v.

Knotenpunkten

Abbildung 5-8: Unfalltyp nach Querschnittstyp, alle $\cup_{\text {Strab }}(P)$

Abbildung 5-9: Unfalltyp nach Querschnittstyp, nur $\cup_{\text {Strab }}(P)$ außerhalb v. Knotenpunkten ... 64

Abbildung 5-10: Hauptverursacher nach Querschnittstyp, alle $U_{\text {Strab }}(P)$

Abbildung 5-11: Hauptverursacher nach Querschnittstyp, nur $U_{\text {Strab }}(P)$ außerhalb v. Knotenpunkten

Abbildung 5-12: Unfallkostendichte nach Entwurfssituation, alle $U_{\text {Strab }}(P)$

Abbildung 5-13: Unfallkostendichte nach Entwurfssituation, nur $U_{\text {Strab }}(P)$ außerhalb v.

Knotenpunkten

Abbildung 5-14: Unfallkostenrate nach Entwurfssituation, alle $\bigcup_{\text {Strab }}(P)$

Abbildung 5-15: Unfallkostenrate nach Entwurfssituation, nur $U_{\text {Strab }}(P)$ außerhalb v. Knotenpunkten

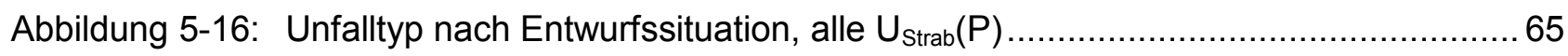

Abbildung 5-17: Unfalltyp nach Entwurfssituation, nur $U_{\text {Strab }}(P)$ außerhalb v. Knotenpunkten ...... 65

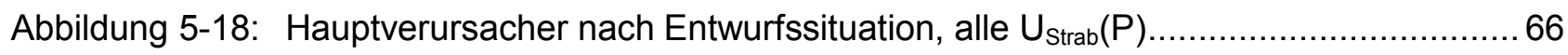

Abbildung 5-19: Hauptverursacher nach Entwurfssituation, nur $U_{\text {Strab }}(P)$ außerhalb v. Knotenpunkten

Abbildung 6-20: Ermittlung der abschnittsfeinen Straßenbahnquerschnittsbelastung am Beispiel Hannover

Abbildung 6-21: berechnete angepasste Unfallkostensätze ............................................. 79

Abbildung 6-22: Knotenpunktabstände (Summenkurve) .................................................. 80

Abbildung 6-23: Ordnungsnummer der Unfallbeteiligung nach Art der Beteiligung .................... 81

Abbildung 6-24: Art der Verkehrsbeteiligung der Verunglückten nach Unfallfolge..................... 81

Abbildung 6-25: Art der Verkehrsbeteiligung der Getöteten und Schwerverletzten nach Lage im Netz. 
Abbildung 6-26: Hauptverursacher nach Unfallschwere ................................................ 82

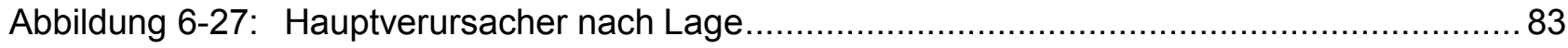

Abbildung 6-28: Anteil der Knotenpunktunfälle nach Knotenpunktart ..................................... 84

Abbildung 6-29: Hauptverursacher nach Knotenpunktart................................................... 85

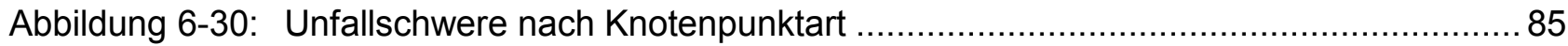

Abbildung 6-31: Unfallkostenrate nach Knotenpunktart und Straßenbahnverkehrsstärke........... 86

Abbildung 6-32: Knotenpunktunfälle nach der von der Straßenbahn befahrenen Querschnittstypen am Knotenpunkt ................................................... 87

Abbildung 6-33: Hauptverursacher nach von der Straßenbahn befahrenen Querschnittstypen an Knotenpunkten .................................................. 87

Abbildung 6-34: Unfallschwere von Streckenunfällen nach Querschnittstypen ........................ 88

Abbildung 8-35: Prinzipskizze Haltestelle in Insellage mit Mittelbahnsteig ............................. 113

Abbildung 8-36: Alternativlösung Prinzipskizze Haltestelle in Insellage mit Mittelbahnsteig....... 113 


\section{Tabellenverzeichnis}

Tabelle 3-1: Mindestanforderungen für Seitenraumbreiten 18

Tabelle 3-2: $\quad$ Mindestraumbreiten zur Integration von Straßenbahninfrastruktur unter Beachtung der Straßenraumnutzung 19

Tabelle 3-3: $\quad$ Führung des Bahnkörpers im Straßenraum

Tabelle 3-4: $\quad$ Mindestraumbreiten zur Integration von Straßenbahninfrastruktur unter Beachtung der Straßenraumnutzung

Tabelle 3-5: Knotenpunktgrundformen in Zusammenhang mit Straßenbahnverkehrsanlagen. 22

Tabelle 3-6: Haltestellenformen des schienengebundenen ÖV 24

Tabelle 3-7:

Gefährdungspotentiale nach Haltestellenformen

Tabelle 3-8:

Bewertung der Haltestellenformen

Tabelle 3-9:

Typisierung der Haltestellen bzw. Teilhaltestellen hinsichtlich Haltestellenausführung und Lage der Warteflächen im Straßenquerschnitt... 33

Tabelle 3-10: $\quad$ Erhobene Merkmalskategorien und Differenzierungsmerkmale zu den untersuchten Haltestellen in den Fallbeispielen . 33

Tabelle 3-11: $\quad$ Rangfolge der Querschnittstypen mit Straßenbahn nach Unfallkostenraten 37

Tabelle 3-12: Rangfolge der Querschnittstypen ohne Straßenbahn nach Unfallkostenraten ... 38

Tabelle 3-13:

Differenzierung der Bahnkörper nach den RASt .43

Tabelle 3-14: Regellösungen bei Radverkehr auf Fahrbahnen mit Straßenbahn... 47

Tabelle 3-15: Sonderlösungen bei Radverkehr auf Fahrbahnen mit Straßenbahn.

Tabelle 3-16:

Kombinationen von Radverkehrsführung und Haltestellenform bei Straßenbahnhaltestellen in Seitenlage.

Tabelle 3-17: Bahnkörper und Bauformen 52

Tabelle 4-18:

zusammengefasste Eingangsgrößen des Verkehrsmittelvergleichs. 58

Tabelle 4-19:

Kostensätze als Grundlage zur Ermittlung der absoluten Unfallkosten .58

Tabelle 5-20:

Städte der Netzanalyse mit Übersichtskarten der georeferenzierten Unfälle 62

Tabelle 6-21:

Vergleich der unfallstatistischen Erhebungen des Bundesamtes für Statistik für Straßenbahnen 69

Tabelle 6-22: Übersicht der vorliegenden polizeilichen Unfalldaten .

Tabelle 6-23: Übersicht der vorliegenden Unfalldaten nach Unfallfolge und Art der Verkehrsbeteiligung....

Tabelle 6-24: Dateieigenschaften in Abhängigkeit des Datenformates .....

Tabelle 6-25: Datenumfang, Datenformate und Dateninhalte der erhaltenen Unfalldaten ....... 74

Tabelle 6-26: Differenzierungsmerkmale der Unfälle bei der Makro- und Mikroanalyse........... 77

Tabelle 7-27: Streckenauswahl der mikroskopischen Analyse 
Tabelle 7-28: Knotenpunktauswahl der mikroskopischen Analyse................................... 95

Tabelle 7-29: $\quad$ untersuchte Unfallstellen der mikroskopischen Analyse................................ 97

Tabelle 7-30: $\quad$ ausgewählte, wiederkehrende Defizite in Bildern ....................................... 100

Tabelle 7-31: $\quad$ Defizite nach Art der Verkehrsanlage und Art der Verkehrsteilnahme,

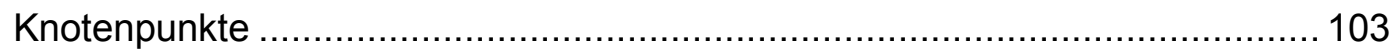

Tabelle 7-32: Defizite nach Art der Verkehrsanlage und Art der Verkehrsteilnahme,

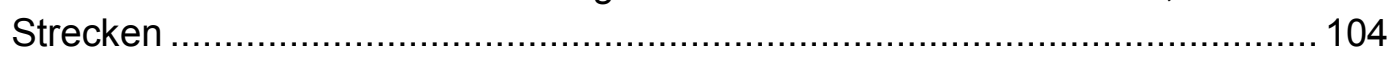




\section{Abkürzungsverzeichnis}

a

AEG

Anz.

AP

BASt

BGI

BOStrab

BStatG

DDR

DTV

EAÖ

EBO

ERA

ESAS

ESG

$f$

ff

Fzg.-km

GIS

Gt

Hausnr.

HBS

H VÖ

i.A.

i.d.R.

IV

k.A.

Kat.

$\mathrm{Kfz}$

Krad

KSV

KSS

KSU

LSA

LV

MIV

ÖPNV

ÖPSV

ÖV

PBefG

P.-km

PKW

RASt

R-FGÜ

RiLSA
Jahr (annum)

Allgemeines Eisenbahngesetz

Anzahl

Arbeitspakete

Bundesanstalt für Straßenwesen

Berufsgenossenschaftliche Information

Verordnung über den Bau und Betrieb der Straßenbahnen

Bundesstatistikgesetz

Deutsche Demokratische Republik

Durchschnittlich tägliche Verkehrsstärke

Empfehlungen für Anlagen des öffentlichen Personenverkehrs

Eisenbahn-Bau- und Betriebsordnung

Empfehlungen für Radverkehrsanlagen

Empfehlungen für das Sicherheitsaudits von Straßen

Empfehlungen zur Straßenraumgestaltung

folgende Seite

folgende Seiten

Fahrzeugkilometer

Geoinformationssystem

Getötete(r)

Hausnummer

Handbuch für die Bemessung von Straßenverkehrsanlagen

Hinweise für den Entwurf von Verknüpfungsanlagen des öffentlichen

Personennahverkehrs

Im Allgemeinen

In der Regel

Individualverkehr

keine Angaben

Unfallkategorie

Kraftfahrzeug(e)

Kraftrad

Kostensatz Verunglückte

Kostensatz anteiliger Sachschäden

Kostensatz Unfall

Lichtsignalanlage

Leichtverletzte( $r$ )

Motorisierter Individualverkehr

Öffentlicher Personennahverkehr

Öffentlicher Straßenpersonenverkehr

Öffentlicher Verkehr

Personenbeförderungsgesetz

Personenkilometer

Personenkraftwagen

Richtlinie für die Anlage von Stadtstraßen

Richtlinien für die Anlage und Ausstattung von Fußgängerüberwegen

Richtlinien für Lichtsignalanlagen 
Str.

StVG

StVO

SV

UD

UKD

UKR

$U(P)$

UR

$\mathrm{V}$

VBA

VBG

VDV

VerkStatG

vgl.

VUA

VwV-StVO
Straße

Straßenverkehrsgesetz

Straßenverkehrsordnung

Schwerverletzte $(r)$

Unfalldichte

Unfallkostendichte

Unfallkostenrate

Unfall/ Unfälle mit Personenschaden

Unfallrate

Verletzter

Visual Basic for Applications

Verwaltungs-Berufsgenossenschaft

Verband deutscher Verkehrsunternehmer

Verkehrsstatistikgesetz

Vergleiche!

Verkehrsunfallanzeige

Verwaltungsvorschrift der Straßenverkehrsordnung 
Auf dem innerörtlichen Straßennetz deutscher Städte kann seit Jahren insgesamt eine rückläufige Tendenz der Unfallzahlen, insbesondere der Getöteten und Schwerverletzten festgestellt werden. Erstmalig sank im Jahr 2013 die Zahl der Getöteten mit 977 Personen Innerorts auf unter 1.000 Getötete pro Jahr ${ }^{1}$. Der insgesamt im innerörtlichen Straßenverkehr positiv zu bewertende Trend der Entwicklung des Unfallgeschehens kann für Unfälle in Zusammenhang mit Straßenbahnen nicht verzeichnet werden².

Zwar ist die absolute Zahl der Getöteten und Schwerverletzten bei Unfällen mit Straßenbahnen bezogen auf den Gesamtverkehr vergleichsweise gering; wird dem jedoch die Fahrleistung der Modi gegenübergestellt, so muss dem Straßenbahnverkehr relativ betrachtet eine hohe Zahl von schweren Unfällen bescheinigt werden.

Dass es bei Unfällen im Zusammenhang mit Straßenbahnen typische Unfallkonstellationen und strukturelle Gemeinsamkeiten gibt, ist bereits insbesondere aus Untersuchungen an Haltestellen bekannt. Konfliktbetrachtungen und Auswertung von Unfällen kleinerer Kollektive ermöglichen es, erste Tendenzen für (un)sichere Straßenbahninfrastrukturen abzuleiten. Mit dem vorliegenden Forschungsvorhaben sollen auf Basis umfassender Unfalldaten belastbare Erkenntnisse zur Verkehrsinfrastruktur bei Unfällen mit Straßenbahnen gewonnen werden und Aussagen darüber getroffen werden, ob und durch welche Maßnahmen Straßenbahnunfälle in ihrer Anzahl oder Schwere reduziert werden können.

Ziel der Untersuchung im Rahmen des Forschungsvorhabens ist die Beantwortung folgender Forschungsfragen:

- Welche Erfahrungen bestehen zum Unfallgeschehen mit Straßenbahnen und zur Verbesserung der Verkehrssicherheit?

- Wie sicher ist der Straßenbahnbetrieb in Deutschland?

- Welche Unfalltypen, Unfallursachen und unfallbegünstigenden Umstände können festgestellt werden?

- Gibt es strukturelle Gemeinsamkeiten insbesondere bei Unfällen mit schwerem Personenschaden?

- Welche Maßnahmen können zu einer Reduktion der Unfallzahlen beitragen? 


\section{$2 \quad$ Methodik}

\subsection{Abgrenzung des Untersuchungsgegenstandes}

Straßenbahnen sind nach dem Personenbeförderungsgesetz (PBefG)

\section{(1) „, [...] Schienenbahnen, die}

1. den Verkehrsraum öffentlicher Straßen benutzen und sich mit ihren baulichen und betrieblichen Einrichtungen sowie in ihrer Betriebsweise der Eigenart des Straßenverkehrs anpassen oder

2. einen besonderen Bahnkörper haben und in der Betriebsweise den unter Nummer 1 bezeichneten Bahnen gleichen oder ähneln und ausschließlich oder überwiegend der Beförderung von Personen im Orts- oder Nachbarschaftsbereich dienen.

(2) Als Straßenbahnen gelten auch Bahnen, die als Hoch- und Untergrundbahnen, Schwebebahnen oder ähnliche Bahnen besonderer Bauart angelegt sind oder angelegt werden, ausschließlich oder überwiegend der Beförderung von Personen im Orts- oder Nachbarschaftsbereich dienen und nicht Bergbahnen oder Seilbahnen sind."3

Im Rahmen des vorliegenden Forschungsprojektes werden, basierend auf der in Kapitel 2.2 dargelegten Untersuchungsmethodik, ausschließlich ebenerdige Bahnen nach §4 Abs. 1 PBefG in der Untersuchung berücksichtigt. Eine Einbeziehung von Bahnen besonderer Bauart nach $\S 4$ Abs. 2 PBefG oder Bahnen die nach Eisenbahn-Bau- und Betriebsordnung (EBO) ${ }^{4}$ betrieben werden, erfolgt in den nachstehenden Betrachtungen nicht.

Darüber hinaus wird im Weiteren basierend auf der Verordnung über den Bau und Betrieb der Straßenbahnen (Straßenbahn-Bau- und Betriebsordnung, BOStrab ${ }^{5}$ ) $\$ 1$ die Terminologie „Straßenbahn“ auch für die gelegentlich genutzte Begrifflichkeit „Stadtbahn“ verwendet. Grundsätzlich verfügt ein Stadtbahnsystem zumeist zwar über einen höheren Komfort sowie eine bessere technische Ausstattung der Fahrzeuge, kann jedoch hier bezugnehmend auf der - dem Projekt zu Grunde liegenden - statistischen Unfalldatenbasis, den im Sinne der BOStrab betriebenen Straßenbahnen zugeordnet werden.

\section{2 methodisches Vorgehen}

Zur Bearbeitung des Forschungsvorhabens wurden im Wesentlichen zwei methodische Ansätze kombiniert:

In einem ersten Schritt erfolgte für 58 deutsche Städte mit Straßenbahnbetrieb mit den dafür vorliegenden Unfalldaten eine statistische Auswertung des Unfallgeschehens (makroskopische Analyse). Es wurden ausschließlich Unfälle mit Personenschaden unter Beteiligung von Straßenbahnen betrachtet. Dazu wurden von den betreffenden, zuständigen Innenministerien, Landespolizeidirektionen bzw. Polizeidienststellen der Länder die Unfalldaten der Jahre 2009, 2010 und 2011 angefordert. Ergänzt wurden diese Unfalldaten um straßenräumliche typisierte 
Merkmale, sodass die statistische Auswertung auf diese Typisierungsmerkmale ausgeweitet werden konnte.

Der zweite Schritt des Forschungsvorhabens bestand aus einer vertiefenden Untersuchung von Knotenpunkten und Streckenabschnitten mit auffälligem Unfallgeschehen (mikroskopische Analyse). Ziel dabei war es, Sicherheitsdefizite zu erfassen, die auf eine unsichere Gestaltung der Verkehrsanlagen zurückzuführen sind.

Ergänzt wurden diese beiden methodischen Ansätze um weitere grundlegende aufeinander aufbauende Arbeitspakete (AP).

\section{AP 1 Auswertung Fachliteratur}

Bestehende Erkenntnisse über die Sicherheit für Bau und Betrieb der Straßenbahninfrastruktur an Knotenpunkten, Strecken und Haltestellen werden durch eine eingehende Analyse bestehender Fachliteratur aufgearbeitet und zusammengefasst. Darüber hinaus werden sicherheitsrelevante Vorgaben der nationalen Regelwerke und gesetzliche Verordnungen für Bau und Betrieb der Straßenbahnverkehrsanlagen dargestellt.

\section{AP 2 Datenakquisition}

In Deutschland existieren aktuell 60 Kommunen, in denen Straßenbahnen einen integrativen Bestandteil des bestehenden Angebots des Öffentlichen Personennahverkehrs (ÖPNV) bilden. Um den Anspruch einer detaillierten Unfalluntersuchung gerecht werden zu können, wurden mit allen betreffenden Polizeidienststellen und Innenministerien der Länder Kontakt aufgenommen, um die entsprechenden statistischen Grundlagen zu erhalten. Erfragt wurden die Unfalldaten der polizeilichen Verkehrsunfallanzeigen für Unfälle mit Personenschaden unter Beteiligung von Straßenbahnen der Jahre 2009 bis 2011.

Für eine spätere Bewertung der Unfälle hinsichtlich der Straßenbahnverkehrsstärken an den jeweiligen Unfallstellen wurden je nach Verfügbarkeit Fahrpläne innerhalb der Unfalljahre oder aktuelle Fahrpläne aus dem Angebot der Verkehrsverbünde und Verkehrsbetriebe aus dem Internet heruntergeladen und ausgewertet.

Es werden ausschließlich Strecken, die nach der Verordnung über den Bau und Betrieb der Straßenbahnen (BOStrab) betrieben werden, in die Forschungsarbeit eingebunden.

\section{AP 3 Typisierung von Straßenbahnverkehrsinfrastruktur}

Zur Einordnung bzw. Kategorisierung zusätzlicher straßenräumlicher Merkmale sollen die betrachteten Knotenpunkte, Streckenabschnitte und Haltestellen typisiert werden, um mögliche Gleichartigkeiten der Unfälle in den Örtlichkeiten und der Unfallhergänge nachvollziehbar herausarbeiten zu können. Dabei wurden sowohl Differenzierungen der betrachteten Bahnkörper der freien Strecke, als auch eine differenzierte Typisierung zur Analyse des Unfallgeschehens an Knotenpunkten und Haltestellen erarbeitet. Die Aufnahme der straßenräumlichen Merkmale erfolgte im Wesentlichen auf Basis von Luftbildern und Befahrungsbildern der Kartendienste Google maps ${ }^{6}$ und Google earth ${ }^{7}$ und Luftbildern des Kartendienstes Bing Maps ${ }^{8}$. 


\section{AP 4 Analyse des Unfallgeschehens}

Zentrales Ziel dieser Studie ist die Ableitung des Zusammenhangs zwischen der Verkehrsunsicherheit durch Straßenbahnverkehrsunfälle und strukturellen bzw. infrastrukturellen Gemeinsamkeiten der betrachteten Unfälle. Dazu wurden die in AP 2 erhaltenen unfallspezifischen Daten über einen Zeitraum von drei Jahren aufbereitet und ausgewertet sowie den in AP 3 ermittelten straßenräumlichen Typisierungen zugeordnet. Durch Auswertung der Unfalldaten auf Grundlage eines umfangreichen Datenkollektivs sollen infrastrukturell begünstigende Unfallumstände herausgearbeitet werden. Zum einen erfolgte die Auswertung unfallbezogen. Das bedeutet, dass die Verteilung der einzelnen Unfall- und Typisierungsmerkmale über das Gesamtunfallgeschehen der vorliegenden Straßenbahnverkehrsunfälle betrachtet wurde. Darüber hinaus wurde neben der unfallbezogenen Betrachtung eine netzbezogene Betrachtung für fünf ausgewählte Städte durchgeführt. Damit wurde die längenbezogene Verteilung der beiden Merkmale Querschnittstyp und RASt-Entwurfssituation ermittelt und dem Unfallgeschehen gegenübergestellt. Aussagen über das Verhältnis zwischen der Häufigkeit der Merkmale und der Häufigkeit (und Schwere) der Unfälle wurden damit möglich. Zudem wurden längenbezogene Unfallkenngrößen (Unfalldichte, Unfallrate, Unfallkostendichte, Unfallkostenrate) für beide Merkmalsausprägungen ermittelt.

\section{AP 5 Detailanalyse auffälliger Unfallstellen}

Die aus AP 4 gewonnen Erkenntnisse durch Auswertung der Unfallkollektive und Unfallausprägungen bilden die Grundlage für eine vertiefende Untersuchung ausgewählter Streckenabschnitte und Knotenpunkte. Insgesamt werden 21 unfallauffällige Knotenpunkte und 11 Streckenabschnitte, im Rahmen von Ortsbegehungen, untersucht und bewertet, um bestehende Sicherheitsdefizite der Verkehrsanlagen in Analogie zu den ESAS $2002^{\circ}$ aufzunehmen.

Ziel war, bestehende Sicherheitsdefizite und unfallbegünstigende Umstände der Straßeninfrastruktur zu erfassen und mehrfach auftretende oder ähnliche Sicherheitsdefizite zu erkennen und zu beschreiben. Bestreben ist es, damit die Übertragbarkeit der örtlichen Randbedingungen auf andere Unfallsituationen herzustellen und objektive Maßnahmen (AP 7) ableiten zu können.

\section{AP 6 Auswertung, Bewertung der empirischen Daten}

Es erfolgte die Zusammenführung und Bewertung aller der aus den AP 1 bis AP 5 gewonnenen Erkenntnisse.

\section{AP 7 Ableitung von Praxisempfehlungen}

Aufbauend auf den gewonnenen Erkenntnissen sollen praxisgerechte Maßnahmen und Handlungsempfehlungen zur Anwendung in Kommunen, den Infrastrukturbetreibern und zur Einarbeitung und Konkretisierung von Regelwerken und Hinweispapieren abgeleitet werden.

\section{AP 8 Entwicklung eines Bewertungsverfahrens}

Zur Bewertung einer sicheren Infrastruktur stehen seit dem Jahr 2002 die Empfehlungen für das Sicherheitsaudit von Straßen (ESAS 2002) für Um-, Aus- und Neubau von Straßenverkehrsanlagen zur Verfügung. Eine Übertragbarkeit auf Straßen des Bestandes ist

9 [ESAS 2002] - Empfehlungen für das Sicherheitsaudit von Straßen 
möglich, auch wenn ein formalisiertes Vorgehen eines sogenannten "Bestandaudits" derzeit durch die Forschungsgesellschaft für Straßen- und Verkehrswesen (FGSV) erarbeitet wird.

In Anlehnung zu den Checklisten der ESAS 2002 wurden erkannte sicherheitsrelevante

Erkenntnisse aus dem Forschungsvorhaben als zu prüfende Defizite bei Um-, Neu-, und Ausbau oder auffälligen Unfallstellen im Bestand herausgearbeitet. 


\section{$3 \quad$ Literaturanalyse}

\subsection{Grundlagen zur Gestaltung von Straßenbahninfrastruktur}

Im folgenden Kapitel soll zunächst, ein Überblick über grundlegende Erkenntnisse aus der Literatur zur Einordnung von Straßenbahninfrastruktur in den städtischen Raum, wie auch zu baulichen Anforderungen an die Netzelementelemente Bahnkörper, Knotenpunkt und Haltstelle gegeben werden.

\subsubsection{Straßenbahninfrastruktur im städtischen Raum}

Bei Neuplanung, Erweiterung oder Änderung von Straßenbahnlinien sollte die Linienwahl hinsichtlich verkehrlicher Aspekte (Leistungsfähigkeit an Knoten in Verbindung mit dem fließenden Verkehr, etc.) als auch städtebaulicher Aspekte (Straßenraumgestaltung, Intensität der Seitenraumnutzung, etc.) geprüft werden. ${ }^{10}$

Da der Streckenführung von Straßenbahnen meist unterschiedliche Stadtstrukturen zu Grunde liegen, muss die individuelle Gestaltung des Straßenraums unter der vorherrschenden städtebaulichen und verkehrlichen Situation aller Streckenabschnitte vorgenommen werden. „Die Straßenraumbreite ist im Hinblick auf die Machbarkeit ein wesentliches städtebauliches Kriterium für die Streckenbewertung. “11 Dabei sind bei der Führung von Straßenbahnen im städtischen Raum, die bestehenden Raumansprüche (Fußgänger, Radfahrer, etc.) sowie die umliegenden Nutzungen der Randbebauung zu beachten. In Tabelle 3-1sind die sich aus diesen Ansprüchen ergebenden Mindestanforderungen für die Seitenraumbreite (zwischen Hauskante und Fahrbahn bzw. Fahrfläche der Stadtbahn) zusammengefasst.

Tabelle 3-1: Mindestanforderungen für Seitenraumbreiten

Quelle: [M schienengebundener ÖV], S. 9

\begin{tabular}{|c|c|}
\hline Breite & Raumnutzung im Seitenbereich von Straßen... \\
\hline $2 \mathrm{~m}$ & $\begin{array}{l}\text {.. ohne nennenswerte Randnutzung } \\
\text { (kein Wirtschaftsverkehr) }\end{array}$ \\
\hline $3 m$ & $\begin{array}{l}\text {... mit mäßiger Aufenthaltsfunktion } \\
\text { (geringer Fußgängerlängsverkehr, geringer Wirtschaftsverkehr) }\end{array}$ \\
\hline $4 m$ & $\begin{array}{l}\text {... mit gemischter Randnutzung } \\
\text { (notwendiger Bedarf an Wirtschaftsflächen für Auslagen, Bistro-Tische u.Ä., } \\
\text { Fußgängerlängs- und querverkehr) }\end{array}$ \\
\hline $5 \mathrm{~m}$ & $\begin{array}{l}\text {... mit intensiver Aufenthaltsfunktion } \\
\text { (größerer Bedarf an Wirtschaftsflächen für Auslagen, intensiver } \\
\text { Fußgängerverkehr) }\end{array}$ \\
\hline $6 m$ & $\begin{array}{l}\text {.. mit intensiver Aufenthaltsfunktion } \\
\text { (großer Bedarf an Wirtschaftsflächen, intensiver Fußgängerverkehr, erlaubt } \\
\text { Warteflächen für Haltestellen) }\end{array}$ \\
\hline
\end{tabular}

10 [M schienengebundener ÖV 2003], S. 8

11 [M schienengebundener ÖV 2003], S. 8 
Aus den Nutzungsansprüchen und damit den genannten Mindestanforderungen für die Seitenraumbreite können Mindestbreiten für die Integration von Straßenbahninfrastruktur in den Straßenraum abgeleitet werden:

$\begin{array}{ll}\text { Tabelle 3-2: } & \begin{array}{l}\text { Mindestraumbreiten zur Integration von Straßenbahninfrastruktur unter Beachtung der } \\ \text { Straßenraumnutzung }\end{array} \\ \text { Quelle: } & {[\text { M schienengebundener ÖV], S. } 10}\end{array}$

\begin{tabular}{|l|l|}
\hline Breite des Straßenraums & Raumnutzung \\
\hline \multirow{3}{*}{$\mathrm{B} \geq 20 \mathrm{~m}$} & Integration von Straßenbahninfrastruktur ohne wesentliche \\
& Einschränkungen weiterer Nutzungen möglich: \\
& - Fuß \\
& - Rad \\
& - Ruhender Verkehr \\
\hline \multirow{3}{*}{$20 \mathrm{~m}<\mathrm{B} \leq 16 \mathrm{~m}$} & Integration von Straßenbahninfrastruktur mit Qualitätsverlust \\
& für weitere Nutzungen möglich: \\
& - Fuß \\
& - Rad \\
& - evtl. ruhender Verkehr \\
\hline \multirow{3}{*}{$16 \mathrm{~m}<\mathrm{B} \leq 12 \mathrm{~m}$} & Integration von Straßenbahninfrastruktur nur mit \\
& Einschränkung anderer Raumfunktionen möglich: \\
& - Fuß \\
\hline \multirow{2}{*}{$\mathrm{B}<12 \mathrm{~m}$} & Integration von Straßenbahninfrastruktur nicht oder nur auf \\
& kurzen Streckenabschnitten möglich. \\
\hline
\end{tabular}

Aus den zur Verfügung stehenden Straßenraumbreiten können unter Beachtung der dargelegten städtebaulichen Aspekte und Mindestanforderungen die folgenden, in Kapitel 3.1.2 beschriebenen, Entwurfstypen für Bahnkörper und Haltestellen abgeleitet werden.

\subsubsection{Netzelemente der Straßenbahninfrastruktur}

Die Netzelemente der Straßenbahninfrastruktur im öffentlichen Straßenraum können im Wesentlichen folgenden Bereichen zugeordnet werden:

- Bahnkörper der freien Strecke (d.h. außerhalb des Einflusses von Knotenpunkten),

- Knotenpunkte und

- Haltestellen

Haltestellen nehmen dabei einen gesonderten Stellenwert ein, da sie gegenüber Bahnköpern der freien Strecke und Knotenpunkten als dazu ergänzendes Element angesehen werden können. 


\subsubsection{Bahnkörper}

Bahnkörper können grundsätzlich nach [BOStrab 2007] ${ }^{12}$ in drei Bauarten unterschieden werden:

- straßenbündiger Bahnkörper (Einbettung der Gleise i.d.R. in die Fahrbahn)

- besonderer Bahnkörper (liegen im Verkehrsraum, sind jedoch durch ortsfeste Hindernisse vom übrigen Verkehr getrennt)

- unabhängiger Bahnkörper (durch die Bauart oder die Lage unabhängig vom übrigen Verkehr)

Verkehrende Züge auf straßenbündigen Bahnkörpern nehmen nach [BOStrab 2007] ${ }^{13}$ am Straßenverkehr teil und müssen daher die betreffenden Vorschriften nach StraßenverkehrsOrdnung (StVO) einhalten. Züge, die auf besonderen oder unabhängigen Bahnkörpern verkehren, nehmen nach [BOStrab 2007] $]^{14}$ nicht am Straßenverkehr teil.

Neben der Wahl der Bauart ist ebenfalls die Führung im Querschnitt von besonderer baulicher und verkehrlicher Bedeutung. Nach [RASt 2006] ${ }^{15}$ können unter Beachtung

- der örtlich zu Grunde liegenden Nutzungs- und Raumansprüche,

- Kraftfahrzeugverkehrsstärke und der

- Straßenraumbreite

mittels einer geeigneten Kombination von Querschnittselementen typische Entwurfssituationen abgebildet werden, die in etwa $70 \%$ bis $80 \%$ der in der Praxis auftretenden Entwurfsaufgaben abdecken. Bei der Auswahl empfohlener Straßenquerschnitte mit Straßenbahnverkehr stehen nach [RASt 2006] grundsätzlich zwei Führungsformen zur Auswahl, die weiterführend in Abhängigkeit der Entwurfssituation und der zur Verfügung stehenden Straßenraumbreite in unterschiedliche empfohlene Querschnitte unterteilt werden können:

Tabelle 3-3: $\quad$ Führung des Bahnkörpers im Straßenraum

\begin{tabular}{|l|l|}
\hline Führung des Bahnkörpers in Mittellage & Führung des Bahnkörpers in Seitenlage \\
\hline &
\end{tabular}

Die Führung als straßenunabhängiger Bahnkörper wird in den RASt 2006 keine weitere Bedeutung zugemessen, da das Regelwerk "den Entwurf und die Gestaltung von Erschließungsstraßen und [...] Hauptverkehrsstraßen"16 behandelt und sich somit Verkehrsräumen mit mehreren innerörtlichen Nutzungsansprüchen widmet.

\footnotetext{
[BOStrab 2007], §16 (4)

[BOStrab 2007], §55 (1)

[BOStrab 2007], §55 (3)

[RASt 2006], S. 33

[RASt 2006], S. 13
} 
Aus den Abbildungen der "empfohlene[n] Lösungen für typische Entwurfssituationen"17 ergeben sich folgende Querschnittsaufteilungen und den zu erkennenden Querschnittsbreiten für Straßen mit Straßenbahnverkehr:

Tabelle 3-4: $\quad$ Mindestraumbreiten zur Integration von Straßenbahninfrastruktur unter Beachtung der Straßenraumnutzung

Quelle: $\quad$ [RASt 2006], S. 50ff

\begin{tabular}{|c|c|}
\hline Querschnitt & Prinzipskizze mit Bemaßung \\
\hline Fußgängerzone & \\
\hline $\begin{array}{l}\text { Mittellage, straßenbündig, } \\
1 \text { Kfz-Fahrstreifen je Richtung }\end{array}$ & \\
\hline $\begin{array}{l}\text { Mittellage, straßenbündig, } \\
2 \text { Kfz-Fahrstreifen je Richtung }\end{array}$ & \\
\hline \multicolumn{2}{|l|}{$\begin{array}{l}\text { Mittellage, besonderer Bahnkörper, } \\
1 \mathrm{Kfz} \text {-Fahrstreifen je Richtung }\end{array}$} \\
\hline \multicolumn{2}{|l|}{$\begin{array}{l}\text { Mittellage, besonderer Bahnkörper, } \\
2 \text { Kfz-Fahrstreifen je Richtung }\end{array}$} \\
\hline \multicolumn{2}{|l|}{ Seitenlage } \\
\hline & \\
\hline
\end{tabular}

In Ergänzung dazu kann nach dem Merkblatt für die Gestaltung von Anlagen des schienengebundenen Verkehrs ${ }^{18}$ in folgende Ausbauformen unterschieden werden:

- Straßenbündige Bahnkörper mit zeitlicher Trennung,

- Straßenbündige Bahnkörper mit räumlicher Trennung,

- Besondere Bahnkörper mit geschlossenem Oberbau,

17 [RASt 2006], S. 33ff

18 [M schienengebundener ÖV 2003], S. 13 
- Besondere Bahnkörper mit begrüntem Oberbau und

- Besondere Bahnkörper mit geschottertem Oberbau.

\subsubsection{Knotenpunkte}

Im innerörtlichen Straßennetz sind überwiegend plangleiche (niveaugleiche) Kreuzungen und Einmündungen vorzufinden.

In Anlehnung an die [RASt 2006] ${ }^{19}$ sowie [WEISE, DURTH et al. 1997] ${ }^{20}$ lassen sich plangleiche Knotenpunkte in Abhängigkeit von der Gestaltung und technischen Sicherung in folgende typische Grundformen einordnen, wie sie in Zusammenhang mit Straßenbahnverkehrsanlagen vorzufinden sind:

Tabelle 3-5: Knotenpunktgrundformen in Zusammenhang mit Straßenbahnverkehrsanlagen

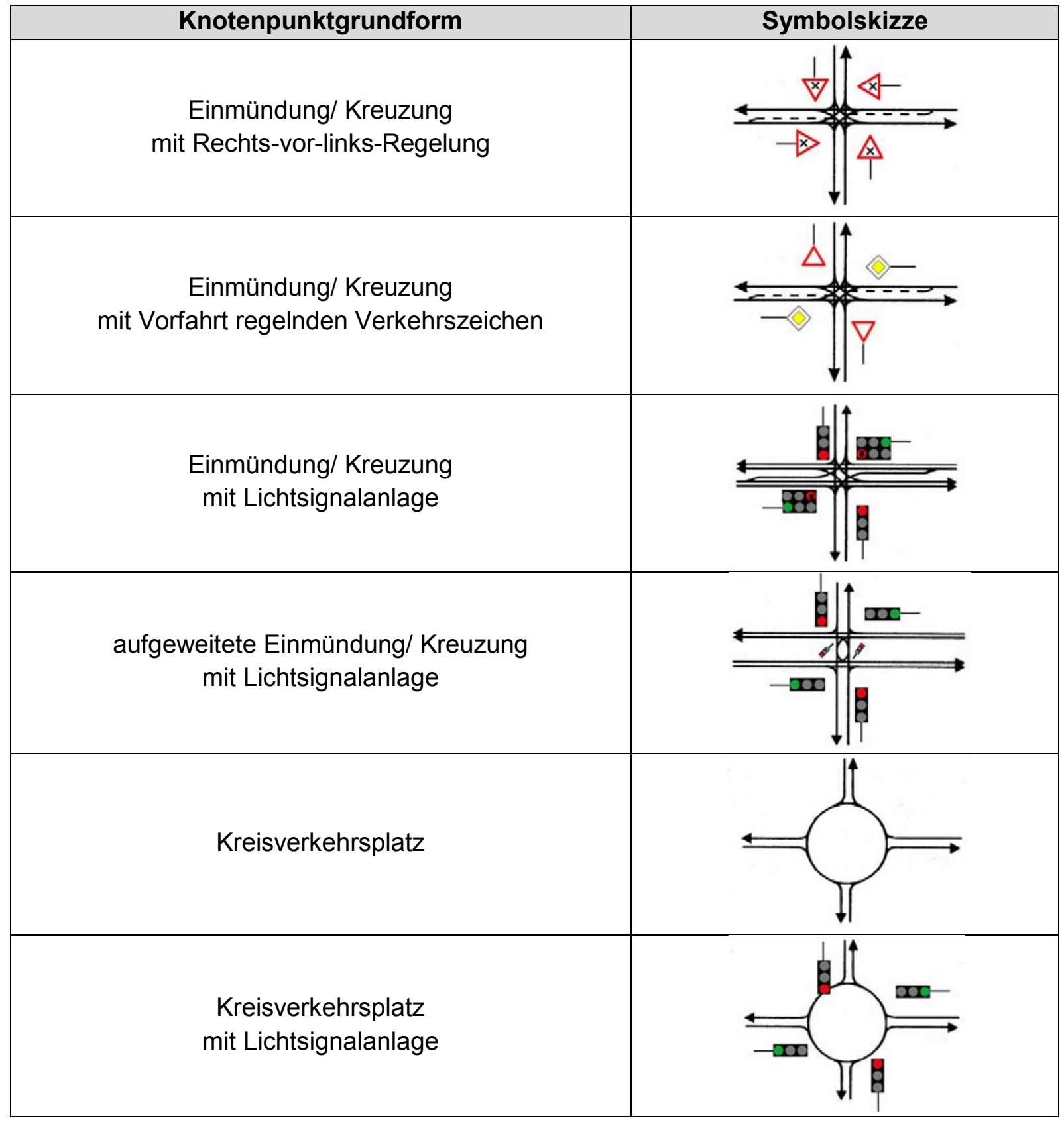

19 [RASt 2006], S. 109ff

20 [WEISE, DURTH et al. 1997], S. 259ff 


\subsubsection{Haltestellen}

Haltestellen kommt im Rahmen der Gestaltung von Strecken und Knotenpunkten eine besondere Bedeutung zu, da mit Haltestellen nach [EAÖ 2013] ${ }^{21}$ stets besondere Anforderungen im Zusammenhang stehen:

- die Anforderungen der Fahrgäste, u.a.

- Quell- und Zielnähe (Erschließungsfunktion),

- Verknüpfung zwischen den Fahrzeugen des ÖV und anderen Modi (Verbindungsfunktion)

- sichere, barrierefreie und komfortable Erreichbarkeit

- ausreichende Dimensionierung und Aufenthaltsqualität der Warteflächen

- die Anforderungen des Betreibers, u.a.

- sichere Fahrgastwechsel und kurze Fahrgastwechselzeiten

- sichere, zügige, störungsfreie Haltestellenein- und Haltestellenausfahrt der Fahrzeuge

- die Anforderungen anderer Straßennutzer, u.a.

- gute Erkennbarkeit der Haltestelle, der ÖV-Fahrzeuge und Fahrgäste

- ausreichende Dimensionierung der verbleibenden Verkehrsinfrastruktur und sichere Führung aller Verkehrsteilnehmer

Die Bedeutung der Verkehrssicherheit und einer ausreichenden Dimensionierung wird dabei deutlich. Damit stehen Haltestellen noch mehr als Strecken und Knotenpunkte ohne Haltestellen im Wechselspiel zu Flächenkonkurrenz, der Umfeldnutzung und stadtgestalterischen Anforderungen. ${ }^{22}$

Haltestellen können grundsätzlich nach ihrer Form unterschieden werden. Die Wahl der Haltestellenform und deren Dimensionierung hängt dabei vorrangig von den folgenden Faktoren $a b^{23}$ :

- fahrzeugtechnische Anforderungen (bspw.: Hoch-/ Niederflurtechnik, Zuglängen, etc.)

- straßenräumliche Anforderungen (Nutzungsansprüche aller Verkehrsteilnehmer, städtebauliche Aspekte, Zugänglichkeit, etc.)

- Fahrgastpotential

„Die wesentlichen Grundformen von Haltestellen ergeben sich durch die Zuordnung der Wartefläche für Fahrgäste zu den Halteplätzen der Nahverkehrsfahrzeuge und der Zugangsart“24 Elementar für die Typisierung ist dabei die Lage der Haltestelle in Mittel- oder Seitenlage. Befinden sich Haltestellen in Mittellage kann ebenfalls noch einmal zwischen den Möglichkeiten einer zusammenliegenden Halte- und Wartefläche oder getrennten Bereichen für Halten der Straßenbahn und Warten des Fahrgastes unterschieden werden.

Aus diesen Differenzierungen ergeben sich die in Tabelle 3-6 dargelegten Haltestellenformen.

[EAÖ 2013], S. 60

[EAÖ 2013], S. 60

3 [M schienengebundener ÖV 2013], S. 15

24 [M schienengebundener ÖV 2013], S. 15 
Tabelle 3-6: Haltestellenformen des schienengebundenen ÖV Quelle: $\quad$ nach [M schienengebundener ÖV], S. 16f

\begin{tabular}{|c|c|c|c|}
\hline Merkmal & Grundform A & Grundform B & Grundform C \\
\hline Gleislage & Seitenlage & Mittellage & Mittellage \\
\hline \multirow{13}{*}{$\begin{array}{l}\text { Wartefläche/ } \\
\text { Haltefläche }\end{array}$} & zusammenliegend & getrennt & zusammenliegend \\
\hline & 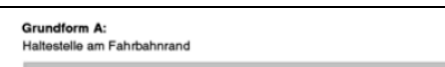 & 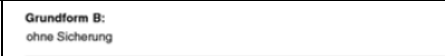 & 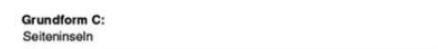 \\
\hline & 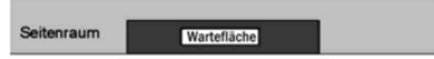 & Setenraum $\square$ & 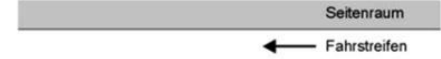 \\
\hline & 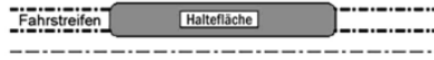 & $\begin{array}{l}\text { Fahrsteten } \\
-\cdots-\cdot-\cdot-\cdot Y\end{array}$ & \begin{tabular}{l|l} 
Waterasche & \\
\end{tabular} \\
\hline & & - & 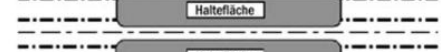 \\
\hline & 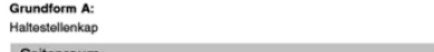 & 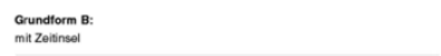 & 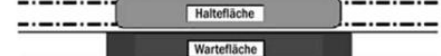 \\
\hline & $(\mathbf{P})$ Wartelicata & Setteraraum & $\longrightarrow$ Fanstreten \\
\hline & 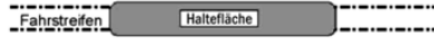 & Fahrsteten & Setterraum \\
\hline & & 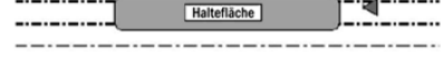 & 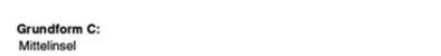 \\
\hline & & & $\begin{array}{r}\text { Setonraum } \\
\longleftarrow \text { Fanststeten }\end{array}$ \\
\hline & & & {$[\ldots$} \\
\hline & & & Wartersene \\
\hline & & & $\longrightarrow$ Saltontrum \\
\hline $\begin{array}{l}\text { Bauliche } \\
\text { Differenzierung }\end{array}$ & $\begin{array}{l}\text { Haltestelle am } \\
\text { Fahrbahnrand, } \\
\text { Haltestellenkap }\end{array}$ & - & $\begin{array}{c}\text { Haltestelle als Seiteninsel, } \\
\text { Haltestelle als Mittelinsel }\end{array}$ \\
\hline $\begin{array}{l}\text { Differenzierung } \\
\text { nach } \\
\text { technischer } \\
\text { Sicherung }\end{array}$ & - & $\begin{array}{c}\text { ohne Sicherung, } \\
\text { dynamische Haltestelle } \\
\text { (mit Zeitinsel) }\end{array}$ & - \\
\hline
\end{tabular}

\subsection{Forschungs- und Fachpublikationen}

Einen Überblick über bereits bestehende Erkenntnisse, insbesondere aus abgeschlossenen Forschungsprojekten zum Unfallgeschehen mit Straßenbahnen, soll im folgenden Kapitel gegeben werden.

Im Rahmen der Analyse bisher publizierter Forschungsergebnisse mit Bezug zur Verkehrssicherheit der Straßenbahninfrastruktur können die enthaltenen Inhalte im Wesentlichen den folgenden zwei Schwerpunkten zugeordnet werden:

- Untersuchungen zum Unfallgeschehen des ÖPNV an Haltestellen (vergleichend auch Bus und Straßenbahn)

- Untersuchungen zum Unfallgeschehen mit Straßenbahnen auf der freien Strecke.

Die Betrachtung der Verkehrssicherheit in Knotenpunktbereichen findet sich je nach Abgrenzung der vorliegenden Untersuchungen in beiden Schwerpunkten wieder.

Darüber hinaus existieren ebenfalls Untersuchungen und Analysen bzgl. der Aspekte:

- Sicherheitstechnologien im ÖPNV ("security")

- Sicherheit im Fahrdienst und Umgang betroffener Mitarbeiter des ÖPNV mit Extremerlebnissen 
- Schulungen von Fahrpersonal im ÖPNV

die jedoch den Inhalten dieses Forschungsprojektes nicht unmittelbar zugeordnet werden können und auf die daher in den folgenden Ausführungen nicht weiter eingegangen werden soll.

\subsubsection{Sicherheitsuntersuchungen an Haltestellen des ÖPNV}

\begin{tabular}{|c|c|c|c|}
\hline Titel: & \multicolumn{3}{|c|}{$\begin{array}{l}\text { Möglichkeiten zur Verbesserung der Sicherheit } \\
\text { an Haltestellen im Straßenraum }\end{array}$} \\
\hline Veröffentlicht: & \multicolumn{3}{|c|}{ 1980, erste Studie zum Thema durch BASt } \\
\hline Autor(en): & $\begin{array}{l}\text { Fiedler, Hamann, } \\
\text { Strünkmann, Linnenberg }\end{array}$ & Quellenangabe: & [FIEDLER et al. 1980] \\
\hline
\end{tabular}

Im Rahmen des Forschungsprogramms „Innerstädtische Planung als Einflussgröße auf die Verkehrssicherheit“ wurde erstmals im Jahr 1980 eine Pilotstudie bzgl. der „Möglichkeiten zur Verbesserung der Sicherheit an Haltestellen im Straßenraum" durch die Bundesanstalt für Straßenwesen (BASt) beauftragt. Ziel der Studie war es, eine Prüfung des Einflusses baulicher Faktoren der Infrastruktur wie auch Faktoren des ÖPNV Betriebs an Haltestellen durchzuführen und erste Aussagen über grundsätzliche Möglichkeiten zur Verbesserung der Verkehrssicherheit in Haltstellenbereichen treffen zu können. Im Ergebnis der Forschungsarbeit werden:

- verkehrliche,

- betriebliche,

- verkehrstechnische,

- bauliche und

- umweltbezogene

Anforderungen an die Gestaltung von Haltestellen beschrieben.

Als unfallbegünstigende Faktoren in Haltestellenbereichen wurden insbesondere folgende Konfliktsituationen aufgezeigt:

- bei Annährung bzw. Halten des ÖPNV an der Haltstelle: Konflikte zwischen motorisiertem Individualverkehr (MIV) und Fußgänger/ Radfahrer

- bei Wiedereinordnung des ÖPNV in den Verkehr: Konflikte zwischen MIV und ÖPNV-Fahrzeug

Basis der Forschungsarbeit waren insbesondere Diskussionen mit den zuständigen Stellen der Polizeibehörden wie auch ausgewählter Nahverkehrsbetriebe und durchgeführte Detailanalysen der Forschergruppe an ausgewählten Haltestellen. Eine Erhebung mit Ziel einer umfassenden empirischen Datenlage zur Unterstützung der beschriebenen Erkenntnisse wurde nicht umgesetzt. Als weiterer Forschungsbedarf werden daher sowohl verhaltensbezogene Untersuchungen der Fahrgäste im Umfeld der Haltestelle (Erreichen und Verlassen der Haltestelle bzw. der Wartefläche, Fahrgastwechsel, etc.) als auch die Führung des ÖPNV bei der Wiedereinordnung in den Verkehr nach Verlassen der Haltestelle benannt. 


\begin{tabular}{|l|l|l|l|}
\hline Titel: & \multicolumn{3}{l|}{ Fußgängersicherheit an Haltestellen } \\
\hline Veröffentlicht: & 1985, Folgestudie der BASt & \\
\hline Autor(en): & Hamann & Quellenangabe: & [HAMANN 1985] \\
\hline
\end{tabular}

Basierend auf den Ergebnissen der durch die BASt veröffentlichten Studie wurde 1985 eine weitere Untersuchung zum Thema „Fußgängersicherheit an Haltestellen“ ebenfalls durch die BASt beauftragt. Diese griff insbesondere die Erkenntnisse bzgl. des Verhaltens von Fahrgästen in Haltestellen- und in Querungsbereichen auf. Darüber hinaus wurden ebenfalls die Ergebnisse einer Studie der Freien Hansestadt Bremen bzgl. einer ÖPNV-Priorisierung für Busse an Lichtsignalanlagen (LSA) und deren Auswirkungen auf die Fußgängersicherheit mit in die weiteren Forschungen integriert.

Ziel der Arbeit war es zum einen Gefahrenstellen aber auch das Verhalten von Fußgängern in Haltestellenbereichen - auch in Verbindung mit Beschleunigungsmaßnahmen des ÖV an LSA aufzuzeigen. Dazu wurden in einem ersten Schritt relevante Informationen zum Unfallgeschehen mit Fußgängern an Haltestellen zusammengetragen ${ }^{25}$ :

Es werden deutlich mehr Unfälle an Haltestellen als auf der freien Strecke verzeichnet. Straßen mit Straßenbahnen in Mittellage gehören zu den Straßen mit der höchsten Unfalldichte.

Folgende Erkenntnisse zu den Verhaltensweisen von Fußgängern und Fahrgästen wurden benannt ${ }^{26}$ :

- Das Kriterium „kürzester Weg“ ist ausschlaggebend für die Wahl des Weges von/ zur Haltestelle von Fahrgästen.

- Es kann ein Querungsverhalten in Abhängigkeit des Alters festgestellt werden.

- Besondere Verhaltensweisen des MIV (Geschwindigkeitsreduktion, höhere Aufmerksamkeit für das Verkehrsgeschehen, etc.) entstehen bei Fußgängergruppen; dieses Phänomen tritt jedoch auch außerhalb von Haltestellenbereichen auf.

Aufbauend auf diesen Erkenntnissen wurden mittels eines entwickelten Beobachtungsbogens vorOrt-Begehungen durchgeführt, um Verhaltensmuster von Fußgängern aufzeigen zu können. Dabei wurden fünf LSA-Bereiche, in zwei Untersuchungsschritten - sowohl ohne als auch mit ÖPNV Beschleunigung - in Haltestellennähe untersucht. Folgende Erkenntnisse können aus diesen Beobachtungen zusammengefasst werden ${ }^{27}$ :

"Laufeinsteiger" bilden den kleinsten Anteil an Fahrgästen, sind jedoch besonders gefährdet, da eine erhöhte Unaufmerksamkeit für das übrige Verkehrsgeschehen zu verzeichnen ist.

LSA geregelte Querungshilfen werden bei fehlender Koordinierung zu den ÖV-Halten durch Einsteiger, insbesondere „Laufeinsteiger“, wenig genutzt bzw. akzeptiert.

Einrichtungen von ÖV Beschleunigungsmaßnahmen haben die Querungssituation für Aussteiger verbessert, aber für Einsteiger verschlechtert.

Insgesamt zeigt sich, dass die Gruppe der Laufeinsteiger als gefährdetste Gruppe angesehen werden kann. Die Einrichtung von ÖV-Beschleunigungsmaßnahmen in Haltstellenbereichen verstärkt diesen Umstand zusätzlich, da die LSA-geregelten Querungshilfen erst für die 
aussteigenden Fahrgäste freigegeben werden. Um diesem Zustand entgegen zu wirken, wird im Rahmen der Signalisierung der Nutzen von Zeitinseln aufgeführt als auch darüber hinaus weitere Ansprüche an die Erkennbarkeit von Haltestellen gestellt (zusätzliche Markierungen und Beschilderungen aber auch eine verkehrsgünstige Lage).

\begin{tabular}{|c|c|c|c|}
\hline Titel: & \multicolumn{3}{|c|}{$\begin{array}{l}\text { Verkehrsgerechte Lage von Haltestellen im } \\
\text { Straßenraum unter dem Aspekt der Verkehrssicherheit }\end{array}$} \\
\hline Veröffentlicht: & \multicolumn{3}{|c|}{ 1988, Dissertation } \\
\hline Autor(en): & Linnenberg & Quellenangabe: & [LINNENBERG 1988] \\
\hline
\end{tabular}

Aufbauend auf den beiden zuvor genannten Forschungsprojekten erfolgte an der Bergischen Universität Wuppertal die Erstellung einer Dissertation zum Thema „Verkehrsgerechte Lage von Haltestellen im Straßenraum unter dem Aspekt der Verkehrssicherheit“. Im Rahmen dieser Arbeit wurden Gefährdungspotentiale nach potentiellen „Konfrontationen“:

- Kfz und Fahrgast

- Kfz und ÖV-Fahrzeug

- Kfz und Rad

- ÖV-Fahrzeug und Fahrgast

- ÖV-Fahrzeug und Rad

- Fahrgast und Rad

für die vier Haltestellenformen:

- Haltestelle in Mittellage mit Haltestelleninsel

- Haltestelle in Mittellage ohne Haltestelleninsel

- Haltestelle in Seitenlage mit Haltestellenbucht

- Haltestelle in Seitenlage ohne Haltestellenbucht

untersucht.

Basierend auf einer Diskussion von Merkmalen und Einsatzbereichen verschiedener Untersuchungsmethoden zur Beschreibung des Verkehrsgeschehens an Haltestellen wurde die „Verkehrssituationsuntersuchung“ gewählt. Kern dieser Untersuchungsmethode bildet die Erfassung von Gehlinien einzelner Fußgänger, wie auch Vermerke über beobachtete Gefährdungspotentiale auf einer Lageskizze des Haltestellenbereichs. Im Rahmen der Dissertation wurde das Verkehrsgeschehen an 94 Haltestellen erfasst.

In Tabelle 3-7 wird eine Zusammenfassung über die Gefährdungspotentiale zwischen den Konfrontationsgruppen an den untersuchten Haltestellen gegeben. 
Tabelle 3-7: Gefährdungspotentiale nach Haltestellenformen

Quelle: nach [LINNENBERG 1988], S. 26ff

\begin{tabular}{|c|c|c|c|c|}
\hline $\begin{array}{l}\text { Gefährdungs } \\
\text { potentiale }\end{array}$ & $\begin{array}{l}\text { Haltestelle in } \\
\text { Mittellage mit } \\
\text { Haltestelleninsel }\end{array}$ & $\begin{array}{l}\text { Haltestelle in } \\
\text { Mittellage ohne } \\
\text { Haltestelleninsel }\end{array}$ & $\begin{array}{l}\text { Haltestelle in } \\
\text { Seitenlage mit } \\
\text { Haltestellenbucht }\end{array}$ & $\begin{array}{l}\text { Haltestelle in } \\
\text { Seitenlage ohne } \\
\text { Haltestellenbucht }\end{array}$ \\
\hline $\begin{array}{l}\text { Einsatz- } \\
\text { bereiche }\end{array}$ & $\begin{array}{l}\text { Bus } \\
\text { Straßenbahn } \\
\text { (besonderer } \\
\text { Bahnkörper) }\end{array}$ & $\begin{array}{l}\text { Straßenbahn } \\
\text { (straßenbündiger } \\
\text { Bahnkörper) }\end{array}$ & $\begin{array}{l}\text { Bus } \\
\text { Straßenbahn } \\
\text { (besonderer } \\
\text { Bahnkörper) }\end{array}$ & $\begin{array}{l}\text { Bus } \\
\text { Straßenbahn } \\
\text { (straßenbündiger } \\
\text { Bahnkörper) }\end{array}$ \\
\hline $\begin{array}{l}\text { zwischen Kfz } \\
\text { und Fahrgast }\end{array}$ & $\begin{array}{l}\text { Queren der } \\
\text { Fahrstreifen }\end{array}$ & $\begin{array}{l}\text { Nutzung der } \\
\text { Fahrbahn durch ein- } \\
\text { und aussteigende } \\
\text { Fahrgäste, } \\
\text { Hinterlaufen } \\
\text { haltender ÖV- } \\
\text { Fahrzeuge }\end{array}$ & $\begin{array}{l}\text { Doppel-Nutzung der } \\
\text { Haltestellenbucht }\end{array}$ & $\begin{array}{l}\text { Queren der } \\
\text { Fahrbahn } \\
\text { Bemessung der } \\
\text { Warteflächengröße }\end{array}$ \\
\hline $\begin{array}{l}\text { zwischen Kfz } \\
\text { und ÖV- } \\
\text { Fahrzeug }\end{array}$ & $\begin{array}{l}\text { Ein- und Ausfädeln } \\
\text { des ÖV in/ aus dem } \\
\text { Verkehrsstrom des } \\
\text { Individualverkehrs }\end{array}$ & $\begin{array}{l}\text { Fahrstreifenwechsel } \\
\text { des Kfz-Verkehrs }\end{array}$ & $\begin{array}{l}\text { Wiedereinordnung } \\
\text { des ÖV in den } \\
\text { fließenden Verkehr }\end{array}$ & $\begin{array}{l}\text { Fahrstreifenwechsel } \\
\text { des Kfz-Verkehrs }\end{array}$ \\
\hline $\begin{array}{l}\text { zwischen Kfz } \\
\text { und Rad }\end{array}$ & $\mathrm{Cr} \mathrm{CH}_{\mathrm{CH}}$ & - & 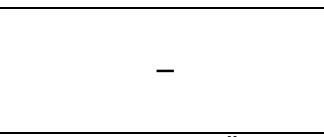 & $\begin{array}{l}\text { Vorbeifahrt Rad an } \\
\text { haltendem ÖV/ Kfz- } \\
\text { Verkehr }\end{array}$ \\
\hline $\begin{array}{l}\text { zwischen ÖV- } \\
\text { Fahrzeug und } \\
\text { Fahrgast }\end{array}$ & $\begin{array}{l}\text { Hinterlaufen } \\
\text { haltender ÖV- } \\
\text { Fahrzeuge }\end{array}$ & $\begin{array}{l}\text { Hinterlaufen } \\
\text { haltender ÖV- } \\
\text { Fahrzeuge }\end{array}$ & $\begin{array}{l}\text { Einfahren des ÖV in } \\
\text { die Bucht } \\
\text { Warten von } \\
\text { Passanten in, im } \\
\text { Raum der Haltestelle } \\
\text { gelagerten, } \\
\text { Fußgängerfurt }\end{array}$ & $\begin{array}{l}\text { Hinterlaufen } \\
\text { haltender ÖV- } \\
\text { Fahrzeuge }\end{array}$ \\
\hline $\begin{array}{l}\text { zwischen ÖV- } \\
\text { Fahrzeug und } \\
\text { Rad }\end{array}$ & - & - & $\begin{array}{l}\text { Kreuzen der } \\
\text { Fahrlinien von ÖV } \\
\text { und Rad }\end{array}$ & $\begin{array}{l}\text { Vorbeifahrt Rad an } \\
\text { haltendem ÖV/ Kfz- } \\
\text { Verkehr }\end{array}$ \\
\hline $\begin{array}{l}\text { zwischen } \\
\text { Fahrgast und } \\
\text { Rad }\end{array}$ & - & $\begin{array}{l}\text { Durchfahren der } \\
\text { Wartefläche beim } \\
\text { Fahrgastwechsel }\end{array}$ & $\begin{array}{l}\text { Doppelnutzung } \\
\text { Radweg und } \\
\text { Wartefläche }\end{array}$ & $\begin{array}{l}\text { Doppelnutzung } \\
\text { Radweg und } \\
\text { Wartefläche }\end{array}$ \\
\hline $\begin{array}{l}\text { Haltestellen- } \\
\text { übergreifend }\end{array}$ & \multicolumn{4}{|c|}{$\begin{array}{l}\text { Queren der Fahrbahn durch Fahrgäste, } \\
\text { Erkennbarkeit von Haltestellen, } \\
\text { Sichtfeld von ÖV-Führern, } \\
\text { Einfluss der Warteflächengröße auf das Verhalten von Fahrgästen und Passanten. }\end{array}$} \\
\hline
\end{tabular}

Wiederkehrende Gefährdungspotentiale bei nahezu allen betrachteten Haltestellen konnten im:

- Queren von Fahrstreifen durch Fahrgäste

- Hinterlaufen haltender ÖV-Fahrzeuge durch Fahrgäste und

- Überholen des haltenden ÖV durch den Kfz-Verkehr

festgestellt werden.

Basierend auf den erkannten Gefährdungspotentialen wurden entsprechend der benannten einzelnen Verkehrssituationen direkte Maßnahmen zur Erhöhung der Verkehrssicherheit abgeleitet. Diese liegen insbesondere in:

- der Verbesserung der Erkennbarkeit von Haltestellen wie auch der Markierung und Beschilderung in Haltestellenbereichen

- der Verbesserung der Verkehrsführung in Haltestellenbereichen (LSA-Sicherung, technische Ausfahrtregelungen für den ÖV, etc.) 
- der fahrzeugtechnischen Ausstattung des ÖV (optische Tür-Signale an der Fahrzeugseite, etc.)

- bauliche Maßnahmen (Haltestellen-Kaps, Vergrößerung der Warteflächen, etc.)

- Geschwindigkeitsdämpfende Maßnahmen,

- Bewusstseinsbildung aller Verkehrsteilnehmer.

Darüber hinaus wurde ebenfalls untersucht, ob bei Schülerverkehren zusätzliche Aspekte im Rahmen der Verkehrssicherheit an Haltestellen beachtet werden müssen bzw. Besonderheiten bzgl. der Gefährdungspotentiale erkannt werden können. Dabei konnte festgestellt werden, dass nur eine unzureichende Verringerung der Geschwindigkeit der Kfz an Haltestellen mit Schülergruppen besteht. Lediglich eine weiter abgesetzte Fahrlinie vom Haltestellenbord durch die Kfz wurde beobachtet ${ }^{28}$.

Als Maßnahmen wurde die Bewusstseinsbildung von Kfz-Führern, Maßnahmen zur Verbesserung der Erkennbarkeit von Haltestellen und die Erhöhung polizeilicher Geschwindigkeitskontrollen in Haltestellen-Bereichen vorgeschlagen.

\begin{tabular}{|l|l|l|l|}
\hline Titel: & \multicolumn{3}{|l|}{ ÖPNV Haltestellen mit Kap } \\
\hline Veröffentlicht: & 1990, Diplomarbeit & \multicolumn{2}{l|}{} \\
\hline Autor(en): & Dittemer & Quellenangabe: & [DITTEMER 1990] \\
\hline
\end{tabular}

Im Jahr 1990 stand die Untersuchung des Verkehrsablaufs, wie auch der Verkehrssicherheit an „ÖPNV Haltestellen mit Kap“ im Rahmen einer Diplomarbeit an der Universität Kaiserslautern im Fokus einer Verkehrssicherheitsbetrachtung. In einem ersten Schritt wurden dabei die Einsatzbereiche von Haltestellen-Kaps an Hand der Kriterien ${ }^{29}$ :

- Funktion der Straße

- Querschnittsbelastung IV

- Querschnittsbelastung ÖV

- Fahrgastaufkommen

aufgezeigt.

Basis bildete eine an 140 Verkehrsbetriebe gerichtete Umfrage, bei deren Rücklauf eine Auswertung von 32 Betrieben mit Haltestellen-Kaps dokumentiert werden konnte ${ }^{30}$. Insbesondere sollten die Vor- und Nachteile dieser Haltestellenform durch die Verkehrsbetriebe diskutiert werden. Dabei konnte festgestellt werden, dass mehrheitlich positive Erfahrungen mit dem Einsatz dieser Haltestellenform verbunden waren. Sofern jedoch noch keine Kap-Haltestellen in den Bediengebieten der befragten Verkehrsbetriebe zum Einsatz kamen, bestanden insbesondere Vorbehalte bzgl. der Auswirkungen auf den Verkehrsfluss im Haltestellenbereich. In einem weiteren Schritt wurden vier Haltestellen näher untersucht. Dabei lag der Fokus auf den Formen ${ }^{31}$ :

- Haltestellen-Kap (bei Straßenbahnen) und

- Haltestellen in Mittellage (Fahrgastwechsel auf der Fahrbahn bei Straßenbahnen)

Um das Verkehrsverhalten, wie auch den Verkehrsablauf, an den ausgewählten Haltestellen zu untersuchen, erfolgte die Erfassung der Fahrzeuggeschwindigkeiten wie auch eine Auswertung 
des Verkehrsgeschehens an Hand von Videoaufzeichnungen. Aus den Untersuchungen können die folgenden Kernaussagen zusammengefasst werden:

- Haltestellen-Kaps wirken auf Grund der optischen Einengung des Querschnitts geschwindigkeitsreduzierend

- die größten Konfliktpotentiale bestehen in:

- Querung der Fahrbahn von Fahrgästen (vor oder hinter dem ÖV)

- Überholen des ÖV durch IV

- Fahrten des IV in Gegenrichtung

Insgesamt können Haltestellen-Kaps als eine der sichersten Haltestellen Formen benannt werden, da die wesentlichen Konfliktsituationen:

- rechts Überholen des haltenden ÖV durch IV

- Queren der Fahrbahn durch die Fahrgäste zum haltenden ÖV

auf Grund ihrer baulichen Beschaffenheit nicht auftreten. Darüber hinaus werden weitere positive Effekte, wie die bessere Erkennbarkeit als auch die Nutzung für mobilitätseingeschränkte Personen, benannt.

\begin{tabular}{|l|l|l|l|}
\hline Titel: & \multicolumn{2}{|l|}{ Haltestellenformen an innerörtlichen Hauptverkehrsstraßen } \\
\hline Veröffentlicht: & 1994, BASt \\
\hline Autor(en): & Topp, Haag, Kupfer, Ackermann & Quellenangabe: & [TOPP, HAAG et al. 1994] \\
\hline
\end{tabular}

1994 erfolgte eine weitere Veröffentlichung der BASt unter dem Thema „Haltestellenformen an innerörtlichen Hauptverkehrsstraßen“. Ziel dieser Untersuchung war die „Bewertung der Sicherheit und des Verkehrsablaufs für alle Verkehrsteilnehmer [...] von ÖPNV-Haltestellen in Hauptverkehrsstraßen in Abhängigkeit von unterschiedlichen Randbedingungen“32.

Insgesamt wurden an Hand von Videoaufzeichnungen 24 Haltestellen für Busverkehr untersucht. Davon waren 10 Haltestellkaps, 10 Haltestellenbuchten und 4 Haltestellen am Fahrbahnrand. Ziel der Untersuchung war es, für die genannten Haltestellenformen Einsatzgrenzen und Ausschlusskriterien für verschiedene Parameter zur erarbeiten, wie bspw. Fahrstreifenanzahl und Verkehrsbelastung ÖPNV/ MIV, Lage der Haltestelle zu Knotenpunkten und LSA, Geschwindigkeit des MIV und Führung des Radverkehrs ${ }^{33}$

Eine Integration von Verkehrsunfallanzeigen in die Untersuchungen wird diskutiert jedoch nicht weiterverfolgt. Im Rahmen einer Stichprobe werden Unfallanzeigen bzgl. Ihres Inhalts untersucht jedoch nur wenige auswertbare Erkenntnisse für Unfälle mit Haltestellenbezug gewonnen. Die Ergebnisse dieser Untersuchung stützten sich daher im Wesentlichen auf Konfliktbetrachtungen.

Folgende Kernergebnisse der Forschungsarbeit können auf dieser Untersuchungsgrundlage zusammengefasst werden ${ }^{34}$ :

- Haltestellen in Seitenlage ohne Bucht weisen hohe Vorteile bei der Anfahrbarkeit durch den ÖV auf

2 [TOPP, HAAG et al. 1994], S. 25

[TOPP, HAAG et al. 1994], S. 25

44 [TOPP, HAAG et al. 1994], S. 56, S. 58f, S. 75 
- Die Haltestellenform hat einen wesentlichen Einfluss auf den Verkehrsablauf des MIV, wobei dies oft bereits auch mit weiteren Faktoren (wie der Verkehrsbelastung, dem Geschwindigkeitsniveau, der Haltestellen-Aufenthaltszeit, etc.) in Verbindung steht

- bei allen untersuchten Haltestellenformen ereignen sich Auffahrunfälle der Kfz durch Ein- und Ausfahrvorgänge bspw. aus der Bucht oder durch das verzögernde ÖV-Fahrzeug auf dem Fahrstreifen

- Auffahrunfälle lassen sich daher nur durch bauliche, betriebliche und organisatorische Maßnahmen (Geschwindigkeitsreduzierung, Signalisierung, etc.) beseitigen

- Bei Fahrbahnhaltestellen handelt es sich im Wesentlichen um Unfälle beim Überholen des auf der Fahrbahn haltenden Busses, welches sich durch einfache Maßnahmen unterbinden lässt

- Bei Busbuchten sind Unfälle durch ein- und ausparkende Fahrzeuge (u.a. Lieferverkehr) oder wendende Fahrzeuge zu beobachten

Damit ergibt sich folgende Gesamtbewertung der Haltestellenformen:

Tabelle 3-8: $\quad$ Bewertung der Haltestellenformen

Quelle: $\quad$ nach [TOPP, HAAG et al. 1994], S. 74

\begin{tabular}{|l|c|c|c|c|}
\hline \multicolumn{1}{|c|}{ Kriterium } & $\begin{array}{c}\text { Richtlinien- } \\
\text { gerechte Bucht }\end{array}$ & $\begin{array}{c}\text { Unterdimensio- } \\
\text { nierte Bucht }\end{array}$ & Kap & Fahrbahnrand \\
\hline Verkehrssicherheit & 0 & 0 & $\left.+^{*}\right)$ & $\left.+^{*}\right)$ \\
\hline Anfahrbarkeit & + & - & + & + \\
\hline Fußgänger und Radfahrer & - & 0 & 0 & 0 \\
\hline Verkehrsablauf MIV & + & - & 0 & 0 \\
\hline Geschwindigkeit & - & 0 & 0 & 0 \\
\hline Haltestellenaufenthaltszeit & 0 & 0 & 0 & 0 \\
\hline Beschleunigung/ Verzögerung & 0 & 0 & + & + \\
\hline Straßenraumgestaltung & - & 0 & + & + \\
\hline Kosten & - & - & + & + \\
\hline Gesamtbeurteilung & 0 & + & + \\
\hline
\end{tabular}

*): bei Verhinderung des Überholens; +: Vorteil der Haltestellenform; o: weder Vor- noch Nachteil der Haltestellenform;

-: Nachteil der Haltestellenform

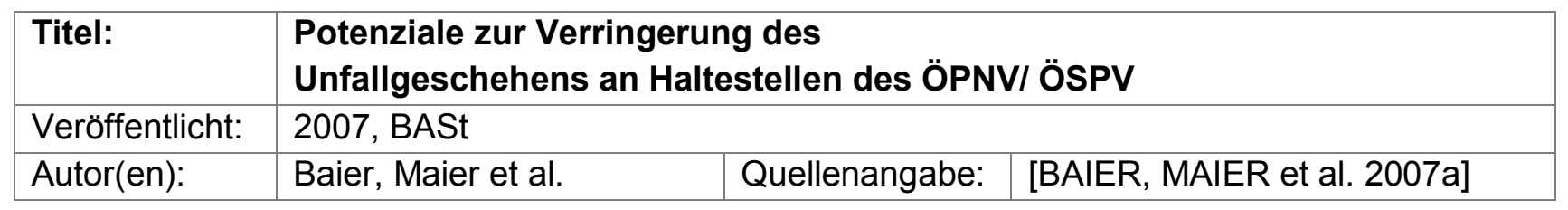

Im Jahr 2007 folgte eine weitere Untersuchung der BASt: „Potenziale zur Verringerung des Unfallgeschehens an Haltestellen des ÖPNVI ÖPSV“. Ziel dieser Untersuchung war es noch „bestehende Wissenslücken zum Verkehrssicherheitspotential an innerörtlichen Haltestellen des straßen- und schienengebundenen öffentlichen Personennahverkehrs“"35 aufzuzeigen und zu schließen. Der Fokus der Forschungsarbeit liegt dabei auf den betrachteten Haltestellenformen und den sich daraus ergebenden Unfallrisiken. 
Folgende methodische Ansätze wurden bei dem Forschungsvorhaben angewandt:

- Auswertung der bundesweiten Verkehrsunfallstatistik

- Auswertung des Unfallgeschehens an 2.457 Haltestellen (Bus, Straßenbahn, kombiniert) mit insgesamt 765 über drei Jahre polizeilich registrierten Unfällen

- Vertiefende Untersuchung von je einer Kaphaltestelle und Fahrbahnrandhaltestelle und je zwei "Fahrbahnhaltestellen" mit StVO-Regelung ${ }^{36}$ und mit Zeitinsel

Im Gegensatz zu bisherigen Untersuchungen beruhen die Ergebnisse damit im Wesentlichen auf einer Auswertung polizeilich erhobener Verkehrsunfalldaten.

Untersucht wurden Unfälle mit Personenschaden über drei Kalenderjahre mit dem Sondermerkmal „Haltestelle" bzw. "Beteiligung von ÖPNV-Fahrzeugen". Für eine Berücksichtigung der Unfallschwere wurden angepasste Unfallkosten ermittelt und bei der Bewertung der Unfälle zugrunde gelegt.

Die folgenden wesentlichen Erkenntnisse konnten aus der bundesstatistischen Auswertung gewonnen werden ${ }^{37}$ :

- Der Anteil der Unfälle mit Personenschaden und mit schwerem Personenschaden ist an Haltestellen höher als bei allen Unfällen

- Als häufigster Unfalltyp an Haltestellen können Überschreiten-Unfälle festgestellt werden

- Haltestellen beeinflussen den Verkehrsablauf maßgeblich; dies zeigt sich an der Häufigkeit auftretender Unfälle im Längsverkehr

- In etwa der Hälfte der Fälle sind Fußgänger am Unfallgeschehen, mit einem Anteil von $75 \%$ an den Unfallkosten, beteiligt.

Über die Auswertung der Verkehrsunfallstatistik hinaus erfolgte wie o.g. eine Auswertung des Unfallgeschehens an 2.457 Haltestellen in den drei Städten Düsseldorf, Leipzig, Zwickau und dem Landkreis Mayen-Koblenz ${ }^{38}$. Es wurden sowohl der räumliche Bezug „Haltestellen-Bereich" (in Abhängigkeit der Haltestellenform) als auch ein Annährungsbereich von $50 \mathrm{~m}$ festgelegt. Folgend wurde eine Auswertung hinsichtlich der Unfalltypen, der Verkehrsbeteiligung, wie auch der Charakteristik der Unfallstelle vorgenommen.

Es erfolgte eine Zuordnung der Haltestellen zu den in Tabelle 3-9 erkennbaren Grundtypen und die Erhebung zusätzlicher Merkmale nach Tabelle 3-10.

Ein- und Aussteiger queren die Fahrbahn und haben nach §20 (2) StVO Vorrang

[BAIER, MAIER et al. 2007a], S. 16ff

[BAIER, MAIER et al. 2007a], S. $23 f$ 
Tabelle 3-9: Typisierung der Haltestellen bzw. Teilhaltestellen hinsichtlich Haltestellenausführung und Lage der Warteflächen im Straßenquerschnitt

Quelle: $\quad$ [BAIER, MAIER et al. 2007a], S. 29

\begin{tabular}{|l|c|c|c|}
\hline Grundtyp Teilhaltestelle & Bus & Strab & Bus+Strab \\
\hline Fahrbahnrand & $\bullet$ & $\bullet$ & $\bullet$ \\
\hline Kap & $\bullet$ & $\bullet$ & $\bullet$ \\
\hline Bucht & $\bullet$ & - & - \\
\hline Fahrbahn, StVO & - & $\bullet$ & $\bullet$ \\
\hline Fahrbahn, Zeitinsel & - & $\bullet$ & $\bullet$ \\
\hline Seitenbahnsteig & $\bullet$ & $\bullet$ & $\bullet$ \\
\hline Mittelbahnsteig & $\bullet$ & $\bullet$ & - \\
\hline ÖPNV-Trasse** in Seitenlage & $\bullet$ & $\bullet$ & $\bullet$ \\
\hline * Wartefläche im Seitenraum, Bus hält am Fahrbahnrand \\
** Bussonderfahrstreifen und/oder besonderer Bahnkörper \\
\hline
\end{tabular}

Tabelle 3-10: $\quad$ Erhobene Merkmalskategorien und Differenzierungsmerkmale zu den untersuchten Haltestellen in den Fallbeispielen

Quelle: $\quad$ [BAIER, MAIER et al. 2007a], S. 32

\begin{tabular}{|c|c|c|}
\hline Merkmalskategorie & \multicolumn{2}{|l|}{ Differenzierungsmerkmale } \\
\hline \multirow{5}{*}{$\begin{array}{l}\text { Sicherung } \\
\text { (Überquerungsstellen) }\end{array}$} & Keine Sicherung & \\
\hline & \multirow{3}{*}{ Sicherung an einem Ende der Wartefläche } & Furt an Lichtsignalanlage (LSA) \\
\hline & & Mittelinsel/-streifen (MI/MS) \\
\hline & & Fußgängerüberweg FGÜ) \\
\hline & Sicherung an beiden Enden der Wartefläche & Kombinationen der o. g. Sicherungselemente \\
\hline \multirow{3}{*}{ Radverkehrsanlagen } & \multicolumn{2}{|c|}{ Fahrbahn (Mischverkehr, Radfahrstreifen, Schutzstreifen) } \\
\hline & \multicolumn{2}{|c|}{ Radweg, Führung zwischen Fahrbahn und Wartefläche/Witterungsschutz } \\
\hline & \multicolumn{2}{|c|}{ Radweg, Führung hinter Wartefläche/Witterungsschutz } \\
\hline \multirow{5}{*}{$\begin{array}{l}\text { Nutzungen } \\
\text { (Haltestellenumfeld) }\end{array}$} & \multicolumn{2}{|l|}{ Kerngebiet, Geschäftsstraße } \\
\hline & \multicolumn{2}{|l|}{ Mischnutzung } \\
\hline & \multicolumn{2}{|l|}{ Wohnnutzung } \\
\hline & \multicolumn{2}{|l|}{ Gewerbe, Industrie } \\
\hline & \multicolumn{2}{|l|}{ Grün-/Freifläche, Wald } \\
\hline
\end{tabular}

Aufgrund beschriebener Unfallauswertung können folgende Feststellungen getroffen werden ${ }^{39}$ :

- Unfälle an Haltestellen weichen deutlich in einigen Merkmalen (Unfalltyp, Unfallart, Beteiligungen) vom Unfallgeschehen der gesamtstädtischen Ebene ab.

- Bei Unfällen an Haltestellen liegt der Anteil der Fußgänger deutlich und der Anteil von Straßenbahn und Bus als Hauptverursacher leicht über der gesamtstädtischen Ebene

- Das Unfallgeschehen in Annährungsbereichen der Haltestelle verhält sich ähnlich den Unfallgeschehen der gesamtstädtischen Ebene.

Im Rahmen der Bewertung der Verkehrssicherheit auf Basis der haltestellenbereichsbezogenen Unfallkosten nach Haltestellenformen können darüber hinaus folgende wesentliche Ergebnisse zusammengefasst werden ${ }^{40}$ : 
- Bushaltestellen sind sicherer als Straßenbahnhaltestellen und kombinierte Haltestellen

- Bushaltestellen am Fahrbahnrand (einschließlich Kap-Lösungen) sind sicherer als Busbuchten.

- Bei Straßenbahnhaltestellen ergibt sich folgende Rangliste nach Verkehrssicherheit (beginnend mit der sichersten Haltestellenform, in Klammern die durchschnittlichen Unfallkosten):

1. Kaphaltestelle $(4.229 € / a)$

2. Fahrbahnrand (5.629 €/a)

3. Fahrbahnhaltestelle mit StVO -Regelung ${ }^{41}$ (6.138 €/a) (Haltestelle in Mittellage, Warteflächen am äußeren Fahrbahnrand, StVO -Regelung)

4. Fahrbahnhaltestelle mit Zeitinsel (10.934 €/a) (Haltestelle in Mittellage, Warteflächen am äußeren Fahrbahnrand, Zeitinsel)

5. Seitenbahnsteig (13.686 €/a) (Haltestelle in Mittellage, Warteflächen in Mittellage)

Haltestellen mit den Gleisen in Mittellage haben sich als vergleichsweise unsicher herausgestellt. Bei Haltestellentyp "Seitenbahnsteig" ist Unfalltyp 4 (Überschreiten, 41\%) und Typ 6 (Längsverkehr, 19\%) dominant.

Die höheren Unfallkosten des Typs „Fahrbahn (Zeitinsel)” gegenüber dem Typ "Fahrbahn (StVO)" resultiert aus höheren Werten bei allen Unfalltypen (Ausnahme Typ 6, Unfall im Längsverkehr), insbesondere bei Unfalltyp 1 (Fahrunfall), Unfalltyp 2 (Abbiege-Unfall) und Unfalltyp 3 (Einbiegen/ Kreuzen).

Die vertiefende Untersuchung von zwei "Fahrbahnhaltestellen" mit StVO-Regelung ergab, folgende potenzielle zusätzliche Konfliktgründe bei diesem Haltestellentyp:

- zu knappe Sperrzeiten der Zeitinsel

- mangelnde Erkennbarkeit des Signalgebers

- Laufeinsteiger über die Gegenfahrbahn

Aus der Untersuchung werden Empfehlungen abgeleitet:

- bei Realisierung des Typs „Fahrbahnrand/Kap” verstärkte Berücksichtigung der Aspekte: Sicherung von Fahrbahnquerungen, Ordnung des ruhenden KfZ-Verkehrs, Radverkehrsführung

- Neubewertung des Typs „Fahrbahn“ und hier wiederum der Variante „StVO“ in VwV-StVO und Regelwerken mit dem Ziel einer Priorisierung des Typs „Fahrbahn“ gegenüber „Seitenbahnsteig“ sowie der Variante „StVO“ gegenüber „Zeitinsel“

- Konkretisierung der Einsatzkriterien für Zeitinseln, insbesondere zur stärkeren Sicherung von Ein-/Aussteigern durch frühzeitigen Sperrzeitbeginn, zur Sicherung frei querender Fußgänger, zur Verbesserung der Erkennbarkeit des Signalgebers, zur Verhinderung riskanter Fahrmanöver im Kfz-Verkehr

- Betonung der besonderen Verkehrssicherheitswirkung einer baulichen Anhebung der Fahrbahn im Haltestellenbereich in VwV-StVO und Regelwerken

- bewertende Untersuchung einer zweiten signalisierten Furt im Haltestellenbereich zur Sicherung des Querungsbedarfs hinter dem Fahrzeugheck als Alternative zur Zeitinsel

41 Ein- und Aussteiger queren die Fahrbahn und haben nach $\$ 20$ (2) StVO Vorrang 


\subsubsection{Sicherheitsuntersuchungen auf der freien Strecke}

\begin{tabular}{|l|l|l|l|}
\hline Titel: & \multicolumn{2}{|l|}{$\begin{array}{l}\text { Verkehrssicherheitsbelange bei der Bevorrechtigung von Bussen und } \\
\text { Bahnen im Stadtverkehr }\end{array}$} \\
\hline Veröffentlicht: & 1998 & Quellenangabe: & [WILMS 1998] \\
\hline Autor(en): & Wilms &
\end{tabular}

Beschleunigungsmaßnahmen im ÖPNV haben i.A. räumliche Einschränkungen (Zuweisung besonderer Verkehrsflächen) als auch zeitliche Einschränkungen (Priorisierung an Knotenpunkten, Veränderung der Freigabezeiten) an Streckenabschnitten, Knotenpunkten und Haltestellen für die übrigen Verkehrsteilnehmer zur Folge.

Im Rahmen der Untersuchung „Verkehrssicherheitsbelange bei der Bevorrechtigung von Bussen und Bahnen im Stadtverkehr" wurden auf Basis einer Literaturrecherche und Expertengesprächen in 18 deutschen Städten unter Beteiligung der zuständigen Verkehrsbetriebe, Planungsämter und Polizeidienststellen verkehrssicherheitsrelevante Problemschwerpunkte benannt ${ }^{42}$ :

Streckenabschnitte:

- Überquerungsstellen ÖPNV-Fahrstreifen in Mittellage

- Einbiege- und Abbiegeverkehre ÖPNV-Fahrstreifen in Seitenlage

- Zulassung anderer Verkehrsarten auf Busfahrstreifen

- Überquerbarkeit bestimmter Ausbauformen von Bahnkörpern

Knotenpunkte:

- Wartezeiten im Individualverkehr an Knotenpunkten mit LSA

- Signalisierung der Furten für Fußgänger- und Radverkehr bei ÖPNV-Fahrstreifen in Mittel- oder Seitenlage

- Vorfahrtregelung bei abknickender Linienführung der Straßenbahnen an Knotenpunkten ohne LSA

Haltestellen

- Erreichbarkeit der Haltestelle bei Haltestellen in Mittellage

- Radverkehrsführung an Haltestellen

- Radverkehrsführung an Haltestellen im Zuge von Fahrstreifen mit gemeinsamer Nutzung durch Bus- und Radverkehr

Folgend werden die daraus gewonnenen, wesentlichen Erkenntnisse zusammengefasst ${ }^{43}$ :

\section{Streckenabschnitte}

- Straßenbündige Bahnkörper können hinsichtlich der Führung des Kfz-Verkehrs zu einem erhöhten Unfallrisiko beitragen (Überholvorgänge im Gleisbereich, Verschwenken im Fahrstreifen, Abbiegefahrt, etc.)

- ein großer Anteil von Fußgängern nutzen Bahnkörper in Mittellage als Überquerungshilfe; dies ist weniger abhängig von der Ausbauform als vielmehr von den Umfeldnutzungen

42 [WILMS 1998], S. 128

43 [WILMS 1998] , S.6ff, S. 130 
- Überschreiten-Unfälle (auch zwischen Kfz und Fußgänger) bestimmen maßgeblich das Unfallgeschehen bei Bahnkörpern in Mittellage

- fehlende Integration des Nutzungsanspruches „lineares queren“ bei der Straßenraumgestaltung, insbesondere problematisch in geschäftlich genutzten Innerortsstraßen

Knotenpunkte

- Vermehrt treten Abbiegeunfälle des nicht gesichert geführten Linksabbiegers sowie Rotlichtverstöße bei Bahnkörpern in Mittellage auf.

- Hohe Wartezeiten des MIV bei ÖPNV-Beschleunigungsmaßnahmen können sich kritisch auf das Verkehrsgeschehen auswirken

- Bei abknickender Führung der Straßenbahnen an Knotenpunkten ohne LSA (mit Bevorrechtigung der Straßenbahn nach zulässiger Regelung in der ehemaligen DDR) traten vermehrt Auffahrunfälle zwischen Kfz auf

Haltestellen

- Die Ausführungen zum Themenbereich Haltestellen stützt sich im Wesentlichen auf die unter Kapitel 3.2.1 beschriebene Literatur und werden daher an dieser Stelle nicht weiter ausgeführt.

Die Inhalte dieser Forschungsarbeit weisen insbesondere auf Gefährdungspotentiale im Umgang mit Beschleunigungsmaßnahmen des ÖPNV hin. Das Aufzeigen von Maßnahmen zur Verbesserung der beschriebenen Situationen wird jedoch nur in Teilen umgesetzt.

\begin{tabular}{|c|c|c|c|}
\hline Titel: & \multicolumn{3}{|c|}{$\begin{array}{l}\text { Sicherheitsgrad von Stadtstraßen mit und ohne } \\
\text { schienengebundenem ÖPNV }\end{array}$} \\
\hline Veröffentlicht: & 2007, GDV, unveröffentlicht & & \\
\hline Autor(en): & Baier, Maier, Aurich, Klemps & Quellenangabe: & [BAIER, MAIER et al. 2007b] \\
\hline
\end{tabular}

Im Jahr 2007 wurde erstmals eine Vergleichsuntersuchung bzgl. des Sicherheitsgrades von Stadtstraßen mit und ohne schienengebundenen ÖPNV durchgeführt. Dazu wurden für die vier Beispielstädte Berlin (Ost), Dresden, Düsseldorf und Leipzig knotenpunktfreie Strecken mit und ohne Straßenbahnverkehr vergleichend untersucht. Als knotenpunktfreie Strecken gelten dabei Streckenabschnitte zwischen Knotenpunkten kreuzender Hauptverkehrsstraßen. Knotenpunkte mit Erschließungsstraßen werden als Anschlussknoten als Teil der knotenpunktfreien Streckenabschnitte gewertet. ${ }^{44}$

Als Untersuchungsnetz mit schienengebundenem ÖPNV wurde das komplette Straßennetz mit schienengebundenem ÖPNV einer Stadt ausgewählt.

Es wurden acht typische Querschnitte der Straßen mit Straßenbahnen unterschieden ${ }^{45}$.

- I: vierstreifige Straße mit besonderem Bahnkörper (Sicherheitsraum $\geq 1 \mathrm{~m}$ )

- II: vierstreifige Straße mit besonderem Bahnkörper (Sicherheitsraum $<1 \mathrm{~m}$ )

- III: zweistreifige Straße mit besonderem Bahnkörper (Sicherheitsraum < $1 \mathrm{~m}$ )

- IV: vierstreifige Straße mit straßenbündigem Bahnkörper

44 [BAIER, MAIER et al. 2007b], S. 12

45 [BAIER, MAIER et al. 2007b], S. $21 \mathrm{ff}$ 
- V: dreistreifige Straße mit straßenbündigem Bahnkörper (später verworfen)

- VI: zweistreifige Straße mit straßenbündigem Bahnkörper

- VII: besonderer Bahnkörper außerhalb der Fahrbahn

- VIII: zweistreifige Straße mit abmarkierten besonderem Bahnkörper (später verworfen)

Die Auswahl der Referenzstrecken erfolgte über die Kfz-Verkehrsstärke. Die Verkehrsstärken der Strecken mit und ohne Straßenbahnen wurde in 7 Belastungsklassen eingeteilt und je Klasse wurden Referenzstrecken mit ungefähr gleicher Gesamtstreckenlänge ausgewählt ${ }^{46}$.

Als Querschnittstypen für Straßen ohne Straßenbahn wurden die folgenden Typen unterschieden ${ }^{47}$ :

- zweistreifige Straße

- vierstreifige Straße

- vierstreifige Straße mit baulicher Mitteltrennung

Alle Querschnittstypen, die sich nicht in diese Klassifizierung eingliedern lassen, werden in den Kategorien „Sonstige Querschnitte" mit und ohne Straßenbahn zusammengefasst.

Die Bewertung des Unfallgeschehens erfolgt an Hand der Unfallkostensätze (Preisstand: 2000) und den daraus resultierenden durchschnittlichen Unfallkostenraten (UKR) und Unfallkostendichten (UKD) ${ }^{48}$. Die aus Tabelle 3-11 zu erkennenden Unfallkenngrößen nach Höhe der Unfallkostenrate sortiert wurden ermittelt:

Tabelle 3-11: Rangfolge der Querschnittstypen mit Straßenbahn nach Unfallkostenraten Quelle: [BAIER, MAIER et al. 2007b], S. 65

\begin{tabular}{|c|c|c|}
\hline Querschnittstyp VII & Querschnittstyp II & Querschnittstyp I \\
\hline$+\quad t$ & $+b$ & +1 \\
\hline $\begin{aligned} \mathrm{UKR}_{1-4} & =22[\epsilon /(1.000 \mathrm{Kfz}-\mathrm{km})] \\
\mathrm{UKD}_{1-4} & =201[1.000 € / \mathrm{km} \cdot \mathrm{a})]\end{aligned}$ & $\begin{aligned} U_{K R} R_{1-4} & =28[\epsilon /(1.000 \mathrm{Kfz}-\mathrm{km})] \\
U K D_{1-4} & =301[1000 € / \mathrm{km} \cdot \mathrm{a})]\end{aligned}$ & $\begin{aligned} \mathrm{UKR}_{1-4} & =37[\epsilon /(1.000 \mathrm{Kfz}-\mathrm{km})] \\
\mathrm{UKD}_{1-4} & =380[1.000 € / \mathrm{km} * \mathrm{a})]\end{aligned}$ \\
\hline Querschnittstyp III & Querschnittstyp IV & Querschnittstyp VI \\
\hline$t$ & $++1+1$ & + \\
\hline $\begin{aligned} \mathrm{UKR}_{1-4} & =39[\epsilon /(1.000 \mathrm{Kfz}-\mathrm{km})] \\
\mathrm{UKD}_{1-4} & =216[1.000 € / \mathrm{km} \cdot \mathrm{a})]\end{aligned}$ & $\begin{aligned} \mathrm{UKR}_{1-4} & =41[\Theta /(1.000 \mathrm{Kfz}-\mathrm{km})] \\
\mathrm{UKD}_{1-4} & =277[1.000 € / \mathrm{km} \cdot \mathrm{a})]\end{aligned}$ & $\begin{aligned} \mathrm{UKR}_{1-4} & =57[\Theta /(1.000 \mathrm{Kfz}-\mathrm{km})] \\
\mathrm{UKD} & =211[1.000 € / \mathrm{km} \cdot \mathrm{a})]\end{aligned}$ \\
\hline
\end{tabular}

46 [BAIER, MAIER et al. 2007b], S. 24

47 [BAIER, MAIER et al. 2007b], S. 23

48 [BAIER, MAIER et al. 2007b], S. 40 
Für die Betrachtung der Vergleichsquerschnitte ohne Straßenbahnen wurden die folgenden Unfallkenngrößen ermittelt ${ }^{49}$ :

Tabelle 3-12: $\quad$ Rangfolge der Querschnittstypen ohne Straßenbahn nach Unfallkostenraten

Quelle:

[BAIER, MAIER et al. 2007b], S. 70

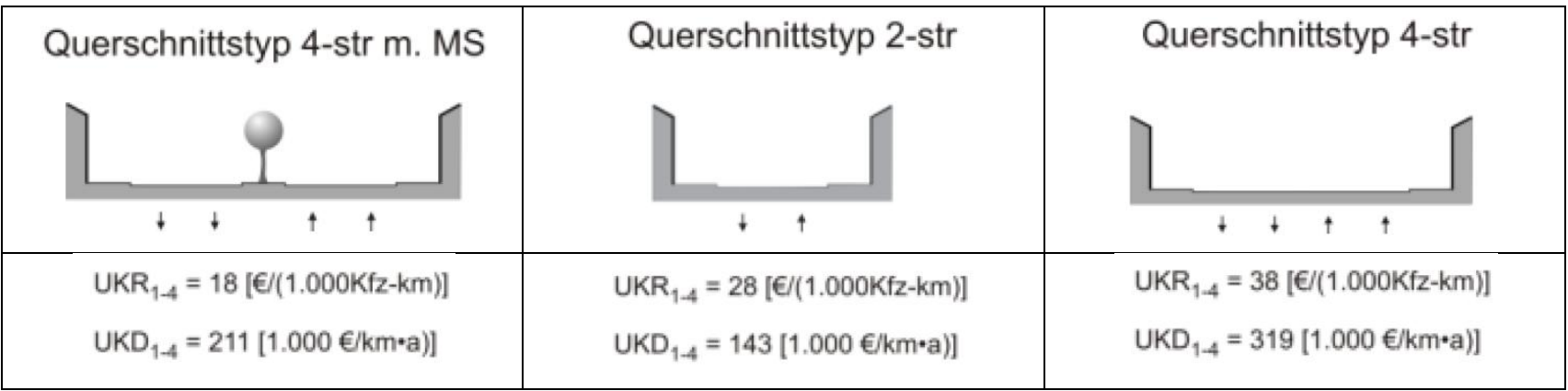

In der folgenden Zusammenführung der betrachteten Querschnittstypen mit und ohne Straßenbahn werden Erkenntnisse bzgl. des Unfallgeschehens auf Streckenabschnitten als auch auf Knotenpunkten ausgeführt ${ }^{50}$ :

Streckenabschnitte:

- Straßen ohne eine Führung des schienengebundenen ÖPNV weisen in etwa $30 \%$ niedrigere UKR auf.

- Zwei- und vierstreifige Querschnitte reagieren verstärkt negativ auf die Integration der Führung von Straßenbahnen.

- Straßen mit Straßenbahn weisen bei zunehmender Verkehrsstärke einen Rückgang der UKR und eine Zunahme der UKD auf

- Straßen ohne Straßenbahn weisen bei zunehmender Verkehrsstärke sowohl einen Rückgang der UKR als auch der UKD auf.

Knotenpunkte:

- Knotenpunkte ohne Führung von Straßenbahnen weisen i.d.R. eine ca. 25\% geringere UKR wie auch UKD auf

- an Knotenpunkten mit Straßenbahnen kann eine Zunahme der Unfalltypen ÜberschreitenUnfälle und Abbiegen/Kreuzen-Unfälle verzeichnet werden.

Über diese Erkenntnisse hinaus wurden ebenfalls abschließend die Wirkung von Radverkehrsführungen als auch die Anlagen des ruhenden Verkehrs betrachtet. Festgestellt werden konnte, dass ${ }^{51}$ :

- Radverkehr: im Zuge einer Einbindung des Radverkehrs der Sicherheitsgrad auf den unterschiedlichen Querschnitten maßgeblich durch die Ausstattung der Radverkehrsanlagen (beidseitig, einseitig, ohne) bestimmt wird.

- Ruhender Verkehr: Querschnitte mit Parken niedrigere Sicherheitsgrade aufweisen.

- Rad- und Ruhender Verkehr: Querschnitte ohne Radverkehrsanlagen aber mit Anlagen des ruhenden Verkehrs die ungünstigsten Sicherheitsgrade aufweisen. 


\subsection{Rechtliche Vorgaben, Regelwerke und Richtlinien}

Für die Untersuchung der Verkehrs- und Straßenräume werden neben der in Kapitel 3.2 benannten und ausgewerteten Fachliteratur zusätzlich geltende rechtliche Vorgaben, Richtlinien und Regelwerke zugrunde gelegt.

\subsubsection{Rechtliche Vorgaben}

\begin{tabular}{|l|l|}
\hline Titel: & Verordnung über den Bau und Betrieb der Straßenbahnen \\
\hline Veröffentlicht: & 1987, aktualisierte Fassung vom 08.11.2007 \\
\hline Quellenangabe: & [BOStrab 2007] \\
\hline
\end{tabular}

Die BOStrab regelt im Sinne von $\S 4$ und $\S 57$ des PBefG den Bau und Betrieb von Straßenbahnen, Hoch- und Untergrundbahnen, Einschienen- und Schwebebahnen.

allgemeine Aussagen zur Sicherheit:

- § 2 (1): Betriebsanlagen und Fahrzeuge müssen so beschaffen sein, dass sie den Anforderungen der Sicherheit und Ordnung genügen.

- § 3 (1): Betriebsanlagen und Fahrzeuge müssen so gebaut sein, dass ihr verkehrsüblicher Betrieb niemand schädigt oder mehr als unvermeidbar gefährdet oder behindert.

Aussagen zur Typisierung und sicherheitsrelevante Aussagen zur Infrastruktur sind im vierten Abschnitt "Betriebsanlagen" enthalten:

- § 16 (4): Differenzierung in straßenbündiger, besonderer und unabhängiger Bahnkörper

- § 16 (8): Bei Fußgängerüberwegen über einen besonderen Bahnkörper müssen zwischen Bahnkörper und Straßenfahrbahnen Schutzinseln für Fußgänger oder LSA-Steuerung vorhanden sein.

- $§ 18$ (1): qualitative Definition des Lichtraums; aufgrund Geltungsbereich für versch. Fahrzeuge keine Maßangaben (nähere Angaben in BOStrab-Lichtraum-Richtlinie 1996)

- § 19: qualitative und quantitative Definition des Sicherheitsraumes (0,7 m breit, 2,0 m hoch, lotrecht); im Verkehrsraum gilt dieser mit als Sicherheitsraum; bei Haltestellenflächen $>0,5 \mathrm{~m}$ über dem Bahnkörper muss Sicherheitsraum auf gegenüberliegender Seite oder unter Bahnsteig (0,7 m Tiefe x 0,7 $\mathrm{m}$ Höhe) angeordnet sein

- § 20: Definition von (mit Andreaskreuz gekennzeichnete) Bahnübergängen und Festlegung der technischen Sicherung: ohne Sicherung bei einem DTV $<100 \mathrm{Kfz} / \mathrm{d}$ und bei übersichtlichen Fuß- oder Radwegeübergängen; sonst Lichtzeichenanlage mit ggf. zusätzlichen Halbschranken)

- § 16 (6): Besondere Bahnkörper liegen im Verkehrsraum öffentlicher Straßen, sind jedoch vom übrigen Verkehr durch Bordsteine, Leitplanken, Hecken, Baumreihen oder andere ortsfeste Hindernisse getrennt.

- § 31 (2): "Zu- und Abgänge in Haltestellen müssen sicher und bequem sein"

- § 31 (6): Mindestbreite von Bahnsteigen im Verkehrsraum öffentlicher Straßen: 1,5 m (2,0 m)

Der fünften Abschnitt "Fahrzeuge" enthält Aussagen zu Fahrzeugmaßen (2,65 m bei straßenabhängigen Bahnen) und zur Bremsverzögerung. 
Für den Betrieb wurden folgende wesentliche Aussagen getroffen:

- § 49 (1): ein Zug darf einem anderen nur in einem solchen Abstand folgen, dass er auch bei ungünstigen Betriebsverhältnissen, insbesondere bei unvermutetem Halten des vorausfahrenden Zuges, rechtzeitig zum Halten gebracht werden kann. Dieser Abstand muss 1. bei Fahren auf Sicht vom Fahrzeugführer bewirkt werden,

2. bei Fahren auf Zugsicherung durch Zugsicherungsanlagen nach § 22 gewährleistet sein.

- § 49 (2): Auf Sicht dürfen nicht fahren

1. Züge unabhängiger Bahnen, (Hoch- und Untergrundbahnen, Schwebebahnen)

2. Züge straßenabhängiger Bahnen

a) bei Streckenhöchstgeschwindigkeit über $70 \mathrm{~km} / \mathrm{h}$,

b) in Tunneln.

- Nach § 50 legt die zulässige Geschwindigkeit die Technische Aufsichtsbehörde fest. Bei straßenbündigen Bahnkörpern gilt die zulässige Geschwindigkeit des übrigen Verkehrs.

Züge auf straßenbündigen Bahnkörpern dürfen nach $\S 70$ maximal 75 m lang sein.

\begin{tabular}{|l|l|}
\hline Titel: & Straßenverkehrs-Ordnung \\
\hline Veröffentlicht: & 06.03.2013, Neufassung \\
\hline Quellenangabe: & [StVO 2013] \\
\hline
\end{tabular}

Die StVO regelt das Verhalten bei Teilnahme im Straßenverkehr. Sie enthält allgemeine Aussagen zu den Grundregeln, Warnzeichen, Beleuchtung, Verhalten bei Unfall u.ä. und gelten damit auch für Straßenbahnführer. Konkrete Aussagen sind im Allgemeinen nur zu straßenbündigen Bahnkörpern gegeben:

- § 2 (3): Fahrzeuge, die in der Längsrichtung einer Schienenbahn verkehren, müssen diese, soweit möglich, durchfahren lassen

- § 5 (2): Schienenfahrzeuge sind i.A. rechts zu überholen; nur bei Platzmangel oder auf Fahrbahnen für eine Richtung dürfen Schienenfahrzeuge auch links überholt werden

- § 9 (1): Wer nach links abbiegen will, darf sich auf längs verlegten Schienen nur einordnen, wenn kein Schienenfahrzeug behindert wird.

- § 9 (3): Wer abbiegen will, muss entgegenkommende Fahrzeuge durchfahren lassen, Schienenfahrzeuge auch dann, wenn sie auf oder neben der Fahrbahn in der gleichen Richtung fahren

- § 12 (4): Im Fahrraum von Schienenfahrzeugen darf nicht gehalten werden

- § 19 Bahnübergänge: Schienenfahrzeuge haben an Bahnübergängen mit Andreaskreuz sowie über Fuß-, Feld-, Wald- oder Radwegen Vorrang

- § 25 (5): Gleisanlagen, die nicht zugleich dem sonstigen öffentlichen Straßenverkehr dienen, dürfen nur an den dafür vorgesehenen Stellen betreten werden.

- § 26: An Fußgängerüberwegen haben Fahrzeuge mit Ausnahme von Schienenfahrzeugen den zu Fuß Gehenden [...] das Überqueren der Fahrbahn zu ermöglichen."

- § 37 Lichtzeichen: Für Schienenbahnen können besondere Zeichen, auch in abweichenden Phasen, gegeben werden 


\begin{tabular}{|l|l|}
\hline Titel: & Allgemeine Verwaltungsvorschrift zur Straßenverkehrsordnung \\
\hline Veröffentlicht: & 26.01.2001, aktualisierte Fassung vom 17.07.2009 \\
\hline Quellenangabe: & {$[$ VwV-StVO 2009] } \\
\hline
\end{tabular}

Die VwV-StVO regelt die Umsetzung der StVO durch die Straßenverkehrsbehörden und Straßenbauämter. Folgende darin enthaltene Anmerkungen zu den einzelnen Paragraphen der StVO sind mit Bezug zum Straßenbahnbetrieb im öffentlichen Verkehrsnetz:

- zu § 2 (3): Wo es geboten ist, den übrigen Fahrverkehr vom Schienenraum fernzuhalten, kann das durch bauliche Maßnahmen und Verkehrseinrichtungen (Bordsteinen, Fahrstreifenbegrenzungen (Zeichen 295), Sperrflächen (Zeichen 298), Geländer oder Absperrgeräte) erreicht werden

- zu § 8 Vorfahrt:

- An Kreuzungen sollte der Grundsatz "Rechts vor Links" nur gelten, wenn keine der Straßen, etwa durch Straßenbahngleise, den Eindruck einer übergeordneten Straßen macht

- Bei der Vorfahrtregelung sind die Interessen der öffentlichen Verkehrsmittel besonders zu berücksichtigen; Kann einer Straße, auf der eine Schienenbahn verkehrt, die Vorfahrt durch Verkehrszeichen nicht gegeben werden, so ist eine Regelung durch Lichtzeichen erforderlich; keinesfalls darf auf einer solchen Kreuzung die Regel "Rechts vor Links" gelten.

- zu § 26: Im Zuge von Straßen mit Straßenbahnen ohne eigenen Bahnkörper sollen Fußgängerüberwege nicht angelegt werden. Fußgängerüberwege über Straßen mit Schienenbahnen auf eigenem Bahnkörper sollen an den Übergängen über den Gleisraum mit versetzten Absperrungen abgeschrankt (Z-Übergang) werden.

- Zu § 37: ein Grünpfeil (LSA) darf nicht verwendet werden, wenn beim Rechtsabbiegen Gleise von Schienenfahrzeugen gekreuzt oder befahren werden müssen

- zu Zeichen 201 Andreaskreuz:

- i.A. Andreaskreuz auf Bahnkörpern außerhalb des öffentlichen Verkehrsraums notwendig

- bei schon mäßigem Verkehr oder Vzul > $50 \mathrm{~km} / \mathrm{h}$ ist eine LSA zu erwägen

- auch an Bahnübergängen von Feld- und Waldwegen sind Andreaskreuze dann erforderlich, wenn der Bahnübergang nicht ausreichend erkennbar oder die Übersicht nicht gegeben ist.

- An LSA Knotenpunkten, muss auch der Straßenbahnverkehr mittels LSA geregelt werden. Es ist stets zu erwägen, ob der die Schienen kreuzende Abbiegeverkehr gleichfalls durch Lichtzeichen zu regeln oder durch gelbes Blinklicht mit dem Sinnbild einer Straßenbahn zu warnen ist.

- zu Zeichen 205 Vorfahrt gewähren:

- in Ausnahmefällen wenn eine Bevorrechtigung der Schienenbahn auf andere Weise nicht möglich ist (Stop, LSA)

- bei Kreuzung von Kreisverkehrsplätzen durch Schienenbahnen oder bei Gleisschleifen

- zu Zeichen 245 Bussonderfahrstreifen:

- Taxen sollen grundsätzlich auf Sonderfahrstreifen zugelassen werden; dies gilt nicht für Sonderfahrstreifen im Gleisraum von Schienenbahnen. 


\subsubsection{Regelwerke und Richtlinien}

\begin{tabular}{|l|l|}
\hline Titel: & Richtlinien für die Anlage von Stadtstraßen \\
\hline Veröffentlicht: & 2006, FGSV \\
\hline Quellenangabe: & [RASt 2006] \\
\hline
\end{tabular}

Die RASt behandeln den Entwurf und die Gestaltung von Erschließungsstraßen, angebauten Hauptverkehrsstraßen und anbaufreien Hauptverkehrsstraßen mit plangleichen Knotenpunkten innerorts. Im Einführungsschreiben wird darauf hingewiesen, dass die StVO, VwV-StVO sowie die BOStrab im Falle von Unterschieden zu den RASt maßgeblich sind.

Zu den Nutzungsansprüchen an Straßenräume ${ }^{52}$ (Kapitel 4) können für Straßenbahnen folgende Angaben aus den RASt entnommen werden:

- Bild 13: maximale Fahrzeugbreite $W=2,65 \mathrm{~m}$

- der Bewegungsspielraum für Straßenbahnen ist mit 0,30 m angegeben. Der Verkehrsraum in Bögen verbreitert sich entsprechend (Bild 14, S. 26)

- Grundmaße für den Raumbedarf von Straßenbahnen ergeben sich aus den Verkehrsräumen (Fahrzeugabmessungen und Bewegungsspielräumen), den Sicherheitsräumen , den fahrzeugabhängigen Verbreiterungsmaßen bei Kurvenfahrt und den zusätzlichen Flächen für den Fahrgastwechsel bei Haltestellen.

In den RASt werden empfohlene Lösungen für typische Entwurfssituationen gegeben ${ }^{53}$. Bei der Auswahl einer typischen Entwurfssituation fließt das Vorhandensein einer Straßenbahn maßgeblich mit ein. Für folgende typische Entwurfssituationen gibt es Vorschläge einer Querschnittsaufteilung: Örtliche Geschäftsstraße, Hauptgeschäftsstraße, Verbindungsstraße, Anbaufreie Straße

Zu den möglichen Knotenpunktarten sind folgende Angaben enthalten ${ }^{54}$ :

- Die Rechts-vor-links-Regelung ist für Knotenpunkte mit Straßenbahnverkehr ausgeschlossen,

- Straßenbahnen auf besonderem Bahnkörper stehen dem Einsatz von Kreisverkehren nicht entgegen, wenn die signaltechnische Freischaltung gewährleistet ist.

- Ein Minikreisverkehr soll in der Regel bei Straßenbahnen nicht angelegt werden.

Die maßgeblichen Richtlinienvorgaben zu den Entwurfselementen der Strecke für Straßenbahnen sind folgende ${ }^{55}$ :

- Wenn auf dem anliegenden Fahrstreifen Straßenbahnen verkehren, sind Senkrechtparkstände zu vermeiden. Ansonsten sind breitere Zwischenstreifen erforderlich

- Generell ungeeignet für gemeinsame Führung des Radverkehrs mit Benutzungspflicht mit Fußgängern sind Straßen mit stark frequentierten Bus- oder Straßenbahnhaltestellen in Seitenlage ohne gesonderte Warteflächen.

\footnotetext{
[RASt 2006], S. 25ff

vgl. [RASt 2006], S. 33ff

[RASt 2006], S. 63ff

[RASt 2006], S. 69ff
} 
Die folgenden Inhalte sind zur Überquerung von Fahrbahnen durch Fußgänger gegeben ${ }^{56}$ :

- Lichtsignalanlagen sollten ununterbrochen (Tag und Nacht) in Betrieb gehalten werden. Eine Abweichung ist nur nach Prüfung eines sicheren Verkehrsablaufs möglich

- Überquerungsstellen an besonderen Bahnkörpern als Prinzipskizzen (Überquerungsstellen mit beidseitigen Aufstellflächen (i.d.R. als Z-Form) mit und ohne Signalisierung)

Wesentliche Richtlinienvorgaben für Straßenbahnen finden sich im Kapitel zu den Anlagen des Öffentlichen Personennahverkehrs ${ }^{57}$ :

- Die RASt verweist auf die BOStrab $\S 15$ Abs. 6 (Strecken sollen unabhängige oder besondere Bahnkörper haben) und ergänzt, dass ein Abweichen nur in begründeten Fällen möglich sei.

- Bei Straßen mit mehr als zwei Fahrstreifen je Richtung soll der Gleisbereich als besonderer Bahnkörper ausgebaut werden.

- In Einbahnstraßen mit gegenläufigem Straßenbahnverkehr soll das Gleis, das entgegen der Fahrtrichtung in der Straße verläuft, auf einen besonderen Bahnkörper verlegt werden.

- Ein eindeutig abgegrenzter ÖPNV-Fahrstreifen als besonderer Bahnkörper nach § 16 Abs. 6 BOStrab soll vorhanden sein, a) wenn Schnellverkehr der Straßenbahn (>50 km/h) vorgesehen ist, b) vor dem Anschluss von Rampen an Schienenstrecken in der 2. Ebene, c) wenn in Einbahnstraßen ein Zweirichtungsbetrieb der Straßenbahn durchgeführt wird.

- Nach Kapitel 6.1.10.2 (gemeinsamen Führung von Straßenbahn und Kfz-Verkehr) sollen Gleise ohne besonderem Bahnkörper in der Fahrbahnmitte liegen

- Aus Gründen der Verkehrssicherheit muss die Mitbenutzung von ÖPNV-Fahrstreifen mit Gleisen durch Radfahrer untersagt werden und durch andere geeignete Maßnahmen zur Sicherung des Radverkehrs ausgeschlossen werden.

- Es wird nach den RASt zwischen den Bahnkörpern in Tabelle 3-13 unterschieden:

Tabelle 3-13: Differenzierung der Bahnkörper nach den RASt

Quelle: $\quad$ [RASt 2006], S. 96

\begin{tabular}{|c|c|c|}
\hline \multicolumn{2}{|r|}{ Form } & Abgrenzung \\
\hline \multirow[t]{2}{*}{$\begin{array}{l}\text { Straßen- } \\
\text { bündiger } \\
\text { Bahn- } \\
\text { körper }\end{array}$} & $\begin{array}{l}\text { ohne räumliche Tren- } \\
\text { nung der Straßen- } \\
\text { bahn von den übrigen } \\
\text { Verkehrsarten }\end{array}$ & $\begin{array}{l}\text { Abgrenzung durch } \\
\text { Leitlinien (Zeichen } \\
340 \text { StVO) }\end{array}$ \\
\hline & $\begin{array}{l}\text { mit räumlicher Tren- } \\
\text { nung der Straßen- } \\
\text { bahn von den übrigen } \\
\text { Verkehrsarten }\end{array}$ & $\begin{array}{l}\text { Abgrenzung durch } \\
\text { Fahrstreifenbegren- } \\
\text { zungen (Zeichen } \\
295 \text { StVO) oder } \\
\text { Sperrflächen }\end{array}$ \\
\hline \multirow[t]{3}{*}{$\begin{array}{l}\text { Beson- } \\
\text { derer } \\
\text { Bahn- } \\
\text { körper }\end{array}$} & $\begin{array}{l}\text { mit geschlossenem } \\
\text { Oberbau* }^{\star} \\
\text { Ausführung in Beton, } \\
\text { Asphalt oder Pflaster }\end{array}$ & $\begin{array}{l}\text { Abgrenzung mit } \\
\text { Borden } \\
\text { Befahrbarkeit } \\
\text { durch Linienbusse } \\
\text { in Abstimmung } \\
\text { mit technischer } \\
\text { Aufsichtsbehörde } \\
\text { möglich }\end{array}$ \\
\hline & $\begin{array}{l}\text { mit geschottertem } \\
\text { Oberbau }\end{array}$ & \multirow[t]{2}{*}{$\begin{array}{l}\text { Abgrenzung in der } \\
\text { Regel mit Borden }\end{array}$} \\
\hline & $\begin{array}{l}\text { mit begrüntem Ober- } \\
\text { bau (Rasengleis) }\end{array}$ & \\
\hline
\end{tabular}


- In Kapitel 6.1.10.7 werden Aussagen zu Haltestellen für Straßenbahnen getroffen; es wird nach folgende Haltestellenformen unterschieden:

- Haltestellenkap

- Mittellage mit Seitenbahnsteigen

- Mittellage mit Mittelbahnsteigen

- angehobene Fahrbahn

- Zeitinseln (ggf. mit angehobener Fahrbahn)

- Zeitinseln dienen der Sicherung der Fahrgastwechsel, indem sie mit Hilfe von Lichtsignalanlagen die für den Fahrgastwechsel erforderliche Fahrbahnfläche kurzfristig frei halten. Bei Einfahrt in die Haltestelle sperrt ein Signal den allgemeinen Verkehr und verhindert die Nichtbeachtung des $\S 20$ StVO durch andere Verkehrsteilnehmer

Ergänzend zu den allgemeinen Knotenpunkarten werden für Knotenpunkte im Detail für Straßenbahnen folgende Vorgaben gegeben ${ }^{58}$ :

- Die Führung von Straßenbahnen über die Mitte der Kreisinsel von Kreisverkehren erfordert eine bedarfsgesteuerte signaltechnische Sicherung mit Sperrung aller Kreiszufahrten (Regellösung) oder der Kreisfahrbahn.

- Die Führung von Schienenverkehr über Minikreisverkehre ist nicht zulässig.

\begin{tabular}{|l|l|}
\hline Titel: & Richtlinien für Lichtsignalanlagen \\
\hline Veröffentlicht: & 2010, FGSV \\
\hline Quellenangabe: & [RiLSA 2010] \\
\hline
\end{tabular}

Die RiLSA enthalten grundlegende verkehrstechnische Bestimmungen und Empfehlungen für die Einrichtung und den Betrieb von Lichtsignalanlagen ${ }^{59}$

In Bezug zu Lichtsignalen und Signalfolgen werden mit Bezug zum Straßenbahnverkehr folgende Angaben gemacht ${ }^{60}$ :

- Zur Signalisierung von Straßen- und Stadtbahnen, die nach BOStrab verkehren, sollten die dort aufgeführten speziellen Lichtsignale verwendet werden:

- waagerechter Balken: Halt;

- senkrechter Balken: Frei ohne bedingt verträgliche Ströme;

Dreieck mit Spitze nach unten (Permissivsignal): Frei mit bedingt verträglichen Strömen;

- Punkt: Übergangszeit (Halt zu erwarten)

- Als Signalfolgen sind GESPERRT - FREI (bzw. PERMISSIV) - GESPERRT und GESPERRT FREI (bzW. PERMISSIV) - HALT ZU ERWARTEN - GESPERRT vorgesehen

- bei Querungen von Fahrbahnen des Individualverkehrs außerhalb von Knotenpunkten kann straßenseitig durch zweifeldige Signalgeber mit der Signalfolge DUNKEL - GELB - ROT - 
DUNKEL und für die ÖPNV-Fahrzeuge mit Signalen nach der BOStrab eine signaltechnische Sicherung erfolgen

Zur Signalprogrammstruktur können folgende Inhalte entnommen werden ${ }^{61}$ :

- eine signaltechnisch gesicherte Führung von Linksabbiegern ist umso dringlicher je mehr Konfliktfälle (z.B. mit Straßenbahnen) bestehen

- für abbiegende ÖPNV-Fahrzeuge ist in der Regel eine Sonderphase erforderlich

- Insbesondere bei starker Auslastung bedingt verträgliche Verkehrsströme können Aspekte der Verkehrssicherheit die Anwendung des Permissivsignals einschränken

- die Wartezeit bis zur Freigabe des Fußgängerverkehrs sollte möglichst kurz sein.

- an Fußgängerquerungsanlagen unabhängiger und besonderer Bahnkörper (§ 16 Abs. 6 und 7 BOStrab) ist eine Anforderungssteuerung durch das ÖPNV Fahrzeug sinnvoll; die Signalisierung der Fußgänger mit zweifeldigem gelbem Blinklicht scheint am besten geeignet.

- bei gesondert signalisierten Straßenbahnen soll das Übergangssignal in Abhängigkeit der zugelassenen Höchstgeschwindigkeit $\mathrm{V}_{\max }$ gezeigt werden

- das Übergangssignal kann entfallen, wenn am Signalstandort ausnahmslos zu halten ist; ein Signalwechsel von FREI auf GESPERRT innerhalb des Betriebsbremswegs ausgeschlossen wird und bei einer Höchstgeschwindigkeit von maximal $20 \mathrm{~km} / \mathrm{h}$

- Zwischenzeiten tz:

die pauschale Fahrzeuglänge $\mathrm{I}_{\mathrm{FZ}}$ einer Straßenbahn beträgt $15 \mathrm{~m}$

- die anzusetzende Einfahrgeschwindigkeit $\mathrm{V}_{\mathrm{e}}$ ist größer/ gleich $20 \mathrm{~km} / \mathrm{h}$

beim Konfliktfall „Straßenbahn räumt“ ist die reale Länge der Straßenbahnen zur Berechnung der Mindestfreigabezeit anzusetzen

Zu Signalisierung an Haltestellen werden in der RiLSA gesondert Angaben gemacht ${ }^{62}$ :

- Haltestelleninseln sind sinnvoll, wenn die übrige Fahrzeuge während Haltevorgang frei fließen sollen; bei signaltechnischer Sicherung der Querung zur Haltestelleninsel durch Furten ist es sicherheitsrelevant, dass bei einfahrenden ÖV-Fahrzeugen durch die Freigabe der Querung die Haltestelleninsel für Fahrgäste sicher erreicht werden kann

- dynamische Haltestellen (mit Zeitinsel) sollen bei Haltestellen in Mittellage und Wartefläche im Seitenraum Anwendung finden und können zusätzlich bei Haltestelleninseln Anwendung finden

- Die Signalgeber, die nur auf Anforderung geschaltet werden, sollten die Signalfolge DUNKEL GELB - ROT - DUNKEL zeigen

Mit Bezug zum Straßenbahnverkehr ist für Signalleuchten folgendes zu entnehmen ${ }^{63}$ :

- Gelten Kraftfahrzeugsignale nur für bestimmte Fahrtrichtungen, so ist dies durch gleiche Richtungspfeile in allen Leuchtfeldern des zugehörigen Signalgebers anzuzeigen. Dies gilt auch für Kombinationspfeile

- Hilfssignalgeber (gelbes Blinklicht ggf. mit Sinnbild) können bei Konfliktbereichen (z.B. Querungsanlage an Bahnkörpern) verwendet werden

- eine Sonderform des Hilfssignals ist das zweifeldige Springlicht (z.B. Querungsanlage an Bahnkörpern); der Warneffekt ist höher als bei einfachen Springlicht ${ }^{64}$

\footnotetext{
[RiLSA 2010], S. 13ff

[RiLSA 2010], S. 34f

[RiLSA 2010], S. 63ff

[RiLSA 2010], S. 66
} 
- Werden nicht alle Fahrstreifen einer Knotenpunktzufahrt gleichzeitig freigegeben, so sind für jede gesondert signalisierte Abbiegerichtung mindestens ein Signalgeber (mit Richtungspfeilen) und für die nicht abbiegende Fahrtrichtung mindestens zwei Signageber (in der Regel ohne Richtungspfeile) vorzusehen

- Wird mehrstreifig abgebogen, dann muss das Richtungssignal über der Fahrbahn wiederholt werden

Lichtsignalanlagen sollten ununterbrochen (Tag und Nacht) in Betrieb gehalten werden. Eine Abweichung ist nur nach Prüfung eines sicheren Verkehrsablaufs möglich ${ }^{65}$.

\begin{tabular}{|l|l|}
\hline Titel: & Empfehlungen für die Anlagen des ruhenden Verkehrs \\
\hline Veröffentlicht: & 2005, FGSV \\
\hline Quellenangabe: & [EAR 2005] \\
\hline
\end{tabular}

Die EAR behandelt die Planung, den Entwurf und den Betrieb von Anlagen für den ruhenden Verkehr innerhalb und außerhalb des öffentlichen Straßenraums.

Nach EAR ist beim Entwurf und Betrieb der Park-and-Ride-Anlagen die Führung von Straßenbahnen zu beachten: Die Zufahrtstrecken zu den Parkflächen und die Ausfahrten sind so zu legen, dass ein möglicher Stau nicht den Bus- oder Straßenbahnverkehr behindert ${ }^{66}$.

\begin{tabular}{|l|l|}
\hline Titel: & Empfehlungen für Radverkehrsanlagen \\
\hline Veröffentlicht: & 2010, FGSV \\
\hline Quellenangabe: & [ERA 2010] \\
\hline
\end{tabular}

Die ERA bilden die Grundlage für Planung, Entwurf und Betrieb von Radverkehrsanlagen. Sie gelten für den Neubau und die wesentliche Änderung von Straßen. Für bestehende Straßen wird ihre Anwendung empfohlen.

Im Rahmen der ERA werden im Kapitel Führungsformen des Radverkehrs an innerörtlichen Hauptverkehrsstraßen explizite Empfehlungen für den Radverkehr auf Fahrbahnen mit Straßenbahnen (unter Beachtung der BOStrab) sowie an Straßenbahnhaltestellen gegeben. Eine Führung des Radverkehrs im Seitenraum wird durch die Konflikte mit dem Schienenverkehr weitgehend ausgeschlossen ${ }^{67}$. Wo eine solche Führung nicht umsetzbar ist, in Abhängigkeit von den verkehrlichen Rahmenbedingungen, die Führung rechts von den Gleisen entsprechend der Einsatzbedingungen und Entwurfshinweise gemäß der Tabelle 3-14 zu prüfen.

\footnotetext{
[RiLSA 2010], S. 73

[EAR 2005], S. 65

[ERA 2010], S. 30
} 
Tabelle 3-14: Regellösungen bei Radverkehr auf Fahrbahnen mit Straßenbahn Quelle: [ERA 2010], S. 31

\begin{tabular}{|c|c|c|}
\hline Führungsform & Einsatzbedingungen & Entwurfshinweise \\
\hline $\begin{array}{l}\text { rechts von den Gleisen außerhalb des Stra- } \\
\text { Benbahn-Verkehrsraums mit Überholmög- } \\
\text { lichkeit im Radverkehr in diesem Bereich } \\
\text { Verkehrsraum Straßenbahn } \\
\end{array}$ & \multirow{2}{*}{$\begin{array}{l}\text { - Abstand von Straßenbahn- } \\
\text { Verkehrsraum zu Bord } \geq 1,60 \mathrm{~m} \\
\text { bzw. zu Parkstreifen } \geq 2,10 \mathrm{~m} \\
\text { (zur Erhöhung der Sicherheits- } \\
\text { abstände möglichst mehr, sofern } \\
\text { entsprechende Flächen vorhanden) } \\
\text { - } \mathrm{V}_{\text {zul }} \text { bis } 50 \mathrm{~km} / \mathrm{h} \\
\text { - kein oder geringer Lieferverkehr }\end{array}$} & \multirow{2}{*}{$\begin{array}{l}\text { - bei Notwendigkeit entsprechend } \\
\text { dem Abschnitt 2.3.3 Radfahrstrei- } \\
\text { fen oder Schutzstreifen realisieren } \\
\text { - wenn kein Schutzstreifen oder } \\
\text { Radfahrstreifen: Kennzeichnung } \\
\text { der Grenze zum Straßenbahn- } \\
\text { Verkehrsraum sinnvoll } \\
\text { (z. B. Materialwechsel) } \\
\text { - ab 2,00 m Breite neben Bord und } \\
\text { großem Parkdruck Überwachung, } \\
\text { um unerlaubtes Parken zu verhin- } \\
\text { dern }\end{array}$} \\
\hline Rad …는 & & \\
\hline $\begin{array}{l}\text { rechts von den Gleisen außerhalb des Stra- } \\
\text { Benbahn-Verkehrsraums ohne Überhol- } \\
\text { möglichkeit im Radverkehr in diesem } \\
\text { Bereich }\end{array}$ & \multirow{2}{*}{$\begin{array}{l}\text { - Abstand von Straßenbahn- } \\
\text { Verkehrsraum zu Bord möglichst } \\
\geq 1,30 \mathrm{~m} \text { bzw. zu Parkstreifen } \\
\geq 1,80 \mathrm{~m} \\
\text { - } \mathrm{V}_{\text {zul }} \text { bis } 50 \mathrm{~km} / \mathrm{h} \\
\text { - kein oder geringer Lieferverkehr } \\
\text { - wenig Überholbedarf im Radver- } \\
\text { kehr aufgrund geringer Radver- } \\
\text { kehrsstärke (das heißt nur seltene } \\
\text { Überquerungen des Gleisbereiches } \\
\text { durch den Radverkehr) }\end{array}$} & \multirow[t]{2}{*}{$\begin{array}{l}\text { - Kennzeichnung der Grenze zum } \\
\text { Straßenbahn-Verkehrsraum sinnvoll } \\
\text { (z. B. Materialwechsel) } \\
\text { - Beschränkung auf kurze Strecken- } \\
\text { abschnitte sinnvoll }\end{array}$} \\
\hline Rad $\ldots \ldots$ & & \\
\hline
\end{tabular}

Bei baulichen Zwangslagen, die eine Seitenraumführung oder die in der Tabelle 3-14 dargestellten Führungsformen nicht ermöglichen, kommen im Einzelfall auch die in der Tabelle 3-15 dargestellten Möglichkeiten in Frage. Sie sollten möglichst auf kurze Abschnitte beschränkt werden oder regelmäßig Ausweichstellen vorsehen, an denen der Radverkehr eine Straßenbahn überholen lassen kann.

Tabelle 3-15: Sonderlösungen bei Radverkehr auf Fahrbahnen mit Straßenbahn Quelle: [ERA 2010], S. 31

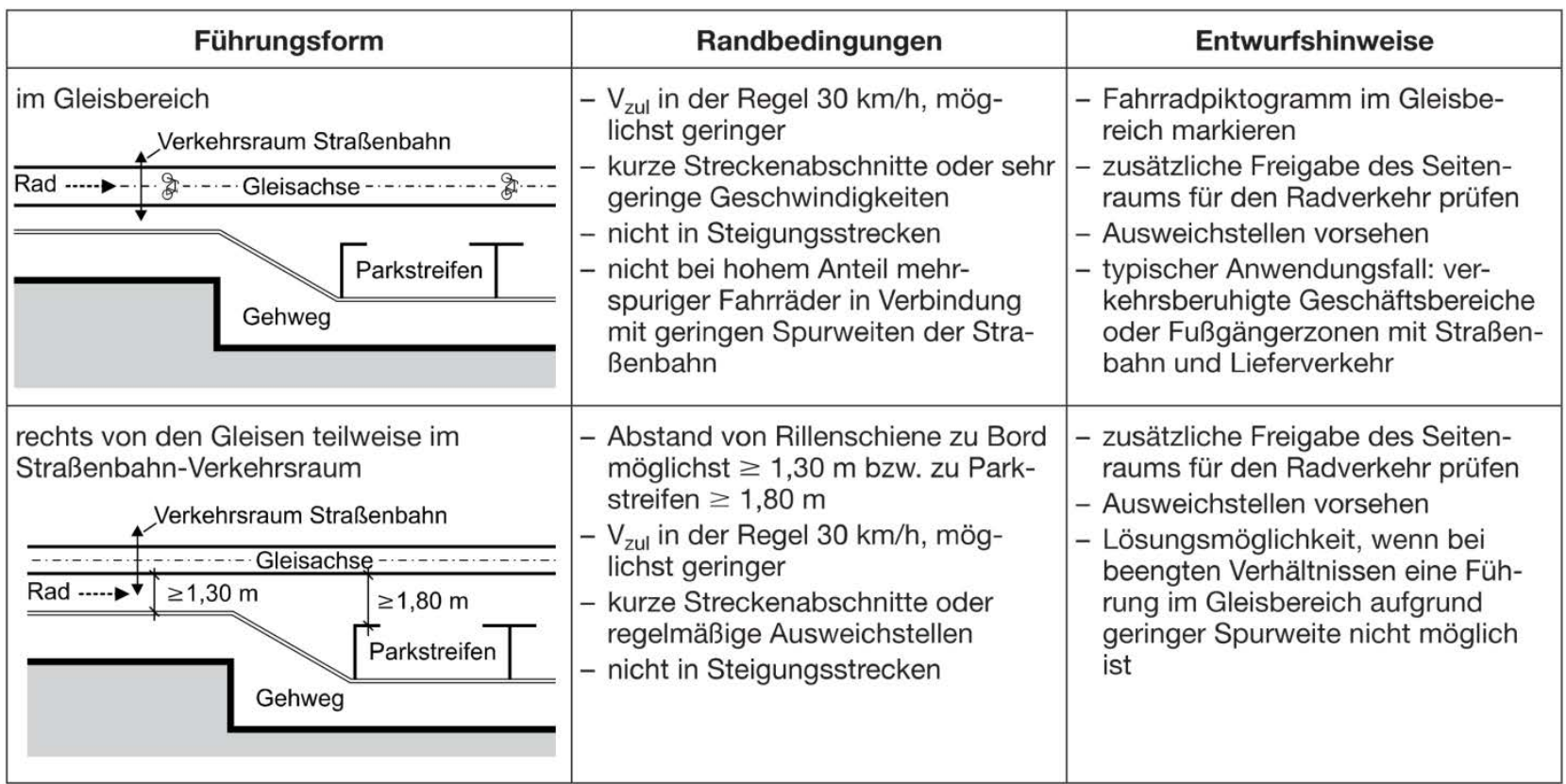


Bei der Führung des Radverkehrs ist die spitzwinklige Überquerung von Straßenbahngleisen zu vermeiden $^{68}$. Ab einem Winkel von 50 gon oder mehr ist die Überquerung von Rillenschienen problemlos möglich. Wo Überquerungen der Gleise obligatorisch sind, z. B. vor Haltestellenkaps, können Überquerungsstelle und Überquerungswinkel durch Markierungen verdeutlicht werden.

Straßenbahnhaltestellen in Mittellage mit Bahnsteigen stellen keine besonderen Anforderungen an die Radverkehrsführungen dar ${ }^{69}$. Bei Straßenbahnhaltestellen in Seitenlage ist dagegen die Wahl der Haltestellenform von vielfältigen Einflussfaktoren abhängig. Mögliche Kombinationen von Radverkehrsführung und Haltestellenform zeigen die Tabelle 3-16. Besonders zu berücksichtigen ist die Konfliktlage zwischen Radverkehr und ein- bzw. aussteigenden oder wartenden Fahrgästen bei einer Radverkehrsführung im Seitenraum.

Tabelle 3-16: Kombinationen von Radverkehrsführung und Haltestellenform bei Straßenbahnhaltestellen in Seitenlage Quelle: $\quad$ [ERA 2010], S. 34

\begin{tabular}{|l|l|l|l|}
\hline $\begin{array}{l}\text { Rad- } \\
\text { verkehrs- } \\
\text { führung }\end{array}$ & Haltestellenform auf der Fahrbahn & Angehobene Fahrbahn & \multicolumn{1}{c|}{ Haltestellenkap } \\
\hline $\begin{array}{l}\text { Mischverkehr auf der } \\
\text { Fahrbahn/Radfahrstreifen/ } \\
\text { Schutzstreifen }\end{array}$ & gut geeignet & gut geeignet & $\begin{array}{l}\text { bedingt geeignet (bei Rad- } \\
\text { verkehr rechts der Gleise: } \\
\text { angehobene Radverkehrs- } \\
\text { führung }\end{array}$ \\
\hline Führungen im Seitenraum & $\begin{array}{l}\text { bedingt geeignet (abhängig } \\
\text { von Seitenraumbreiten) }\end{array}$ & $\begin{array}{l}\text { bedingt geeignet (abhängig } \\
\text { von Seitenraumbreiten) }\end{array}$ & gut geeignet \\
\hline
\end{tabular}

Bei einer Seitenraumführung des Radverkehrs auf der Strecke bieten Haltestellenkaps in der Regel ausreichend zusätzliche Flächen für Warten und Ein- und Aussteigen der Fahrgäste ${ }^{70}$. Die Radverkehrsführung kann dann möglichst geradlinig beibehalten werden. Eine Streckenführung des Radverkehrs im Gleisbereich wird am Haltestellenkap beibehalten.

Plangleiche Überquerungsstellen an Strecken der Eisen-, Straßen- oder Stadtbahn stellen besondere Anforderungen an die Radverkehrsführung, $\mathrm{da}^{71}$

- sehr große Sichtfelder zu gewährleisten sind,

- technische Sicherungsanlagen das zügige Räumen des Schienenweges nicht verhindern dürfen und

- aus Sicherheitsgründen Gleisüberquerungen im spitzen Winkel (<50 gon) vermieden werden sollten.

Bei Führung des Radverkehrs auf der Fahrbahn werden die dort bestehenden

Sicherungseinrichtungen zur Gleisquerung genutzt.

Besondere bzw. unabhängige Bahnkörper können an Überquerungsstellen durch Signalisierung mittels gelbem Springlicht (zwei abwechselnd aufleuchtende Leuchtfelder bei Annäherung einer Bahn) gesichert werden ${ }^{72}$. Alternativ ist eine signaltechnische Sicherung mit der Grundstellung Dunkel für den Fußgänger- und Radverkehr und Halt für Nahverkehrsfahrzeuge möglich, die bei Schienenverkehr Rot für den nichtmotorisierten Verkehr zeigt.

\footnotetext{
8 [ERA 2010], S. 30

9 [ERA 2010], S. 34

[ERA 2010], S. 35

[ERA 2010], S. 35

2 [ERA 2010], S. 36
} 
Wegen ihrer Konfliktträchtigkeit ist zu vermeiden Radverkehrsanlagen im engeren Knotenpunktbereich enden lassen ${ }^{73}$.

\begin{tabular}{|l|l|}
\hline Titel: & Empfehlungen für Fußgängeranlagen \\
\hline Veröffentlicht: & 2002, FGSV \\
\hline Quellenangabe: & [EFA 2002] \\
\hline
\end{tabular}

Die Empfehlungen befassen sich mit den Anforderungen des Fußgängerverkehrs im Zusammenhang mit der Planung von Verkehrsanlagen.

Generell ungeeignet für gemeinsame Führungen von Fuß-und Radverkehr sind Straßen mit stärker frequentierten Bus- oder Straßenbahnhaltestellen ${ }^{74}$.

Die Sperrung des Gleisbereiches für querende Fußgänger durch Rot oder die Warnung vor der Straßenbahn durch Blinklicht sollte stets auf den Zeitbereich des Herannahens der Straßenbahn und Vorbeifahrens beschränkt bleiben ${ }^{75}$.

nach EFA kann nach folgenden Haltestellenformen differenziert werden ${ }^{76}$ :

- Haltestelle in Mittellage

- mit Mittelinsel

- mit Ausstieg auf die Fahrbahn

- Haltestelle in Fahrbahnseitenlage

- am Fahrbahnrand

- Haltestellenkap

Zu Haltestellen des ÖPNV werden folgende Aussagen getroffen ${ }^{77}$ :

- bei Neuanlage von Haltestellen sollte aus Akzeptanzgründen ein niveaugleicher Zugang realisiert werden,

- Haltestelleninseln sollten an beiden Inselenden zu jeder Straßenseite eine Querungsanlage haben,

- wichtig ist die Gewährleistung des Haltestellenzugangs unmittelbar vor dem Eintreffen des Linienfahrzeuges und während eines Großteils der Fahrgastwechselzeit,

- In Bereichen mit stark frequentierten Seitenräumen kann der Zugang zur Haltestelle oder zum Linienfahrzeug auf der gesamten Haltestellenlänge möglich sein. Eine wirksame Sicherung der Fahrgäste lässt sich in diesen Fällen durch Anordnung einer lichtsignaltechnisch gesicherten dynamischen Haltestelle erreichen,

- Erfolgt bei einer Haltestelle vor dem Knotenpunkt mit LSA bei einlaufender und haltender Straßenbahn eine Freigabezeitverlängerung für die Straßenbahn, so darf der parallel geführte Kfz-Verkehr nicht gleichzeitig Grün bzw. eine Grünzeitverlängerung erhalten, damit die Straßenbahn von beiden Straßenseiten ungefährdet erreicht werden kann.

- Fußgängerüberwege dürfen nicht über Straßen mit Straßenbahnen ohne eigenen Gleiskörper angelegt werden (Verweis auf R-FGÜ 2001) ${ }^{78}$ 


\begin{tabular}{|l|l|}
\hline Titel: & Empfehlungen für Anlagen des öffentlichen Personennahverkehrs \\
\hline Veröffentlicht: & 2013, FGSV \\
\hline Quellenangabe: & [EAÖ 2013] \\
\hline
\end{tabular}

Die EAÖ 2013 behandelt den Entwurf und die Dimensionierung der technischen Anlagen des ÖPNV auf den überregionalen, regionalen und nah-/kleinräumigen Verbindungsstufen entsprechend den RIN und basiert auf dem PBefG, der BOStrab und den RASt $2006^{79}$, ergänzt die RASt 2006 sowie u.a. die ESG 2011, RiLSA 2010 und H VÖ 2009 um ÖPNV-spezifische Aspekte ${ }^{80}$.

Straßenbahnen werden in Anlehnung an die RASt nur für örtliche Geschäftsstraßen, Hauptgeschäftsstraßen, anbaufreie Straßen und Verbindungsstraßen vorgesehen ${ }^{81}$.

Zu den Grundmaßen und technischen Vorschriften werden in den EAÖ 2013 umfangreiche Erläuterungen gegeben ${ }^{82}$ :

- Straßenbahnen sind Schienenbahnen auf einem straßenbündigen/ straßenabhängigen Bahnkörper oder einem besonderen Bahnkörper

- es wird der Hinweis darauf gegeben, dass der Entwurf der Fortschreibung der BOStrab (Stand Aug. 2013) nicht mehr zwischen besonderem und unabhängigem Bahnkörper unterscheidet

- besondere Bahnkörper liegen im Verkehrsraum öffentlicher Straßen, sind jedoch vom übrigen Verkehr durch Bordsteine, Leitplanken, Hecken, Baumreihen oder andere ortsfeste Hindernisse getrennt

- für die EAÖ maßgebend ist das PBefG und die BOStrab sowie nachgeordnet der BOStrab u.a. die technischen Regelwerke BOStrab-Trassierungsrichtlinien, BOStrab-Lichtraum-Richtlinien, Technische Regeln Spurführung und Oberbau-Richtlinien und Oberbau-Zusatzrichtlinien für Bahnen nach BOStrab

- mit Bezug zur BOStrab § 34 gelten für Fahrzeuge und Züge folgende Grundmaße:

Breite 2,65 m bis Höhe 3,40 m (2,25 m ab Höhe 3,40 m),

- Höhe: 4,00 m

- Länge: $75,00 \mathrm{~m}$

- eine Unterscheidung der Bahnkörper erfolgt nach straßenbündigen Bahnkörper und besonderen Bahnkörper (Bezug zur BOStrab Entwurf $2013 \S 16$ ):

- straßenbündige Bahnkörper sollen in der Mitte der Fahrbahn liegen (Bezug zur RASt 2006)

- besondere Bahnkörper können in Mittel- oder Seitenlage angeordnet sein

- Beim Lichtraum von Straßenbahnfahrzeugen erfolgt ein Verweis nahezu ausschließlich auf die BOStrab bzw. die BOStrab-Lichtraumrichtlinien

- Bei den Sicherheitsräumen Sicherheitsräume erfolgt ein Verweis nahezu ausschließlich auf die BOStrab $\S 19$ und VBG-Fachinformation, BGI 5040 83

- Bei den Elementen der Gleistrassierung erfolgt ein Verweis nahezu ausschließlich auf die BOStrab-Trassierungsrichtlinien

\footnotetext{
[EFA 2002], S. 35 (Anhang R-FGÜ 2001)

[EAÖ 2013], S. 13

[EAÖ 2013], S. $14 f$

[EAÖ 2013], S. 22 und Bild 10, S. 23

[EAÖ 2013], S. 24ff

[VBG 2011]
} 
- die Geschwindigkeit bei straßenbündigen Bahnkörper ist i.d.R. 50 km/h bzw. die Geschwindigkeit des übrigen Verkehrs; bei besonderem Bahnkörper i.d.R. 70 km/h (Bezug zu BOStrab § 50)

- Haltestellen sollen als solche kenntlich sein (Doppelhaltestellen gesondert), Bahnsteige besitzen; die Zu und Abgänge müssen sicher und bequem sein (Bezug zur BOStrab § 31)

- entlang der Bahnsteigkante soll eine nutzbare Breite von mindestens 2,50 m vorhanden sein (BOStrab 1987: 2,00 m (1,50 m) )

- Zwischen festen einbauten und der Bahnsteigkante müssen mindestens 1,50 m Abstand sein und bei Haltestelleinsel zusätzlich 0,50 m Sicherheitsraum zur Fahrbahn hin.

Bezüglich Entwurf und Gestaltung von Straßenbahnverkehrsanlagen werden in den EAÖ 2013 strukturiert in Kapiteln nach ÖPNV-Fahrwegen, Knotenpunkten, Haltestellen, Überquerungsstellen und weiteren Betriebsanlagen und Einrichtungen differenziert Angaben gemacht, deren sicherheitsrelevante Inhalte im Folgenden dargestellt werden sollen:

Entwurf und Gestaltung von ÖPNV-Fahrwegen ${ }^{84}$ :

- ÖPNV-Fahrstreifen in Mittellage:

- ein relativ großer Flächenbedarf ist notwendig

- Einschränkungen für Linksabbiegende Kfz zwischen den Knotenpunkten (Grundstückszufahrten)

- keine Einschränkungen durch widerrechtlich haltende Kfz

- ÖPNV-Fahrstreifen in Seitenlage:

- ggf. günstigere Erreichbarkeit für Fahrgäste

- Grundstücksanbindung müssen ggf. zusätzlich technisch gesichert sein

- der Übergangsbereich von Mittellage zu Seitenlage sollte nur mit Signalschutz erfolgen

- Anfang und Ende sowie jede Unterbrechung von ÖPNV-Fahrstreifen sind klar erkennbar auszubilden

- Besondere Bahnkörper müssen durch ortsfeste Hindernisse (z. B. Borde) abgegrenzt sein

- nach BOStrab § 16 (6) nehmen durch ortsfest abgegrenzte ÖPNV-Fahrstreifen Straßenbahnen nicht am Straßenverkehr teil; damit ist eine höhere zulässige Höchstgeschwindigkeit für Straßenbahnen bei baulich abgegrenzten Fahrstreifen möglich

- nach folgenden Bahnkörpern und Bauformen wird in der EAÖ 2013 unterschieden: 
Tabelle 3-17: Bahnkörper und Bauformen

Quelle:

[EAÖ 2013], S. 40

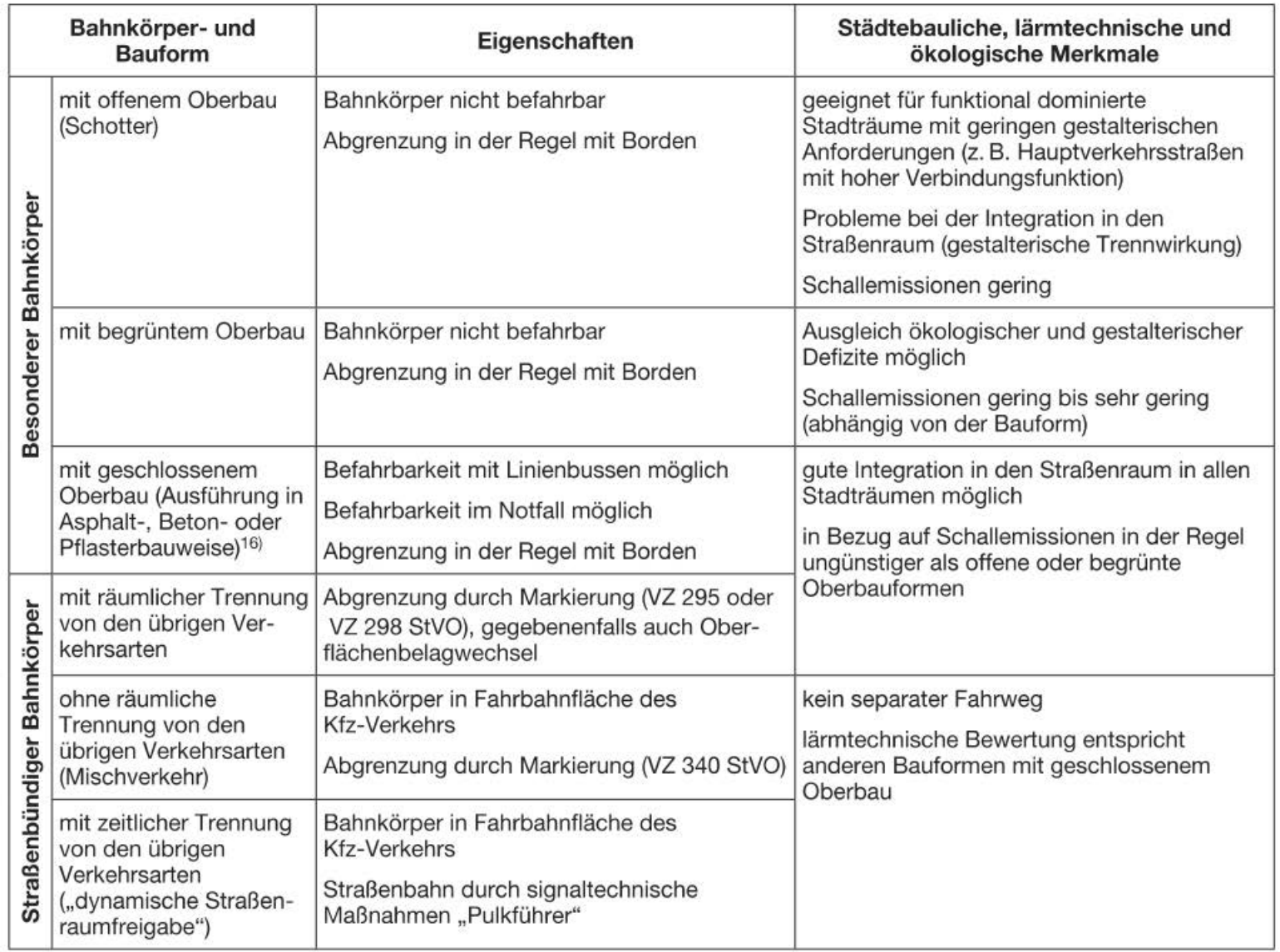

- auf Straßen mit straßenbündigem Bahnkörper sollte der Radverkehr außerhalb des Verkehrsraums der Straßenbahn geführt werden

- im Haltestellenbereich ist bei vorgezogenen Borden eine Führungsform zu wählen, die spitzwinkliges Queren durch Radfahrer vermeidet

Knotenpunkte ${ }^{85}$ :

- Knotenpunkte werden nach den Vorgaben der RASt 2006 angelegt. Die RASt fordern allgemein, dass Knotenpunkte rechtzeitig erkennbar, begreifbar, übersichtlich sowie gut und sicher befahrbar bzw. begehbar sein müssen.

- Wendefahrbahnen (können als Ersatz für Linksabbiegestreifen vorgesehen werden; Bei Querung von besonderen Bahnkörpern ist eine Lichtsignalanlage notwendig

- Knotenpunkte mit rechts-vor-Link-Regelung ist in Knotenpunkten mit Straßenbahnverkehr ausgeschlossen

- Bei nicht signalgeregelten Knotenpunkten sollten die Straßen mit Linienverkehr als Vorfahrtsstraßen ausgewiesen werden (Bezug zu § 8 VwV-StVO)

- An Kreisverkehren wird bei der Führung einer Straßenbahn über die Kreisinsel eine Signalisierung mit Sperrung der Kreisfahrbahn (gängige Lösung) oder aller Kreiszufahrten notwendig.

- eine Führung von Straßenbahnen über Minikreisverkehre ist nicht zulässig (vgl. RASt 2006) 
Bahnübergänge werden im Kapitel Lichtsignalgeregelte Knotenpunkte ${ }^{86}$ mit Verweis auf die BOStrab thematisiert und sollen im Folgenden gesondert vorgestellt werden:

- Straßenbahnen haben an Bahnübergängen grundsätzlich Vorrang (Andreaskreuz) o. eine andere örtliche Regelung besteht (z.B. Lichtsignalanlage)

- Bahnübergänge mit Vorrang für die Straßenbahn müssen

- durch Übersicht auf die Bahnstrecke gesichert sein

- technisch gesichert sein, wenn sie innerhalb eines Tages in der Regel von mehr als 100 Kraftfahrzeugen überquert werden, auf der kreuzenden Straße schneller als $50 \mathrm{~km} / \mathrm{h}$ gefahren werden darf oder auf dem Bahnübergang Straßenbahnen auf Zugsicherung fahren und

als Bahnübergänge von Geh- und Radwegen welche a) außerhalb des Bereiches von Haltestellen und Straßenkreuzungen liegen oder b) die Gleisanlagen im Bereich von Haltestellen oder Straßenkreuzungen nicht ungefähr rechtwinklig kreuzen und nicht technisch gesichert sind, zusätzlich mit Umlaufsperren oder ähnlich wirkenden Einrichtungen ausgerüstet oder durch Lichtzeichen signalisiert sein

- Die technische Sicherung eines Bahnüberganges erfordert

- Geber für Lichtzeichen mit der Farbfolge Gelb - Rot nach BOStrab, welche mit Halbschranken verbunden sein können und

- Geber für Überwachungssignal Bü 1 (Bahnübergang darf von Schienenfahrzeug befahren werden) im Anhaltewegabstand vor dem Bahnübergang oder eine Zugsicherungsanlage.

Bei Strecken mit Fahren auf Sicht auf denen ausschließlich straßenabhängige Bahnen verkehren, dürfen anstatt der Bü-Signale auch Fahrsignale unmittelbar vor dem Bahnübergang verwendet werden

Haltestellen ${ }^{87}$ :

- Rechtliche Vorgaben setzen das PBefG, die BOStrab sowie in Teilen auch das AEG ${ }^{88}$ und die EBO

- Anforderungen der Fahrgäste sind u.a. die sichere, barrierefreie und komfortable Erreichbarkeit der Haltestellen

- Die Anforderungen des Betreibers sind u.a. ein sicherer Fahrgastwechsel, die zügige, sichere und störungsfreie Ein- und Ausfahrt der Nahverkehrsfahrzeuge, gegebenenfalls unterstützt durch verkehrsregelnde Maßnahmen

- Die Anforderungen der anderen Straßennutzer sind eine übersichtliche Positionierung der Haltestelle und der Zugänge, die gute Einsehbarkeit von Überquerungsstellen und Sicht auf eventuelle Laufeinsteiger, die gute Einsehbarkeit von abbiegenden und einfädelnden Nahverkehrsfahrzeugen, die Minimierung von Rückstau hinter den auf der Fahrbahn haltenden Nahverkehrsfahrzeugen und die möglichst sichere, umwegfreie und für andere Verkehrsteilnehmer gut einsehbare Führung des Radverkehrs

- die Anforderungen des Fußgängerverkehrs sind ausreichende Geh-, Verweil- und Warteflächen im Seitenbereich, insbesondere an Knoten und Haltestellenzugängen

- eine Differenzierung der Haltestellen erfolgt nach

- Lage im Straßenquerschnitt: Seitenraum oder Insellage 
- Bauform: Haltestellenkap, Seitenbahnsteig (Haltestelle am Fahrbahnrand mit oder ohne Fahrbahnanhebung), Mittelbahnsteig

- Haltestellen im Seitenraum

- es besteht ein Konfliktpotenzial zwischen Radfahrern, Fußgängern sowie Ein- und Aussteigern

- daher ist eine ausreichende Bemessung des Seitenraums erforderlich (Bezug zu RASt und EFA)

- bei spitzwinkliger Kreuzung von Schienen und Mischverkehrsfläche (Kfz/ Rad) ist die ERA zu beachten

- bei hohem Verkehrsaufkommen oder schlechten Sichtverhältnissen sind folgende Querungshilfen möglich:

- Anordnung der Haltestelle in der Nähe von LSA-gesicherten Querungen

- Punktuelle Querungshilfen in Fahrbahnmitte hinter dem stehenden ÖPNV-Fahrzeug (mit versetzten Richtungshaltestellen)

- Langgezogene Mittelinsel (mit gegenüberliegenden Richtungshaltestellen)

- Fahrbahnverengung in Form direkt gegenüberliegende Haltestellenkaps

- Wird der Radverkehr auf der Fahrbahn mit Radfahrstreifen oder Schutzstreifen geführt, werden diese im Bereich der Haltestelle mittels Zeichen 299 StVO (Grenzmarkierung für Halt- und Parkverbote) unterbrochen

- Prinzipskizzen zu Fahrbahnrand- , Kaphaltestelle, Haltestelle mit Fahrbahnanhebung und Haltestellen in Insellage sind aufgeführt ${ }^{89}$

- die Haltestellenform 'Mittellage, Wartefläche und Haltefläche getrennt' sieht die EAÖ 2013 nur in Verbindung mit einer angehobenen Fahrbahn vor. In diesem Fall gilt:

- beim Halt der Straßenbahn muss der parallele IV gestoppt werden, um den Fahrgastwechsel abzusichern. (Zeitinsel)

- Haltestellen in Insellage

- es besteht ein Konfliktpotenzial durch Querungsvorgänge von ein- und aussteigenden Fahrgästen über parallele Fahrstreifen des MIV

Überquerungsstellen ${ }^{90}$ :

- die Ausführungen von Querungsstellen soll gemäß RASt und EFA durchgeführt werden

- Fußgängerüberwege bei straßenbündigen Bahnkörpern sind unzulässig

- zwischen besonderen Bahnkörpern und der Fahrbahn ist nach BOStrab eine Aufstellfläche anzuordnen (Regellösung) oder eine Signalisierung (Querung in einem Zug) notwendig

- nach ERA 2013 erfolgt eine Differenzierung von Überquerungsstellen nach folgenden Bauformen:

-Z-Führung/ Führung im Versatz (mit/ ohne Signalisierung)

- gradlinige Führung (mit/ ohne Signalisierung)

- nach ERA 2013 erfolgt folgende Differenzierung von Überquerungsstellen nach Lage im Netz

- an Knotenpunkten häufig in Verbindung mit einer Haltestelle

- an Streckenabschnitten mit Haltestelle

89 [EAÖ 2013], S. 64, S. 66, S. 68, S. 70, S. 72

90 [EAÖ 2013], S. 85ff 
an Streckenabschnitten ohne Haltestelle.

- die wichtigsten Voraussetzungen um auf eine Signalisierung zu verzichten sind:

- die Gleisquerung befindet sich außerhalb von signalisierten Knotenpunkten.

- gute Erkennbarkeit und Einsehbarkeit

- „Z-Form“ als Grundform

- großzügig Dimensionierung (Kinderwagen, Fahrradanhänger, Rollstühle)

- die Z-Querung bzw. Querung mit Versatz hat sich als sichere Form der Querung bewährt

- nachteilig bei der gradlinigen Führung ist, dass der Blick der Fußgänger nicht mehr auf die entgegenkommende Bahn gerichtet ist und dadurch diese Variante im Allgemeinen als weniger sicher gilt,

- bei Anordnung in Mittellage und gleichzeitig vorhandener Signalisierung sowohl der Gleis- als auch der Fahrbahnquerung, kann es bei unterschiedlicher Signalstellung (z.B.

Fahrbahnquerung für den Fußgänger frei, Gleisquerung hingegen gesperrt) zu Fehldeutungen kommen,

- zu beachten bei Überquerungsstellen für den Radverkehr sind:

- sehr große Sichtfelder

- Ausgestaltung der technischen Sicherungsanlagen so, dass das zügige Räumen des Gleisbereiches gewährleistet ist

- Gleisquerungen im spitzen Winkel (< 50 gon) sind zu vermeiden

Q Querungsstellen für den Radverkehr außerhalb von Knotenpunkten sind zu signalisieren oder in Z-Form auszuführen

- bei der Führung des Radverkehrs gemeinsam auf oder parallel zur Fahrbahn werden die Sicherheitseinrichtungen der Straße (gegebenenfalls ergänzt durch Signalgeber für den Radverkehr) genutzt

- bei Überquerungsstellen für Fußgänger ist folgendes zu beachten:

- bei der Signalisierung ist aufgrund der Barrierefreiheit das Zwei-Sinne-Prinzip zu beachten

- signaltechnische Sicherung erfolgt vorzugsweise durch eine ROT-DUNKEL-Schaltung oder durch ein Warnlicht (gelbes Springlicht)

- Bei Gelbblinkern kann keine Freigabe der Querung durch das Fahrsignal F1 erfolgen; zur Unterstützung des Fahrpersonals (Information: Anlage ist in Betrieb) kann hier lediglich das Signal F0 (Halt)-Dunkel vorgesehen werden

- Eine eindeutige Signalisierung ist mittels ROT-DUNKEL-Schaltung in Verbindung mit den Signalbildern F0 (Halt) und F1 (Fahrt freigegeben) möglich, weshalb diese Variante vorzuziehen ist

- grundsätzlich ist eine einheitliche Lösung im gesamten Stadtgebiet günstig

- bei Überquerungsstellen an Knotenpunkten werden folgende Inhalte gegeben:

- Ist am Knotenpunkt eine Straßenbahnhaltestelle angeordnet, so sind an beiden Bahnsteigenden Zugänge vorzusehen

- bei einer Signalisierung ist dem sicheren Zugang zur Haltestelle Vorrang gegenüber einer ungestörten „Grünen Welle“ des Kfz-Verkehrs zu geben

an Knotenpunkten ohne Signalisierung ist der Straßenzug mit Straßenbahn als Vorfahrtstraße auszubilden 
- an Überquerungsstellen an Strecken mit Haltestellen sind folgende sicherheitsrelevante Vorgaben zu beachten:

- Grundsätzlich gelten die gleichen Gestaltungsgrundsätze wie an Knotenpunkten

- Unterschiede ergeben sich bei der Signalisierung:

- Mit einer Signalisierung ist grundsätzlich kein Sicherheitsgewinn verbunden

- Vielmehr ist Gestaltung wichtig (Übersichtlichkeit, Erkennbarkeit)

- Überquerungsstellen an Strecken ohne Haltestellen sind erforderlich, wo wichtige Gehwegverbindungen existieren:

- eine Abwägung bei der Anzahl der Querungen muss erfolgen um "wildes Queren" zu minimieren

- Absperrung zwischen den Überquerungsstellen nach BOStrab § 16 (6) z.B. durch Hecken kann sinnvoll sein

- Überquerungsstellen an Strecken ohne Haltestellen sollten als Z-Querung ausgeführt und gegebenenfalls durch Lichtsignale gesichert sin (insbesondere bei höheren Geschwindigkeiten)

Schilder und Signale:

- Zusätzlich zur Beschilderung gemäß StVO sind Schilder und Signale nach BOStrab erforderlich

- Im Bereich von Gleisen werden diese im Regelfall rechts neben dem zugehörigen Gleis im Lichtraum-Abstand aufgestellt 


\section{4 Sicherheit von Straßenbahnen im Vergleich zu anderen ausgewählten innerstädtischen Verkehrsmitteln: bundesweite Analyse}

Ziel der vergleichenden Beurteilung der Verkehrssicherheit ist im Wesentlichen zu untersuchen, wie sich die Unfallzahlen und deren Schwere zwischen den motorisierten Verkehrsmitteln PKW, Bus und Straßenbahn unterscheiden.

Um Unfallzahl und Unfallschwere zusammenzufassen und vergleichend gegenüberstellen zu können, werden üblicherweise Unfallkosten berechnet, die sich aus den volkswirtschaftlichen Verlusten durch die Straßenverkehrsunfälle ergeben.

Neben den absoluten Unfallzahlen unterschiedlicher Unfallschwerekategorien bzw. deren berechneten Unfallkosten ist es darüber hinaus erforderlich aussagekräftige Unfallkenngrößen miteinander zu vergleichen:

- Anzahl der Unfälle in Abhängigkeit der Fahrleistung (Unfallrate) sowie die Unfallkosten in Abhängigkeit der Fahrleistung (Unfallkostenrate), d.h. die Anzahl der Unfälle bzw. Höhe der Unfallkosten die beispielsweise bei einer Fahrleistung von 1.000 Fahrzeugkilometer auf einem Straßenabschnitt entstanden sind,

- Anzahl der Unfälle in Abhängigkeit der Beförderungsleistung sowie die Unfallkosten in Abhängigkeit der Beförderungsleistung, d.h. die Anzahl der Unfälle bzw. Höhe der Unfallkosten die beispielsweise bei einer Beförderungsleistung von 1.000 Personenkilometern auf einem Straßenabschnitt entstanden sind.

Als Datenbasis der zu vergleichenden Unfälle wurden für die Modi PKW und Bus die Daten der Straßenverkehrsunfallstatistik des Statistischen Bundesamtes von 2009 bis 2011 mit Beteiligung des jeweiligen Verkehrsmittels innerhalb von Ortschaften für Unfälle mit Personenschaden und schwerwiegenden Unfällen mit Sachschaden zugrundegelegt ${ }^{91}$. Die Unfallzahlen der Straßenbahnunfälle entstammen ebenfalls der Straßenverkehrsunfallstatistik ${ }^{92}$. Die Verunglücktenzahlen der Straßenbahnunfälle sind der Schienenverkehrsunfallstatistik entnommen ${ }^{93}$.

Die Kennzahlen der Fahrleistung und Beförderungsleistung entstammen der Software TREMOD ${ }^{94}$. TREMOD wurde vom Umweltbundesamt entwickelt und dient der Berechnung der Luftschadstoff und Klimagasemissionen aus dem motorisierten Verkehr in Deutschland. Die dafür hinterlegten Fahr- und Beförderungsleistungen der Verkehrsmittel wurden vom Umweltbundesamt für das vorliegende Forschungsvorhaben zur Verfügung gestellt.

Zur Berechnung der Unfallkosten wurden die pauschalen Unfallkostensätze der Bundesanstalt für Straßenwesen (BASt) mit Preisstand 2009 angenommen. ${ }^{95}$

Die zur Berechnung zugrundegelegten Eingangsgrößen sind in Tabelle 4-18 und Tabelle 4-19 zusammengefasst.

\footnotetext{
1 [DESTATIS 2010], [DESTATIS 2011], [DESTATIS 2012], jeweils Tabelle UJ 47

[DESTATIS 2010], [DESTATIS 2011], [DESTATIS 2012], jeweils Tabelle UJ 6 (5)

[DESTATIS 2013a]

[TREMOD 2013]

[BASt 2011], S. 1
} 
Tabelle 4-18: zusammengefasste Eingangsgrößen des Verkehrsmittelvergleichs

\begin{tabular}{|c|c|c|c|c|}
\hline Kennzahl & Einheit & $\begin{array}{l}\text { mit Beteiligung } \\
\text { PKW, innerorts }\end{array}$ & $\begin{array}{c}\text { mit Beteiligung } \\
\text { Bus, innerorts }\end{array}$ & $\begin{array}{l}\text { mit Beteiligung } \\
\text { Straßenbahn }\end{array}$ \\
\hline Unfälle & Anzahl /a & 215.979 & 5.397 & 1.814 \\
\hline \begin{tabular}{l|l} 
mit Getöteten \\
\end{tabular} & Anzahl /a & 690 & 27 & 36 \\
\hline mit Schwerverletzten & Anzahl /a & 23.558 & 706 & 340 \\
\hline mit Leichtverletzten & Anzahl /a & 142.386 & 4.026 & 1.054 \\
\hline mit schwerw. Sachschaden & Anzahl /a & 49.346 & 638 & 385 \\
\hline Verunglückte & Anzahl /a & 205.928 & 7.286 & 1.463 \\
\hline \begin{tabular}{l|l} 
& Getötete \\
\end{tabular} & Anzahl /a & 719 & 29 & 41 \\
\hline Schwerverletzte & Anzahl /a & 25.394 & 784 & 210 \\
\hline Leichtverletzte & Anzahl /a & 179.815 & 6.473 & 1.212 \\
\hline Unfallkosten & Mio. € & 7611 & 224 & 99 \\
\hline Fahrleistung & Mrd. Fzg.-km & 182,34 & 1,08 & 0,30 \\
\hline Beförderungsleistung & Mrd. P.-km & 271,61 & 18,53 & 16,48 \\
\hline
\end{tabular}

Tabelle 4-19: Kostensätze als Grundlage zur Ermittlung der absoluten Unfallkosten Quelle: $\quad$ [BASt 2011], S. 1

\begin{tabular}{|lr|}
\hline Kostensätze für Personenschäden (je verunglückte Person) \\
Getötete & 996.412 \\
Schwerverletzte & 110.571 \\
Leichtverletzte & 4.416 \\
& \\
Kostensätze für Sachschäden (je Unfall) & \\
Unfall mit Getöteten & 40.108 \\
Unfall mit Schwerverletzten & 19.215 \\
Unfall mit Leichtverletzten & 13.036 \\
Schwerwiegender Unfall mit nur Sachschaden & 19.365 \\
\hline
\end{tabular}

Bei der Betrachtung der absoluten Unfallzahlen ist zu erkennen, dass Unfälle mit Beteiligung von Straßenbahnen vergleichsweise seltener sind (vgl. Tabelle 4-18). Auch die Zahl der Schwer- und Leichtverletzen ist absolut betrachtet bei Straßenbahnen im Vergleich gering. Bei Betrachtung der Zahl der Getöteten ist allerdings zu erkennen, dass diese bei Straßenbahnen höher ist, als bei Bussen. Das lässt insgesamt auf eine höhere Unfallschwere bei Unfällen mit Beteiligung von Straßenbahnen schließen.

Diese Annahme wird bei dem Vergleich der Kennzahlen für die Unfallschwere in Abbildung 4-1 und Abbildung 4-2 bestätigt. Die Unfallschwere bei Straßenbahnunfällen ist deutlich höher als bei Unfällen mit PKW und Bussen. Insbesondere bei der Anzahl der Getöteten pro 1.000 Unfälle mit Personen oder schwerwiegendem Sachschaden liegt die Zahl bei Straßenbahnen um das ca. 4-fache höher als bei Bussen und um das ca. 7-fache höher als bei PKW. 


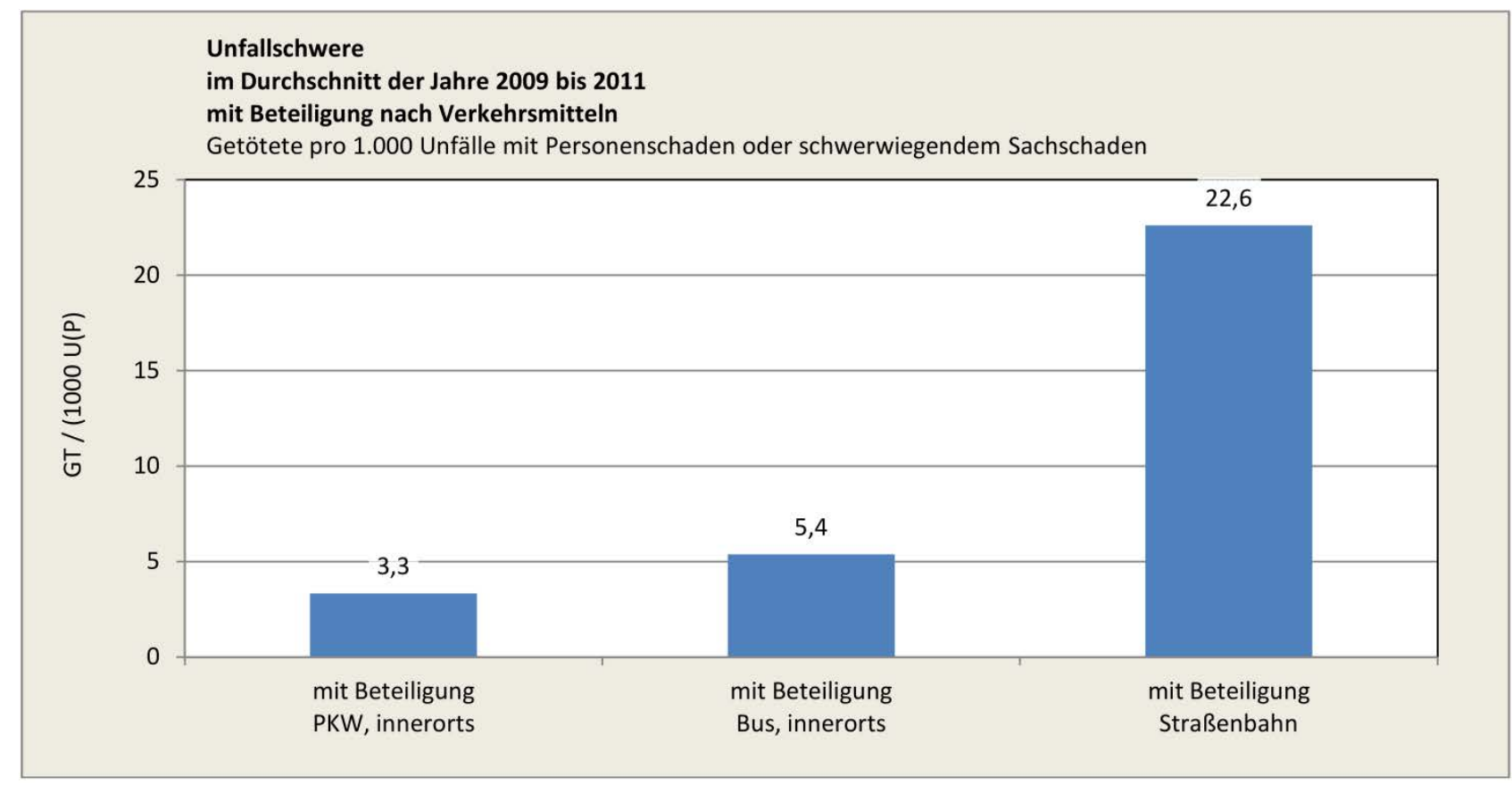

Abbildung 4-1: Verkehrsmittelvergleich nach Unfallschwere [Getötete pro 1.000 Unfälle mit Personenschaden oder schwerwiegendem Sachschaden]

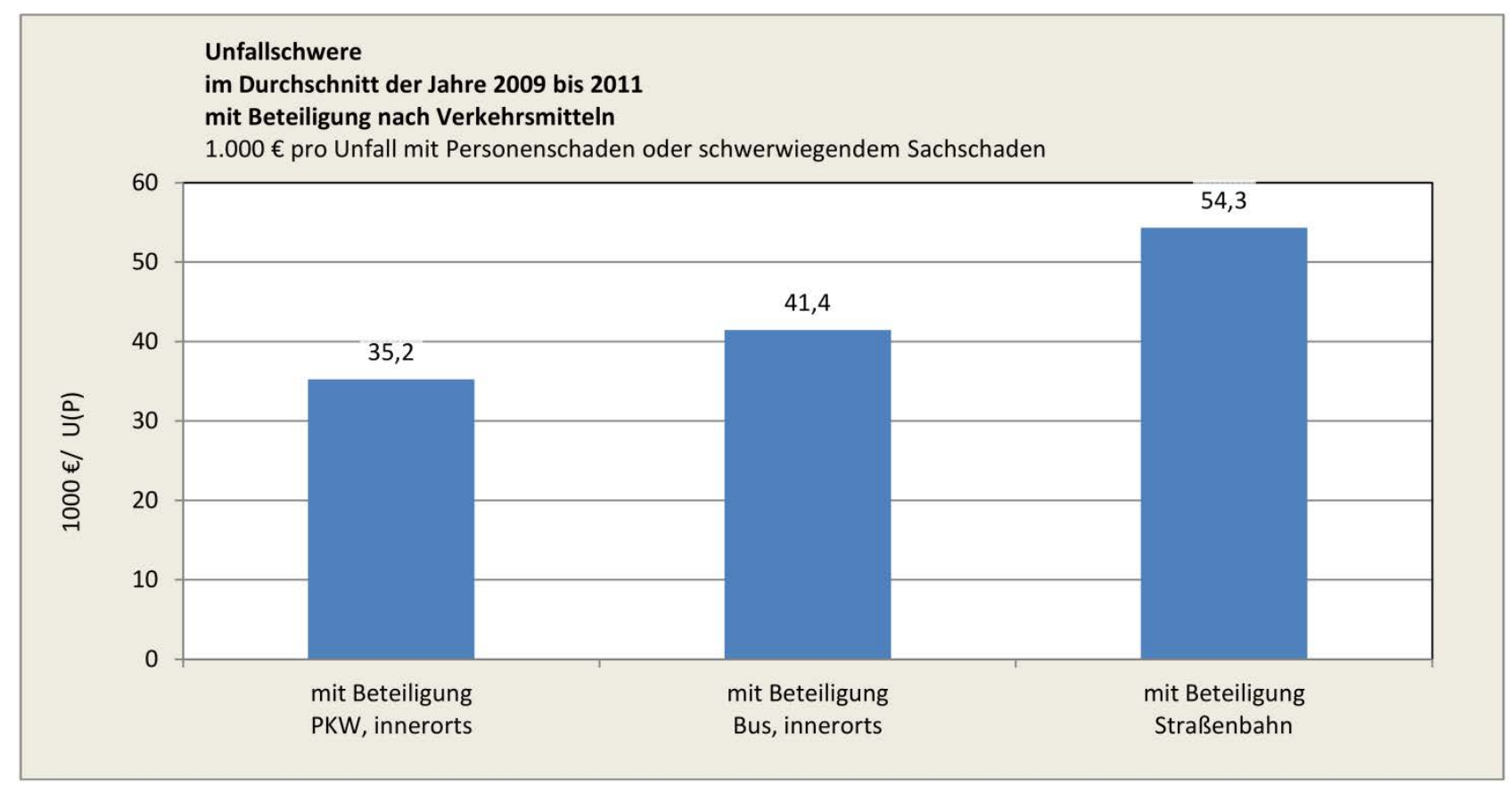

Abbildung 4-2: Verkehrsmittelvergleich nach Unfallschwere [1.000 $€$ pro Unfall mit Personenschaden oder schwerwiegendem Sachschaden]

Des Weiteren wurde wie eingangs beschrieben, eine relative Bewertung der Absolut-Zahlen zum einen durch eine fahrleistungsbezogene, zum anderen durch eine beförderungsleistungsbezogene Gegenüberstellung durchgeführt.

Der relative Bezug auf die Fahrleistung berücksichtigt, dass Straßenbahnen im Vergleich, insbesondere zum PKW, aber auch zum Bus eine wesentlich geringere Fahrleistung in der Summe 
aufweisen. Die damit verbundene geringere Unfallwahrscheinlichkeit wird somit in der Bewertung durch die Berechnung der Unfallrate bzw. Unfallkostenrate erfasst.

Soll im Folgenden noch berücksichtigt werden, dass der Personenbeförderung mit Straßenbahnen eine höhere beförderungswirksamere Bedeutung zukommt, die nur durch eine Vielzahl von zusätzlichen Fahrten durch Busse und noch mehr durch PKW kompensiert werden könnte, dann ist der beförderungsleistungsbezogene Vergleich heranzuziehen.

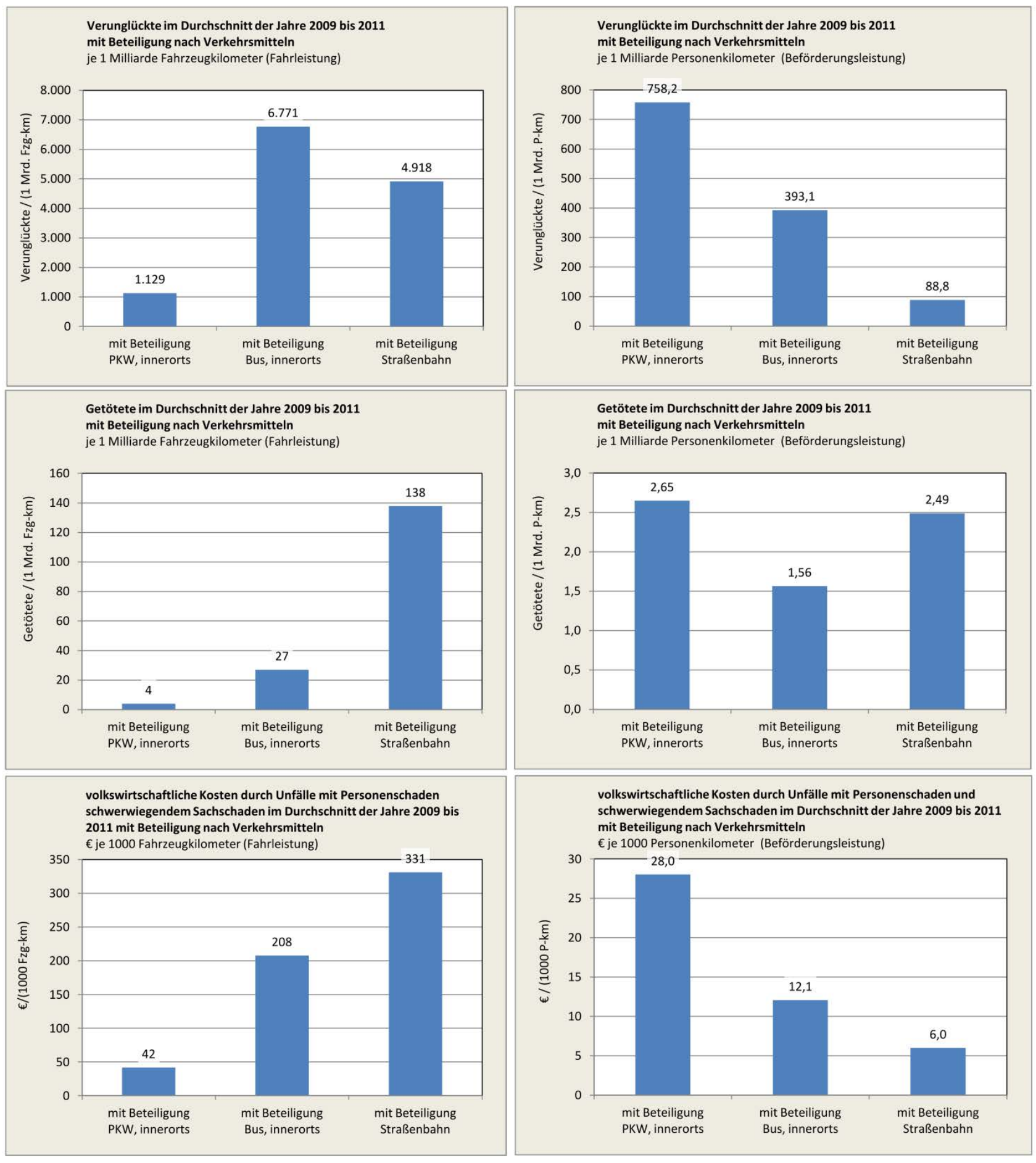

Abbildung 4-3: fahrleistungsbezogener und beförderungsleistungsbezogener Verkehrsmittelvergleich

Die beiden Bezugsgrößen Fahrzeugkilometer und Beförderungskilometer ergeben für die berechneten Kenngrößen ein sehr unterschiedlich zu bewertendes Ergebnis. Grund dafür ist die 
hohe Besetzung mit Fahrgästen in Straßenbahnen, sodass im Vergleich zum PKW oder Bus bei weniger gefahrenen Kilometern eine größere Anzahl an Personen befördert werden können.

Bezogen auf die gefahrenen Kilometer ist die Straßenbahn ein vergleichsweise unsicheres Fahrzeug (vgl. Abbildung 4-3, volkswirtschaftliche Kosten in $€$ je 1.000 Fahrzeugkilometer). Es ist zu erkennen, dass die Unfallkostenrate bei Straßenbahnen im Vergleich zum PKW um das 8-fache höher liegt (331 €/ 1.000 Fzg.-km bei Straßenbahnen; $42 € / 1.000$ Fzg.-km bei PKW).

Bezogen auf die beförderten Personen ist die Straßenbahn ein vergleichsweise sicheres Fahrzeug (vgl. Abbildung 4-3, volkswirtschaftliche Kosten in $€$ je 1.000 Personenkilometer). Es ist zu erkennen, dass das Unfallkostenrisiko bei Straßenbahnen $20 \%$ von dem der PKW ist $(6 € / 1.000$ P.-km bei Straßenbahnen; $28 € / 1.000$ P.-km bei PKW).

Anders ausgedrückt können folgende Thesen aufgestellt werden:

- Straßenbahnen sind ein sicheres Transportmittel, da sich - bezogen auf die vielen beförderten Personen - vergleichsweise wenige Unfälle mit Personenschäden ereignen.

- Zugleich sind Straßenbahnen aber auch ein vergleichsweise weniger sicheres Verkehrsmittel, da sich - bezogen die gefahrenen Kilometer einer Straßenbahn - vergleichsweise viele und vergleichsweise schwere Unfälle mit Personenschäden ereignen. 


\section{$5 \quad$ Sicherheitsanalyse von Straßenbahnnetzen in ausgewählten Städten}

Um die aus Kapitel 4 erkannten Verkehrs(un)sicherheiten zu präzisieren, erfolgt in diesem Kapitel die Erläuterung zu einer Analyse von Straßenbahnnetzen ausgewählter Städte. Ziel der Untersuchung ist es, differenzierte Unfallkenngrößen und strukturelle Unterschiede für unterschiedliche Querschnittstypen und RASt-Entwurfssituationen zu erhalten.

Zu diesem Zweck wurde für fünf Städte das Straßenbahnliniennetz bezüglich der Merkmalsausprägungen für die Querschnittstypen und RASt-Entwurfssituationen in einem Geoinformationssystem (GIS) nachskizziert, um die längenbezogenen Merkmalsverteilungen zu erhalten.

Folgende Städte wurden in der Netzanalyse betrachtet:

Tabelle 5-20: $\quad$ Städte der Netzanalyse mit Übersichtskarten der georeferenzierten Unfälle Quelle: $\quad$ eigene Darstellung; Kartengrundlage: ESRI, ArcMap 10.0 (openstreetmap)

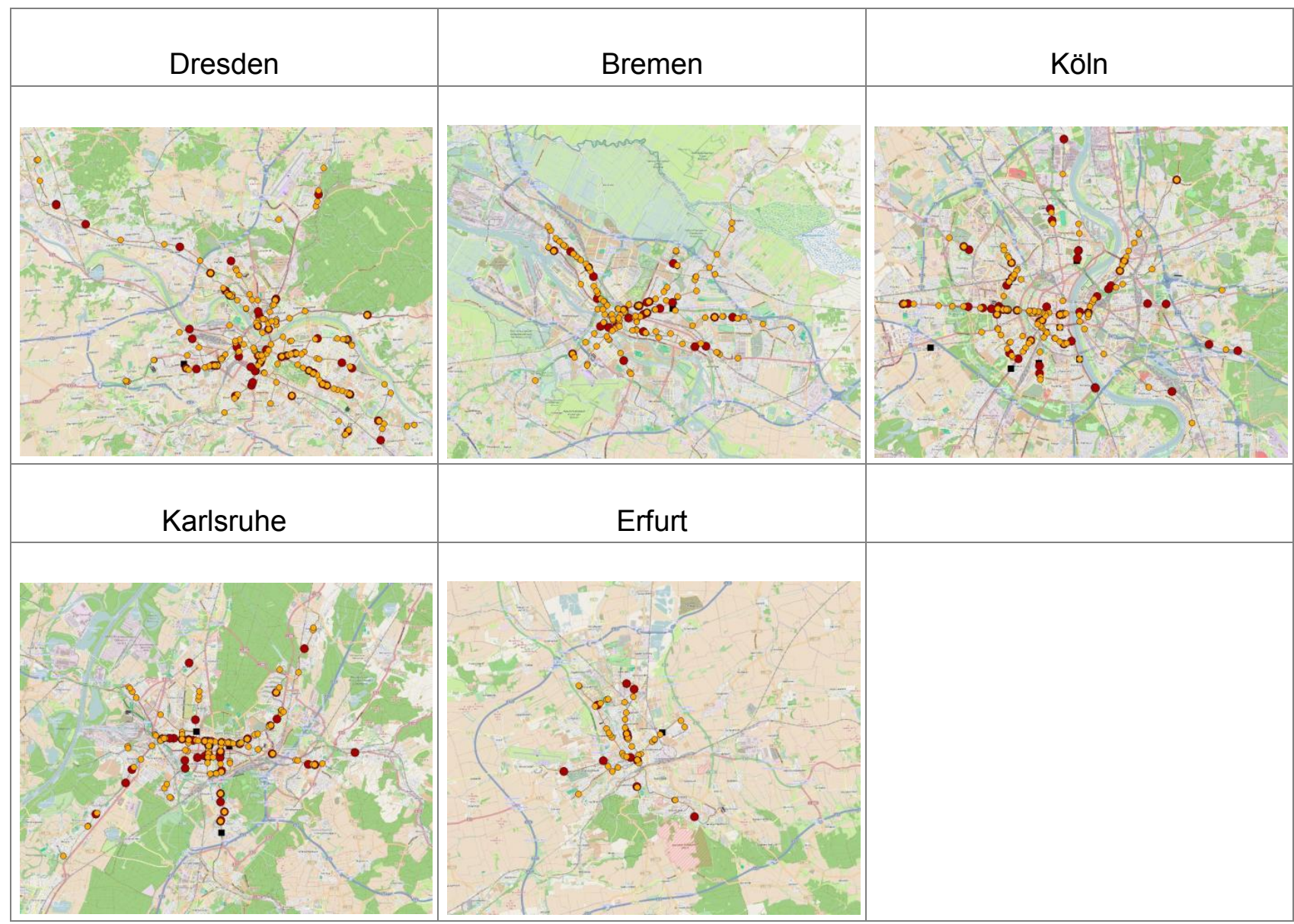

Die Städte sollten sich hinsichtlich ihrer regionalen Lage als auch hinsichtlich ihrer Größe unterscheiden. 
Basis der Unfalldaten bildeten die georeferenzierten, polizeilich erfassten Unfalldaten, die wie in Kapitel 6.1 .2 beschrieben, erhoben und aufbereitet wurden.

Diese Unfalldaten wurden in das Geoinformationssystem (GIS) ArcGIS ${ }^{96}$ importiert. Das jeweilige Liniennetz einer Stadt wurde im GIS vollständig nachskizziert, wobei entsprechend der Merkmalsverteilung nach Querschnittstyp und RASt-Entwurfssituation (vgl.Kapitel 6.1.3) Netzsegmente erstellt wurden.

Insgesamt wurden auf diese Weise für die fünf Städte je zwei georeferenzierte Straßennetze auf das jeweilige Liniennetz übertragen.

Die Unfälle wurden den Netzsegmenten zugeteilt. Mit den Prozeduren des GIS war somit bekannt, welche Unfälle in welcher Schwere sich auf den Netzabschnitten mit welchen jeweiligen Merkmalseigenschaften befanden.

Darauf aufbauend konnten die Netzabschnitte mit der selben Merkmalsausprägung zusammengefasst werden und die dazugehörigen Unfallkenngrößen sowie weitere strukrurelle Verteilungen berechnet werden.

Aus den Auswertungen von Kapitel 6.2.1 wird ersichtlich, dass sich die überwiegende Anzahl der Unfälle mit Beteiligung von Straßenbahnen an Knotenpunkten ereignet. Aus diesem Grund wurden in die Netzbewertung zwei verschiedene Unfallkollektive berücksichtigt: in einer ersten Auswertung wurden alle vorliegenden Unfälle mit Personenschaden der Städte in die Bewertung eingbezogen, damit für die verschiedenen Differenzierungsmerkmale eine vertrauenswürdige Stichprobenmenge zugrundeliegt. In einer zweiten Auswertung wurden ausschließlich Unfälle berücksichtigt, die größer/ gleich 25 m von einem Knotenpunkt entfernt liegen, um die Unfallkenngrößen und strukturellen Verteilungen ausschließlich der Streckenabschnitte bewerten zu können.

Die wesentlichen Ergebnisse sind in den folgenden Abbildungen zusammengefasst. Die Darstellungen aller erstellten Diagramme finden sich im Anhang A.7.

\section{Straßenbahnunfälle mit Personenschäden einschließlich Knotenpunktunfälle}

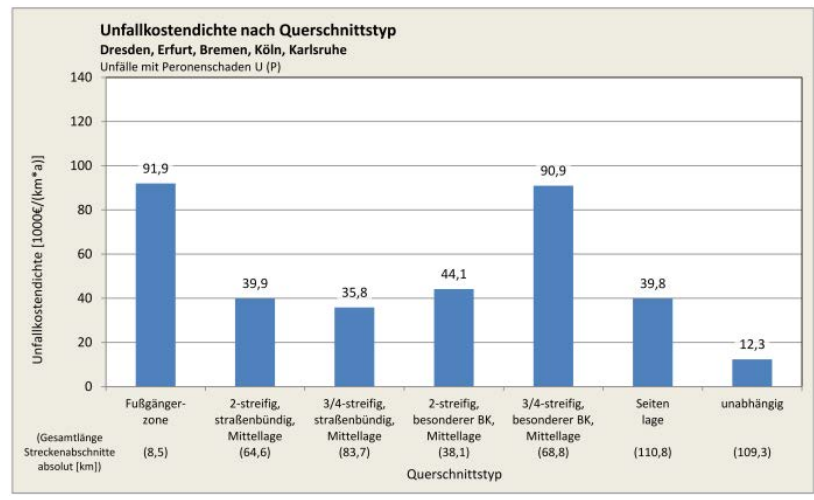

Abbildung 5-4: Unfallkostendichte nach Querschnittstyp, alle $\mathbf{U}_{\text {Strab }}(\mathbf{P})$

\section{Straßenbahnunfälle mit Personenschäden ohne Knotenpunktunfälle}

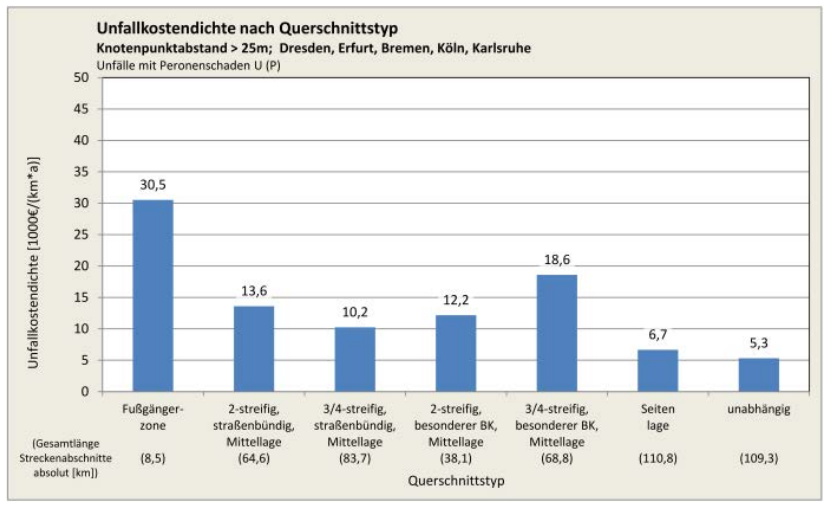

Abbildung 5-5: Unfallkostendichte nach Querschnittstyp, nur $U_{\text {Strab }}(P)$ außerhalb v. Knotenpunkten

96 ArcGIS, ArcMap Version 10.0, ESRI Inc. 


\section{Straßenbahnunfälle mit Personenschäden einschließlich Knotenpunktunfälle}

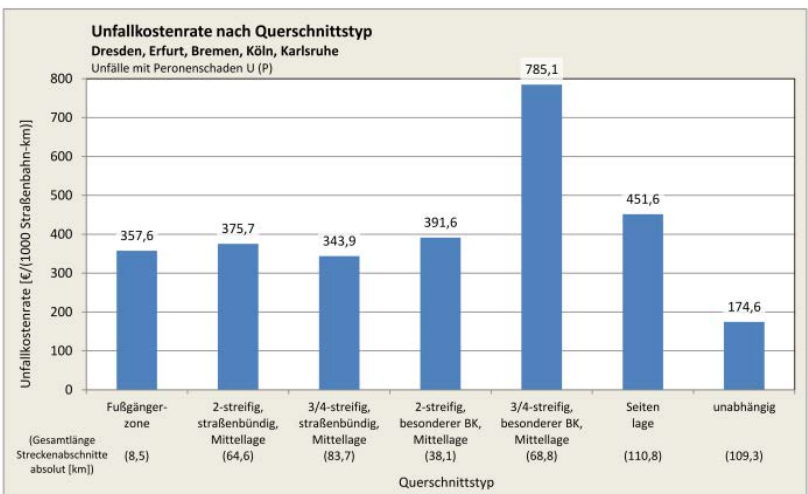

Abbildung 5-6: Unfallkostenrate nach Querschnittstyp, alle $\mathbf{U}_{\text {Strab }}(P)$

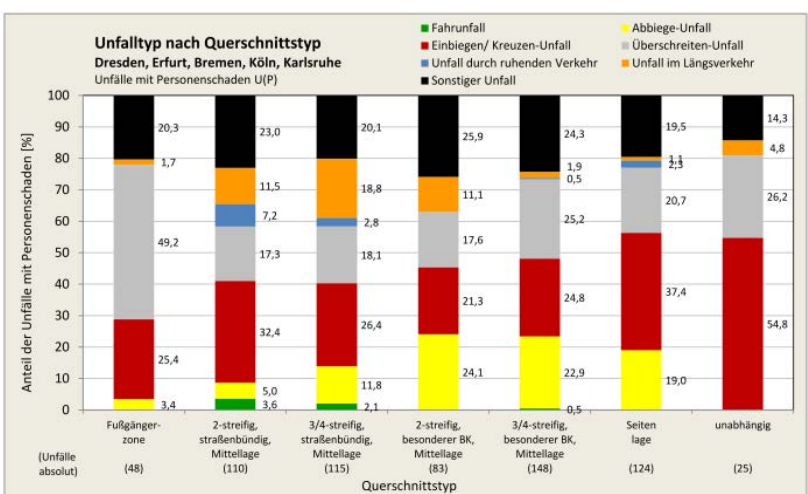

Abbildung 5-8: Unfalltyp nach Querschnittstyp, alle $U_{\text {Strab }}(P)$

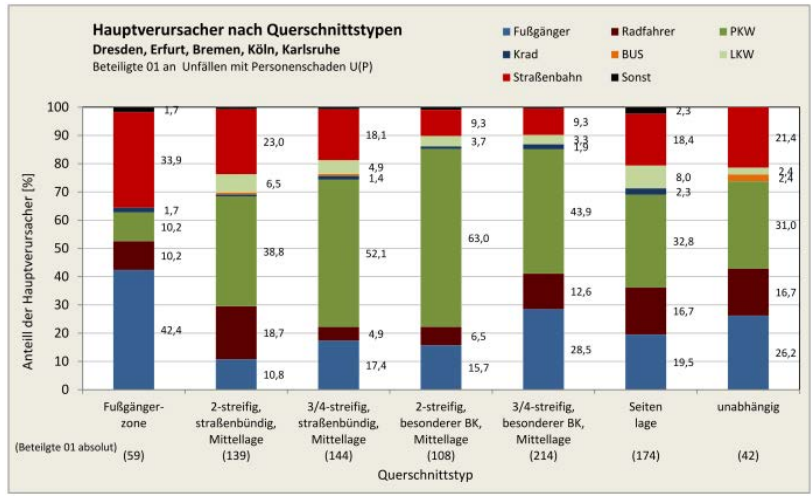

Abbildung 5-10: Hauptverursacher nach Querschnittstyp, alle $\mathbf{U}_{\text {Strab }}(\mathbf{P})$

\section{Straßenbahnunfälle mit Personenschäden ohne Knotenpunktunfälle}

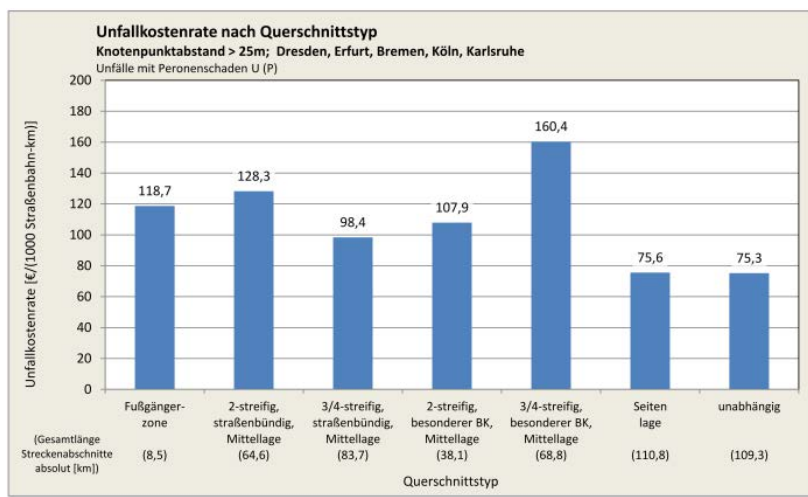

Abbildung 5-7: Unfallkostenrate nach Querschnittstyp, nur $U_{\text {Strab }}(P)$ außerhalb v. Knotenpunkten

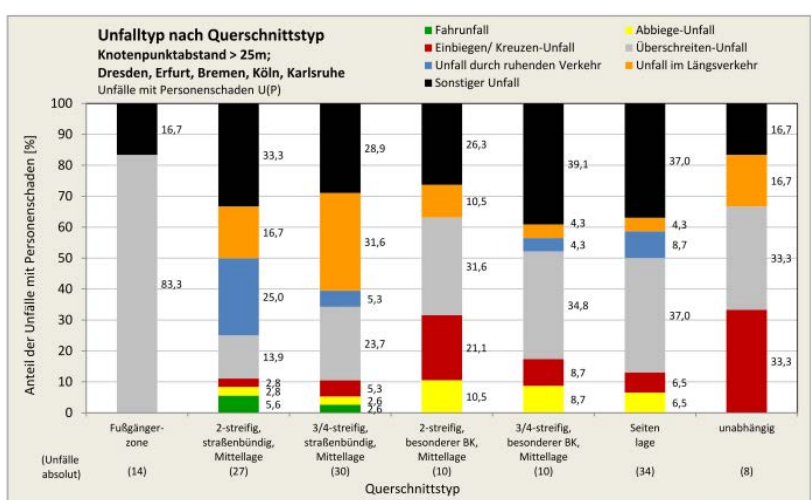

Abbildung 5-9: Unfalltyp nach Querschnittstyp, nur $U_{\text {Strab }}(P)$ außerhalb v. Knotenpunkten

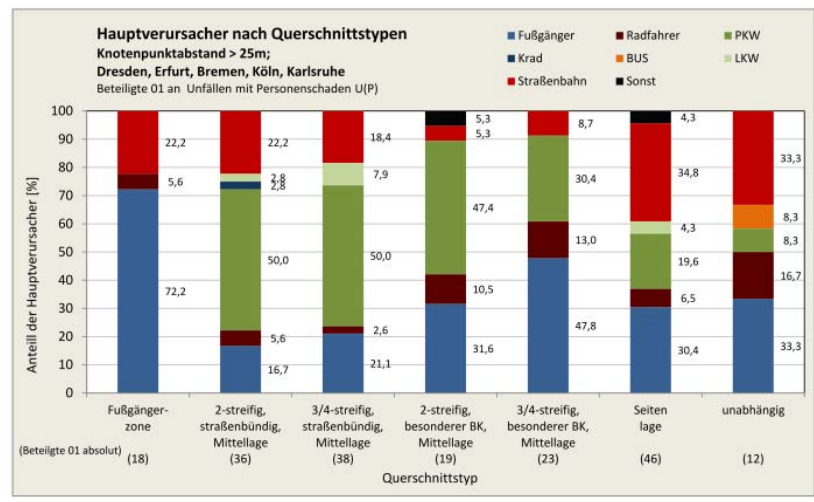

Abbildung 5-11: Hauptverursacher nach Querschnittstyp, nur $U_{\text {Strab }}(P)$ außerhalb v. Knotenpunkten

Die Unfallkostenrate in Abbildung 5-6 bzw. Abbildung 5-7 kann als aussagekräftige Unfallkenngröße für die Bewertung der Verkehrs(un)sicherheit zugrundegelegt werden (vgl. Kapitel 4).

Abbildung 5-6 zeigt deutlich, dass der 3- bzw. 4-streifige Querschnitt mit besonderem Bahnkörper in Mittellage hinsichtlich der Unfallfolgen besonders auffällig ist, selbst dann, wenn nur die Unfälle außerhalb von Knotenpunkten betrachtet werden (Abbildung 5-7). Die makroskopische Unfallanalyse weist zudem hier eine besonders hohe Unfallschwere auf.

Der unabhängige Bahnkörper zeigt sich insgesamt als sicherster Querschnittsstyp, auch wenn die Unfälle, die hier geschehen besonders schwer sind (s. Abbildung 6-34). Allerdings ist diese Querschnittsform eher der Lage nach dem Stadtrandgebiet mit weniger Querverkehr zuzuordnen. 
Bei Erschließung und Neuanlage neuer Wohngebiete ist aber auch denkbar, dass

Straßenbahnnetz als unabhängige Bahnköper abseits des Straßennetzes zu konzipieren und die Haltestellen an den Wegekreuzungen mit dem Straßennetz anzulegen.

Der Querschnitt mit Führung der Straßenbahn in Seitenlage zeigt unter Berücksichtigung der Knotenpunktunfälle keinen wesentlichen Sicherheitsgewinn. Außerhalb der Knotenpunkte ist dieser Querschnitt allerdings ähnlich sicher wie der unabhängige Bahnkörper.

Unter Berücksichtigung der Untersuchungen von BAIER/ MAIER, bei der auch Unfälle des KfzVerkehrs betrachtet wurden, können der unabhängige Bahnkörper und der Bahnkörper in Seitenlage als die sichersten Führungsformen von Straßenbahnen bewertet werden.

\section{Straßenbahnunfälle mit Personenschäden einschließlich Knotenpunktunfälle}

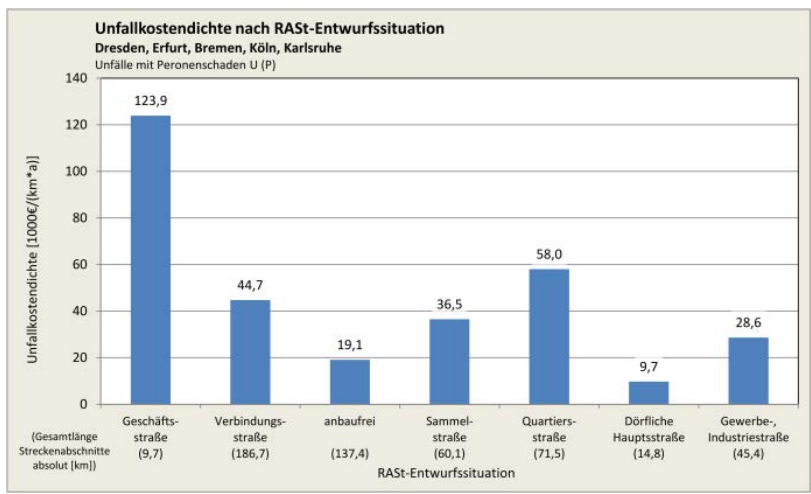

Abbildung 5-12: Unfallkostendichte nach Entwurfssituation, alle $U_{\text {Strab }}(P)$

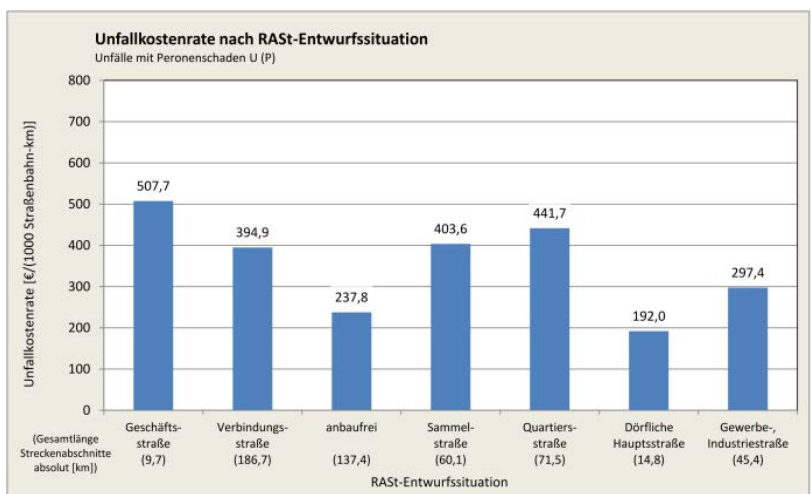

Abbildung 5-14: Unfallkostenrate nach Entwurfssituation, alle $U_{\text {Strab }}(P)$

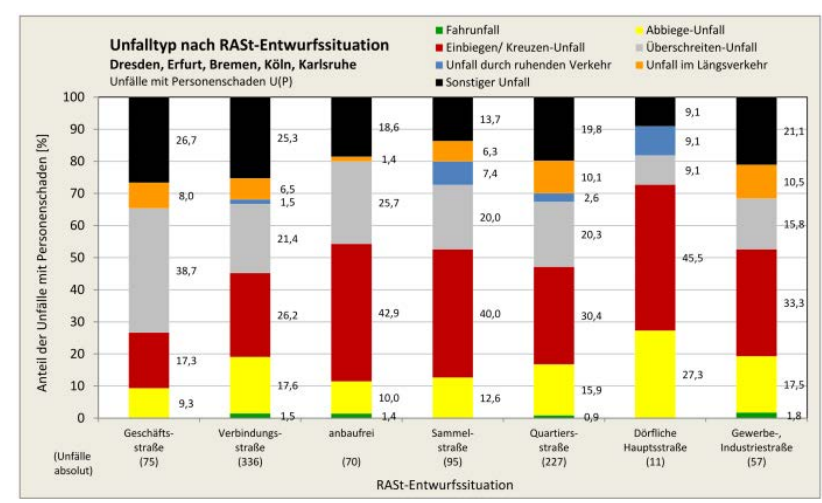

Abbildung 5-16: Unfalltyp nach Entwurfssituation, alle $U_{\text {strab }}(P)$

\section{Straßenbahnunfälle mit Personenschäden ohne Knotenpunktunfälle}

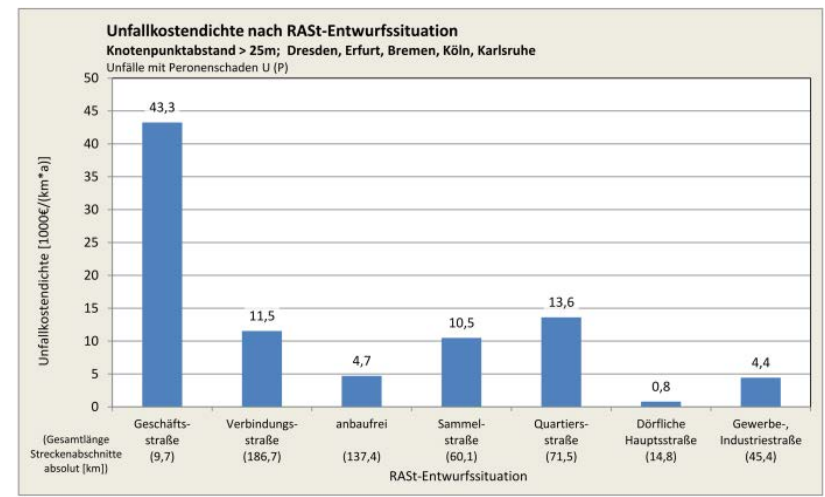

Abbildung 5-13: Unfallkostendichte nach Entwurfssituation, nur $U_{\text {strab }}(P)$ außerhalb v. Knotenpunkten

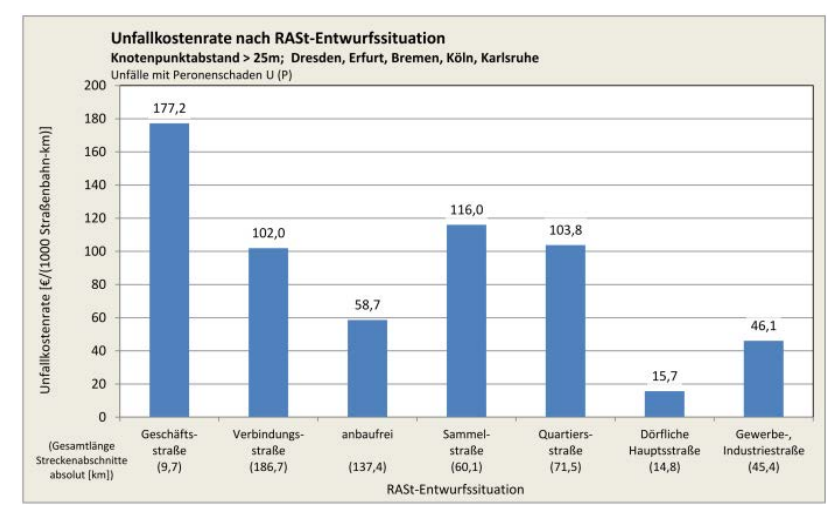

Abbildung 5-15: Unfallkostenrate nach Entwurfssituation, nur $U_{\text {strab }}(P)$ außerhalb v. Knotenpunkten

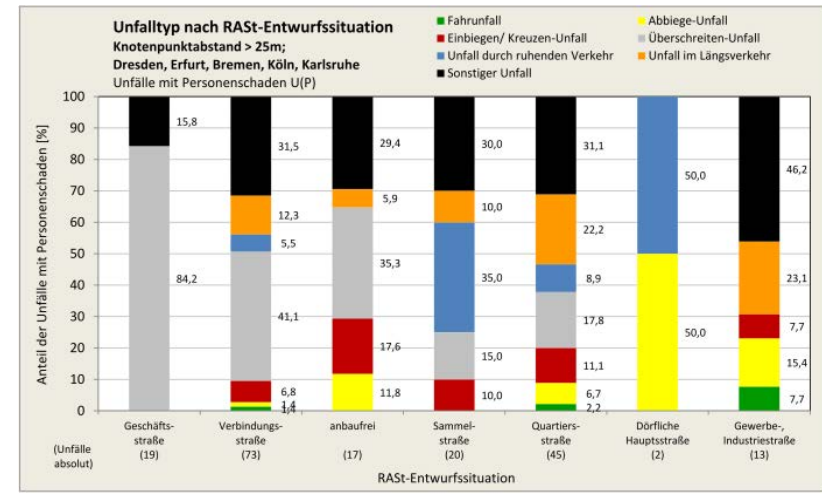

Abbildung 5-17: Unfalltyp nach Entwurfssituation, nur $U_{\text {Strab }}(P)$ außerhalb v. Knotenpunkten 


\section{Straßenbahnunfälle mit Personenschäden einschließlich Knotenpunktunfälle}

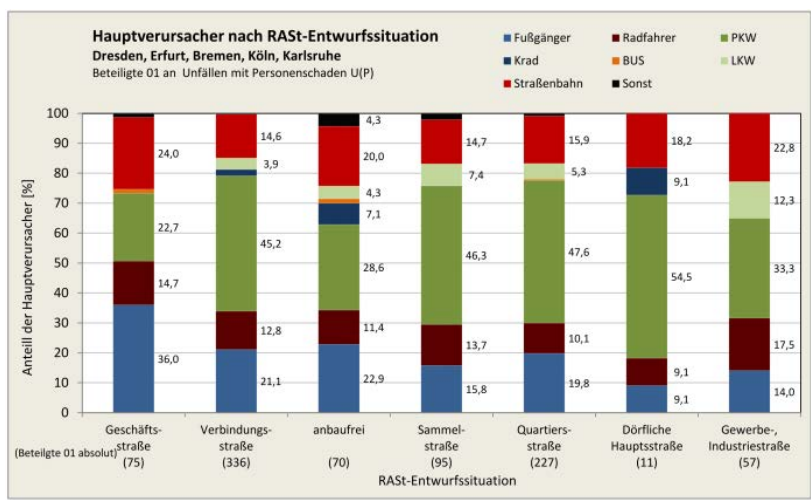

Abbildung 5-18: Hauptverursacher nach Entwurfssituation, alle $U_{\text {Strab }}(P)$

\section{Straßenbahnunfälle mit Personenschäden ohne Knotenpunktunfälle}

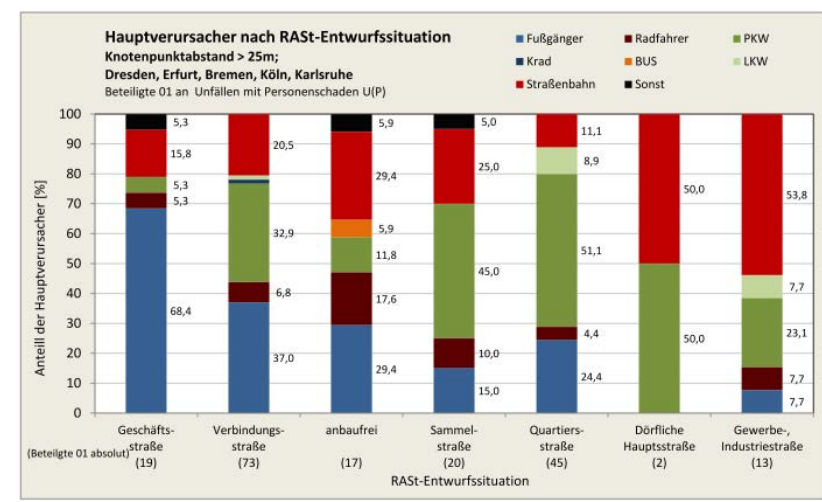

Abbildung 5-19: Hauptverursacher nach Entwurfssituation, nur $U_{\text {Strab }}(P)$ außerhalb v. Knotenpunkten

Bei der Bewertung des Netzes nach der RASt-Entwurfssituation zeigt sich, dass Geschäftsstraßen vergleichsweise hohe Unfallkostendichten und Unfallkostenraten aufweisen. Insbesondere der hohe Anteil von Überschreiten-Unfällen ist dabei auffällig.

Dem gegenüber stehen die geringen Unfallkostendichten bzw. Unfallkostenraten von Dörflichen Hauptstraßen und Gewerbe- bzw. Industriestraßen, bei denen nur in geringem Maße Überschreiten-Unfälle zu verzeichnen sind. Das lässt die Vermutung zu, dass für das Gesamtunfallgeschehen mit Straßenbahnen das sichere Queren von Fußgängern einen wesentlichen Einfluss hat. Diese Annahme wird in Kapitel 6.2.1 weiter bestärkt.

\section{Auf der Netzanalyse aufbauende Empfehlungen}

- Bei Neukonzeption und Neubau von Straßenbahnnetzen und Netzteilen sollte der unabhängige Bahnkörper oder der Bahnkörper in Seitenlage verstärkt umgesetzt werden.

- Bei Neubau und grundhaften Umbauplanungen von Straßen mit Straßenbahnbetrieb sollten Bahnkörper in Seitenlage verstärkt umgesetzt werden.

\section{Bahnkörper in Seitenlage}

- Bei Bahnkörpern in Seitenlage ist bei Entwurf und Betrieb von Knotenpunkten den abbiegenden, einbiegenden und kreuzenden Kraftfahrzeugen und Radfahrern erhöhte Sorgfalt einzuräumen,

\section{z.B. durch festgestellte Beispiellösungen im Rahmen der Ortsbesichtigung:}
Signalisierung von Knotenpunkten jeglichen Typs ab 100 Kfz/ Tag (auch Grundstückszufahrten),
- Wiederholung des Richtungssignals über der Fahrbahn abbiegender Kfz,
- Zeit- oder Wegevorsprung für Straßenbahnen mit konfligierenden Strömen bei widerrechtlichem Befahren,
- Zusätzliche Signalgeber für Radfahrer bei gleichzeitiger Signalisierung mit dem Kfz-Verkehr.

- Bei Bahnkörpern in Seitenlage ist bei Entwurf und Betrieb der freien Strecke querenden Fußgängern und Radfahrern erhöhte Sorgfalt einzuräumen,

z.B. durch festgestellte Beispiellösungen im Rahmen der Ortsbesichtigung: 
- Zusätzliche Sicherungseinrichtungen (akustische Signale, Signalgeber in geringer Höhe) bei unübersichtlichen Querungsstellen, gradliniger Führung, hohem Querungsbedarf,

- Schaffung von Sichtfeldern zur Erhöhung der Wahrnehmbarkeit bevorrechtigter Straßenbahnfahrzeuge.

\section{Bahnkörper in Mittellage}

- Bei besonderen Bahnkörpern in Mittellage ist auf der freien Strecke und an Knotenpunkten, insbesondere den querenden Fußgängern und Radfahrern und abbiegenden Kraftfahrzeugen an Knotenpunkten, erhöhte Sorgfalt einzuräumen,

z.B. durch festgestellte Beispiellösungen im Rahmen der Ortsbesichtigung:

- Lage und Anzahl der Querungshilfen bei entsprechendem Bedarf,

- Linienhafte Querungsmöglichkeiten bei Geschäftsstraßen,

- Z-Übergänge und Signalisierung mit geringen Verlustzeiten für die Fußgänger,

- Umwegefreie Haltestellenzugänge,

- Zeit- oder Wegevorsprung für Straßenbahnen mit konfligierenden Strömen bei widerrechtlichem Befahren.

\section{$\underline{\text { Straßenbündige Bahnkörper }}$}

- Bei straßenbündigen Bahnkörpern ist auf der freien Strecke und an Knotenpunkten, insbesondere dem Längsverkehr und ruhendem Verkehr, erhöhte Sorgfalt einzuräumen, z.B. durch festgestellte Beispiellösungen im Rahmen der Ortsbesichtigung:

- Erkennbarkeit Begreifbarkeit von Haltestellen oder Knotenpunkten,

- Frühzeitige bauliche Trennung der Richtungsfahrstreifen an Haltestellen,

- Keine Senkrechtparkstände an Fahrstreifen mit Straßenbahnen.

\section{$\underline{\text { Unabhängige Bahnkörper }}$}

- Bei unabhängigen Bahnkörpern ist bei Entwurf und Betrieb von Knotenpunkten den kreuzenden Kraftfahrzeugen und Radfahrern und querenden Fußgängern erhöhte Sorgfalt einzuräumen,

z.B. durch festgestellte Beispiellösungen im Rahmen der Ortsbesichtigung:

- Erkennbarkeit von Bahnübergängen,

- Schaffung von Sichtfeldern zur Erhöhung der Wahrnehmbarkeit bevorrechtigter Straßenbahnfahrzeuge.

- Zusätzliche Signalgeber für Radfahrer bei gleichzeitiger Signalisierung mit dem Kfz-Verkehr.

- Bei unabhängigen Bahnkörpern ist auf der freien Strecke, insbesondere den querenden Fußgängern und Radfahrern, erhöhte Sorgfalt einzuräumen,

z.B. durch festgestellte Beispiellösungen im Rahmen der Ortsbesichtigung:

- Signalisierung und Umlaufgitter insbesondere im Zuge von Radverkehrsachsen,

- Schaffung von Sichtfeldern zur Erhöhung der Wahrnehmbarkeit bevorrechtigter Straßenbahnfahrzeuge. 


\section{$6 \quad$ Makroskopische Analyse}

\subsection{Datengrundlage}

\subsubsection{Art und Umfang der zugrunde gelegten Daten (Datenkollektive)}

In Deutschland konnten insgesamt 60 Städte und Gemeinden in 14 Bundesländern mit Straßenbahnbetrieb nach BOStrab identifiziert werden. Bis auf Schleswig-Holstein und Hamburg haben die übrigen Länder der Bundesrepublik Deutschland wenigstens eine Stadt, in der Straßenbahnbetrieb vorzufinden ist.

Nach Angaben des Verbandes Deutscher Verkehrsunternehmen (VDV) beläuft sich das Gesamtstreckennetz aller deutschen Straßenbahnunternehmen auf zusammen ca. $3.400 \mathrm{~km}$ mit einer zu registrierenden Fahrleistung von 250 Mio. Zugkilometer pro Jahr ${ }^{97}$. Jährlich werden ca. 1.400 Unfälle mit Personenschaden mit Beteiligung von Straßenbahnen statistisch registriert.

Nach dem "Gesetz über die Statistik für Bundeszwecke" ist das Statistische Bundesamt für die Erhebung, Aufbereitung, Sammlung und Veröffentlichung von statistischen Daten der Bundestatistik in Deutschland zuständig.

Statistische Daten im Zusammenhang mit Straßenbahnunfällen werden durch zwei, voneinander unabhängige Erhebungen des Statistischen Bundesamt erfasst. Zum einen werden in der Statistik der Straßenverkehrsunfälle die durch die Polizei aufgenommenen Verkehrsunfalldaten registriert. Dem gegenüber steht die Schienenverkehrsunfallstatistik, bei der die Netzbetreiber respektive die Verkehrsunternehmen aufgefordert werden, die Unfalldaten zu melden. Aufgrund der unterschiedlichen Erhebungsmethodik sind Unterschiede bei den erfassten Unfällen möglich. Auch im Umfang der gemeldeten Daten gibt es wesentliche Unterschiede: Bei der Straßenverkehrsunfallstatistik werden eine Vielzahl geschlossen zu beantwortende Unfallmerkmale bundeseinheitlich mittels der Verkehrsunfallanzeige durch die Polizei vor Ort unfallfein erfasst. Die gemeldeten Daten der Schienenverkehrsunfallstatistik sind dem gegenüber bereits aggregierte Unfallzahlen, deren Umfang an differenzierten Parameter wesentlich geringer gegenüber den der Straßenverkehrsunfallstatistik ist (s. Anhang A.6: Fragebogen Schienenverkehrsstatistik).

Ein Vergleich beider Statistiken im Detail kann der Tabelle 6-21 entnommen werden.

97 vgl. VDV Statistik, [VDV 2011], S. 60-63; Anlage A.3 
Tabelle 6-21: Vergleich der unfallstatistischen Erhebungen des Bundesamtes für Statistik für Straßenbahnen Quellen: vgl. [DESTATIS 2013b], [DESTATIS 2013c]

\begin{tabular}{|c|c|c|}
\hline & Straßenverkehrsunfallstatistik ${ }^{98}$ & Schienenverkehrsunfallstatistik $^{99}$ \\
\hline Berichtszeitraum & Monat und Jahr & Jahr \\
\hline Regionale Gliederung & $\begin{array}{l}\text { Bundesgebiet, Bundesländer, Kreise, } \\
\text { Gemeinden }\end{array}$ & Bundesgebiet \\
\hline \begin{tabular}{|l} 
Erhebungsgesamtheit \\
und \\
Zuordnungsprinzip der \\
Erhebungseinheiten
\end{tabular} & $\begin{array}{l}\text { Straßenverkehrsunfälle, die infolge des } \\
\text { Fahrverkehrs auf öffentlichen Wegen und } \\
\text { Plätzen entstanden sind und die von der } \\
\text { Polizei aufgenommen wurden. }\end{array}$ & $\begin{array}{l}\text { Alle Verkehrsunfälle auf öffentlichen } \\
\text { Schienenwegen/Strecken, bei denen } \\
\text { mindestens ein bewegtes - beim } \\
\text { Zusammenprall auch haltendes - } \\
\text { schienengebundenes Fahrzeug im } \\
\text { Fahrbetrieb auf den gewidmeten Strecken } \\
\text { und Anlagen beteiligt ist. }\end{array}$ \\
\hline $\begin{array}{l}\text { bundesrechtliche } \\
\text { Grundlage }\end{array}$ & $\begin{array}{l}\text { Gesetz über die Statistik der } \\
\text { Straßenverkehrsunfälle } \\
\text { (Straßenverkehrsunfallstatistikgesetz - } \\
\text { StVUnfStatG) vom 15. Juni 1990, letzte } \\
\text { Änderung 31. Oktober } 2006\end{array}$ & $\begin{array}{l}\text { Verkehrsstatistikgesetz (VerkStatG) vom } \\
20 . \text { Februar } 2004 \text { in der jeweils geltenden } \\
\text { Fassung in Verbindung mit dem } \\
\text { Bundesstatistikgesetz (BStatG) vom } 22 . \\
\text { Januar } 1987 \text { in der jeweils geltenden } \\
\text { Fassung }\end{array}$ \\
\hline Erhebungsinhalte & $\begin{array}{l}\text { Ausführliche Angaben zu Unfällen, } \\
\text { Beteiligten, Fahrzeugen, Verunglückten und } \\
\text { Unfallursachen sowie Zahl der Benutzer } \\
\text { unfallbeteiligter Fahrzeuge. }\end{array}$ & $\begin{array}{l}\text { Unfälle mit Personen- oder Sachschaden } \\
\text { nach der Unfallart und die dabei } \\
\text { verletzten oder getöteten Personen nach } \\
\text { der Unfallfolge, der } \\
\text { Verkehrsbeteiligungsart bzw. } \\
\text { Personenkategorie sowie Unfälle mit } \\
\text { Personen- und Sachschaden beim } \\
\text { Transport gefährlicher Güter mit } \\
\text { Gefahrgutaustritt nach der Unfallart. }\end{array}$ \\
\hline $\begin{array}{l}\text { Art der } \\
\text { Datengewinnung }\end{array}$ & $\begin{array}{l}\text { Die Erhebung wird als Vollerhebung } \\
\text { durchgeführt; auskunftspflichtig sind die } \\
\text { Polizeidienststellen, deren Beamte einen } \\
\text { Unfall nach der o.a. Definition aufgenommen } \\
\text { haben. }\end{array}$ & $\begin{array}{l}\text { Die Erhebung wird als Vollerhebung } \\
\text { durchgeführt; auskunftspflichtig sind die } \\
\text { Inhaber(innen) oder die für die Leitung } \\
\text { beziehungsweise Geschäftsführung } \\
\text { verantwortlichen Personen der } \\
\text { Unternehmen, die Schieneninfrastruktur } \\
\text { des öffentlichen Verkehrs im Inland } \\
\text { betreiben. Für das Erhebungsmerkmal } \\
\text { Zahl der Verunglückten mit Todesfolge } \\
\text { (Getötete) sind die Inhaber(innen) oder } \\
\text { die für die Leitung beziehungsweise } \\
\text { Geschäftsführung verantwortlichen } \\
\text { Personen der Unternehmen } \\
\text { auskunftspflichtig, die als Betriebsführer } \\
\text { oder beauftragte Beförderer öffentliche } \\
\text { Personenbeförderung mit Eisenbahnen } \\
\text { oder Straßenbahnen beziehungsweise als } \\
\text { Frachtführer oder als ausführendes } \\
\text { Schienenverkehrsunternehmen auf dem } \\
\text { inländischen Schienennetz des } \\
\text { öffentlichen Verkehrs durchführen. }\end{array}$ \\
\hline
\end{tabular}




\begin{tabular}{|c|c|c|}
\hline & Straßenverkehrsunfallstatistik ${ }^{98}$ & Schienenverkehrsunfallstatistik ${ }^{99}$ \\
\hline $\begin{array}{l}\text { Erhebungsinstrumente } \\
\text { und Berichtsweg }\end{array}$ & $\begin{array}{l}\text { Die Polizeidienststellen melden ihre Angaben } \\
\text { elektronisch oder auf Papier (Durchschlag } \\
\text { der Unfallanzeige) an das jeweilige } \\
\text { zuständige Statistische Landesamt. Diese } \\
\text { übermitteln die Daten an das Statistische } \\
\text { Bundesamt (dezentrale Erhebung). Wegen } \\
\text { des hohen öffentlichen Interesses an den } \\
\text { Unfallzahlen werden vorab monatlich so } \\
\text { genannte Schnellmeldungen zu den } \\
\text { Eckzahlen der Unfälle und Verunglückten } \\
\text { übermittelt. Aus innen entstehen in der Regel } \\
\text { auch die Pressemitteilungen der } \\
\text { Statistischen Ämter. }\end{array}$ & $\begin{array}{l}\text { Die Unternehmen melden ihre Angaben } \\
\text { auf Papier bzw. elektronisch an das } \\
\text { Statistische Bundesamt (zentrale } \\
\text { Erhebung). }\end{array}$ \\
\hline Publikationswege & $\begin{array}{l}\text { Ergebnisse zu dieser Statistik enthält die } \\
\text { Fachserie 8, Reihe } 7 \text { „Verkehrsunfälle“ }\end{array}$ & $\begin{array}{l}\text { Ergebnisse zu dieser Statistik enthält die } \\
\text { Fachserie } 8 \text {, Reihe } 7 \text { „Verkehrsunfälle“ } \\
\text { sowie die Fachserie } 8 \text {, Reihe } 2 \\
\text { „Eisenbahnverkehr" }\end{array}$ \\
\hline
\end{tabular}

Sofern die polizeilich erfassten Unfälle korrekt aufgenommen wurden und die unfallfeinen Daten vollständig zur Verfügung gestellt werden, können diese Unfälle eindeutig lokalisiert werden. Damit sind weitere straßenräumliche Merkmale nachträglich zu erfassen, die nicht unmittelbar Bestandteil der Verkehrsunfallanzeige sind. Aufgrund dieser Tatsache und der darüber hinaus umfangreichen Unfallmerkmale, wurden ausschließlich polizeilich bundeseinheitlich aufgenommene Unfälle in diesem Forschungsvorhaben berücksichtigt.

Als Netzdaten werden im Folgenden all die Daten verstanden, die nicht der Verkehrsunfallanzeige entnommen werden können.

Für das vorliegende Forschungsvorhaben umfassen die Netzdaten folgende Teildaten:

- Verkehrsmengendaten und

- straßenräumliche Merkmale

Weitere Erläuterungen zur Erfassung, Aufbereitung und Auswertung der Netzdaten enthält das Kapitel 6.1.3.

\subsubsection{Unfalldaten: Erfassung, Aufbereitung und besondere Hinweise für die Auswertung}

Ziel der Datenakquisition war eine nahezu vollständige Erfassung aller polizeilich aufgenommenen Unfälle mit Personenschaden der Jahre 2009 bis 2011 mit Beteiligung von Straßenbahnen. Dafür wurde, je nach Zuständigkeit in den Ländern mit den Innenministerien, den Landespolizeipräsidien und einzelnen Polizeidienststellen der Städte Kontakt aufgenommen.

Bereits vor Beginn des Forschungsvorhabens wurden für die Mehrzahl der Städte die Dienststellen und Ansprechpartner ermittelt, die mit der Aufbereitung und Archivierung der Unfalldaten betraut sind. Im Zuge dessen wurde die grundsätzliche Bereitschaft erfragt, die Unfalldaten für das vorliegende Forschungsvorhaben zur Verfügung stellen zu können. 
Für die Auswertung lagen schlussendlich die Unfalldaten von 58 deutschen Städten in unterschiedlichen Datenformaten vor. Für die Länder Hessen, Thüringen, Berlin und Bremen hat der UDV die Unfalldaten zur Verfügung gestellt.

Für die Städte Saarbrücken und Oberhausen standen die Unfalldaten nicht zur Verfügung, wobei bekannt war, dass sich für Oberhausen lediglich drei Unfälle mit Personenschaden in den Jahren 2009 bis 2011 ereignet hatten.

\section{Zur Verfügung stehende Unfalldaten:}

Tabelle 6-22: Übersicht der vorliegenden polizeilichen Unfalldaten

\begin{tabular}{|l|c|}
\hline \multicolumn{1}{|c|}{ Unfallkategorie } & $\begin{array}{c}\text { Anzahl } \\
\text { Unfälle }\end{array}$ \\
\hline Unfallkategorie 1, Unfall mit Getöteten: & 100 \\
\hline Unfallkategorie 2, Unfall mit Schwerverletzten: & 956 \\
\hline Unfallkategorie 3, Unfall mit Leichtverletzten: & 3043 \\
\hline Unfälle Gesamt: & $\mathbf{4 0 9 9}$ \\
\hline
\end{tabular}

\begin{tabular}{|c|c|c|}
\hline \multicolumn{3}{|c|}{ dabei Verunglückte nach Unfallschwere } \\
\hline $\begin{array}{c}\text { Anzahl } \\
\text { Getötete }\end{array}$ & $\begin{array}{c}\text { Anzahl Schwer- } \\
\text { verletzte }\end{array}$ & $\begin{array}{c}\text { Anzahl Leicht- } \\
\text { verletzte }\end{array}$ \\
\hline 100 & 6 & 58 \\
\hline- & 997 & 497 \\
\hline- & - & 3913 \\
\hline 100 & $\mathbf{1 0 0 3}$ & $\mathbf{4 4 6 8}$ \\
\hline \multicolumn{3}{|c}{} \\
\hline \multicolumn{3}{|c}{} \\
\hline
\end{tabular}

Tabelle 6-23: Übersicht der vorliegenden Unfalldaten nach Unfallfolge und Art der Verkehrsbeteiligung

\begin{tabular}{|c|c|c|c|c|c|}
\hline \multicolumn{2}{|c|}{ Unfallkategorie } & $\begin{array}{l}\text { Unfallkategorie 1, } \\
\text { Unfall mit Getöteten }\end{array}$ & $\begin{array}{l}\text { Unfallkategorie 2, } \\
\text { Unfall mit } \\
\text { Schwerverletzten }\end{array}$ & $\begin{array}{l}\text { Unfallkategorie 3, } \\
\text { Unfall mit } \\
\text { Leichtverletzten }\end{array}$ & Summe \\
\hline \multicolumn{2}{|c|}{$\begin{array}{l}\text { Anzahl Unfälle } \\
\text { bei bekannter Unfallfolge nach Beteiligungsart * }\end{array}$} & 93 & 864 & 2.693 & 3.650 \\
\hline \multirow{6}{*}{$\begin{array}{c}\text { dabei Getötete nach } \\
\text { Art der } \\
\text { Verkehrsbeteiligung }\end{array}$} & Fußgänger & 70 & \multirow{6}{*}{ - } & \multirow{6}{*}{-} & 70 \\
\hline & Radfahrer & 15 & & & 15 \\
\hline & PKW-Insassen & 7 & & & 7 \\
\hline & Straßenbahninsassen & 1 & & & 1 \\
\hline & übrige Verkehrsteilnehmer & 0 & & & 0 \\
\hline & Summe Getötete & 93 & & & 93 \\
\hline \multirow{6}{*}{$\begin{array}{c}\text { dabei } \\
\text { Schwerverletzte } \\
\text { nach Art der } \\
\text { Verkehrsbeteiligung }\end{array}$} & Fußgänger & 2 & 333 & \multirow{6}{*}{-} & 335 \\
\hline & Radfahrer & 0 & 134 & & 134 \\
\hline & PKW-Insassen & 2 & 248 & & 250 \\
\hline & Straßenbahninsassen & 0 & 130 & & 130 \\
\hline & übrige Verkehrsteilnehmer. & 0 & 46 & & 46 \\
\hline & Summe Schwerverletzte & 4 & 891 & & 895 \\
\hline \multirow{6}{*}{$\begin{array}{l}\text { dabei Leichtverletzte } \\
\text { nach Art der } \\
\text { Verkehrsbeteiligung }\end{array}$} & Fußgänger & 0 & 8 & 513 & 521 \\
\hline & Radfahrer & 0 & 3 & 206 & 209 \\
\hline & PKW-Insassen & 3 & 74 & 1.176 & 1.253 \\
\hline & Straßenbahninsassen & 53 & 276 & 1.298 & 1.627 \\
\hline & übrige Verkehrsteilnehmer & 0 & 9 & 174 & 183 \\
\hline & Summe Leichtverletzte & 56 & 370 & 3.367 & 3.793 \\
\hline
\end{tabular}

* Hinweis: Für 449 Unfälle (u.a. die der Städte Braunschweig, Hannover, Ludwigshafen und Mainz) standen die Unfallfolgen differenziert nach den einzelnen Verkehrsbeteiligten nicht zur Verfügung 


\section{Zur Verfügung stehende Unfalldaten nach Datenformat:}

In Abhängigkeit der zur Verfügung stehenden Möglichkeiten, genutzter Software der kooperierenden Dienststellen, liegen die Daten für die 58 Städte in unterschiedlichem Format und auch unterschiedlichem Datenumfang vor.

Grundsätzlich können drei unterschiedliche Datenformate unterschieden werden:

Tabelle 6-24: Dateieigenschaften in Abhängigkeit des Datenformates

\begin{tabular}{|l|l|}
\hline Datenformat & Dateneigenschaften \\
\hline \multirow{3}{*}{ EUSKA $^{100}$ - Format } & - georeferenzierte Unfallstelle, \\
& - alle wesentlichen unfallbeschreibende Attribute liegen vor, \\
& - Unfallhergangsbeschreibungen liegen teilweise vor \\
& - der Aufbau der hinterlegten ACCESS-Datentabellen \\
& unterscheidet sich nicht (einheitliches Datenlayout)
\end{tabular}

Anzahl Unfälle

Unfälle im EUSKA-Format 2240

Unfälle im Excel-Format: 1790

Unfälle als Verkehrsunfallanzeige (VUA): 109 Unfälle Gesamt: 4099

Eine tabellarische Zusammenfassung der erhaltenen Unfalldaten kann Tabelle 6-25 entnommen werden.

\footnotetext{
100 EUSKA: "Elektronische Unfalltypensteckkarte", Softwarelösung zur Auswertung und Archivierung polizeilich erfasster Unfälle; Entwicklung durch die Unfallforschung der Versicherer und PTV AG
} 
Für eine gemeinsame Auswertung der erhaltenen Unfalldaten musste ein einheitliches Datenspeicherformat gefunden werden, in welches alle Unfälle überführt bzw. zusammengeführt werden.

Die Entscheidung fiel hierbei auf das Microsoft Excel-Format ( $\left.{ }^{*} . x \mid s x\right)$ Hierfür wurde zunächst eine standardisierte Tabellenblattaufteilung definiert, die für alle Unfälle maßgeblich war. In den Zeilen untereinander waren die Unfälle gelistet (pro Unfall eine Zeile). In den Spalten wurden die Unfallmerkmale eingepflegt.

\section{Transformation der EXCEL-Daten in das Standard-Tabellenformat:}

Die Transformation der EXCEL-Tabellenblätter in das Standard-Tabellenformat erfolgte durch Formelverknüpfungen. In den Fällen, in denen das Quellformat gleich dem Zielformat war, waren die Formeln reine Querverweise, z.B. bei der bundeseinheitlichen Unfalltypennummer. Häufig war jedoch das Zielformat ungleich dem Zielformat. Als einfaches Beispiel sei der Wochentag genannt: Im Quellformat wurde dieser entweder als ausgeschriebener Wochentag ("Montag"), als verkürzte Angabe ("Mo") oder gar nicht angegeben, sodass der Wochentag über das Unfalldatum ermittelt werden musste. Das Zielformat war eine numerische Größe (Montag = 1, Dienstag =2, ...).

In diesen Fällen musste die Formel zur Transformation den Charakter von logischen Verknüpfungen haben. Aufgrund des in Tabelle 6-24 beschrieben unterschiedlichen Aufbaus und Inhalts der Rohdatentabelle erfolgte für jede Stadt die Aufarbeitung praktisch individuell.

\section{Transformation der EUSKA-Daten in das Standard-Tabellenformat:}

Die Transformation der EUSKA-Daten erfolgte zunächst durch das Kopieren mehrerer Microsoft ACCESS-Datentabellen in mehrere Tabellenblätter einer EXCEL-Datei. Ab da erfolgte die Transformation wie beschrieben von EXCEL-Daten in das Standard-Tabellenformat. Aufgrund des in Tabelle 6-24 beschriebenen einheitlichen Datenlayouts konnten die erstellten Querverweise und logischen Verknüpfungen für alle Städte mit Daten im EUSKA-Format übernommen werden.

\section{Transformation der Verkehrsunfallanzeigen in das Standard-Tabellenformat:}

Die Unfälle der Verkehrsunfallanzeigen wurden zunächst in EUSKA eingepflegt. Unfalltexte und Unfallskizzen wurden nicht nach EUSKA übertragen.

Ab da erfolgte die Transformation wie beschrieben von EUSKA-Daten in das Standard-

Tabellenformat. 
Tabelle 6-25: Datenumfang, Datenformate und Dateninhalte der erhaltenen Unfalldaten

\begin{tabular}{|c|c|c|c|c|c|c|c|c|c|c|c|c|c|c|c|c|c|c|c|c|c|c|c|}
\hline \multirow[b]{3}{*}{ 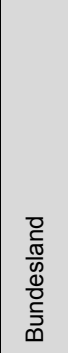 } & \multirow[b]{3}{*}{ 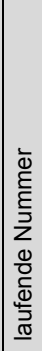 } & \multirow[b]{3}{*}{ Ort } & \multirow[b]{3}{*}{$\begin{array}{l}\text { Roh- } \\
\text { Daten- } \\
\text { format }\end{array}$} & \multicolumn{20}{|c|}{ Rohdaten } \\
\hline & & & & \multicolumn{3}{|c|}{ Zeitraum } & \multicolumn{6}{|c|}{ Anzahl Unfälle } & \multicolumn{11}{|c|}{ Verfügbare Unfallmerkmale } \\
\hline & & & & 尺े & 은 & זั & 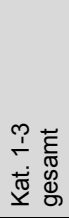 & 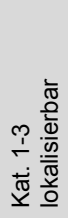 & 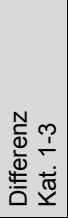 & 㐫 & $\begin{array}{l}\sim \\
\dot{\mathbb{N}} \\
\underline{x}\end{array}$ & $\begin{array}{l}m \\
\dot{\pi} \\
\check{\pi}\end{array}$ & $\frac{\varepsilon}{\sum_{\overparen{D}}}$ & 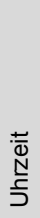 & 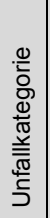 & 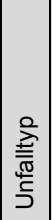 & 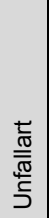 & 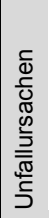 & 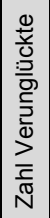 & 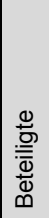 & 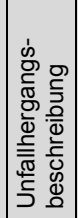 & 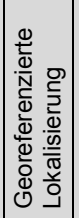 & 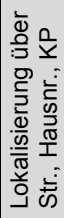 \\
\hline \multirow{7}{*}{ 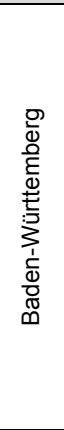 } & 1 & \begin{tabular}{|l|} 
Freiburg im \\
Breisgau \\
\end{tabular} & EUSKA & $\checkmark$ & $\checkmark$ & $\checkmark$ & 75 & 75 & 0 & 3 & 20 & 52 & $\checkmark$ & $\checkmark$ & $\checkmark$ & $\checkmark$ & $\checkmark$ & $\checkmark$ & $\checkmark$ & $\checkmark$ & \begin{tabular}{|c|}
$a b$ \\
2010 \\
\end{tabular} & $\checkmark$ & \\
\hline & 2 & Heilbronn & EUSKA & $\checkmark$ & $\checkmark$ & $\checkmark$ & 7 & 7 & 0 & 0 & 2 & 5 & $\checkmark$ & $\checkmark$ & $\checkmark$ & $\checkmark$ & $\checkmark$ & $\checkmark$ & $\checkmark$ & $\checkmark$ & \begin{tabular}{|c|}
$a b$ \\
2010
\end{tabular} & $\checkmark$ & \\
\hline & 3 & Karlsruhe & EUSKA & $\checkmark$ & $\checkmark$ & $\checkmark$ & 192 & 192 & 0 & 3 & 52 & 137 & $\checkmark$ & $\checkmark$ & $\checkmark$ & $\checkmark$ & $\checkmark$ & $\checkmark$ & $\checkmark$ & $\checkmark$ & \begin{tabular}{|c|}
$a b$ \\
2010
\end{tabular} & $\checkmark$ & \\
\hline & 4 & Heidelberg & EUSKA & $\checkmark$ & $\checkmark$ & $\checkmark$ & 44 & 44 & 0 & 1 & 9 & 34 & $\checkmark$ & $\checkmark$ & $\checkmark$ & $\checkmark$ & $\checkmark$ & $\checkmark$ & $\checkmark$ & $\checkmark$ & $\begin{array}{c}a b \\
2010\end{array}$ & $\checkmark$ & \\
\hline & 5 & Mannheim & EUSKA & $\checkmark$ & $\checkmark$ & $\checkmark$ & 100 & 99 & 1 & 5 & 27 & 68 & $\checkmark$ & $\checkmark$ & $\checkmark$ & $\checkmark$ & $\checkmark$ & $\checkmark$ & $\checkmark$ & $\checkmark$ & $\begin{array}{c}a b \\
2010\end{array}$ & $\checkmark$ & \\
\hline & 6 & Stuttgart & EUSKA & $\checkmark$ & $\checkmark$ & $\checkmark$ & 121 & 121 & 0 & 6 & 37 & 78 & $\checkmark$ & $\checkmark$ & $\checkmark$ & $\checkmark$ & $\checkmark$ & $\checkmark$ & $\checkmark$ & $\checkmark$ & \begin{tabular}{|c|}
$a b$ \\
2010 \\
\end{tabular} & $\checkmark$ & \\
\hline & 7 & Ulm & EUSKA & $\checkmark$ & $\checkmark$ & $\checkmark$ & 6 & 6 & 0 & 0 & 2 & 4 & $\checkmark$ & $\checkmark$ & $\checkmark$ & $\checkmark$ & $\checkmark$ & $\checkmark$ & $\checkmark$ & $\checkmark$ & $\begin{array}{c}a b \\
2010\end{array}$ & $\checkmark$ & \\
\hline \multirow{4}{*}{ 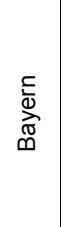 } & 8 & Augsburg & Excel & $\checkmark$ & $\checkmark$ & $\checkmark$ & 99 & 99 & 0 & 1 & 19 & 79 & $\checkmark$ & $\checkmark$ & $\checkmark$ & $\checkmark$ & $\checkmark$ & $\checkmark$ & $\checkmark$ & $\checkmark$ & $x$ & & $\checkmark$ \\
\hline & 9 & München & Excel & $\checkmark$ & $\checkmark$ & $\checkmark$ & 206 & 205 & 1 & 0 & 33 & 173 & $\checkmark$ & $\checkmark$ & $\checkmark$ & $\checkmark$ & $\checkmark$ & $\checkmark$ & $\checkmark$ & $\checkmark$ & $x$ & & $\checkmark$ \\
\hline & 10 & Nürnberg & Excel & $\checkmark$ & $\checkmark$ & $\checkmark$ & 101 & 101 & 0 & 1 & 16 & 84 & $\checkmark$ & $\checkmark$ & $\checkmark$ & $\checkmark$ & $\checkmark$ & $\checkmark$ & $\checkmark$ & $\checkmark$ & $x$ & & $\checkmark$ \\
\hline & 11 & Würzburg & Excel & $\checkmark$ & $\checkmark$ & $\checkmark$ & 33 & 33 & 0 & 1 & 8 & 24 & $\checkmark$ & $\checkmark$ & $\checkmark$ & $\checkmark$ & $\checkmark$ & $\checkmark$ & $\checkmark$ & $\checkmark$ & $x$ & & $\checkmark$ \\
\hline \multirow{8}{*}{ 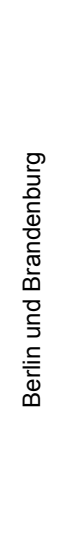 } & 12 & Berlin & EUSKA & $\checkmark$ & $\checkmark$ & $\checkmark$ & 295 & 295 & 0 & 10 & 76 & 209 & $\checkmark$ & $\checkmark$ & $\checkmark$ & $\checkmark$ & $\checkmark$ & $\checkmark$ & $\checkmark$ & $\checkmark$ & $x$ & $\checkmark$ & \\
\hline & 13 & Potsdam & EUSKA & $x$ & $\checkmark$ & $\checkmark$ & 32 & 32 & 0 & 0 & 8 & 24 & $\checkmark$ & $\checkmark$ & $\checkmark$ & $\checkmark$ & $\checkmark$ & $\checkmark$ & $\checkmark$ & $\checkmark$ & $\checkmark$ & $\checkmark$ & \\
\hline & 14 & \begin{tabular}{|l|} 
Schöneiche/ \\
Rüdersdorf \\
(LK Oder-Spree) \\
\end{tabular} & EUSKA & $x$ & $\checkmark$ & $\checkmark$ & 7 & 7 & 0 & 1 & 2 & 4 & $\checkmark$ & $\checkmark$ & $\checkmark$ & $\checkmark$ & $\checkmark$ & $\checkmark$ & $\checkmark$ & $\checkmark$ & $\checkmark$ & $\checkmark$ & \\
\hline & 15 & $\begin{array}{l}\text { Woltersdorf } \\
\text { (zu Schöneiche/ } \\
\text { Rüdersdorf) }\end{array}$ & EUSKA & $x$ & $\checkmark$ & $\checkmark$ & 0 & 0 & 0 & 0 & 0 & 0 & $\checkmark$ & $\checkmark$ & $\checkmark$ & $\checkmark$ & $\checkmark$ & $\checkmark$ & $\checkmark$ & $\checkmark$ & $\checkmark$ & $\checkmark$ & \\
\hline & 16 & $\begin{array}{l}\text { Strausberg } \\
\text { (LK Märkisch } \\
\text { Oderland) }\end{array}$ & EUSKA & $x$ & $\checkmark$ & $\checkmark$ & 0 & 0 & 0 & 0 & 0 & 0 & $\checkmark$ & $\checkmark$ & $\checkmark$ & $\checkmark$ & $\checkmark$ & $\checkmark$ & $\checkmark$ & $\checkmark$ & $\checkmark$ & $\checkmark$ & \\
\hline & 17 & \begin{tabular}{|l|}
$\begin{array}{l}\text { Brandenburg } \\
\text { (Havel) }\end{array}$ \\
\end{tabular} & EUSKA & $x$ & $\checkmark$ & $\checkmark$ & 3 & 3 & 0 & 0 & 0 & 3 & $\checkmark$ & $\checkmark$ & $\checkmark$ & $\checkmark$ & $\checkmark$ & $\checkmark$ & $\checkmark$ & $\checkmark$ & $\checkmark$ & $\checkmark$ & \\
\hline & 18 & Cottbus & EUSKA & $x$ & $\checkmark$ & $\checkmark$ & 6 & 6 & 0 & 1 & 1 & 4 & $\checkmark$ & $\checkmark$ & $\checkmark$ & $\checkmark$ & $\checkmark$ & $\checkmark$ & $\checkmark$ & $\checkmark$ & $\checkmark$ & $\checkmark$ & \\
\hline & 19 & Frankfurt (Oder) & EUSKA & $x$ & $\checkmark$ & $\checkmark$ & 5 & 5 & 0 & 1 & 2 & 2 & $\checkmark$ & $\checkmark$ & $\checkmark$ & $\checkmark$ & $\checkmark$ & $\checkmark$ & $\checkmark$ & $\checkmark$ & $\checkmark$ & $\checkmark$ & \\
\hline \multirow{3}{*}{$\begin{array}{l}\text { ঠ } \\
\mathscr{D} \\
\stackrel{1}{I}\end{array}$} & 20 & Darmstadt & EUSKA & $\checkmark$ & $\checkmark$ & $\checkmark$ & 56 & 56 & 0 & 3 & 10 & 43 & $\checkmark$ & $\checkmark$ & $\checkmark$ & $\checkmark$ & $\checkmark$ & $\checkmark$ & $\checkmark$ & $\checkmark$ & $x$ & $\checkmark$ & \\
\hline & 21 & $\begin{array}{l}\text { Frankfurt am } \\
\text { Main } \\
\end{array}$ & EUSKA & $\checkmark$ & $\checkmark$ & $\checkmark$ & 231 & 229 & 2 & 9 & 44 & 178 & $\checkmark$ & $\checkmark$ & $\checkmark$ & $\checkmark$ & $\checkmark$ & $\checkmark$ & $\checkmark$ & $\checkmark$ & $x$ & $\checkmark$ & \\
\hline & 22 & Kassel & EUSKA & $\checkmark$ & $\checkmark$ & $\checkmark$ & 80 & 80 & 0 & 1 & 13 & 66 & $\checkmark$ & $\checkmark$ & $\checkmark$ & $\checkmark$ & $\checkmark$ & $\checkmark$ & $\checkmark$ & $\checkmark$ & $x$ & $\checkmark$ & \\
\hline \multirow{2}{*}{ 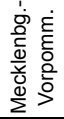 } & 23 & Rostock & VUA & $\checkmark$ & $\checkmark$ & $\checkmark$ & 46 & 46 & 0 & 0 & 14 & 32 & $\checkmark$ & $\checkmark$ & $\checkmark$ & $\checkmark$ & $\checkmark$ & $\checkmark$ & $\checkmark$ & $\checkmark$ & $\checkmark$ & & $\checkmark$ \\
\hline & 24 & Schwerin & VUA & $\checkmark$ & $\checkmark$ & $\checkmark$ & 11 & 11 & 0 & 0 & 4 & 7 & $\checkmark$ & $\checkmark$ & $\checkmark$ & $\checkmark$ & $\checkmark$ & $\checkmark$ & $\checkmark$ & $\checkmark$ & $\checkmark$ & & $\checkmark$ \\
\hline \multirow{3}{*}{ 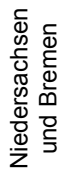 } & 25 & Braunschweig & Excel & $\checkmark$ & $\checkmark$ & $\checkmark$ & 36 & 33 & 3 & 1 & 14 & 21 & $\checkmark$ & $\checkmark$ & $\checkmark$ & $\checkmark$ & $\checkmark$ & $\checkmark$ & $\checkmark$ & $\checkmark$ & $\checkmark$ & & $\checkmark$ \\
\hline & 26 & Hannover & Excel & $\checkmark$ & $\checkmark$ & $\checkmark$ & 178 & 149 & 29 & 6 & 47 & 125 & $\checkmark$ & $\checkmark$ & $\checkmark$ & $\checkmark$ & $\checkmark$ & $\checkmark$ & $\checkmark$ & $\checkmark$ & $\checkmark$ & & $\checkmark$ \\
\hline & 27 & Bremen & EUSKA & $\checkmark$ & $\checkmark$ & $\checkmark$ & 173 & 163 & 10 & 2 & 29 & 142 & $\checkmark$ & $\checkmark$ & $\checkmark$ & $\checkmark$ & $\checkmark$ & $\checkmark$ & $\checkmark$ & $\checkmark$ & $x$ & $\checkmark$ & \\
\hline \multirow{6}{*}{ 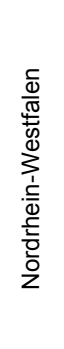 } & 28 & Bielefeld & Excel & $\checkmark$ & $\checkmark$ & $\checkmark$ & 32 & 30 & 2 & 0 & 9 & 23 & $\checkmark$ & $\checkmark$ & $\checkmark$ & $\checkmark$ & $\checkmark$ & $\checkmark$ & $\checkmark$ & $\checkmark$ & $x$ & & $\checkmark$ \\
\hline & 29 & Bochum & Excel & $\checkmark$ & $\checkmark$ & $\checkmark$ & 37 & 32 & 5 & 1 & 5 & 31 & $\checkmark$ & $\checkmark$ & $\checkmark$ & $\checkmark$ & $\checkmark$ & $\checkmark$ & $\checkmark$ & $\checkmark$ & $x$ & & $\checkmark$ \\
\hline & 30 & Gelsenkirchen & Excel & $\checkmark$ & $\checkmark$ & $\checkmark$ & 23 & 14 & 9 & 2 & 7 & 14 & $\checkmark$ & $\checkmark$ & $\checkmark$ & $\checkmark$ & $\checkmark$ & $\checkmark$ & $\checkmark$ & $\checkmark$ & $x$ & & $\checkmark$ \\
\hline & 31 & Bonn & Excel & $\checkmark$ & $\checkmark$ & $\checkmark$ & 47 & 40 & 7 & 0 & 13 & 34 & $\checkmark$ & $\checkmark$ & $\checkmark$ & $\checkmark$ & $\checkmark$ & $\checkmark$ & $\checkmark$ & $\checkmark$ & $x$ & & $\checkmark$ \\
\hline & 32 & Dortmund & Excel & $\checkmark$ & $\checkmark$ & $\checkmark$ & 76 & 71 & 5 & 1 & 15 & 60 & $\checkmark$ & $\checkmark$ & $\checkmark$ & $\checkmark$ & $\checkmark$ & $\checkmark$ & $\checkmark$ & $\checkmark$ & $x$ & & $\checkmark$ \\
\hline & 33 & Duisburg & Excel & $\checkmark$ & $\checkmark$ & $\checkmark$ & 46 & 30 & 16 & 1 & 4 & 41 & $\checkmark$ & $\checkmark$ & $\checkmark$ & $\checkmark$ & $\checkmark$ & $\checkmark$ & $\checkmark$ & $\checkmark$ & $x$ & & $\checkmark$ \\
\hline
\end{tabular}




\begin{tabular}{|c|c|c|c|c|c|c|c|c|c|c|c|c|c|c|c|c|c|c|c|c|c|c|c|}
\hline \multirow[b]{3}{*}{ 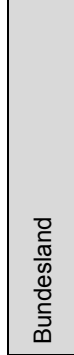 } & \multirow[b]{3}{*}{ 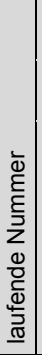 } & \multirow[b]{3}{*}{ Ort } & \multirow[b]{3}{*}{$\begin{array}{l}\text { Roh- } \\
\text { Daten- } \\
\text { format }\end{array}$} & \multicolumn{20}{|c|}{ Rohdaten } \\
\hline & & & & \multicolumn{3}{|c|}{ Zeitraum } & \multicolumn{6}{|c|}{ Anzahl Unfälle } & \multicolumn{11}{|c|}{ Verfügbare Unfallmerkmale } \\
\hline & & & & ¿्.े & 울 & $\bar{i}$ & 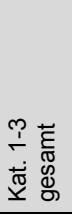 & 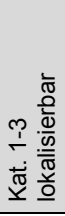 & 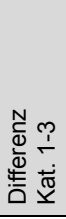 & 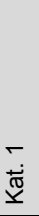 & 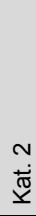 & $\begin{array}{l}m \\
\dot{\mathbb{\pi}} \\
\stackrel{1}{\underline{y}}\end{array}$ & 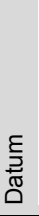 & 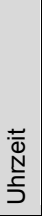 & 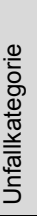 & 胥 & 売 & 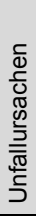 & 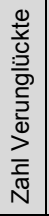 & 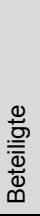 & 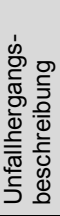 & 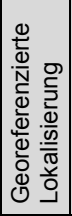 & 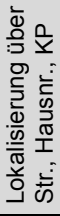 \\
\hline & 34 & Düsseldorf & Excel & $\checkmark$ & $\checkmark$ & $\checkmark$ & 252 & 228 & 24 & 9 & 57 & 186 & $\checkmark$ & $\checkmark$ & $\checkmark$ & $\checkmark$ & $\checkmark$ & $\checkmark$ & $\checkmark$ & $\checkmark$ & $\mathrm{x}$ & & $\checkmark$ \\
\hline & 35 & Essen & Excel & $\checkmark$ & $\checkmark$ & $\checkmark$ & 102 & 77 & 25 & 2 & 20 & 80 & $\checkmark$ & $\checkmark$ & $\checkmark$ & $\checkmark$ & $\checkmark$ & $\checkmark$ & $\checkmark$ & $\checkmark$ & $x$ & & $\checkmark$ \\
\hline & 36 & Köln & Excel & $\checkmark$ & $\checkmark$ & $\checkmark$ & 277 & 229 & 48 & 10 & 84 & 183 & $\checkmark$ & $\checkmark$ & $\checkmark$ & $\checkmark$ & $\checkmark$ & $\checkmark$ & $\checkmark$ & $\checkmark$ & $\mathrm{x}$ & & $\checkmark$ \\
\hline & 37 & Krefeld & Excel & $\checkmark$ & $\checkmark$ & $\checkmark$ & 37 & 29 & 8 & 0 & 5 & 32 & $\checkmark$ & $\checkmark$ & $\checkmark$ & $\checkmark$ & $\checkmark$ & $\checkmark$ & $\checkmark$ & $\checkmark$ & $\mathrm{x}$ & & $\checkmark$ \\
\hline & 38 & $\begin{array}{l}\text { Mülheim (Ruhr) } \\
\text { (zu Essen) }\end{array}$ & Excel & $\checkmark$ & $\checkmark$ & $\checkmark$ & 0 & 0 & 0 & 0 & 0 & 0 & $\checkmark$ & $\checkmark$ & $\checkmark$ & $\checkmark$ & $\checkmark$ & $\checkmark$ & $\checkmark$ & $\checkmark$ & $x$ & & $\checkmark$ \\
\hline & 39 & Oberhausen & Excel & $\mathrm{x}$ & $\mathrm{x}$ & $\mathrm{x}$ & 0 & 0 & 0 & 0 & 0 & 0 & $\mathrm{x}$ & $x$ & $x$ & $x$ & $x$ & $x$ & $x$ & $\mathrm{x}$ & $x$ & $x$ & $\mathrm{x}$ \\
\hline \multirow{3}{*}{ 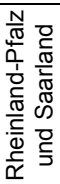 } & 40 & $\begin{array}{l}\text { Ludwigshafen } \\
\text { am Rhein }\end{array}$ & Excel & $\checkmark$ & $\checkmark$ & $\checkmark$ & 44 & 41 & 3 & 0 & 9 & 35 & $\checkmark$ & $\checkmark$ & $\checkmark$ & $\checkmark$ & $x$ & $\checkmark$ & $\checkmark$ & $\checkmark$ & $x$ & & $\checkmark$ \\
\hline & 41 & Mainz & Excel & $\checkmark$ & $\checkmark$ & $\checkmark$ & 29 & 29 & 0 & 0 & 6 & 23 & $\checkmark$ & $\checkmark$ & $\checkmark$ & $\checkmark$ & $x$ & $\checkmark$ & $\checkmark$ & $\checkmark$ & $\checkmark$ & & $\checkmark$ \\
\hline & 42 & Saarbrücken & $\mathrm{x}$ & $\mathrm{x}$ & $\mathrm{x}$ & $x$ & 0 & 0 & 0 & 0 & 0 & 0 & $\mathrm{x}$ & $\mathrm{x}$ & $\mathrm{x}$ & $\mathrm{x}$ & $\mathrm{x}$ & $\mathrm{x}$ & $\mathrm{x}$ & $\mathrm{x}$ & $x$ & $\mathrm{x}$ & $x$ \\
\hline \multirow{7}{*}{ 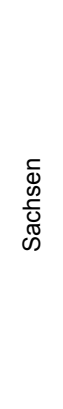 } & 43 & Dresden & EUSKA & $\checkmark$ & $\checkmark$ & $\checkmark$ & 227 & 227 & 0 & 2 & 58 & 167 & $\checkmark$ & $\checkmark$ & $\checkmark$ & $\checkmark$ & $\checkmark$ & $\checkmark$ & $\checkmark$ & $\checkmark$ & $\checkmark$ & $\checkmark$ & \\
\hline & 44 & Bad Schandau & EUSKA & $\checkmark$ & $\checkmark$ & $\checkmark$ & 0 & 0 & 0 & 0 & 0 & 0 & $\checkmark$ & $\checkmark$ & $\checkmark$ & $\checkmark$ & $\checkmark$ & $\checkmark$ & $\checkmark$ & $\checkmark$ & $\checkmark$ & $\checkmark$ & \\
\hline & 45 & Chemnitz & EUSKA & $\checkmark$ & $\checkmark$ & $\checkmark$ & 68 & 68 & 0 & 3 & 13 & 52 & $\checkmark$ & $\checkmark$ & $\checkmark$ & $\checkmark$ & $\checkmark$ & $\checkmark$ & $\checkmark$ & $\checkmark$ & $\checkmark$ & $\checkmark$ & \\
\hline & 46 & Görlitz & EUSKA & $\checkmark$ & $\checkmark$ & $\checkmark$ & 5 & 5 & 0 & 0 & 1 & 4 & $\checkmark$ & $\checkmark$ & $\checkmark$ & $\checkmark$ & $\checkmark$ & $\checkmark$ & $\checkmark$ & $\checkmark$ & $\checkmark$ & $\checkmark$ & \\
\hline & 47 & Leipzig & EUSKA & $\checkmark$ & $\checkmark$ & $\checkmark$ & 190 & 190 & 0 & 6 & 52 & 132 & $\checkmark$ & $\checkmark$ & $\checkmark$ & $\checkmark$ & $\checkmark$ & $\checkmark$ & $\checkmark$ & $\checkmark$ & $\checkmark$ & $\checkmark$ & \\
\hline & 48 & Plauen & EUSKA & $\checkmark$ & $\checkmark$ & $\checkmark$ & 12 & 12 & 0 & 0 & 4 & 8 & $\checkmark$ & $\checkmark$ & $\checkmark$ & $\checkmark$ & $\checkmark$ & $\checkmark$ & $\checkmark$ & $\checkmark$ & $\checkmark$ & $\checkmark$ & \\
\hline & 49 & Zwickau & EUSKA & $\checkmark$ & $\checkmark$ & $\checkmark$ & 32 & 32 & 0 & 0 & 10 & 22 & $\checkmark$ & $\checkmark$ & $\checkmark$ & $\checkmark$ & $\checkmark$ & $\checkmark$ & $\checkmark$ & $\checkmark$ & $\checkmark$ & $\checkmark$ & \\
\hline \multirow{6}{*}{ 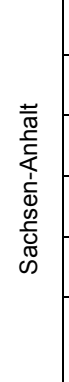 } & 50 & Dessau-Roßlau & EUSKA & $\checkmark$ & $\checkmark$ & $\checkmark$ & 5 & 5 & 0 & 0 & 2 & 3 & $\checkmark$ & $\checkmark$ & $\checkmark$ & $\checkmark$ & $\checkmark$ & $\checkmark$ & $\checkmark$ & $\checkmark$ & $\checkmark$ & $\checkmark$ & \\
\hline & 51 & Halberstadt & EUSKA & $\checkmark$ & $\checkmark$ & $\checkmark$ & 3 & 3 & 0 & 0 & 0 & 3 & $\checkmark$ & $\checkmark$ & $\checkmark$ & $\checkmark$ & $\checkmark$ & $\checkmark$ & $\checkmark$ & $\checkmark$ & $\checkmark$ & $\checkmark$ & \\
\hline & 52 & $\begin{array}{l}\text { Halle an der } \\
\text { Saale }\end{array}$ & EUSKA & $\checkmark$ & $\checkmark$ & $\checkmark$ & 135 & 135 & 0 & 3 & 33 & 99 & $\checkmark$ & $\checkmark$ & $\checkmark$ & $\checkmark$ & $\checkmark$ & $\checkmark$ & $\checkmark$ & $\checkmark$ & $\checkmark$ & $\checkmark$ & \\
\hline & 53 & Magdeburg & EUSKA & $\checkmark$ & $\checkmark$ & $\checkmark$ & 123 & 123 & 0 & 2 & 34 & 87 & $\checkmark$ & $\checkmark$ & $\checkmark$ & $\checkmark$ & $\checkmark$ & $\checkmark$ & $\checkmark$ & $\checkmark$ & $\checkmark$ & $\checkmark$ & \\
\hline & 54 & Merseburg & EUSKA & $\checkmark$ & $\checkmark$ & $\checkmark$ & 7 & 7 & 0 & 0 & 0 & 7 & $\checkmark$ & $\checkmark$ & $\checkmark$ & $\checkmark$ & $\checkmark$ & $\checkmark$ & $\checkmark$ & $\checkmark$ & $\checkmark$ & $\checkmark$ & \\
\hline & 55 & \begin{tabular}{|l|} 
Naumburg \\
(Saale) \\
(Burgenlandkreis)
\end{tabular} & EUSKA & $\checkmark$ & $\checkmark$ & $\checkmark$ & 0 & 0 & 0 & 0 & 0 & 0 & $\checkmark$ & $\checkmark$ & $\checkmark$ & $\checkmark$ & $\checkmark$ & $\checkmark$ & $\checkmark$ & $\checkmark$ & $\checkmark$ & $\checkmark$ & \\
\hline \multirow{5}{*}{ 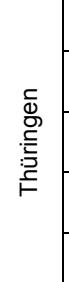 } & 56 & Erfurt & Excel & $\checkmark$ & $\checkmark$ & $\checkmark$ & 58 & 58 & 0 & 1 & 11 & 46 & $\checkmark$ & $\checkmark$ & $\checkmark$ & $\checkmark$ & $\checkmark$ & $\checkmark$ & $\checkmark$ & $\checkmark$ & $\checkmark$ & & $\checkmark$ \\
\hline & 57 & Gera & VUA & $\checkmark$ & $\checkmark$ & $\checkmark$ & 39 & 39 & 0 & 1 & 8 & 30 & $\checkmark$ & $\checkmark$ & $\checkmark$ & $\checkmark$ & $\checkmark$ & $\checkmark$ & $\checkmark$ & $\checkmark$ & $\checkmark$ & & $\checkmark$ \\
\hline & 58 & $\begin{array}{l}\text { Gotha/ } \\
\text { Waltershausen }\end{array}$ & VUA & $\checkmark$ & $\checkmark$ & $\checkmark$ & 4 & 4 & 0 & 0 & 1 & 3 & $\checkmark$ & $\checkmark$ & $\checkmark$ & $\checkmark$ & $\checkmark$ & $\checkmark$ & $\checkmark$ & $\checkmark$ & $\checkmark$ & & $\checkmark$ \\
\hline & 59 & Jena & Excel & $\checkmark$ & $\checkmark$ & $\checkmark$ & 37 & 37 & 0 & 0 & 5 & 32 & $\checkmark$ & $\checkmark$ & $\checkmark$ & $\checkmark$ & $\checkmark$ & $\checkmark$ & $\checkmark$ & $\checkmark$ & $\checkmark$ & & $\checkmark$ \\
\hline & 60 & Nordhausen & VUA & $\checkmark$ & $\checkmark$ & $\checkmark$ & 9 & 9 & 0 & 0 & 1 & 8 & $\checkmark$ & $\checkmark$ & $\checkmark$ & $\checkmark$ & $\checkmark$ & $\checkmark$ & $\checkmark$ & $\checkmark$ & $\checkmark$ & & $\checkmark$ \\
\hline
\end{tabular}

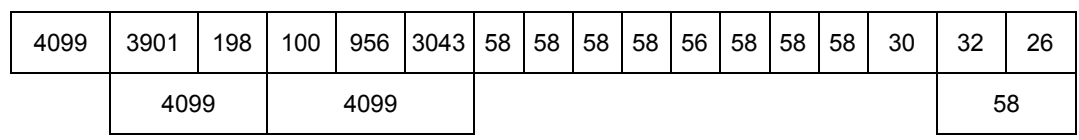




\subsubsection{Netzdaten und straßenräumliche Merkmale: Erfassung, Aufbereitung und besondere Hinweise für die Auswertung}

\section{Verkehrsstärken der Straßenbahn}

Für Auswertungen nach fahrleistungsbezogenen Kenngrößen von Straßenbahnunfällen wurden diese in Bezug zur Straßenbahnverkehrsstärke gesetzt. Die Anzahl der Straßenbahnen je $24 \mathrm{~h}$ sowie über verschiedene Tageszeiträume im Querschnitt mussten dafür vorab erhoben werden (vgl. Anhang A.7.1).

Die Ermittlung der Querschnittsverkehrsstärken geschah über die Auswertung von Fahrplandaten, welche für alle vorliegenden Straßenbahnnetze dem Internet entnommen werden konnten. Vorzugsweise wurden dabei Fahrplandaten entsprechend der Unfalljahre 2009 bis $2011 \mathrm{zu}$ Grunde gelegt. Im Wesentlichen handelte es sich jedoch um Fahrplandaten aus dem Jahren 2013 oder 2014.

Ausgewertet wurden mittlere Wochentage außerhalb der Ferien.

Die Ermittlung der Querschnittsdaten erfolgte mittels eines Tabellenkalkulationsprogramms (LibreOffice 3.6) abschnittsfein zwischen Gabelpunkten von Linien bzw. End- und Wendehaltestellen.

An Beginn und Ende von Straßenbahnlinien bzw. an Gleisschleifen wurden die Linienverkehrsstärken ermittelt. An Gabelpunkten von Linien wurden entsprechend die Linienverkehrsstärken miteinander addiert, sodass die abschnittfeinen Verkehrsmengen auslesbar waren (s. Abbildung 6-20).

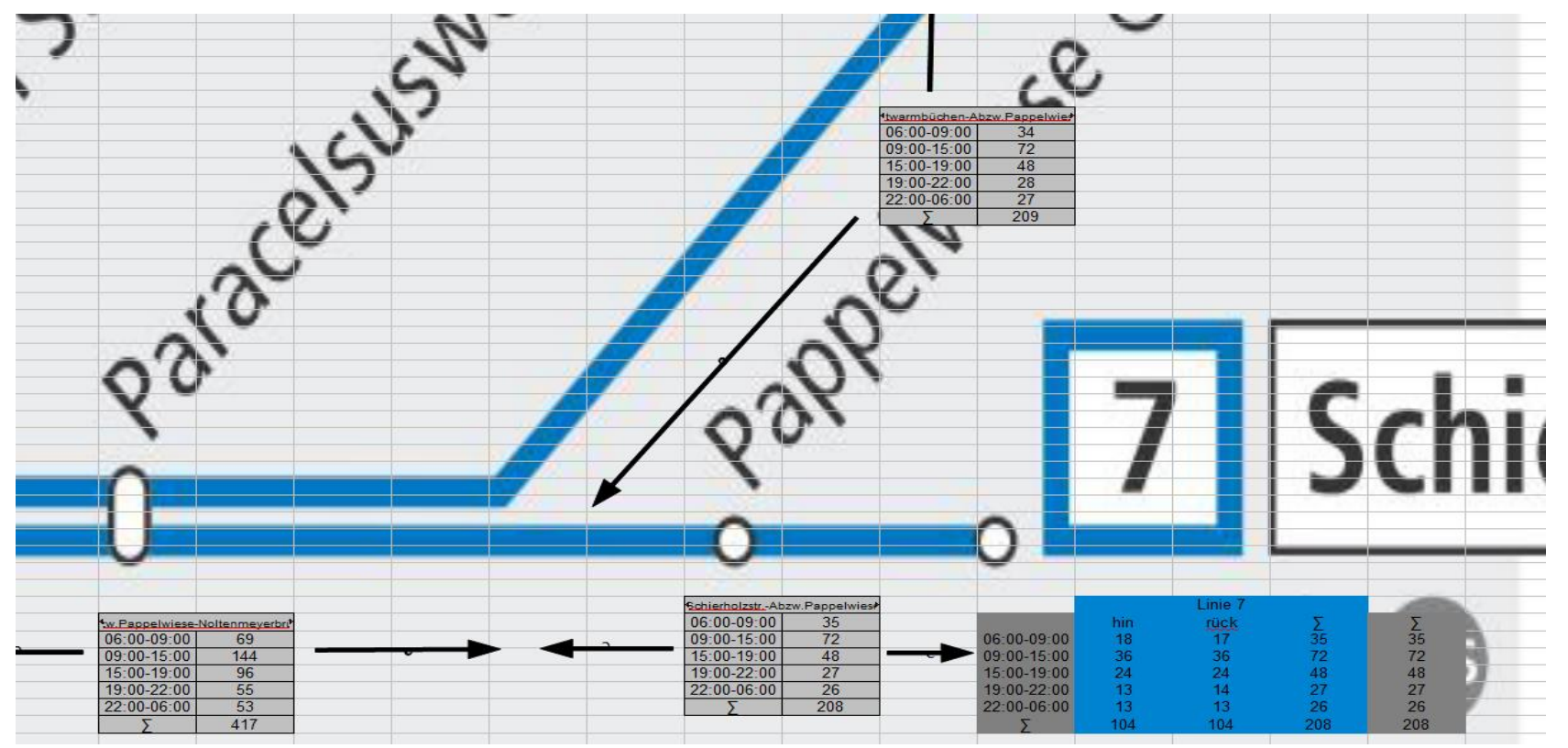

Abbildung 6-20: Ermittlung der abschnittsfeinen Straßenbahnquerschnittsbelastung am Beispiel Hannover

Allen lokalisierbaren Unfällen in der Excel-Unfalldatengesamtliste wurden entsprechend des Straßenabschnitts eine ermittelte Gesamttagesstraßenbahnverkehrsstärke sowie Verkehrsstärken der Stundengruppen 06.00 - 09.00 Uhr, 09.00 - 15.00 Uhr, 15.00 -19.00 Uhr, 19.00 - 22.00 Uhr und 22.00 Uhr zugeordnet. 


\section{Verkehrsstärken des Kraftfahrzeug-Verkehrs}

Für die mikroskopische Analyse (Kapitel 7) wurden zur Einordnung der näher untersuchten Verkehrsanlagen die (durchschnittlich) täglichen Verkehrsstärken (DTV $\mathrm{Kfz}_{\mathrm{z}}$ ) in den Dossierbriefen angegeben. Im Wesentlichen beruhen diese Werte auf Zähldaten, welche durch die Kommunen im Rahmen des Forschungsvorhabens zur Verfügung gestellt wurden. In Abhängigkeit der vorliegenden Daten und der Art der Erhebungsmethodik ist der Erhebungszeitraum der Kraftfahrzeugverkehrsmengendaten sehr unterschiedlich.

Häufig waren händisch erhobene Zähldaten aus Kurzzeitzählungen die Basis für die ermittelten Tagesverkehrsstärken. In selteneren Fällen waren automatisch erhobene Zählwerte an Dauerzählstellen von z.B. verkehrsabhäng gesteuerten Lichtsignalanlagen oder Werte aus Verkehrsmodellen der Kommunen Grundlage der täglichen Verkehrsstärke.

Für Unfallstellen der mikroskopischen Analyse, für die keine Zähldaten zur Verfügung standen, wurden eigene Kurzzeitzählungen nach $\mathrm{HBS}^{101}$ durchgeführt und auf die durchschnittlich tägliche Verkehrsstärke hochgerechnet.

In Tabelle 7-29 (Kapitel 7.1, S. 97) sind die Werte der täglichen Verkehrsstärke für die jeweiligen auffälligen Unfallstellen der mikroskopischen Untersuchung, der Erhebungszeitraum sowie die Datenquelle der Werte enthalten.

\section{Straßenräumliche Merkmale}

Neben den polizeilich erfassten Merkmalen der vorliegenden Unfalldaten werden den lokalisierten Unfällen weitere verkehrliche und straßenräumliche Merkmale für die anschließende Bewertung der Straßeninfrastruktur zugeordnet. Die Erfassung erfolgte im Wesentlichen aus Luftbildern und Befahrungsbildern der Kartendienste Google maps ${ }^{102}$ und Google earth ${ }^{103}$ und Luftbildern des Kartendienstes Bing Maps ${ }^{104}$.

Im folgenden Absatz ist die Gesamtheit der straßenräumlichen und verkehrlichen Merkmale aufgelistet.

\section{Zusammenfassung der Merkmale für die Typisierung untersuchter Verkehrsanlagen}

Im Folgenden werden die weiteren erhobenen Merkmale mit deren möglichen

Merkmalsausprägungen dargestellt. Dabei wird unterschieden, ob die Daten im Rahmen des AP 3 für die Gesamtheit der vorliegenden Unfälle mit Personenschaden (Makroanalyse) und/ oder im Rahmen des AP 5 bei der Detailanalyse ausgewählter Knotenpunkte und Streckenabschnitte (Mikroanalyse) erhoben wurden.

Tabelle 6-26: Differenzierungsmerkmale der Unfälle bei der Makro- und Mikroanalyse

\begin{tabular}{|l|c|c|}
\hline Differenzierungsmerkmal & $\begin{array}{c}\text { Makro- } \\
\text { analyse }\end{array}$ & $\begin{array}{c}\text { Mikro- } \\
\text { analyse }\end{array}$ \\
\hline 1. Verkehrsstärke der Straßenbahn (nach Stundengruppe) & $\mathrm{x}$ \\
\hline 2. Verkehrsstärke der Kfz-Verkehrs (nach Stundengruppe) & $\mathrm{x}$ \\
\hline
\end{tabular}

\footnotetext{
101 [HBS 2009]

102 [GOOGLE MAPS 2014]

103 [GOOGLE EARTH 2014]

104 [BING MAPS 2014]
} 


\begin{tabular}{|c|c|c|}
\hline Differenzierungsmerkmal & $\begin{array}{l}\text { Makro- } \\
\text { analyse }\end{array}$ & $\begin{array}{l}\text { Mikro- } \\
\text { analyse }\end{array}$ \\
\hline 3. Entwurfssituation & & \\
\hline \begin{tabular}{l|l} 
3a. Entwurfssituation in Analogie zu den RASt $2006^{105}$ \\
\end{tabular} & $\mathrm{x}$ & \\
\hline 3b. Geschäftsbesatz von Geschäftsstraßen (Anteil des Geschäftsbesatzes in \%) & $\mathrm{x}$ & \\
\hline 4. Querschnittsmerkmale der freien Strecke und Knotenpunktzufahrten & & \\
\hline $\begin{array}{l}\text { 4a. Querschnittstyp } \\
\text { (in Abhängigkeit der Führung der Straßenbahn und Kfz-Fahrtreifenanzahl) }\end{array}$ & $\mathrm{x}$ & \\
\hline 4b. Führung des Radverkehrs im Querschnitt & $\mathrm{x}$ & \\
\hline 4c. Form des Parkens im Querschnitt & $\mathrm{x}$ & \\
\hline 5. zulässige Höchstgeschwindigkeit des Kfz-Verkehrs (in km/ h) & & $\mathrm{x}$ \\
\hline 6. Knotenpunktmerkmale & & \\
\hline \begin{tabular}{l|l} 
6a. Knotenpunktabstand im Vorfeld eines Knotenpunktes \\
\end{tabular} & $\mathrm{x}$ & \\
\hline 6b. Knotenpunktabstand nach einem Knotenpunkt & $\mathrm{x}$ & \\
\hline 6c. Knotenpunkttyp & $\mathrm{x}$ & \\
\hline 6d. Anzahl der Knotenpunktarme & $\mathrm{x}$ & \\
\hline 6e. Anzahl der Fahrstreifen je Knotenpunktarm & $\mathrm{x}$ & \\
\hline $\begin{array}{l}\text { 6f. Querschnittstyp je Knotenpunktarm } \\
\text { (in Abhängigkeit der Führung der Straßenbahn und Kfz-Fahrtreifenanzahl) }\end{array}$ & $\mathrm{x}$ & \\
\hline 7. Haltestellenmerkmale & & \\
\hline \begin{tabular}{l|l} 
7a. Haltestellenform \\
\end{tabular} & $x$ & \\
\hline 7b. Umsteigehaltestelle (ja/ nein) & $\mathrm{x}$ & \\
\hline 8. Merkmale von Querungsstellen und Bahnübergängen & & \\
\hline 8a. Querungsstellenabstand (nach Entfernungsgruppe) & $\mathrm{x}$ & \\
\hline 8b. Form der Querungsanlage & $x$ & \\
\hline
\end{tabular}

Weitergehende Informationen zur Differenzierung der Merkmale und zur Erhebungsmethodik sind im Anhang A.7 nachzulesen.

\section{Unfallkostensätze}

Um Unfallzahl und Unfallschwere zusammenzufassen und vergleichend gegenüberstellen zu können, wurden die Folgen der Straßenbahnunfälle monetarisiert. Dazu wurden entsprechend der ermittelten Verunglücktenstruktur von Straßenbahnunfällen sowie Verunglücktenkostensätzen und anteilige Sachschadenskostensätzen der $\mathrm{BASt}^{106}$ angepasste Unfallkosten für Straßenbahnunfälle mit Personenschaden in Abhängigkeit der Unfallkategorie berechnet.

Die berechneten, angepassten Unfallkostensätze sind wie folgt:

Unfall mit Getöteten (Unfallkategorie 1):

Unfall mit Schwerverletzten (Unfallkategorie 2):

Unfall mit Leichtverletzten (Unfallkategorie 3):
1.096.419 €/ Unfall

$139.441 € /$ Unfall

$18.926 € /$ Unfall

105 [RASt 2006], S. 17

106 [LERNER 2014] 


\begin{tabular}{|c|c|c|c|c|}
\hline & Anz. Absolut & GT & SV & LV \\
\hline $\mathrm{U}(\mathrm{Gt})$ & 100 & 100 & 6 & 58 \\
\hline $\mathrm{U}(\mathrm{SV})$ & 956 & $\mathrm{x}$ & 997 & 497 \\
\hline $\mathrm{U}(\mathrm{LV})$ & 3043 & $\mathrm{x}$ & $x$ & 3913 \\
\hline
\end{tabular}

\begin{tabular}{|c|c|}
\hline \multicolumn{2}{|c|}{ Verunglücktenkostensätze } \\
\hline & $\mathrm{KSV}[€ / \mathrm{V}]$ \\
\hline Gt & $1.044 .808,15$ \\
\hline SV & $112.365,92$ \\
\hline LV & $4.432,65$ \\
\hline
\end{tabular}

\begin{tabular}{|c|c|}
\hline \multicolumn{2}{|c|}{ Sachschadenskostensätze } \\
\hline & $\mathrm{KSS}[€ / \mathrm{U}]$ \\
\hline $\mathrm{U}(\mathrm{Gt})$ & $42.298,26$ \\
\hline U (SV) & $19.951,24$ \\
\hline U (LV) & $13.262,35$ \\
\hline
\end{tabular}

\begin{tabular}{|l|c|c|c|}
\hline Struktur & GT & SV & LV \\
\hline$U(G t)$ & 1,00 & 0,06 & 0,58 \\
\hline$U(S V)$ & $x$ & 1,04 & 0,52 \\
\hline$U(L V)$ & $x$ & $x$ & 1,29 \\
\hline
\end{tabular}

angepasste Unfallkostensätze

\begin{tabular}{|l|r|}
\hline $\mathrm{KSU}(\mathrm{GT})[€ / \mathrm{U}$ & 1.096 .419 \\
\hline $\mathrm{KSU}(\mathrm{SV})[€ / \mathrm{U}]$ & 139.441 \\
\hline $\mathrm{KSU}(\mathrm{LV})[€ / \mathrm{U}]$ & 18.962 \\
\hline
\end{tabular}

Abbildung 6-21: berechnete angepasste Unfallkostensätze

Quelle: $\quad$ eigene Berechnungen auf Basis der nach LERNER 2014 zur Verfügung gestellten

Verunglücktenkostensätze und Sachschadenskostensätze

\subsection{Ergebnisse der makroskopischen Analyse}

Ergebnisse der makroskopischen Analyse sind Diagramme, die aus der Filterung und Darstellung nach Merkmalsverteilungen aus den vorliegenden Unfällen mit Personenschaden unter Beteiligung von Straßenbahnen hervorgehen.

Die Dokumentation und Darstellung der Ergebnisse für das Unfallgeschehen Allgemein und nach Art der Verkehrsanlage erfolgt in Datenblättern mit folgendem Inhalt:

- Metainformationen

(Informationen über die Region, Betrachtungszeitraum, die Anzahl und Art der auswertbaren Elemente, das Merkmal und die Merkmalsausprägung sowie den evtl. eingesetzten Datenfilter)

- Diagramm

- Kurzbeschreibung und Kurzinterpretation des Diagramms

- Verknüpfungen (Hinweis auf weitere Diagramme ähnlicher Art)

Die Dokumentation und Darstellung der Ergebnisse nach Art der Verkehrsteilnahme erfolgt in Datenblättern mit folgendem Inhalt:

- Diagramm

- Datentabelle

- Kurzinterpretation des Diagramms und der Datentabelle

Die Diagramme zeigen in erster Linie, welche Parameter bei der Verteilung der Unfallmerkmale in höherem oder niedrigerem Maße bei Unfällen mit Straßenbahnen vorzufinden sind. Sie geben damit Hinweise darauf, wo bei Planung und Betrieb von Straßenbahnverkehrsanlagen besondere Sorgfaltspflicht geboten ist.

Die Datenblätter befinden sich vollständig in einem gesonderten Anlagenband (Anlagenband Teil 1, Datenblätter).

Die im Folgenden dargestellten Diagramme und Erläuterungen enthalten die wesentlichsten Erkenntnisse, die im Rahmen der makroskopischen Untersuchung gewonnen werden konnten. 


\subsubsection{Ergebnisse zum Unfallgeschehen Allgemein und nach Art der Verkehrsanlage}

Es sollen folgend Merkmalsausprägungen aller vorliegenden Unfälle aufgezeigt werden. D.h. in den nachstehenden Diagrammen werden zunächst sowohl Knotenpunktunfälle als auch Streckenunfälle gemeinsam berücksichtigt. Aufgrund der jeweiligen Besonderheiten zwischen Unfällen an Knotenpunkten und der freien Strecke sollen differenziert nach diesen Verkehrsanlagen in diesem Kapitel die wesentlichsten Erkenntnisse darüber hinaus beschrieben werden. Der Schwerpunkt liegt dabei auf den Knotenpunktunfällen, da für die Streckenunfälle bereits bei der Netzanalyse (vgl. Kapitel 5) weitreichende Erkenntnisse gewonnen werden konnten, die bei der Auswertung der makroskopischen Analyse bestätigt wurden.

Für die lokalisierten Unfälle wurde der Abstand zu den angrenzenden Knotenpunkten bestimmt. Die Summenkurve der Knotenpunktabstände (Abbildung 6-22) verdeutlicht, dass sich die überwiegende Anzahl der Unfälle mit Personenschaden unter der Beteiligung von Straßenbahnen an Knotenpunkten oder in deren Einflussbereichen ereignen. Bei einem etwas weiter gefassten Knotenpunkteinflussbereich von $50 \mathrm{~m}$ sind $86 \%$ der Unfälle vorzufinden. Innerhalb eines enger gefassten Einflussbereichs von 25 m ereignen sich immer noch $75 \%$ der Unfälle.

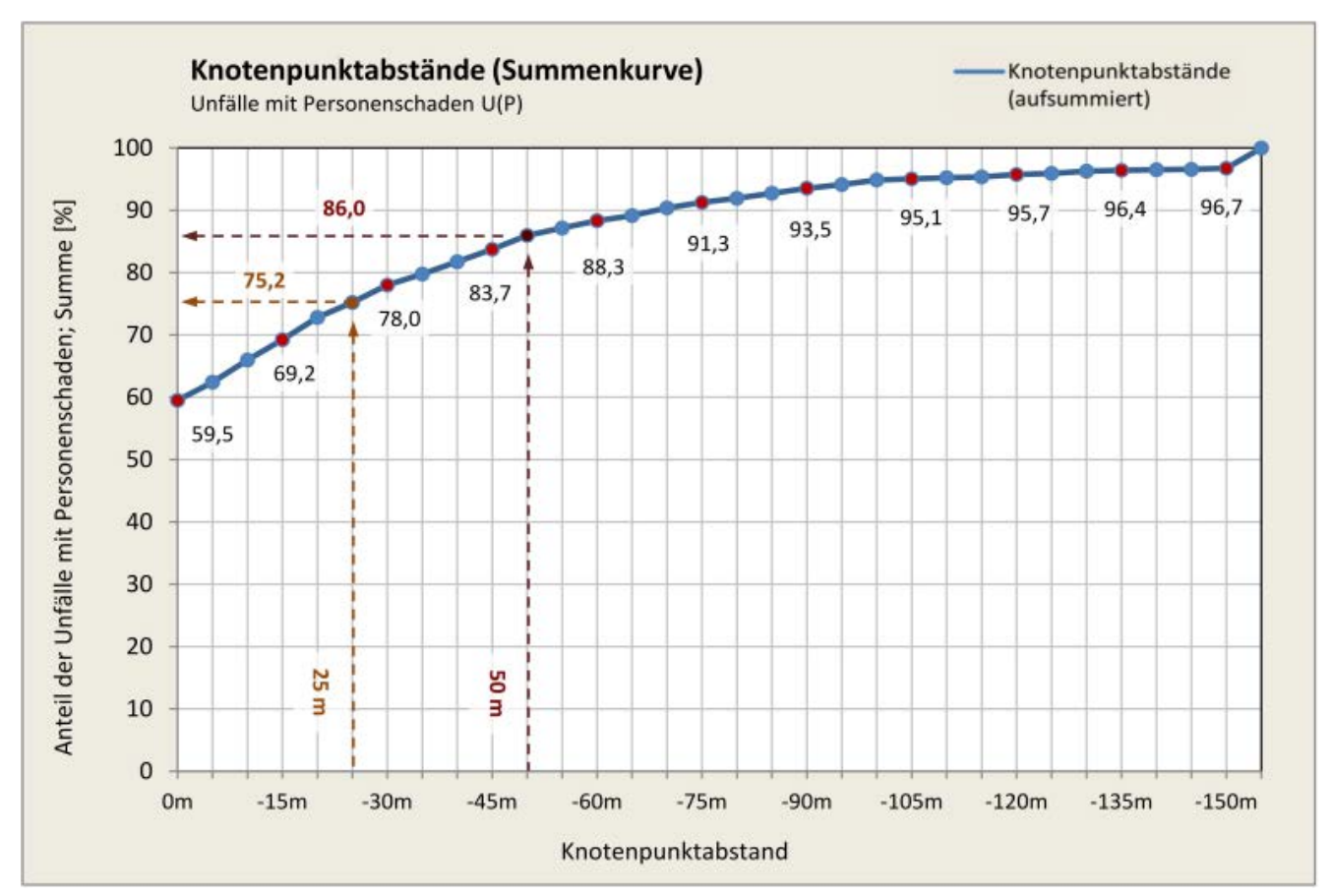

Abbildung 6-22: Knotenpunktabstände (Summenkurve)

Dementsprechend gelten für Knotenpunkten mit Straßenbahnverkehr gleichermaßen, wie für alle Verkehrsanlagen, die Grundsätze einer sicheren Verkehrsanlage in Form von Erkennbarkeit, Begreifbarkeit, Einheitlichkeit, Begeh- bzw. Befahrbarkeit und Übersichtlichkeit.

Für die Auswertungen der makroskopischen Analyse wurden Unfälle als Knotenpunktunfälle definiert, wenn diese 50 m oder weniger von einem Knotenpunkt entfernt waren. Streckenunfälle waren Unfälle mit einem Knotenpunktabstand größer als $50 \mathrm{~m}$.

Ziel der makroskopischen Analyse ist, Unfallhergänge zu verstehen und Beteiligte und Hauptverursacher zu identifizieren.

Aus Abbildung 6-23 ist zu erkennen, dass Straßenbahnen in den wenigsten Fällen selbst Hauptverursacher von Straßenbahnunfällen sind. Nur zu 15,5\% sind Straßenbahnen Beteiligter 01 
(und damit Hauptverursacher) von Straßenbahnunfällen. Von diesen 15,5\% ist ca. 1/3 auf Unfälle zurückzuführen, die sich ohne Beteiligung weiterer Verkehrsteilnehmer ereignet haben. Im Wesentlichen sind das Unfälle, bei dem Straßenbahninsassen gestürzt sind.

Vor allem bei Radfahrern, PKW und motorisierten Zweirädern wird aus Abbildung 6-23 ersichtlich, dass diese im Falle einer Beteiligung auch zu über 85\% Hauptverursacher des Unfalls sind.

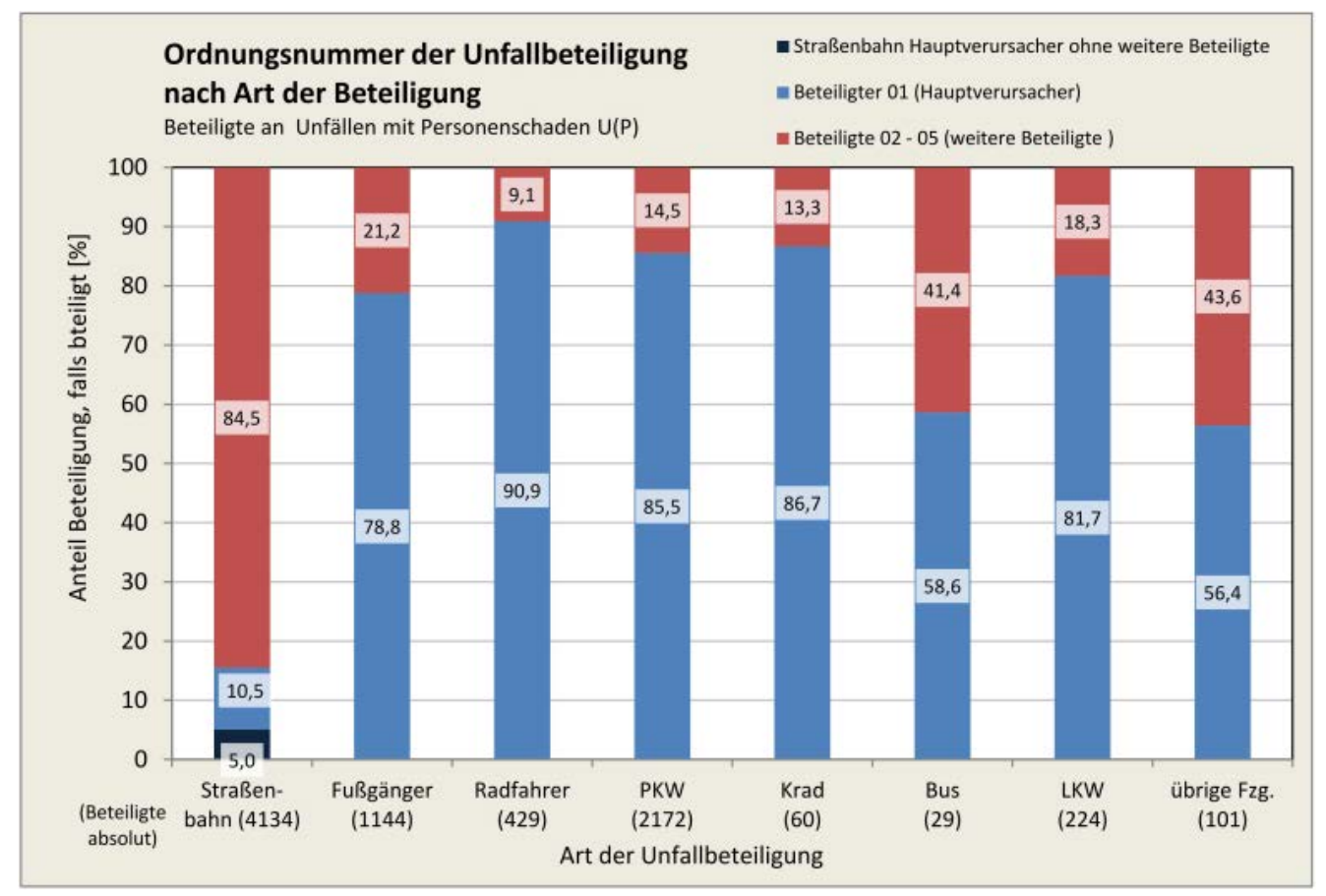

Abbildung 6-23: Ordnungsnummer der Unfallbeteiligung nach Art der Beteiligung

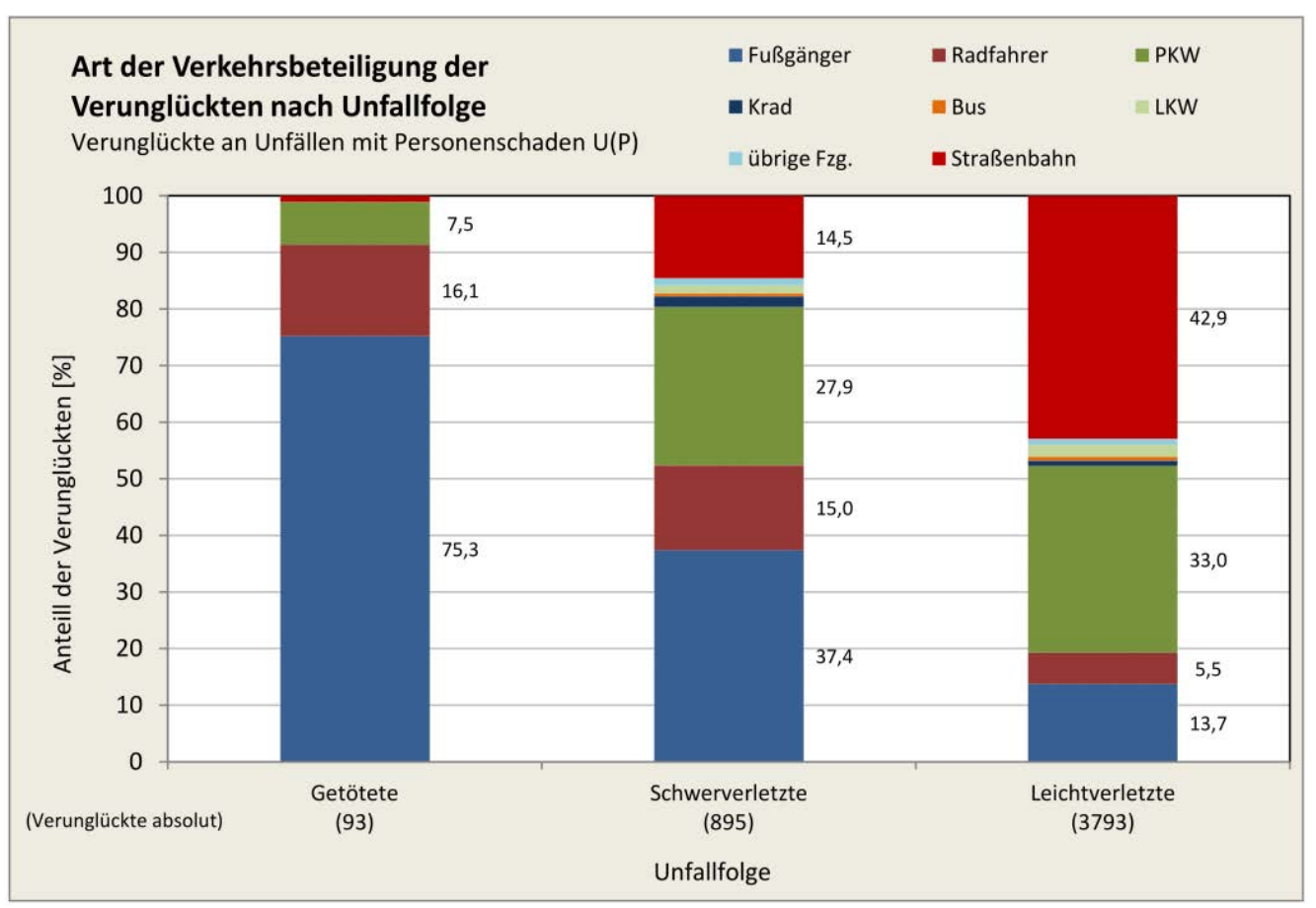

Abbildung 6-24: Art der Verkehrsbeteiligung der Verunglückten nach Unfallfolge

Fußgänger haben bei den Getöteten und bei den Schwerverletzten den weitaus größten Anteil (vgl. Abbildung 6-24). Radfahrer sind bei Unfällen mit schwerer Unfallfolge ebenfalls 
überproportional betroffen. Bei den Leichtverletzten dominieren dagegen die Insassen von Straßenbahnen und PKW. Bei besonderer Betrachtung der Getöteten und Schwerverletzten nach der Lage der Unfallstelle im Netz (Abbildung 6-25) zeigt sich, dass Fußgänger überproportional an Strecken und Haltestellen schwer verunglücken. Absolut ereignen sich die meisten Unfälle mit Schweren Personenschaden auch bei Fußgängern jedoch an den Knotenpunkten.

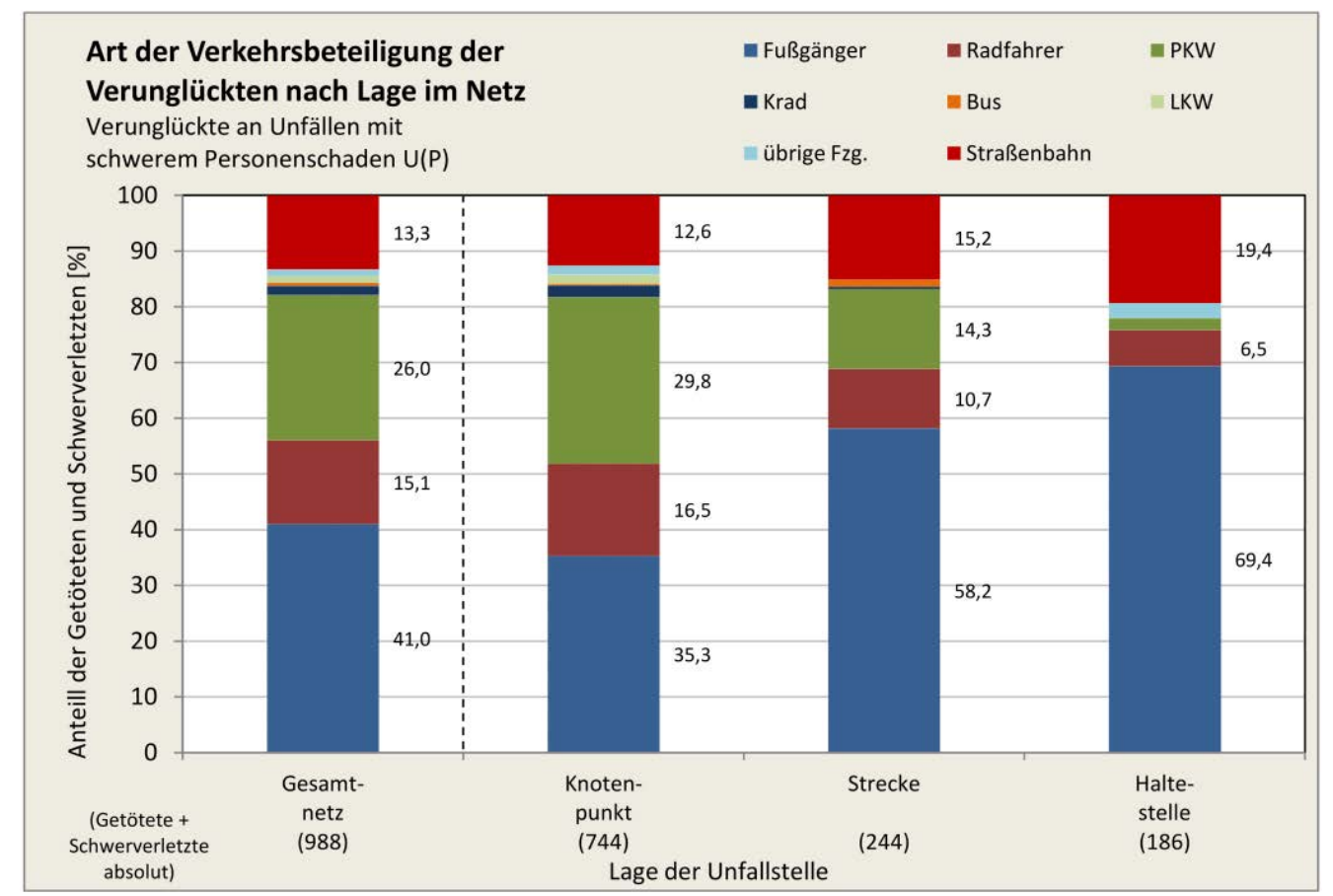

Abbildung 6-25: Art der Verkehrsbeteiligung der Getöteten und Schwerverletzten nach Lage im Netz

Differenziert nach den Hauptverursachern in Abhängigkeit der Unfallkategorie zeigt sich in Abbildung 6-26, dass bei Unfällen mit Getöteten über 75\% auf Fußgänger und Radfahrer zurückzuführen sind. Entsprechend ähnlich sind die Anteile bei den Verletzten.

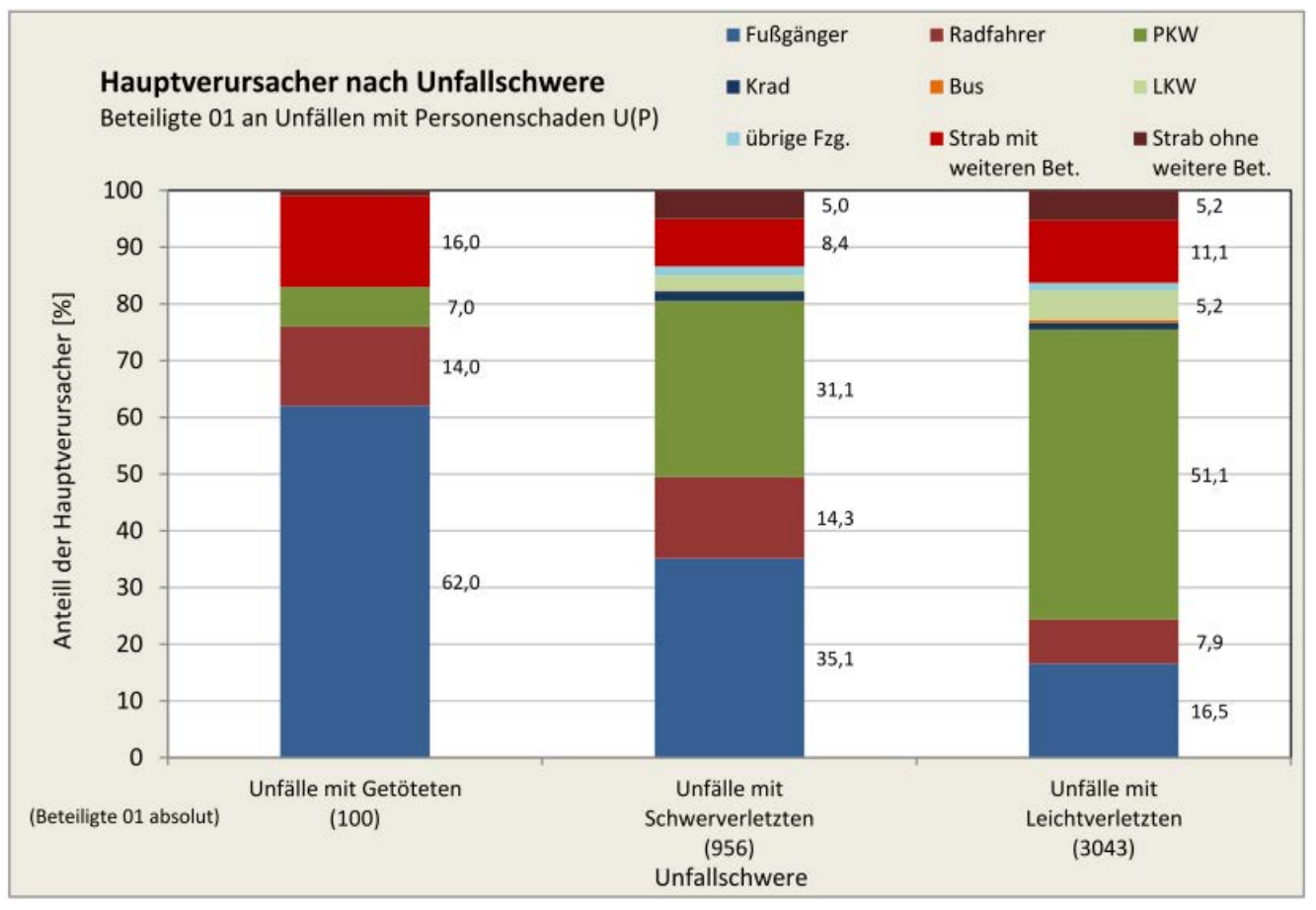

Abbildung 6-26: Hauptverursacher nach Unfallschwere 
Aber vor allem bei den schwersten Unfallfolgen hat die Straßenbahn mit $16 \%$ der Hauptverursacher einen nicht unerheblichen Einfluss auf das Gesamtunfallgeschehen.

Bei den Unfällen mit Schwerverletzten und weitergehend bei den Unfällen mit Leichtverletzten nimmt der Anteil der Fußgänger als Hauptverursacher zu Ungunsten der PKW ab. Außerdem wird deutlich das bei den Unfällen mit Schwer- und Leichtverletzten ca. 1/3 auf Verunglückte in der Straßenbahn ohne Beteiligung weiterer Verkehrsteilnehmer zurückzuführen ist.

Bei einer Differenzierung der Hauptverursacher nach der Lage im Netz, der von ihnen maßgeblich verursachten Unfälle (Abbildung 6-28), ist zu erkennen, dass von den zahlreichen oben beschriebenen Knotenpunktunfällen ein Großteil mit motorisierten Verkehrsteilnehmern als Hauptverursacher und den entsprechenden Verunglückten im Zusammenhang stehen. Die Unfallschwere ist dementsprechend an den Knotenpunkten geringer. Auf der freien Strecke und an Haltestellen dominieren Unfälle mit Fußgängern, wobei an Haltestellen im Vergleich die Geschwindigkeit der Straßenbahn geringer ist. Entsprechend sind an Strecken und Haltestellen die Unfallfolgen gegenüber den Knotenpunkten ungünstiger, wobei die Unfallschwere der Strecke die der Haltstelle noch übersteigt. (s. Anlagenband Teil 1, Datenblätter). Abbildung 6-27 verdeutlicht damit, welchen Verkehrsbeteiligten in Abhängigkeit der Verkehrsanlage besonderer Stellenwert bei der Gestaltung der Verkehrsanlagen einzuräumen ist.

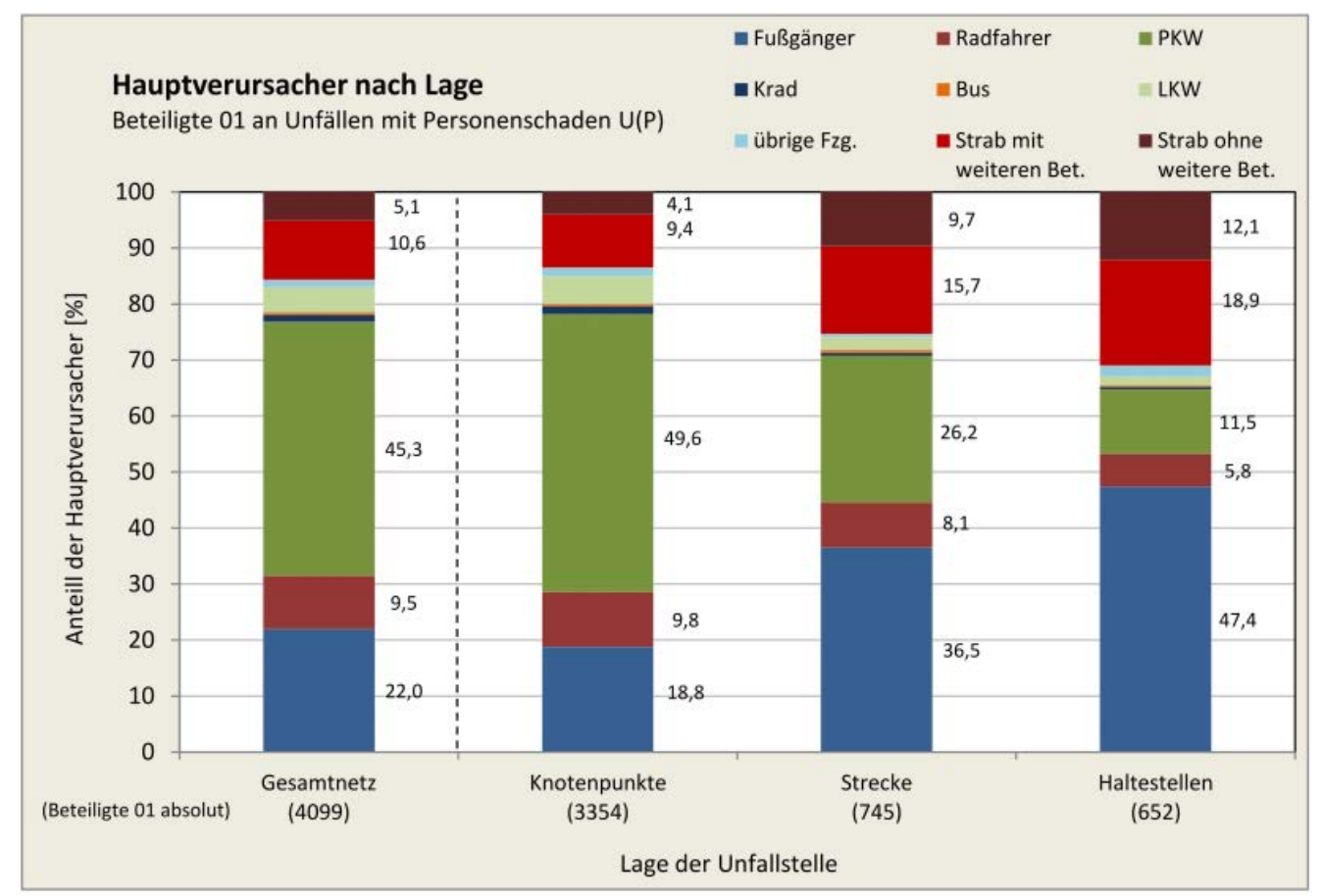

Abbildung 6-27: Hauptverursacher nach Lage

Aus dargelegten Gründen der unterschiedlichen Unfallmerkmale zwischen Knotenpunkten und Strecke ist es erforderlich, die Unfälle differenziert nach der jeweiligen Lage im Netz zu betrachten. 


\section{Unfälle an Knotenpunkten}

Abbildung 6-28 zeigt deutlich, dass sich der überwiegende Anteil der Knotenpunktunfälle an lichtsignalgeregelten Knotenpunkten ereignet. Mit knapp 30\% sind Straßenbahnunfälle an Knotenpunkten mit Vorfahrt-regelnden-Verkehrszeichen ebenfalls häufig. Sicher sind diese hohen Anteile auch darauf zurückzuführen, dass diese Knotenpunktarten vergleichsweise oft als Knotenpunktgrundformen in Zusammenhang mit Straßenbahnanlagen vorgesehen sind. Dennoch ist aufgrund dieser Grafik zu erkennen, dass diesen beiden Knotenpunkttypen bei der verkehrssicheren Gestaltung ein hoher Stellenwert eingeräumt werden muss.

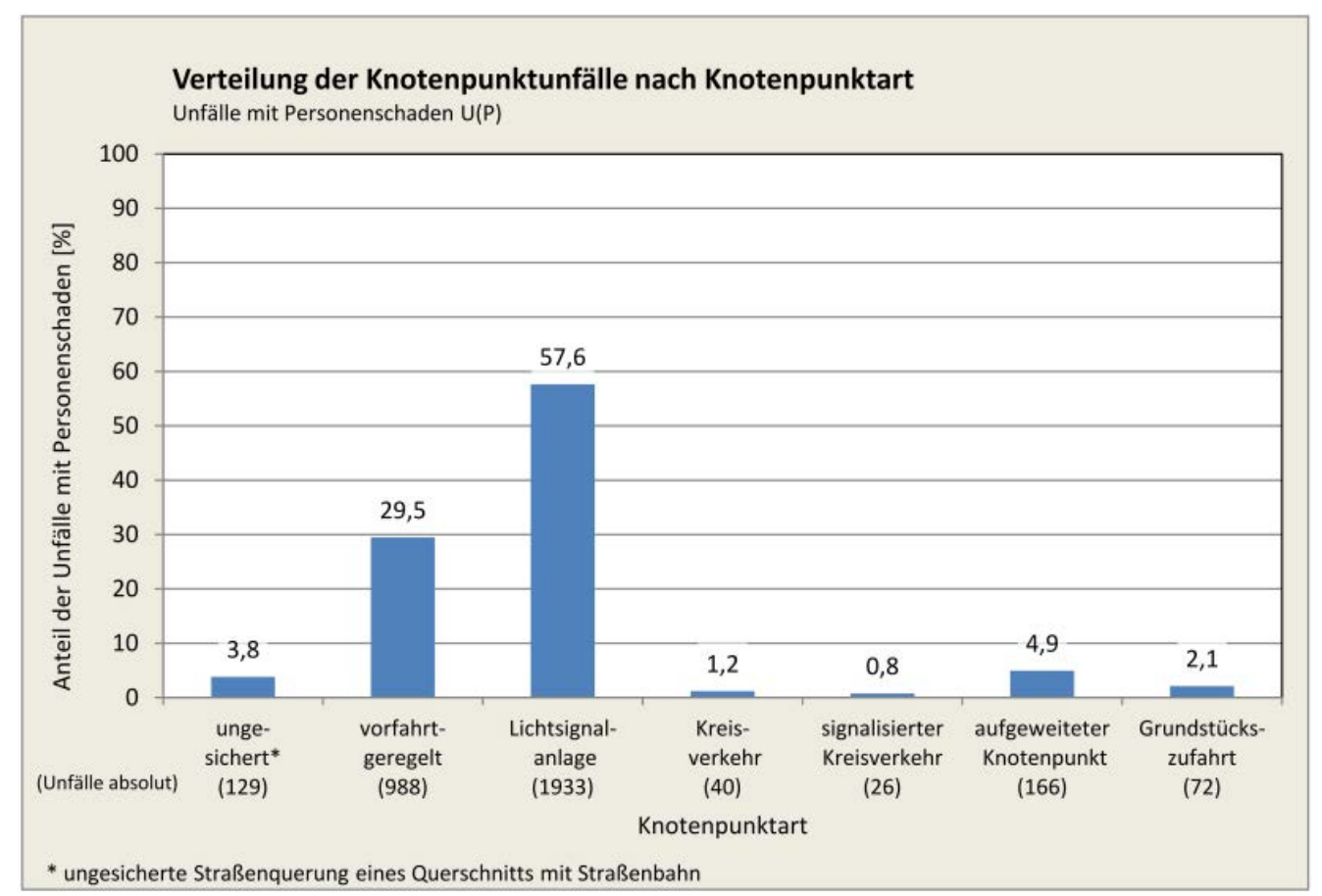

Abbildung 6-28: Anteil der Knotenpunktunfälle nach Knotenpunktart

Aufgrund der hohen Anteile von Unfällen an Lichtsignalknoten und Vorfahrtsknoten wurden diese beiden Knotenpunktarten detaillierten Betrachtungen durch Erstellung gesonderter Diagramme zugeführt (vgl. Anlagenband Teil 1, Datenblätter).

Bei der Betrachtung der Hauptverursacher in Abhängigkeit der Knotenpunktart (vgl. Abbildung 6-29) ist deutlich zu erkennen, dass an Vorfahrtsknoten und Grundstückseinfahrten den Kraftfahrzeugen bei der verkehrssicheren Gestaltung ein hoher Stellenwert einzuräumen ist. Bei Lichtsignalknoten und Kreisverkehrsplätzen ist der Anteil der Fußgänger und Radfahrer als Hauptverursacher (und auch als Verunglückte) überproportional hoch.

Insgesamt zeigte sich, dass Knotenpunkte mit Lichtsignalanlagen nicht nur durch die meisten, sondern (aufgrund der vergleichsweise hohen Beteiligung von Radfahrern und Fußgängern) auch durch die schwersten Unfälle geprägt sind (Abbildung 6-30).

Aufgrund der durchschnittlich höheren Straßenbahnverkehrsstärke an Lichtsignalknoten ist die Unfallkostenrate durch Straßenbahnunfälle dieser Knotenpunktform jedoch niedriger als bei den z.B. schwächer belasteten Kreisverkehrsplätzen. 


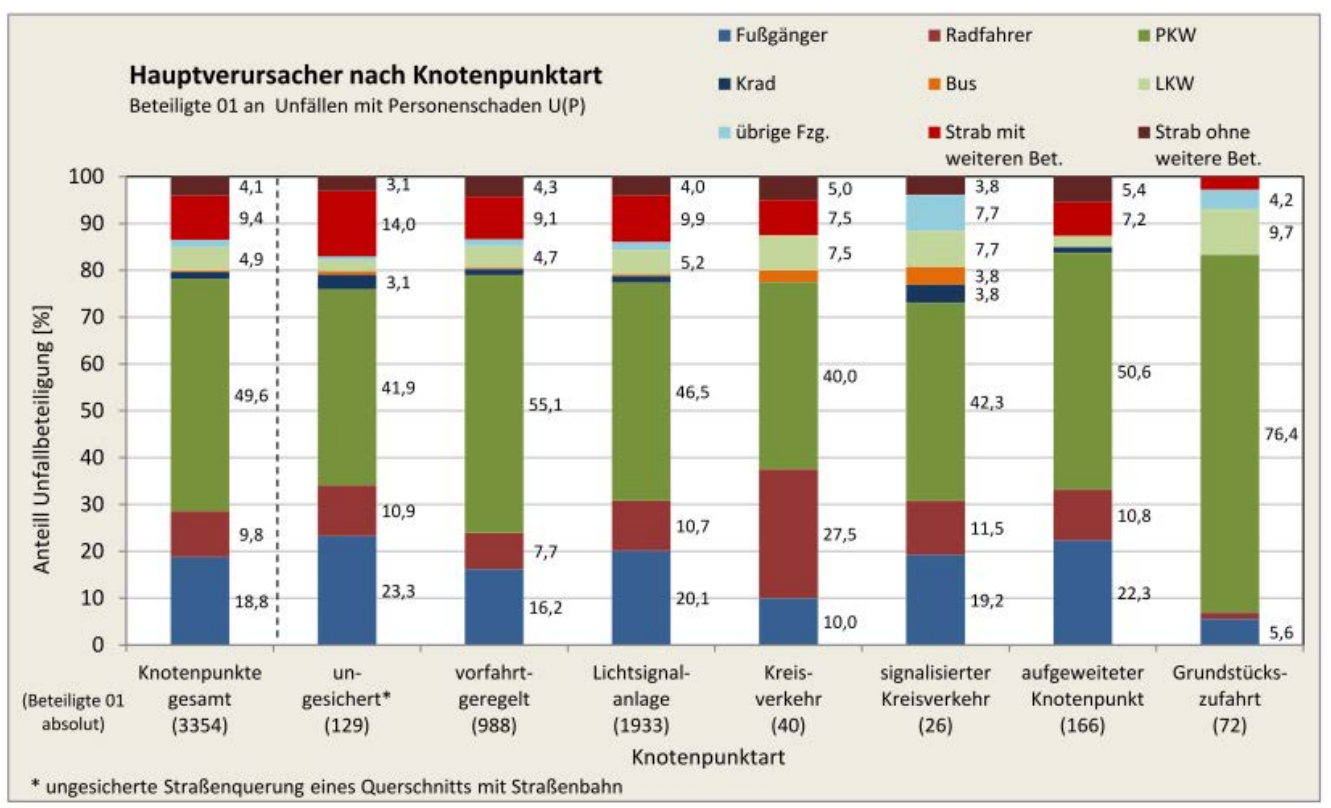

Abbildung 6-29: Hauptverursacher nach Knotenpunktart

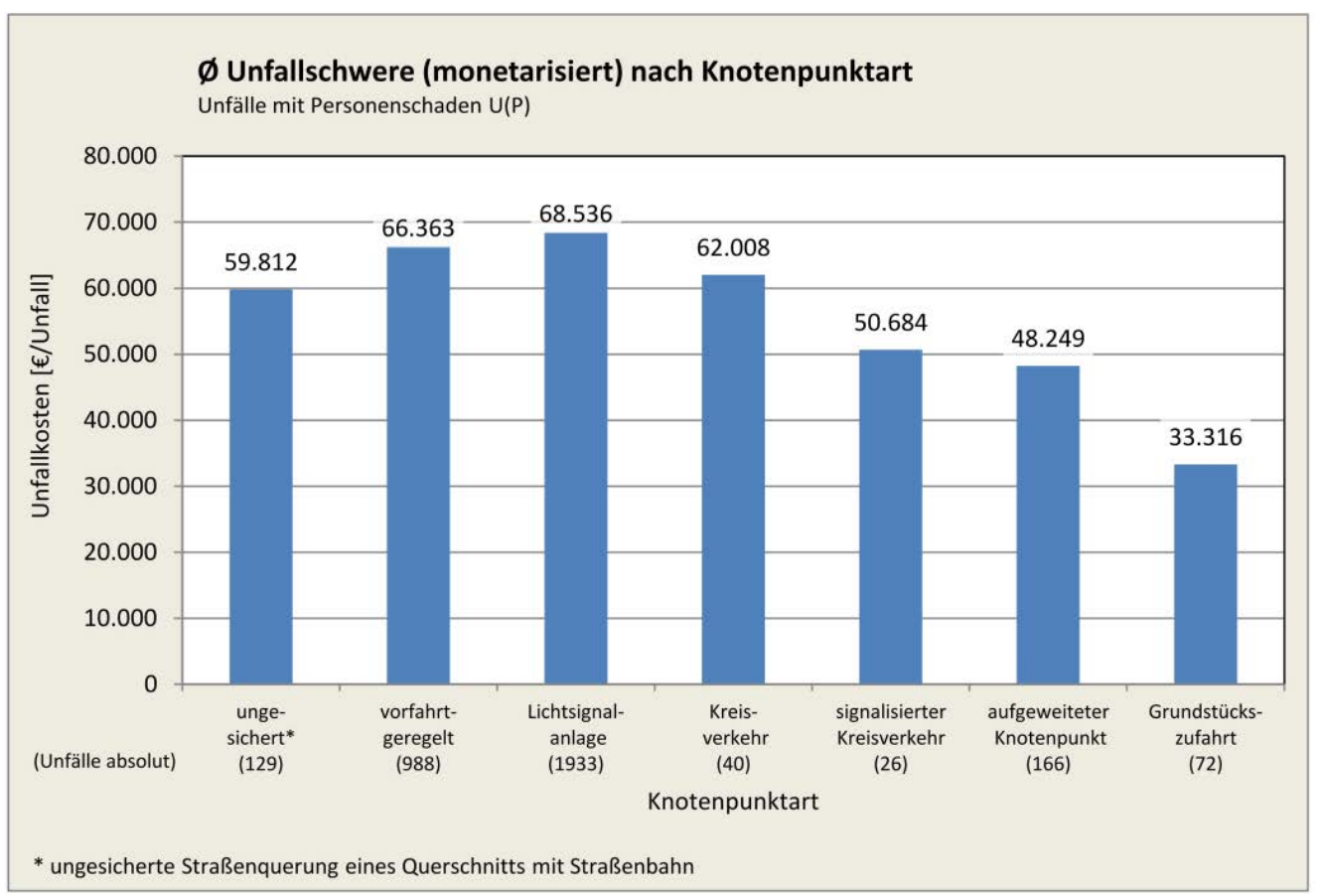

Abbildung 6-30: Unfallschwere nach Knotenpunktart

Aus Abbildung 6-31 sind die Unfallkostenraten nach Knotenpunktart abzulesen. Es muss dabei beachtet werden, dass die Unfallkosten ausschließlich auf Unfälle mit Beteiligung von Straßenbahnen basieren. Die bei der Unfallkostenrate zu Grunde gelegte Verkehrsstärke ist die Straßenbahnverkehrsstärke. Betrachtet wurden für diese Auswertung ausschließlich Knotenpunkte an dem sich ein oder mehrere Unfälle unter Beteiligung von Straßenbahnen ereignet hat. 


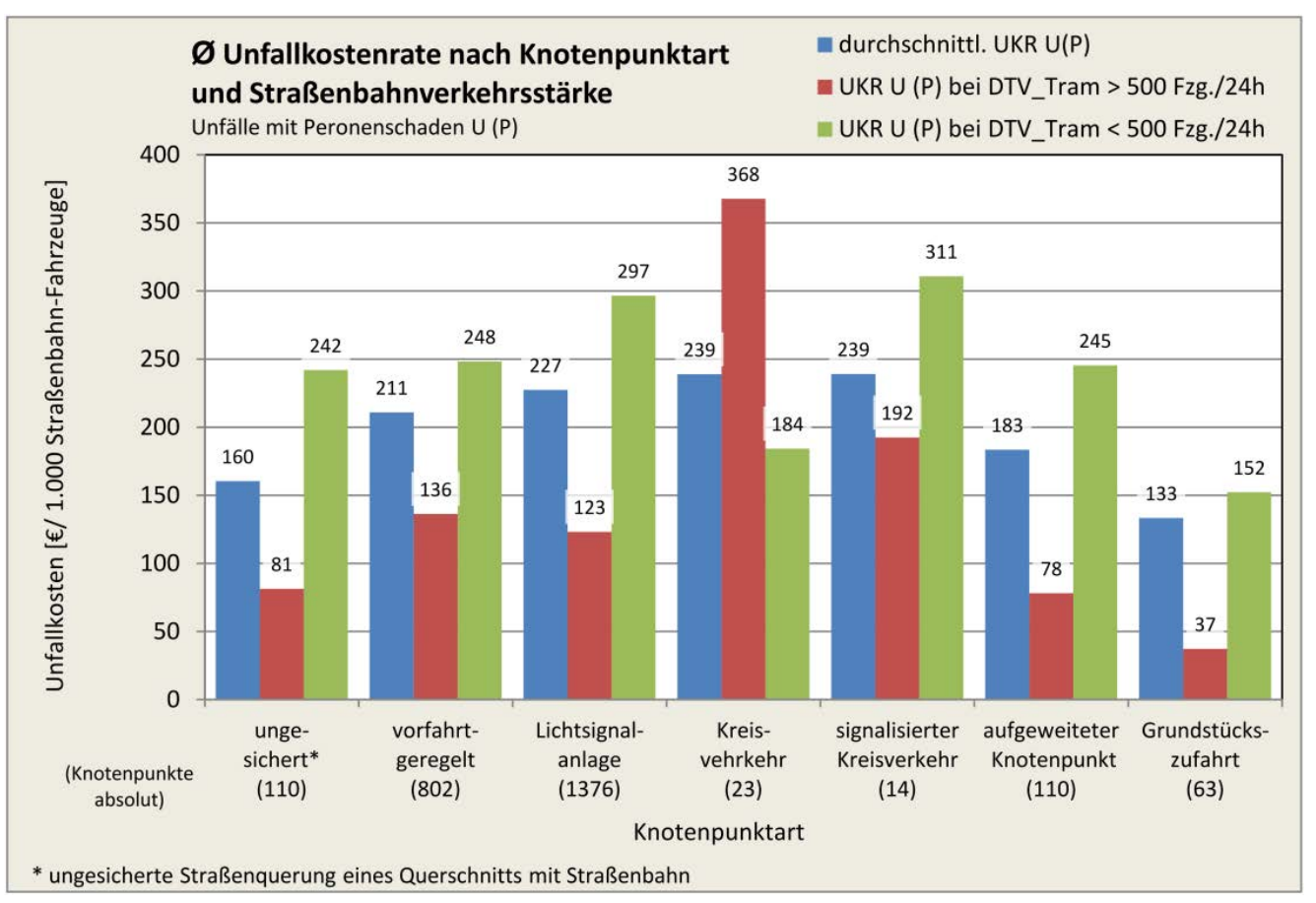

Abbildung 6-31: Unfallkostenrate nach Knotenpunktart und Straßenbahnverkehrsstärke

Für eine bestimmte Knotenpunktart kann an dieser Stelle keine Empfehlung gegeben werden, da für eine abschließende Einschätzung das Unfallgeschehen (analog der Netzanalyse für verschiedene Querschnitte, Kapitel 5) auf die Grundgesamtheit der Knotenpunkte ausgeweitet werden müsste. D.h. es müssten vergleichend auch alle Knotenpunkte ausgewählter Netze berücksichtigt werden, an denen kein Unfall mit Straßenbahnen festzustellen war.

Dennoch können für die hier ausgewerteten Knotenpunkte mit mindestens einem Straßenbahnunfall Rückschlüsse auf die Verkehrssicherheit der verschiedenen Knotenpunktarten gezogen werden: Es ist zu konstatieren, dass lichtsignalgeregelte Knotenpunkte bei höheren Straßenbahnverkehrsstärken eine vergleichsweise sichere Knotenpunktart sind. Vorfahrtsknoten sind nicht immer unsicher. Aufgrund der geringen Fallzahlen ist für Kreisverkehrsplätze eine Aussage kaum möglich. Es konnte jedoch festgestellt werden, dass die Unfallschwere insbesondere an signalisierten Kreisverkehrsplätzen vergleichsweise gering ist. Aufgrund der Unfallanzahl und der vergleichsweise geringen Straßenbahnverkehrsstärken der untersuchten Kreisverkehrsplätze ist die Unfallkostenrate dieser Knotenpunktarten jedoch vergleichsweise hoch.

Bei der Betrachtung der verschiedenen Führungsformen der Straßenbahn in den Knotenpunktzufahrten (Abbildung 6-32) zeigt es sich, dass 3/4 aller von der Straßenbahn befahrenen Querschnitte der Knotenpunkte Bahnkörper in Mittellage waren. 


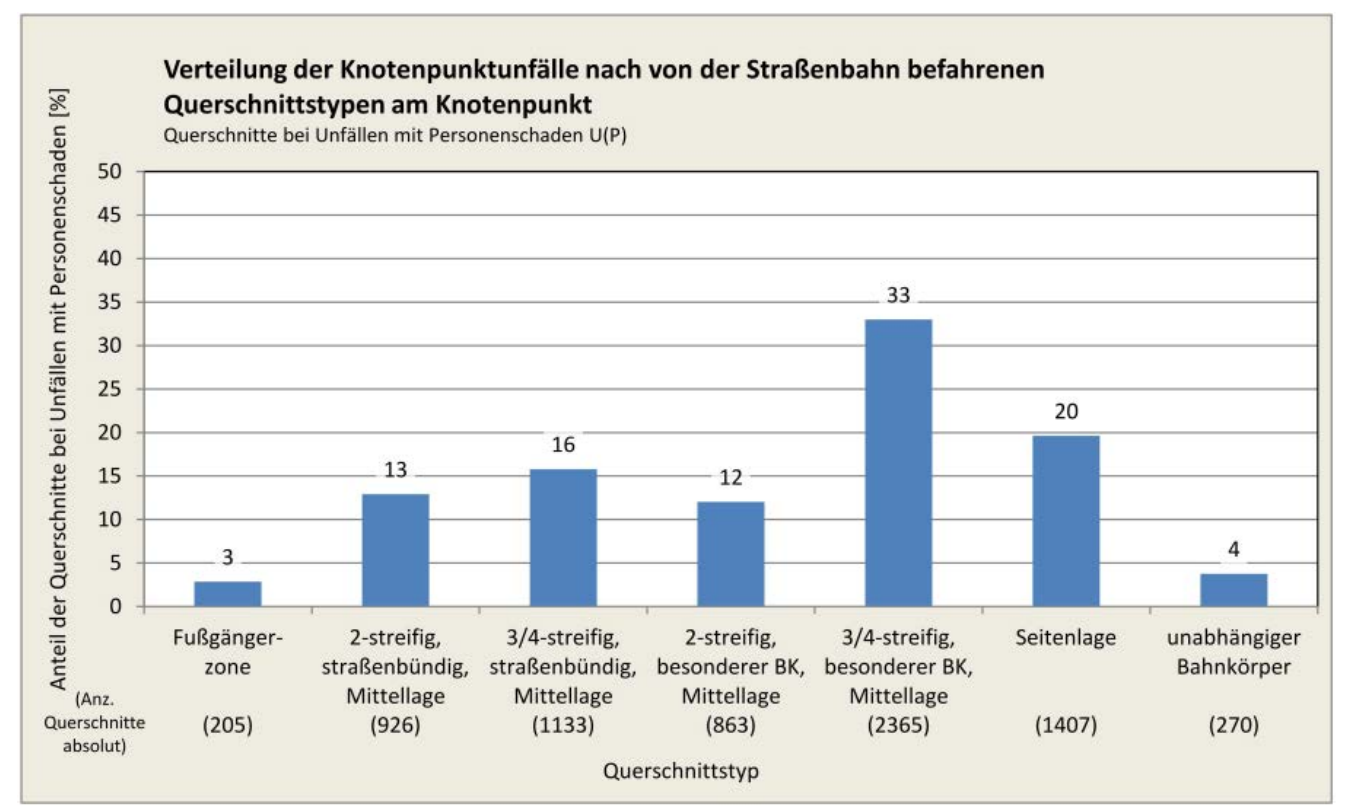

Abbildung 6-32: Knotenpunktunfälle nach der von der Straßenbahn befahrenen Querschnittstypen am Knotenpunkt

Aus Abbildung 6-33 wird deutlich, dass bei Unfällen an Knotenpunkten mit Bahnkörpern in Mittellage überproportional die Kraftfahrzeuge als Hauptverursacher in Erscheinung treten. Eine Ausnahme bilden besondere Bahnkörper in Mittellage mit 3 oder 4 Fahrstreifen des Kfz-Verkehrs. Hier ist der Anteil der Fußgänger und Radfahrer als Hauptverursacher deutlich höher als bei den übrigen Bahnkörpern in Mittellage. Aufgrund des deutlich höheren Anteils der Unfälle nach Querschnittstyp (s. Abbildung 6-32) ist zu schlussfolgern, dass neben den Unfällen mit Kraftfahrzeugen zusätzlich Unfälle mit Fußgängern und Radfahrern bei dieser Führungsform im Knotenpunkt hinzukommen.

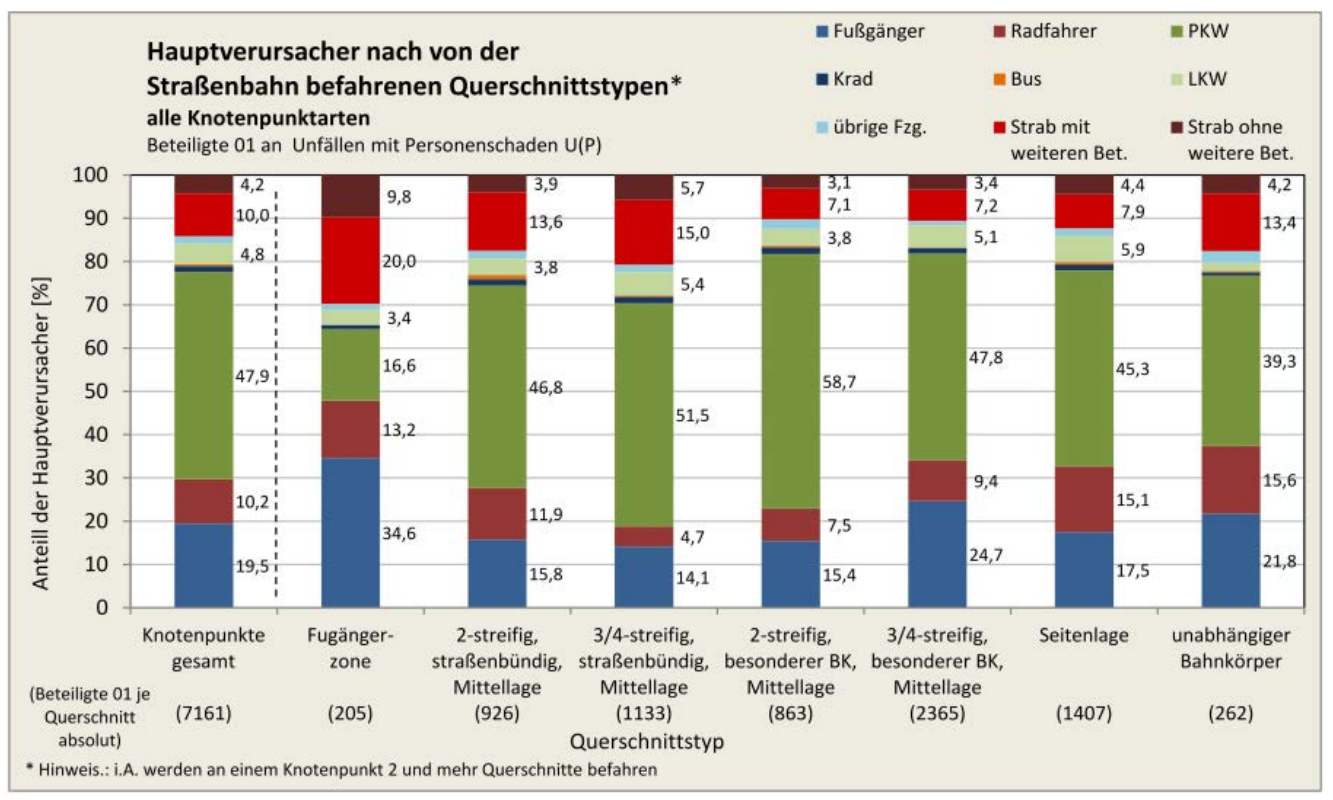

Abbildung 6-33: Hauptverursacher nach von der Straßenbahn befahrenen Querschnittstypen an Knotenpunkten

Das wird bestätigt, in dem die Unfallkostenraten dieses Querschnittstyps aus der Netzanalyse einschließlich der Knotenpunkte nach Abbildung 5-6, S.64 betrachtet wird. Es zeigt sich, dass die Unfallkostenrate dabei ca. doppelt so hoch ist, als bei den übrigen Querschnittstypen. 


\section{Unfälle der freien Strecke}

Wesentliche Erkenntnisse für Straßenbahnunfälle der freien Strecke konnten bereits aus der Netzanalyse (s. Kapitel 5) gewonnen werden.

Vergleichende und weiterführende Diagramme für alle vorliegenden Straßenbahnunfälle wurden (analog zu den Knotenpunkten) auch für die Streckenunfälle erarbeitet.

Als Streckenunfälle gelten Unfälle mit einem Abstand größer 50 m zu einem Knotenpunkt.

Die in Kapitel 5 ermittelten Ergebnisse hinsichtlich Verkehrsteilnehmerarten bei den Hauptverursachern und Verunglückten sowie hinsichtlich Unfalltypen, Unfallarten und Unfallschwere wurden durch die Ausweitung der Auswertung auf alle vorliegenden Straßenbahnunfälle bestätigt. Abbildung 4-1 zeigt beispielhaft die Unfallschwere der Streckenunfälle in Abhängigkeit vom Querschnittstyp. Es wird deutlich, dass besondere Bahnkörper in Mittellage mit 3 oder 4 Fahrstreifen des KfZ-Verkehrs die durchschnittlich höchste Unfallschwere aufweisen, die im Zusammenhang mit der vergleichsweise hohen Anzahl von Unfällen bei diesem Querschnittstyp (wie in Kapitel 5 beschrieben) zu der höchsten Unfallkostenrate beiträgt. Es ist zu erkennen, dass Bahnkörper in Seitenlage und unabhängige Bahnköper ebenfalls Unfälle mit einer vergleichsweise hohe Unfallschwere zur Folge haben. Die in Kapitel 5 beschriebene sehr geringe Unfalldichte führt jedoch dazu, dass die Bahnkörper insgesamt gesehen die sichersten Formen der Führung von Straßenbahnen darstellen.

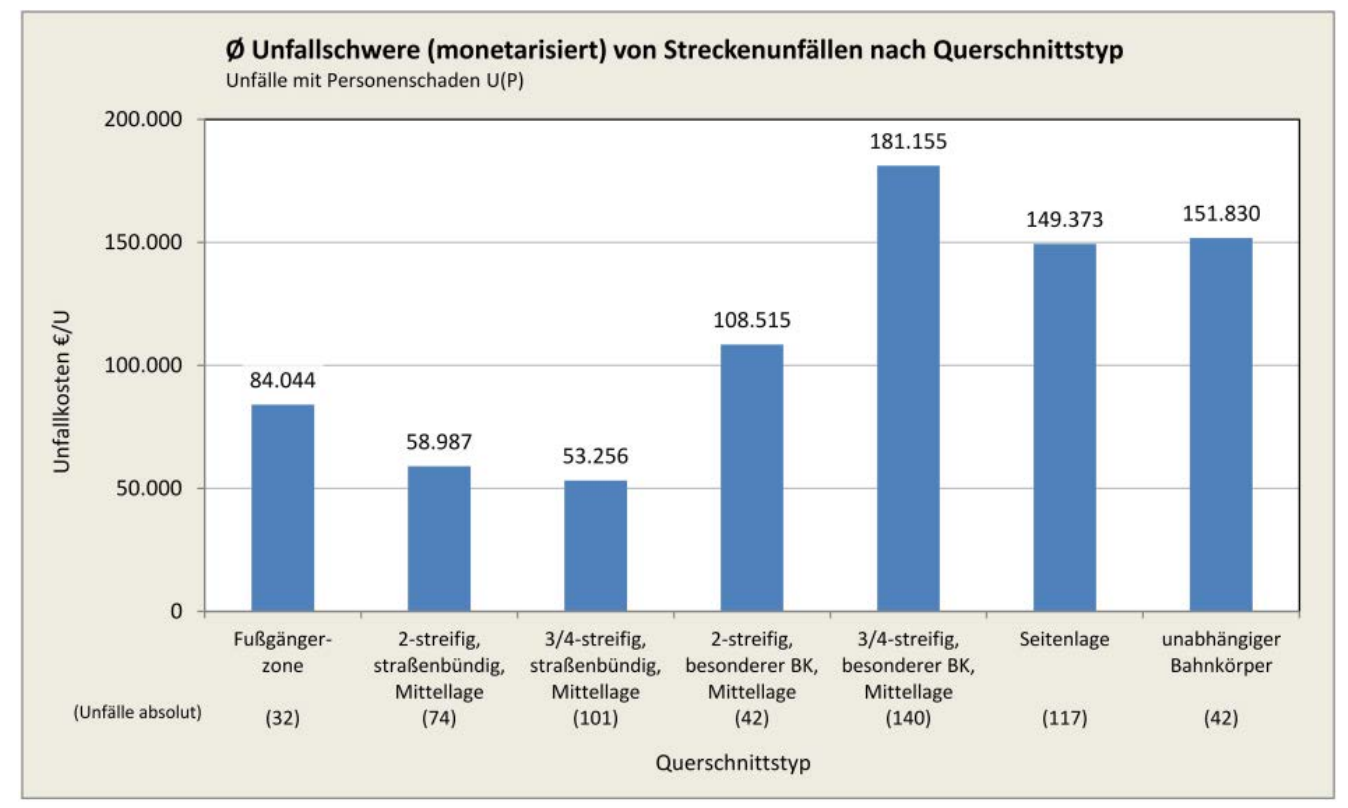

Abbildung 6-34: Unfallschwere von Streckenunfällen nach Querschnittstypen 


\subsubsection{Sicherheitsbewertung nach Art der Verkehrsteilnahme als Hauptverursacher}

Neben den weitreichenden Erkenntnissen der Sicherheitsbewertung nach Art der Verkehrsanlage, werden im Folgenden die Ergebnisse der Analyse nach Art der Verkehrsteilnahme als Hauptverursacher vorgestellt. Basis dafür sind die im Anhangsband aufgeführten Datenblätter. Diese geben nach Art der Verkehrsteilnahme weitere Hinweise darauf, welche unfallbegünstigenden Umstände einen Einfluss auf Unfälle mit Personenschaden unter Beteiligung von Straßenbahnen haben.

Um Auffälligkeiten nach Art der Verkehrsteilnahme als Hauptverursacher herausarbeiten zu können, wurden die Unfälle mit der jeweiligen Beteiligungsart dem Gesamtunfallgeschehen mit Straßenbahnen gegenübergestellt. Das Ergebnis sind Informationen darüber, ob bestimmte Beteiligungsarten über- oder unterproportional häufig nach bestimmten Unfallmerkmalen oder nach bestimmten Verkehrsanlagen am Unfallgeschehen mit Straßenbahnen als Hauptverursacher in Erscheinung treten.

\subsubsection{Fußgänger}

- Fußgänger sind überwiegend bei Überschreiten-Unfällen Hauptverursacher.

- Unfälle mit Fußgängern sind durch eine vergleichsweise hohe Unfallschwere geprägt.

- Fußgänger sind überwiegend und überdurchschnittlich Unfallverursacher bei Unfällen mit Straßenbahnen.

- Überdurchschnittlich jungen Menschen (bis 17 Jahre) und überdurchschnittlich ältere Menschen (ab 75 Jahre) sind als Fußgänger Hauptverursacher bei Unfällen mit Straßenbahnen.

- Absolut betrachtet ereignen sich Unfälle mit Beteiligung von Fußgängern als Hauptverursacher überwiegend an Knotenpunkten trotz grundsätzlichem Vorhandensein von Querungshilfen.

- Im Vergleich zu allen Unfällen mit Straßenbahnen ereignen sich darüber hinaus überproportional viele Unfälle mit Beteiligung von Fußgängern als Hauptverursacher außerhalb der Knotenpunkte und an Haltestellen.

- Unfälle mit Beteiligung von Fußgängern als Hauptverursacher an Knotenpunkten ereignen sich überwiegend und überproportional an Knotenpunkten mit Lichtsignalanlage.

- Es ereignen sich überproportional viele Unfälle mit Fußgängern als Hauptverursacher an Knotenpunkten mit 3- und 4-streifigen besonderen Bahnkörpern in Mittellage und in Fußgängerzonen, in Geschäftsstraßen und Quartiersstraßen sowie bei hohen Straßenbahnverkehrsstärken.

- Es ereignen sich überwiegend und überproportional viele Unfälle mit Fußgängern als Hauptverursacher bei Strecken mit 3- und 4-streifigen besonderen Bahnkörpern in Mittellage bei Strecken in Seitenlage und unabhängigen Bahnkörpern.

- Es ereignen sich überwiegend und überproportional viele Unfälle mit Fußgängern in Verbindungsstraßen sowie überproportional viele Unfälle in Geschäftsstraßen und bei hohen Straßenbahnverkehrsstärken.

- Unfälle mit Beteiligung von Fußgängern als Hauptverursacher an Haltestellen ereignen sich überwiegend und überproportional an Haltestellen mit der Form Mittellage, Wartefläche und Haltefläche zusammenliegend. 


\subsubsection{Radfahrer}

- Unfälle mit Radfahrern als Hauptverursacher sind überwiegend und überproportional bei Einbiegen-/ Kreuzen-Unfällen zu erkennen.

- Unfälle mit Radfahrern als Hauptverursacher sind durch eine vergleichsweise hohe Unfallschwere geprägt.

- Bei Unfällen mit Radfahrern sind diese zu 90,9\% für den Unfall Hauptverursacher und damit weit überdurchschnittlich oft für den Unfall verantwortlich.

- Überdurchschnittlich viele Kinder und junge Erwachsene sind als Radfahrer Hauptverursacher von Unfällen mit Straßenbahnen.

- Die meisten Unfälle mit Straßenbahnen und Radfahrern als Hauptverursacher ereignen sich an Knotenpunkten.

- Überdurchschnittlich viele Radfahrer als Hauptverursacher verunglücken an Querungsstellen der Knotenpunkte oder der freien Strecke.

- Unfälle mit Beteiligung von Radfahrern als Hauptverursacher ereignen sich überwiegend und überproportional an Knotenpunkten mit Lichtsignalanlage und überproportional häufig an Kreisverkehren.

- Vergleichsweise häufig treten Unfälle mit Radfahrern als Hauptverursacher bei Knotenpunkten mit den Gleisen in Seitenlage, bei Knotenpunkten mit anbaufreien Zufahrten und bei Knotenpunkten mit hohen Straßenbahnverkehrsstärken auf.

- Es ereignen sich überwiegend und überproportional viele Unfälle mit Radfahrern als Hauptverursacher bei Strecken mit 3- und 4-streifigen besonderen Bahnkörper in Mittellage bei Strecken in Seitenlage und unabhängigen Bahnkörpern.

- Vergleichsweise häufig treten Unfälle mit Radfahrer als Hauptverursacher bei anbaufreien Strecken und bei Strecken mit hohen Straßenbahnverkehrsstärken auf.

- Bei Unfällen an Haltestellen sind am häufigsten und überproportional Haltestellen mit Seitenlage der Gleise betroffen.

\subsubsection{Kraftfahrzeuge}

- Fahrzeuge des motorisierten Verkehrs als Hauptverursacher sind überwiegend und überproportional in Abbiegen und Einbiegen-/ Kreuzen-Unfälle mit Straßenbahnen involviert.

- Unfälle mit Fahrzeugen des motorisierten Verkehrs als Hauptverursacher und Straßenbahnen sind durchschnittlich weniger schwer als bei anderen Verkehrsteilnehmern.

- Beteiligte des motorisierten Verkehrs sind überwiegend und überdurchschnittlich Hauptverursacher von Unfällen mit Straßenbahnen.

- Unfälle mit Fahrzeugen des motorisierten Verkehrs als Hauptverursacher ereignen sich überwiegend und überdurchschnittlich an Knotenpunkten.

- Haltestellen des Straßenbahnverkehrs und Straßenbahnquerungsstellen für Fußgänger und Radfahrer spielen dabei eine vergleichsweise untergeordnete Rolle.

- Die meisten Unfälle mit Fahrzeugen des motorisierten Verkehrs als Hauptverursacher ereignen sich an lichtsignalgeregelten Knotenpunkten.

- Darüber hinaus sind Fahrzeuge des motorisierten Verkehrs als Hauptverursacher vergleichsweise häufig bei Unfällen an Knotenpunkten mit Vorfahrt-regelnden-Verkehrszeichen und Grundstückszufahrten beteiligt. 
- Überdurchschnittlich häufig sind Fahrzeuge des motorisierten Verkehrs als Hauptverursacher bei Knotenpunkten auf 2-streifigen Straßen mit besonderen Bahnkörpern in Mittellage, 3- bzw. 4-streifigen straßenbündigen Bahnkörpern in Mittellage und vergleichsweise geringen Straßenbahnverkehrsstärken involviert.

- Überdurchschnittlich häufig sind Fahrzeuge des motorisierten Verkehrs als Hauptverursacher bei Strecken mit straßenbündigen Bahnkörper und Strecken mit vergleichsweise geringen Straßenbahnverkehrsstärken involviert.

- Vergleichsweise häufig ereignen sich Unfälle mit Fahrzeugen des motorisierten Verkehrs als Hauptverursacher an Haltestellen in Mittellage.

\subsubsection{Straßenbahnen}

- Bei Unfällen mit Personenschaden und als Hauptverursacher Straßenbahn entfällt der größte Anteil der Verunglückten auf Straßenbahn-Passagiere $(36,1 \%)$, die in der Straßenbahn verunglückt sind.

- Mehr als 1/3 aller der Unfälle mit Personenschaden und als Hauptverursacher Straßenbahn sind ohne weitere Verunglückte Verkehrsteilnehmer außerhalb der Straßenbahn.

- Über die Hälfte der Unfälle mit Straßenbahn als Hauptverursacher wurden polizeilich nach Unfalltyp als "sonstige Unfälle" aufgenommen. Es ist zu mutmaßen, dass es sich dabei überwiegend um Unfälle handelt, bei der Passagiere in der Straßenbahn gestürzt sind.

- Unfälle mit Straßenbahn als Hauptverursacher ereignen sich überproportional häufig außerhalb der Knotenpunkte an Haltestellen und an Querungsstellen der freien Strecke.

- Unfälle mit Hauptverursacher Straßenbahn an Knotenpunkten ereignen sich vergleichsweise häufig an lichtsignalgeregelten und ungesicherten Knoten, in Fußgängerzonen und an Knoten mit straßenbündigen Bahnkörpern.

- Überproportional geschehen Unfälle mit Hauptverursacher Straßenbahn an Knoten von Geschäftsstraßen.

- Unfälle mit Hauptverursacher Straßenbahn auf Strecken ereignen sich vergleichsweise häufiger in Fußgängerzonen, auf straßenbündigen Bahnkörpern und Bahnkörpern in Seitenlage.

- Überproportional geschehen Unfälle mit Hauptverursacher Straßenbahn auf anbaufreien Strecken und bei vergleichsweise geringen Straßenbahnverkehrsstärken.

- Überproportional oft ereignen sich Unfälle mit Hauptverursacher Straßenbahn an Haltestellen in Seitenlage. 


\subsection{Zusammenfassung der makroskopischen Analyse}

Diagramme der Häufigkeitsverteilungen verschiedener Unfallmerkmale (Unfalltypen, Unfallarten, strukturelle Gemeinsamkeiten) geben Hinweise darauf, an welcher Stelle bei Planung und Betrieb von Straßenbahnverkehrsanlagen in Abhängigkeit von Verkehrsteilnehmerart und Form der Verkehrsanlage besondere Sorgfaltspflicht geboten ist.

Damit ist es möglich, auch bei z.B. vergleichsweise sicheren Verkehrsanlagen, Auffälligkeiten zu identifizieren, dann wenn Unfallmerkmale oder Verkehrsbeteiligungen überproportional häufig zu erkennen sind.

Insbesondere 3- bzw. 4-streifige Bahnkörper und lichtsignalgeregelte Knotenpunkte weisen eine hohe Unfallschwere mit hohem Anteil Überschreiten-Unfällen auf.

Bahnkörper in Seitenlage und unabhängige Bahnkörper sind die sichersten Querschnittsformen, allerdings ist bei diesen Querschnitten ein besonderes Augenmerk auf Straßenbahnen und Fußgänger als Hauptverursacher zu richten. 
Um Rückschlüsse auf mögliche unfallbegünstigende Umstände der einzelnen Verkehrsanlagen zu erhalten, sollten durch Vor-Ort-Begehungen für einzelne besonders unfallauffällige Knotenpunkte und Strecken eine Detailanalyse durchgeführt werden.

Ziel war es, weitere straßenräumliche Merkmale und Besonderheiten aufzunehmen sowie Defizite der Infrastruktur zu erkennen.

\subsection{Kriterien für die Auswahl der zu untersuchenden Verkehrsanlagen}

\section{Unfallstellen der freien Strecke}

Bei der Kartierung und der anschließenden Bewertung möglicher zu untersuchender Streckenabschnitte zeigte sich, dass es sich bei Unfällen der freien Strecke weitestgehend um Einzelunfälle handelt. Zwei oder mehr Unfälle auf vergleichsweise kurzer Distanz ohne Änderung der Querschnittsmerkmale waren kaum vorzufinden. Das lässt erkennen, dass Unfallkonzentrationen i.A. an Knotenpunkten oder ggf. auch an höher frequentierten Grundstückszufahrten vorzufinden sind.

Lediglich 11 Streckenabschnitte wurden identifiziert, die auf einer Distanz kleiner $500 \mathrm{~m}$ mehr als einen Unfall außerhalb von Knotenpunkteinflüssen aufwiesen.

Aufgrund der geringen Anzahl der erkannten Abschnitte erübrigte sich eine weitere Eingrenzung der Streckenabschnitte, die für die Ortsbesichtigung in Frage kommen. Es wurden alle 11 Streckenabschnitte im Rahmen der mikroskopischen Analyse betrachtet:

Tabelle 7-27: Streckenauswahl der mikroskopischen Analyse

\begin{tabular}{|c|l|l|c|}
\hline \multicolumn{1}{|c|}{ Stadt } & \multicolumn{1}{c|}{ Unfallstelle } & \multicolumn{1}{c|}{$\begin{array}{c}\text { Länge des } \\
\text { Untersuchungsabschnitts }\end{array}$} \\
\hline 1 & Köln & $\begin{array}{l}\text { Amsterdamer Straße } \\
\text { von Innere Kanalstraße bis Johannes-Müller-Straße }\end{array}$ & $300 \mathrm{~m}$ \\
\hline 2 & Karlsruhe & $\begin{array}{l}\text { Unabhängiger Bahnkörper } \\
\text { von Karl-Wolf-Weg bis Siegfried-Kühn-Straße }\end{array}$ & $450 \mathrm{~m}$ \\
\hline 3 & Düsseldorf & $\begin{array}{l}\text { Oberkasseler Brücke/ Hofgartenrampe } \\
\text { von Luegplatz bis Maximilian-Weyhe-Allee. }\end{array}$ & $400 \mathrm{~m}$ \\
\hline 4 & Halle & $\begin{array}{l}\text { An der Magistrale } \\
\text { von Zur Saaleaue bis Gimritzer Damm (Rennbahnkreuz) }\end{array}$ & $4.200 \mathrm{~m}$ \\
\hline 5 & $\begin{array}{l}\text { Frankfurt } \\
\text { (Main) }\end{array}$ & $\begin{array}{l}\text { Hanauer Landstr. } \\
\text { von Intzestaße bis Schwedlerstraße }\end{array}$ & $400 \mathrm{~m}$ \\
\hline 8 & Bonn & $\begin{array}{l}\text { Am Hauptbahnhof } \\
\text { von Busbahnhof bis Thomas-Mann-Straße }\end{array}$ & $400 \mathrm{~m}$ \\
\hline 7 & Stuttgart & $\begin{array}{l}\text { Probststraße } \\
\text { von Rembrandtstraße bis Balinger Straße }\end{array}$ & $\begin{array}{l}\text { Stuttgart } \\
\text { Engelbergstraße }\end{array}$ \\
\hline
\end{tabular}




\begin{tabular}{|c|c|c|c|}
\hline & Stadt & Unfallstelle & $\begin{array}{l}\text { Länge des } \\
\text { Untersuchungsabschnitts }\end{array}$ \\
\hline 9 & Cottbus & $\begin{array}{l}\text { Gerhart-Hauptmann-Straße } \\
\text { von Nordring bis Am Großen Spreewehr }\end{array}$ & $640 \mathrm{~m}$ \\
\hline 10 & Erfurt & $\begin{array}{l}\text { Warschauer Straße, Vilniuser Straße } \\
\text { von Berliner Straße bis Mainzer Straße }\end{array}$ & $650 \mathrm{~m}$ \\
\hline 11 & Heidelberg & $\begin{array}{l}\text { Eppelheimer Straße } \\
\text { von Henkel-Teroson-Straße bis Czernyring }\end{array}$ & $250 \mathrm{~m}$ \\
\hline
\end{tabular}

\section{Unfallstellen an Knotenpunkten}

Bei der Auswahl der Knotenpunkte war es notwendig eine Auswahl zu treffen. Es sollten zunächst 20 unfallauffällige Knotenpunkte gewählt werden, die für eine genauere Bewertung in Form einer Ortsbegehung geeignet schienen. Als unfallauffällig galten Unfallstellen, die aufgrund der Anzahl, Schwere, Beteiligung oder Struktur der Unfälle gegenüber anderen Unfallstellen ausgeprägt sind. Darüber hinaus sollten mindestens je zwei Knotenpunkte der verschiedenen Knotenpunktformen begutachtet werden.

Als Vorbereitung für eine Auswahl der Knotenpunkte war es erforderlich, die Einzelunfälle aus der Excel-Unfalldatengesamtliste (vgl. Kapitel 6.1.2) einer Unfallstelle exakt zuzuordnen. Das bedeutet, das Unfälle an ein und demselben Knotenpunkt als solches erkannt werden mussten.

Um die Unfälle der einzelnen Knotenpunkte zu ermitteln, wurde ein VBA-Code ${ }^{107}$ in die Gesamtunfalltabelle implementiert. Über die Knotenmerkmale und die Georeferenzierung (geographische Koordinaten) wurde einem Knotenpunkt mit mehr als einem Unfall eine eindeutige Unfallstellenkennung gegeben. Unfälle mit gleicher Knotenpunktkennung ereigneten sich folglich auch an gleichem Knotenpunkt. Die Unfälle der einzelnen Unfallstellen mit allen relevanten Merkmalen konnten daraufhin ausgezählt werden. Darüber hinaus war es möglich die Unfallkenngrößen (Unfallkosten, Unfallkostenraten) der Unfallstellen zu berechnen.

Aus den 50 Unfallstellen mit den höchsten Unfallkosten wurden zunächst folgende Knotenpunkte als unfallauffällig ausgewählt:

- Die fünf Knotenpunkte mit der höchsten Anzahl der Unfälle mit Personenschaden U(P),

- die fünf Knotenpunkte mit den höchsten Gesamtunfallkosten und

- die fünf Knotenpunkte mit den höchsten Unfallkostenraten

Dabei gab es Überschneidungen, sodass insgesamt 13 Unfallstellen darüber begründet ausgewählt werden konnten. Weitere Kriterien für die verbleibenden zu wählenden Unfallstellen waren auffällige Unfallstrukturen (insbesondere Unfalltypen) und eine die Berücksichtigung der verschiedenen Knotenpunktformen.

Aus diesen Überlegungen heraus ergab sich folgende Auswahl der Knotenpunkte für die mikroskopische Analyse.

107 VBA = Visual Basic for Applications (VBA) ist eine Skriptsprache für die Steuerung von Abläufen u.a. in MS-Excel 
Tabelle 7-28: Knotenpunktauswahl der mikroskopischen Analyse

\begin{tabular}{|c|c|c|c|}
\hline & Stadt & Unfallstelle & Begründung der Auswahl \\
\hline 1 & Magdeburg & $\begin{array}{l}\text { KP Magdeburger Ring/ } \\
\text { Ebendorfer Chaussee }\end{array}$ & $\begin{array}{l}\text { - unter den Top } 5 \text { der höchsten Unfallkosten } \\
\text { - unter den Top } 5 \text { der höchsten } \\
\text { Unfallkostenraten }\end{array}$ \\
\hline 2 & Freiburg & $\begin{array}{l}\text { KP Opfinger Str./ Besanconallee/ } \\
\text { Betzenhauser Weg }\end{array}$ & - unter den Top 5 der höchsten Unfallkosten \\
\hline 3 & Hannover & $\begin{array}{l}\text { KP Tiergartenstr/ Lehrter Str./ } \\
\text { Am Tiergarten }\end{array}$ & $\begin{array}{l}\text { - unter den Top } 5 \text { der höchsten Unfallkosten } \\
\text { - unter den Top } 5 \text { der höchsten } \\
\text { Unfallkostenraten }\end{array}$ \\
\hline 4 & Dresden & $\begin{array}{l}\text { St Petersburger Str./ Haltestelle } \\
\text { Walpurgisstr. (Zufahrt Tiefgarage) }\end{array}$ & - unter den Top 5 der höchsten Unfallkosten \\
\hline 5 & Köln & $\begin{array}{l}\text { KP Aachener Str./ Haselbergstr. } \\
\text { (Gleisquerung) }\end{array}$ & - unter den Top 5 der höchsten Unfallkosten \\
\hline 6 & Leipzig & $\begin{array}{l}\text { KP Torgauer Str./ Zufahrt Gewerbegebiet } \\
\text { (toom-Baumarkt) }\end{array}$ & $\begin{array}{l}\text { - unter den Top } 5 \text { der höchsten } \\
\text { Unfallkostenraten }\end{array}$ \\
\hline 7 & Essen & $\begin{array}{l}\text { Altenessener Str. (Fußgängerquerung- } \\
\text { Elisabeth-Selbert-Str.) }\end{array}$ & $\begin{array}{l}\text { - unter den Top } 5 \text { der höchsten } \\
\text { Unfallkostenraten }\end{array}$ \\
\hline 8 & Gera & $\begin{array}{l}\text { Berliner Str. zw. Franz-Petrich-Str. und } \\
\text { Herderstr. }\end{array}$ & $\begin{array}{l}\text { - unter den Top } 5 \text { der höchsten } \\
\text { Unfallkostenraten }\end{array}$ \\
\hline 9 & Köln & KP Cäcilienstr./ Pipinstr./ Hohe Str. & $\begin{array}{l}\text { - unter den Top } 5 \text { mit den meisten Unfällen mit } \\
\text { Personenschaden }\end{array}$ \\
\hline 10 & Karlsruhe & KP Stephanplatz (Amalienstr./ Karlstr.) & $\begin{array}{l}\text { - unter den Top } 5 \text { mit den meisten Unfällen mit } \\
\text { Personenschaden }\end{array}$ \\
\hline 11 & München & KP Dachauer Str./ Landshuter Allee & $\begin{array}{l}\text { - unter den Top } 5 \text { mit den meisten Unfällen mit } \\
\text { Personenschaden }\end{array}$ \\
\hline 12 & Köln & $\begin{array}{l}\text { KP Chlodwigplatz (Karolingerring/ Ubierring/ } \\
\text { Bonner Str.) }\end{array}$ & $\begin{array}{l}\text { - unter den Top } 5 \text { mit den meisten Unfällen mit } \\
\text { Personenschaden }\end{array}$ \\
\hline 13 & Düsseldorf & KP Oststraße/ Graf-Adolf-Str. & $\begin{array}{l}\text { - unter den Top } 5 \text { mit den meisten Unfällen mit } \\
\text { Personenschaden }\end{array}$ \\
\hline 14 & Freiburg & KP Zähringer Str./ Hinterkirchstr. & $\begin{array}{l}\text { - } 5 \text { von } 6 \text { Unfällen vom Unfalltyp } 2 \text { (Abbiege- } \\
\text { Unfall) }\end{array}$ \\
\hline 15 & Köln & Gleisquerung Odenthaler Str. & $\begin{array}{l}\text { - } 6 \text { von } 6 \text { Unfällen vom Unfalltyp } 3 \text { (Einbiegen-/ } \\
\text { Kreuzen-Unfall) } \\
\text { - lichtsignalgeregelter Knotenpunkt }\end{array}$ \\
\hline 16 & Halle & $\begin{array}{l}\text { Große Steinstraße/ Joliot-Curie-Platz/ } \\
\text { Hansering }\end{array}$ & $\begin{array}{l}\text { - } 5 \text { von } 5 \text { Unfällen vom Unfalltyp } 3 \text { (Einbiegen-/ } \\
\text { Kreuzen-Unfall) } \\
\text { - Knotenpunkt mit vorfahrtregelnden } \\
\text { Verkehrszeichen }\end{array}$ \\
\hline 17 & Bremen & $\begin{array}{l}\text { KP Am Stern (Wachmannstr./ Herrmann- } \\
\text { Böse-Str.) }\end{array}$ & $\begin{array}{l}\text { - Kreisverkehrsplatz mit den zweithöchsten } \\
\text { Unfallkosten nach Köln KP Chlodwigplatz }\end{array}$ \\
\hline 18 & Leipzig & KP Ratzelstr./ Mannheimer Str. & - \\
\hline
\end{tabular}




\begin{tabular}{|l|l|l|l|}
\hline \multicolumn{1}{|c|}{ Stadt } & \multicolumn{1}{|c|}{ Unfallstelle } & \multicolumn{1}{c|}{ Begründung der Auswahl } \\
\hline 19 & Köln & $\begin{array}{l}\text { KP Gustav-Heinemann-Ufer/ } \\
\text { Schönhauser Str. }\end{array}$ & - \\
\hline 20 & Köln & KP Emdener Str./ Ivenshofweg & - \\
\hline 21 & Ludwigshaven & $\begin{array}{l}\text { KP Bleichstraße/ Seydlitzstraße (Paul- } \\
\text { Kleefoot-Platz) }\end{array}$ & $\begin{array}{l}- \text { Kreisverkehrsplatz mit den dritthöchsten } \\
\text { Unfallkosten als Ergänzung, da Köln KP } \\
\text { Chlodwigplatz von 2009 - 2011 im Umbau }\end{array}$ \\
\hline
\end{tabular}


Tabelle 7-29: untersuchte Unfallstellen der mikroskopischen Analyse

\begin{tabular}{|c|c|c|c|c|c|c|c|c|c|c|c|}
\hline $\begin{array}{l}\text { laufende } \\
\text { Nummer }\end{array}$ & 1 & 2 & 3 & 4 & 5 & 6 & 7 & 8 & 9 & 10 & 11 \\
\hline Stadt & $\begin{array}{c}\text { Magdeburg } \\
\text { (Sachsen- } \\
\text { Anhalt) }\end{array}$ & \begin{tabular}{|c|} 
Freiburg \\
(Baden- \\
Württemberg)
\end{tabular} & $\begin{array}{c}\text { Freiburg } \\
\text { (Baden- } \\
\text { Württemberg) }\end{array}$ & $\begin{array}{c}\text { Hannover } \\
\text { (Niedersachsen) }\end{array}$ & $\begin{array}{l}\text { Dresden } \\
\text { (Sachsen) }\end{array}$ & $\begin{array}{c}\text { Köln } \\
\text { (Nordrhein- } \\
\text { Westfalen) }\end{array}$ & $\begin{array}{c}\text { Köln } \\
\text { (Nordrhein- } \\
\text { Westfalen) }\end{array}$ & $\begin{array}{c}\text { Köln } \\
\text { (Nordrhein- } \\
\text { Westfalen) }\end{array}$ & $\begin{array}{c}\text { Köln } \\
\text { (Nordrhein- } \\
\text { Westfalen) } \\
\end{array}$ & $\begin{array}{c}\text { Köln } \\
\text { (Nordrhein- } \\
\text { Westfalen) }\end{array}$ & $\begin{array}{c}\text { Köln } \\
\text { (Nordrhein- } \\
\text { Westfalen) } \\
\end{array}$ \\
\hline Stadtteil & $\begin{array}{l}\text { Neustädter } \\
\text { See }\end{array}$ & Zähringen & Weingarten & Kirchrode & Altstadt & Altstadt & Dünnwald & Merkenich & Altstadt-Süd & Bayenthal & Ehrenfeld \\
\hline Lagebeschreibung & \begin{tabular}{|c|} 
KP \\
Magdeburger \\
Ring/ \\
Ebendorfer \\
Chaussee \\
\end{tabular} & $\begin{array}{c}\text { KP Zähringer } \\
\text { Str./ } \\
\text { Hinterkichstr. }\end{array}$ & $\begin{array}{c}\text { KP Opfinger Str./ } \\
\text { Besanconallee/ } \\
\text { Betzenhauser } \\
\text { Weg }\end{array}$ & $\begin{array}{l}\text { KP Tiergartenstr/ } \\
\text { Lehrter Str./ } \\
\text { Am Tiergarten }\end{array}$ & \begin{tabular}{|c|} 
St Petersburger \\
Str./ Haltestelle \\
Walpurgisstr. \\
(Zufahrt \\
Tiefgarage) \\
\end{tabular} & $\begin{array}{c}\text { KP } \\
\text { Cäcilienstr./ } \\
\text { Pipinstr./ } \\
\text { Hohe Str. }\end{array}$ & \begin{tabular}{|c|} 
Gleisquerung \\
Odenthaler \\
Str.
\end{tabular} & \begin{tabular}{|} 
KP Emdener \\
Str./ \\
Ivenshofweg
\end{tabular} & \begin{tabular}{|c|} 
KP \\
Chlodwigplatz \\
(Karolingerring/ \\
Ubierring/ \\
Bonner Str.) \\
\end{tabular} & \begin{tabular}{|c|} 
KP Gustav- \\
Heinemann- \\
Ufer/ \\
Schönhauser \\
Str.
\end{tabular} & \begin{tabular}{|l|} 
KP Aachener \\
Str./ \\
Haselbergstr. \\
(Gleisquerung)
\end{tabular} \\
\hline \multicolumn{12}{|l|}{$\begin{array}{c}\text { Abschnitt } \\
\text { (bei Strecken) }\end{array}$} \\
\hline $\begin{array}{c}\text { Art der } \\
\text { Verkehrsanlage }\end{array}$ & Knotenpunkt & Knotenpunkt & Knotenpunkt & Knotenpunkt & Knotenpunkt & Knotenpunkt & Knotenpunkt & Knotenpunkt & Knotenpunkt & Knotenpunkt & Knotenpunkt \\
\hline $\begin{array}{l}\text { Spezifikation der } \\
\text { Verkehrsanlage }\end{array}$ & $\begin{array}{l}\text { mit } \\
\text { Lichtsignal- } \\
\text { anlage }\end{array}$ & $\begin{array}{l}\text { mit vorfahrt- } \\
\text { regelnden } \\
\text { Verkehrs- } \\
\text { zeichen } \\
\end{array}$ & $\begin{array}{l}\text { mit Lichtsignal- } \\
\text { anlage }\end{array}$ & $\begin{array}{l}\text { mit Lichtsignal- } \\
\text { anlage }\end{array}$ & $\begin{array}{l}\text { mit Lichtsignal- } \\
\text { anlage }\end{array}$ & $\begin{array}{c}\text { aufgeweitet } \\
\text { mit } \\
\text { Lichtsignal- } \\
\text { anlage } \\
\end{array}$ & $\begin{array}{l}\text { mit } \\
\text { Lichtsignal- } \\
\text { anlage }\end{array}$ & $\begin{array}{l}\text { mit } \\
\text { Lichtsignal- } \\
\text { anlage }\end{array}$ & $\begin{array}{l}\text { Kreisverkehrs- } \\
\quad \text { platz }\end{array}$ & $\begin{array}{l}\text { mit } \\
\text { Lichtsignal- } \\
\text { anlage }\end{array}$ & \begin{tabular}{|c} 
mit vorfahrt- \\
regelnden \\
Verkehrs- \\
zeichen
\end{tabular} \\
\hline UK [€] & 1.394 .263 & 354.730 & 1.394 .263 & 1.375 .301 & 1.273 .785 & 329.064 & 354.730 & 418.322 & 633.612 & 1.115 .382 & 1.273 .785 \\
\hline $\begin{array}{c}\text { UKR } \\
\text { [€/1.000 Strab.- } \\
\text { Fz.] }\end{array}$ & 7.579 & 1.256 & 4.823 & 5.880 & 1.138 & 414 & 1.534 & 2.094 & 1.101 & 4.716 & 2.388 \\
\hline $\begin{array}{c}\text { Vzul } \\
{[\mathrm{km} / \mathrm{h}]}\end{array}$ & 50 & 50 & 50 & 50 & 50 & 50 & 50 & 50 & $30 / 50$ & 50 & 50 \\
\hline $\begin{array}{c}\text { DTV } \\
{[\mathrm{Kfz} / 24 \mathrm{~h}]}\end{array}$ & 12.000 & 37.600 & 12.900 & 7.000 & 25.500 & 28.470 & 7.720 & 2.450 & 13.370 & 48.350 & 40.000 \\
\hline $\begin{array}{c}\text { SV-Anteil } \\
{[\%]}\end{array}$ & 5 & 5 & 5 & 3 & 3 & $\approx 8,5$ & $\approx 7,7$ & $\approx 15,3$ & $\approx 16,5$ & $\approx 8,7$ & o.A. \\
\hline Quelle DTV & $\begin{array}{c}\text { Stadt } \\
\text { Magdeburg }\end{array}$ & Stadt Freiburg & Stadt Freiburg & Stadt Hannover & Stadt Dresden & Stadt Köln & Stadt Köln & Stadt Köln & Stadt Köln & Stadt Köln & \begin{tabular}{|c} 
Luftreinhalte- \\
plan $11 / 12$ \\
Stadt Köln \\
\end{tabular} \\
\hline $\begin{array}{l}\text { Erhebungs- } \\
\text { zeit DTV }\end{array}$ & 2009 & $07 / 2012$ & $07 / 2012$ & 09/2008 & $06 / 2012$ & $04 / 2014$ & 03/2011 & $06 / 2008$ & $07 / 2013$ & $04 / 2012$ & 09/2014 \\
\hline $\begin{array}{c}\text { DTV Straßenbahn } \\
\text { [Strab./ 24h] }\end{array}$ & 168 & 258 & 264 & 214 & 1.022 & 794 & 211 & 182 & 526 & 216 & 487 \\
\hline $\begin{array}{l}\text { Abschnitts- } \\
\text { länge [m] }\end{array}$ & 350 & 250 & 200 & 150 & 200 & 250 & 100 & 100 & 200 & 170 & 200 \\
\hline $\begin{array}{l}\text { laufende } \\
\text { Nummer }\end{array}$ & 12 & 13 & 14 & 15 & 16 & 17 & 18 & 19 & 20 & 21 & 22 \\
\hline Stadt & $\begin{array}{l}\text { Leipzig } \\
\text { (Sachsen) }\end{array}$ & $\begin{array}{l}\text { Leipzig } \\
\text { (Sachsen) }\end{array}$ & $\begin{array}{c}\text { Essen } \\
\text { (Nordrhein- } \\
\text { Westfalen) }\end{array}$ & $\begin{array}{c}\text { Gera } \\
\text { (Thüringen) }\end{array}$ & Bremen & \begin{tabular}{|c|}
$\begin{array}{c}\text { Karlsruhe } \\
\text { (Baden- } \\
\text { Württemberg) }\end{array}$ \\
\end{tabular} & $\begin{array}{l}\text { München } \\
\text { (Bayern) }\end{array}$ & $\begin{array}{c}\text { Düsseldorf } \\
\text { (Nordrhein- } \\
\text { Westfalen) } \\
\end{array}$ & $\begin{array}{c}\text { Halle } \\
\text { (Sachsen-Anhalt) }\end{array}$ & $\begin{array}{c}\text { Köln } \\
\text { (Nordrhein- } \\
\text { Westfalen) } \\
\end{array}$ & $\begin{array}{c}\text { Karlsruhe } \\
\text { (Baden- } \\
\text { Württemberg) }\end{array}$ \\
\hline Stadtteil & Nordost & Grünau & Altenessen & Innenstadt & Barkhof & $\begin{array}{c}\text { Innenstadt- } \\
\text { West }\end{array}$ & Moosach & Stadtmitte & Innenstadt & Riehl & Südweststadt \\
\hline Lagebeschreibung & \begin{tabular}{|c|} 
KP Torgauer \\
Str./ \\
Zufahrt Fa. \\
Massa-Haus \\
(Torgauer \\
Str.) \\
\end{tabular} & $\begin{array}{c}\text { KP Ratzelstr./ } \\
\text { Mannheimer } \\
\text { Str. }\end{array}$ & $\begin{array}{c}\text { Altenessener Str. } \\
\text { (Fußgängerqueru } \\
\text { ng-Elisabeth- } \\
\text { Selbert-Str.) }\end{array}$ & $\begin{array}{l}\text { Berliner Str. zw. } \\
\text { Franz-Petrich-Str. } \\
\text { und Herderstr. }\end{array}$ & $\begin{array}{c}\text { KP Am Stern } \\
\text { (Wachmannstr./ } \\
\text { Herrmann- } \\
\text { Böse-Str.) }\end{array}$ & \begin{tabular}{|c|} 
KP \\
Stephanplatz \\
(Amalienstr./ \\
Karlstr.)
\end{tabular} & $\begin{array}{l}\text { KP Dachauer } \\
\text { Str./ } \\
\text { Landshuter } \\
\text { Allee }\end{array}$ & $\begin{array}{c}\text { KP } \\
\text { Oststraße/ } \\
\text { Graf-Adolf- } \\
\text { Str }\end{array}$ & $\begin{array}{c}\text { Große } \\
\text { Steinstraße/ } \\
\text { Joliot-Curie- } \\
\text { Platz/ Hansering }\end{array}$ & $\begin{array}{c}\text { Amsterdamer } \\
\text { Str. }\end{array}$ & \begin{tabular}{|c|} 
unabhängiger \\
Bahnkörper
\end{tabular} \\
\hline $\begin{array}{c}\text { Abschnitt } \\
\text { (bei Strecken) }\end{array}$ & & & & & & & & & & $\begin{array}{c}\text { von } \\
\text { Innere } \\
\text { Kanalstr. } \\
- \\
\text { Johannes- } \\
\text { Müller-Str. }\end{array}$ & $\begin{array}{c}\text { von } \\
\text { Karl-Wolf-Weg } \\
- \\
\text { Siegfried- } \\
\text { Kühn-Str. }\end{array}$ \\
\hline $\begin{array}{c}\text { Art der } \\
\text { Verkehrsanlage }\end{array}$ & Knotenpunkt & Knotenpunkt & Knotenpunkt & Knotenpunkt & Knotenpunkt & Knotenpunkt & Knotenpunkt & Knotenpunkt & Knotenpunkt & Strecke & Strecke \\
\hline $\begin{array}{l}\text { Spezifikation der } \\
\text { Verkehrsanlage }\end{array}$ & $\begin{array}{l}\text { mit } \\
\text { Lichtsignal- } \\
\text { anlage }\end{array}$ & $\begin{array}{l}\text { mit vorfahrt- } \\
\text { regelnden } \\
\text { Verkehrs- } \\
\text { zeichen }\end{array}$ & $\begin{array}{l}\text { mit Lichtsignal- } \\
\text { anlage }\end{array}$ & $\begin{array}{l}\text { mit vorfahrt- } \\
\text { regelnden } \\
\text { Verkehrs- } \\
\text { zeichen }\end{array}$ & $\begin{array}{l}\text { Kreisverkehrs- } \\
\quad \text { platz }\end{array}$ & $\begin{array}{l}\text { mit } \\
\text { Lichtsignal- } \\
\text { anlage }\end{array}$ & $\begin{array}{c}\text { mit } \\
\text { Lichtsignal- } \\
\text { anlage }\end{array}$ & $\begin{array}{c}\text { mit } \\
\text { Lichtsignal- } \\
\text { anlage }\end{array}$ & $\begin{array}{l}\text { mit vorfahrt- } \\
\text { regelnden } \\
\text { Verkehrs- } \\
\text { zeichen }\end{array}$ & \begin{tabular}{|c|} 
3-streifiger/ \\
4-streifiger \\
Querschnitt \\
mit \\
besonderem \\
Bahnkörper in \\
Mittellage \\
\end{tabular} & \begin{tabular}{|c} 
unabhängiger \\
Bahnkörper
\end{tabular} \\
\hline UK [€] & 1.254 .822 & 1.134 .344 & 1.235 .860 & 1.115 .382 & 475.209 & 411.617 & 392.655 & 373.693 & 335.768 & 1.235 .860 & 278.881 \\
\hline $\begin{array}{c}\text { UKR } \\
\text { [€/1.000 Strab.- } \\
\text { Fz.] }\end{array}$ & 5.552 & 2.767 & 5.666 & 2.848 & 861 & 499 & 934 & 618 & 401 & 0 & 0 \\
\hline $\begin{array}{c}\begin{array}{c}\text { Vzul } \\
{[\mathrm{km} / \mathrm{h}]}\end{array} \\
\end{array}$ & 60 & $30 / 50$ & 50 & 50 & $30 / 50$ & $30 / 50$ & 60 & 50 & $20 / 50$ & 50 & - \\
\hline $\begin{array}{c}\text { DTV } \\
{[\mathrm{Kfz} / 24 \mathrm{~h}]}\end{array}$ & 26.922 & 10.938 & 0 & 5.350 & 25.000 & 0 & 35.000 & 49.955 & 13.996 & 21.350 & - \\
\hline $\begin{array}{c}\text { SV-Anteil } \\
{[\%]}\end{array}$ & 6 & 5 & 0 & 5 & o.A. & 0 & 3 & 3 & 2 & 3 & - \\
\hline Quelle DTV & Stadt Leipzig & Stadt Leipzig & $\begin{array}{l}\text { Kurzzeit- } \\
\text { zählung }\end{array}$ & Stadt Gera & Stadt Bremen & $\begin{array}{l}\text { Kurzzeit- } \\
\text { zählung }\end{array}$ & $\begin{array}{c}\text { Stadt } \\
\text { München }\end{array}$ & $\begin{array}{l}\text { Kurzzeit- } \\
\text { zählung }\end{array}$ & Stadt Halle & $\begin{array}{l}\text { Kurzzeit- } \\
\text { zählung }\end{array}$ & - \\
\hline $\begin{array}{l}\text { Erhebungs- } \\
\text { zeit DTV }\end{array}$ & $09 / 2008$ & 09/2008 & 09/2014 & $10 / 2013$ & $10 / 2002$ & 09/2014 & 2009 & 09/2014 & $06 / 2006$ & 09/2014 & - \\
\hline $\begin{array}{c}\text { DTV Straßenbahn } \\
\text { [Strab./ 24h] }\end{array}$ & 206 & 374 & 199 & 358 & 504 & 754 & 384 & 552 & 838 & 216 & 211 \\
\hline $\begin{array}{l}\text { Abschnitts- } \\
\text { länge [m] }\end{array}$ & 200 & 200 & 350 & 200 & - & 200 & 350 & 150 & 100 & 300 & 450 \\
\hline
\end{tabular}




\begin{tabular}{|c|c|c|c|c|c|c|c|c|c|c|}
\hline $\begin{array}{l}\text { laufende } \\
\text { Nummer }\end{array}$ & 23 & 24 & 25 & 26 & 27 & 28 & 29 & 30 & 31 & 32 \\
\hline Stadt & $\begin{array}{l}\text { Düsseldorf } \\
\text { (Nordrhein- } \\
\text { Westfalen) }\end{array}$ & $\begin{array}{c}\text { Halle } \\
\text { (Sachsen- } \\
\text { Anhalt) } \\
\end{array}$ & $\begin{array}{l}\text { Frankfurt/ Main } \\
\text { (Hessen) }\end{array}$ & $\begin{array}{c}\text { Bonn } \\
\text { (Nordrhein- } \\
\text { Westfalen) }\end{array}$ & $\begin{array}{c}\text { Stuttgart } \\
\text { (Baden- } \\
\text { Württemberg) } \\
\end{array}$ & \begin{tabular}{|c|}
$\begin{array}{c}\text { Stuttgart } \\
\text { (Baden- } \\
\text { Württemberg) }\end{array}$ \\
\end{tabular} & $\begin{array}{c}\text { Cottbus } \\
\text { (Brandenburg) }\end{array}$ & \begin{tabular}{|c|} 
Erfurt \\
(Thüringen)
\end{tabular} & $\begin{array}{c}\begin{array}{c}\text { Heidelberg } \\
\text { (Baden- } \\
\text { Württemberg ) }\end{array} \\
\end{array}$ & \begin{tabular}{|c}
$\begin{array}{c}\text { Ludwigshafen } \\
\text { (Rheinland- } \\
\text { Pfalz) }\end{array}$ \\
\end{tabular} \\
\hline Stadtteil & Pempelfort & Neustadt & Ostend & Zentrum & Möhringen & Bergheim & Saspow & Rieth & Bergheim & Süd \\
\hline Lagebeschreibung & $\begin{array}{c}\text { Oberkasseler } \\
\text { Brücke/ } \\
\text { Hofgarten- } \\
\text { rampe }\end{array}$ & $\begin{array}{c}\text { An der } \\
\text { Magistrale }\end{array}$ & Hanauer Landstr. & Am Hauptbahnhof & Probststr. & Engelbergstr. & $\begin{array}{l}\text { Gerhart- } \\
\text { Hauptmann- } \\
\text { Str. }\end{array}$ & $\begin{array}{c}\text { Warschauer } \\
\text { Str., } \\
\text { Vilniuser Str. }\end{array}$ & $\begin{array}{l}\text { Eppelheimer } \\
\text { Straße }\end{array}$ & $\begin{array}{c}\text { KP } \\
\text { Bleichstraße/ } \\
\text { Seydlitzstraße } \\
\text { (Paul- } \\
\text { Kleefoot- } \\
\text { Platz) } \\
\end{array}$ \\
\hline $\begin{array}{c}\text { Abschnitt } \\
\text { (bei Strecken) }\end{array}$ & $\begin{array}{c}\text { von } \\
\text { Luegplatz } \\
- \\
\text { Maximilian- } \\
\text { Weyhe-Allee. }\end{array}$ & $\begin{array}{c}\text { von } \\
\text { Zur Saaleaue } \\
- \\
\text { Gimritzer } \\
\text { Damm } \\
\text { (Rennbahn- } \\
\text { kreuz) }\end{array}$ & $\begin{array}{c}\text { von } \\
\text { Intzestr. } \\
- \\
\text { Schwedlerstr. }\end{array}$ & $\begin{array}{c}\text { von } \\
\text { Busbahnhof } \\
- \\
\text { Thomas-Mann- } \\
\text { Str. }\end{array}$ & $\begin{array}{c}\text { von } \\
\text { Rembrandtstr. } \\
- \\
\text { Balinger Str. }\end{array}$ & $\begin{array}{c}\text { von } \\
\text { Salamander- } \\
\text { weg } \\
- \\
\text { Schildkröten- } \\
\text { weg }\end{array}$ & $\begin{array}{c}\text { von } \\
\text { Nordring } \\
- \\
\text { Am Großen } \\
\text { Spreewehr }\end{array}$ & \begin{tabular}{|c|} 
von KP \\
Warschauer \\
Str./Berliner \\
Str. \\
- \\
KP Vilniuser \\
Str./ \\
Mainzer Str. \\
\end{tabular} & \begin{tabular}{|c|} 
von \\
KP Eppelheimer \\
Str./ Henkel- \\
Teroson-Straße \\
- \\
Eppelheimer \\
Str./ Czernyring
\end{tabular} & \\
\hline $\begin{array}{c}\text { Art der } \\
\text { Verkehrsanlage }\end{array}$ & Strecke & Strecke & Strecke & Strecke & Strecke & Strecke & Strecke & Strecke & Strecke & Knotenpunkt \\
\hline $\begin{array}{l}\text { Spezifikation der } \\
\text { Verkehrsanlage }\end{array}$ & $\begin{array}{c}\text { 3-streifiger/ } \\
\text { 4-streifiger } \\
\text { Querschnitt } \\
\text { mit } \\
\text { besonderem } \\
\text { Bahnkörper } \\
\text { in Mittellage }\end{array}$ & \begin{tabular}{|c|} 
3-streifiger/ \\
4-streifiger \\
Querschnitt \\
mit \\
besonderem \\
Bahnkörper in \\
Mittellage
\end{tabular} & $\begin{array}{l}\text { 3-streifiger/ } \\
\text { 4-streifiger } \\
\text { Querschnitt mit } \\
\text { besonderem } \\
\text { Bahnkörper in } \\
\text { Mittellage }\end{array}$ & $\begin{array}{l}\text { 2-streifiger } \\
\text { Querschnitt mit } \\
\text { straßen- } \\
\text { bündigen } \\
\text { Bahnkörper in } \\
\text { Mittellage }\end{array}$ & $\begin{array}{l}\text { unabhängiger } \\
\text { Bahnkörper }\end{array}$ & $\begin{array}{c}\text { besonderer } \\
\text { Bahnkörper in } \\
\text { Seitenlage }\end{array}$ & \begin{tabular}{|c|} 
besonderer \\
Bahnkörper in \\
Seitenlage
\end{tabular} & \begin{tabular}{c|} 
\\
besonderer \\
Bahnkörper \\
in Seitenlage
\end{tabular} & $\begin{array}{c}\text { 3-streifiger/ } \\
\text { 4-streifiger } \\
\text { Querschnitt mit } \\
\text { straßenbündigen } \\
\text { Bahnkörper in } \\
\text { Mittellage }\end{array}$ & $\begin{array}{l}\text { Kreisverkehrs- } \\
\text { platz }\end{array}$ \\
\hline UK $[€]$ & 1.115 .382 & 278.881 & 75.849 & 56.887 & 158.403 & 278.881 & 1.235 .860 & 37.925 & 37.925 & 297.844 \\
\hline $\begin{array}{c}\text { UKR } \\
{[€ / 1.000 \text { Strab.-Fz.] }}\end{array}$ & 0 & 0 & 0 & 0 & 0 & 0 & 0 & 0 & 0 & 955 \\
\hline $\begin{array}{c}\text { Vzul } \\
{[\mathrm{km} / \mathrm{h}]}\end{array}$ & 50 & 60 & 50 & 50 & 30 & 50 & 50 & 50 & 50 & 20 \\
\hline $\begin{array}{c}\text { DTV } \\
{[\mathrm{Kfz} / 24 \mathrm{~h}]}\end{array}$ & 20.238 & 19.924 & 29.102 & 7.800 & 3.760 & 11.798 & 6.466 & 6.323 & 8.545 & o.A. \\
\hline $\begin{array}{c}\text { SV-Anteil } \\
{[\%]}\end{array}$ & 4 & 4 & 4 & 3 & 1 & 1 & 2 & 3 & o.A. & o.A. \\
\hline Quelle DTV & $\begin{array}{c}\text { Stadt } \\
\text { Düsseldorf }\end{array}$ & Stadt Halle & Stadt Frankfurt & $\begin{array}{l}\text { Kurzzeit- } \\
\text { zählung }\end{array}$ & Stadt Stuttgart & $\begin{array}{c}\text { Stadt } \\
\text { Stuttgart }\end{array}$ & Stadt Cottbus & $\begin{array}{l}\text { Kurzzeit- } \\
\text { zählung }\end{array}$ & $\begin{array}{c}\text { Kurzzeit- } \\
\text { zählung Stadt } \\
\text { Heidelberg }\end{array}$ & - \\
\hline $\begin{array}{l}\text { Erhebungs- } \\
\text { zeit DTV }\end{array}$ & 09/2013 & 03/2012 & $08 / 2013$ & 09/2014 & 04/2011 & 07/2007 & 03/2010 & 08/2014 & $02 / 2013$ & - \\
\hline $\begin{array}{c}\text { DTV Straßenbahn } \\
\text { [Strab./ 24h] }\end{array}$ & 571 & 590 & 288 & 454 & 758 & 266 & 127 & 372 & 202 & 312 \\
\hline $\begin{array}{l}\text { Abschnitts- } \\
\text { länge }[\mathrm{m}]\end{array}$ & 400 & 450 & 1.200 & 400 & 400 & 330 & 640 & 650 & 250 & - \\
\hline
\end{tabular}

\subsection{Datengrundlage, Ortsbesichtigungen, Sicherheitsaudits, Datenauswertung und Dokumentation}

Die in der mikroskopischen Analyse angewandte systematische Untersuchung gleicht den beiden Verfahren "Örtlichen Unfalluntersuchung" und "Bestandsaudit". Beide Verfahren dienen als Elemente des Verkehrssicherheitsmanagements dem Zweck, Defizite der Infrastruktur zu erkennen und durch geeignete Maßnahmen zu beseitigen bzw. zu relativieren.

Die Örtliche Unfalluntersuchung ist seit Jahren ein wichtiger Bestandteil der Verkehrssicherheitsarbeit. Auf Grundlage der VwV-StVO zu §44 StVO werden bei der Örtlichen Unfalluntersuchung mittels Unfalltypenkarten unfallauffällige Stellen (Unfallhäufungsstellen und Unfallhäufungslinien) erkannt und auf Basis von Unfalldiagrammen und Unfalllisten mögliche unfallbegünstigende Faktoren herausgearbeitet. Im Rahmen einer Ortsbesichtigung werden Maßnahmen entwickelt um die Zahl und die Schwere der Unfälle zu reduzieren.

Für das Bestandsaudit gibt es im Gegensatz zur Örtlichen Unfalluntersuchung und dem bereits bestehenden Audit für Planungs- und Entwurfsunterlagen noch keine einheitlichen Prozeduren. Dennoch bestehen für Bestandsaudit Erfahrungen durch sog. Pilotaudits. Auch beim Bestandsaudit ist eine Ortsbegehung und die Aufnahme von Defiziten ein wesentlicher Bestandteil der Untersuchung. Vorrangig unterscheidet sich das Bestandsaudit von der Örtlichen Unfalluntersuchung in der Auswahl der zu untersuchenden Örtlichkeiten: Das Bestandsaudit sollte anlassbezogen sein, z.B. auf Basis einer Netzanalyse oder vor Um- und Ausbau von Straßen statt 
auf Basis fester Grenzwerte der Unfallhäufungen. Die Defizite des Bestandsaudits werden in einem Auditbericht festgehalten und sind i.A. als Prüfaufträge formuliert. Die Formulierung eines Defizits als Prüfauftrag hat die Vorteile, dass

- dem Baulastträger in der Abwägung zwischen der Verkehrssicherheit und weiteren verkehrsplanerischen Belangen (z.B. Umwelt, Kosten, Städtebau) ein Handlungsspielraum gelassen wird,

- mögliche Defizite im Anschluss des Bestandsaudit einer genaueren Überprüfung durch weitergehende Erhebungen unterzogen werden können, ohne dass während der Auditierung durch den Auditor eigene Berechnungen und tiefgreifende Untersuchungen angestellt werden müssen,

- die Akzeptanz des Audits bei Entscheidungsträgern erhöht wird.

Für die mikroskopische Analyse im Rahmen des Forschungsvorhabens wurden Bestandteile der Örtlichen Unfalluntersuchung nach M Uko und des Bestandsaudits nach den ESAS zusammengeführt. Zunächst wurden die Unfälle der Unfallstellen als Unfalltypenkarten kartiert. Ergänzt um die Unfalllisten der vorliegenden Unfälle mit Personenschaden der Jahre 2009 bis 2011, wurden vor der Ortsbegehung Hinweise auf mögliche unfallbegünstigende Faktoren ersichtlich.

Während der Ortsbesichtigung wurden die erkannten Defizite fotodokumentiert und anschließend in Analogie zu einem Audit als Prüfauftrag formuliert.

Die Ortsbesichtigungen fanden an mittleren Werktagen außerhalb der Ferienzeiten statt. Lagen keine Verkehrsmengen des Kfz-Verkehrs vor, wurden im Rahmen der Ortsbesichtigungen Kurzzeitzählungen nach $\mathrm{HBS}^{108}$ durchgeführt, die eine Hochrechnung auf die durchschnittlich täglichen Verkehrsstärken (DTV) ermöglichten.

Wie in der gesamten bisherigen Untersuchung liegt der Schwerpunkt der mikroskopischen Analyse auf Defiziten der Straßenverkehrsinfrastruktur aus denen sich Unfälle mit Beteiligung von Straßenbahnen entwickeln können. Defizite die kein Konfliktpotential mit Straßenbahnen erkennen ließen, werden in der nachfolgenden Zusammenstellung der Ergebnisse unberücksichtigt gelassen. Es werden jedoch auch in Analogie zum Audit und der Örtlichen Unfalluntersuchung Defizite aufgeführt, die offensichtlich Konflikte mit Straßenbahnen entstehen lassen, trotzdessen dass nicht zwangsläufig ein Unfall in den betrachteten drei Jahren darauf zurückzuführen war.

\subsection{Ergebnisse der mikroskopischen Analyse}

Die Ergebnisse der makroskopischen Analyse sind Defizite der Straßeninfrastruktur, die aus den Ortsbegehungen heraus erkannt wurden.

Die Dokumentation und Darstellung der Erkenntnisse der Ortsbesichtigungen erfolgt in Dossierbriefen (Steckbriefen) mit folgenden wesentlichen Inhalten:

- Datenblatt mit Unfalltypenkarte und Unfalldaten sowie Informationen zur Unfallstelle

- Sicherheitsdefizite (Formulierung als Prüffragen in Analogie zu den Sicherheitsaudits)

- Fotodokumentation

108 [HBS 2009], S. 2-15ff. 
Die Dossierbriefe befinden sich vollständig in einem gesonderten Anlagenband (Anlagenband Teil 2, Dossierbriefe).

Im Folgenden werden beispielhaft wesentliche, wiederkehrend erkannte Defizite vorgestellt:

Tabelle 7-30: $\quad$ ausgewählte, wiederkehrende Defizite in Bildern

Mangelnde Erkennbarkeit und Begreifbarkeit der Lichtsignalsteuerung
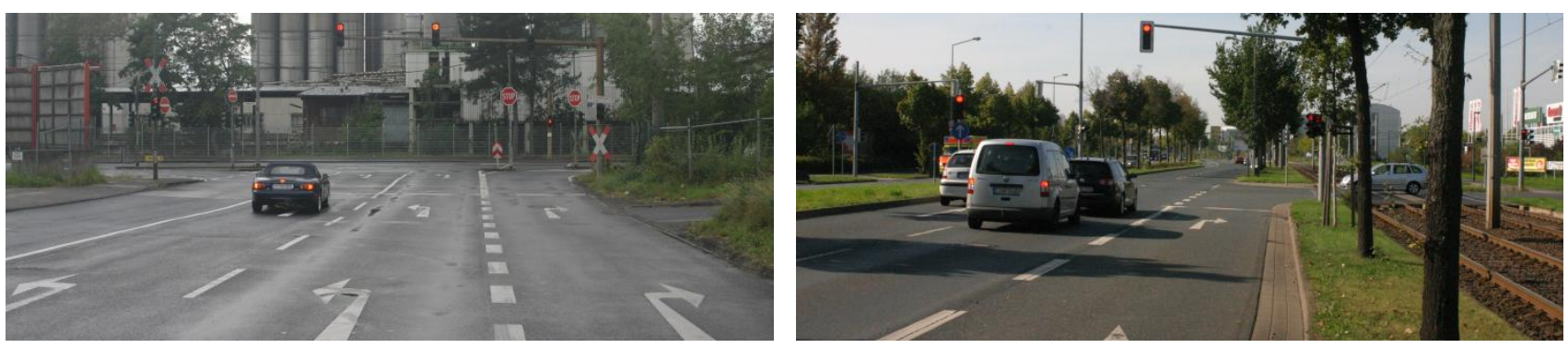

Keine eindeutige Signalisierung der Straßenbahn an signalisierten Querungssstellen z.B. mittels den Signalbildern F0 (Halt) und F1 (Fahrt freigegeben)
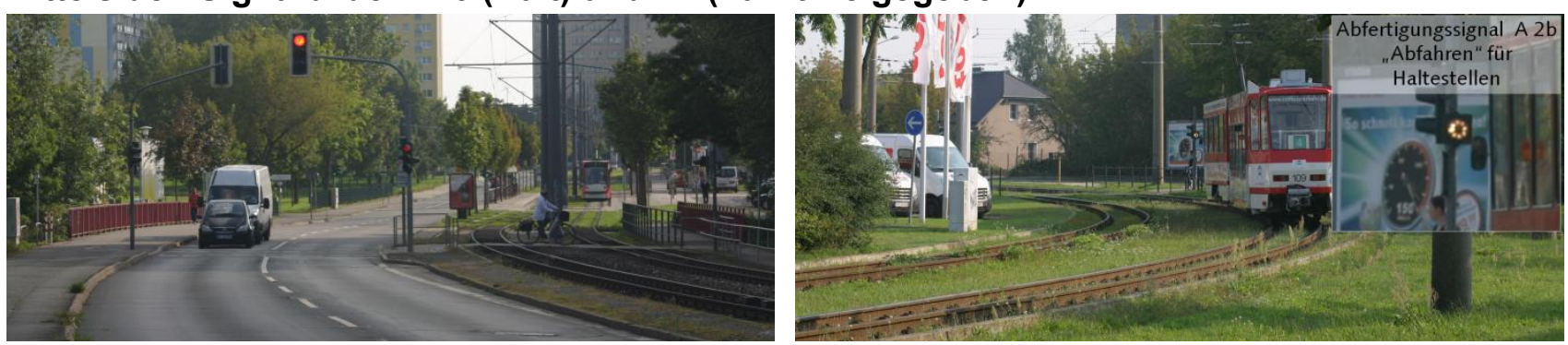

Fehlende Signalisierung für Kfz trotz mangelnder Erkennbarkeit, Begreifbarkeit, Übersichtlichkeit und/ oder einer relevanten Knotenstrombelastung
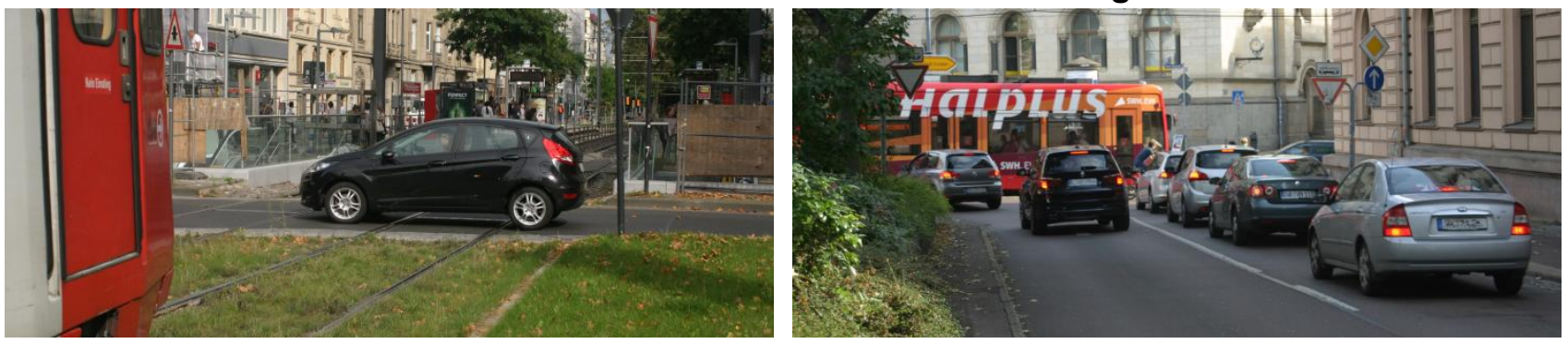

Fehlende Querungshilfe bei ausgeprägtem Querungsbedarf bzw. Querungshilfen außerhalb der Fußgängerquerungslinien
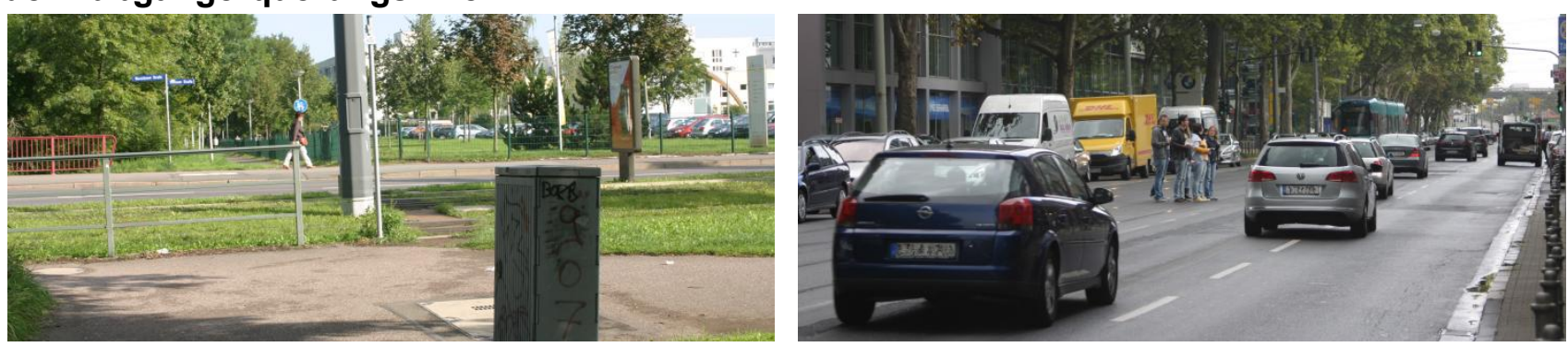
Zugänge nicht an beiden Bahnsteigenden (bzw. umwegebehaftete Anbindung)
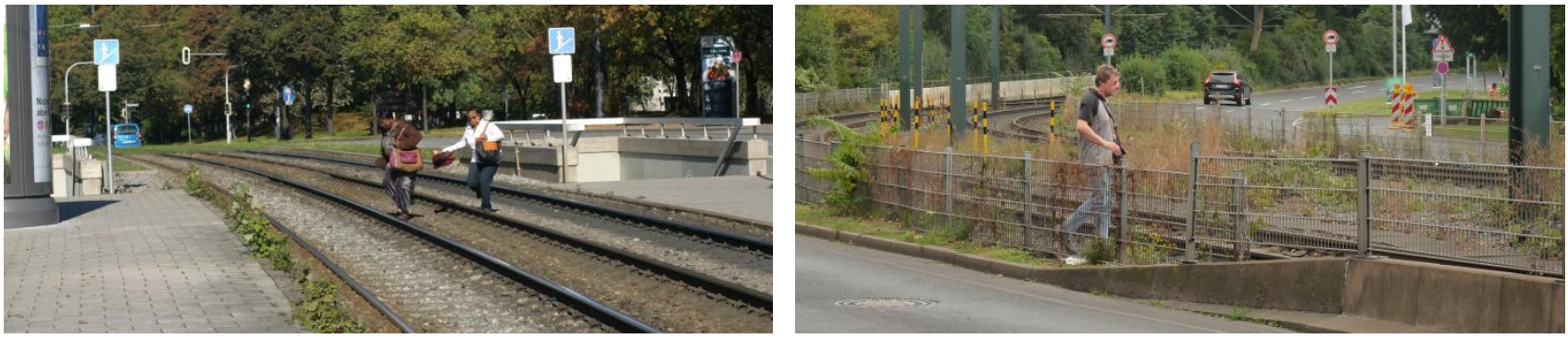

Nicht zulässige Fahrbeziehungen mit konfligierenden Strömen bei wiederrechtlichem Befahren bzw. unzureichende Anzahl zulässiger Wendemöglichkeiten bei besonderen Bahnkörpern in Mittellage
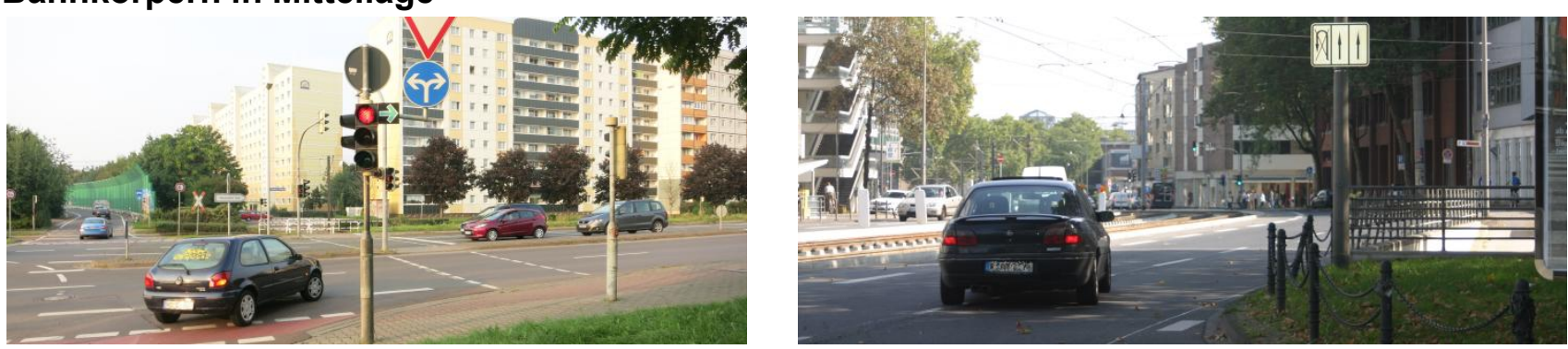

Mangelnde Erkennbarkeit der Gleisquerung und Schienenfahrzeuge (fehlende Sichtfelder)
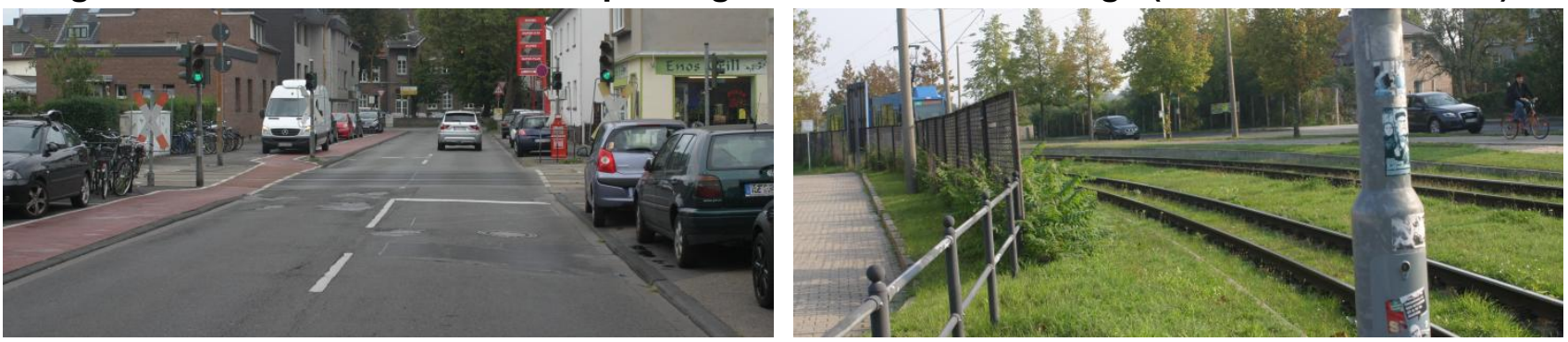

Fehlende zusätzliche Sicherungseinrichtungen (Umlaufgitter, akustische Signalgeber) im Zuge von Radwegen und unübersichtlichen Querungsstellen
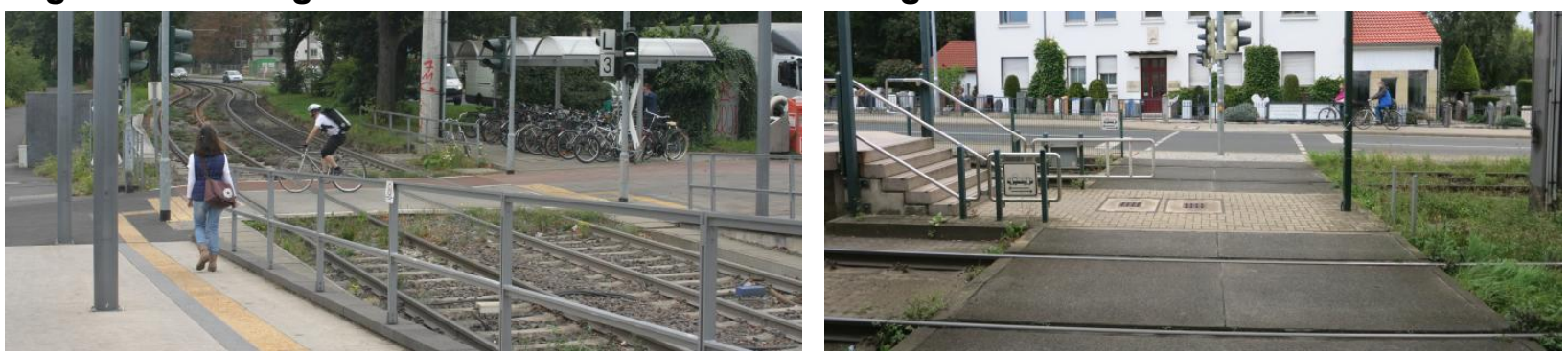

Fehlende bauliche Trennung von nur durch die Straßenbahn zu befahrene Bahnkörper
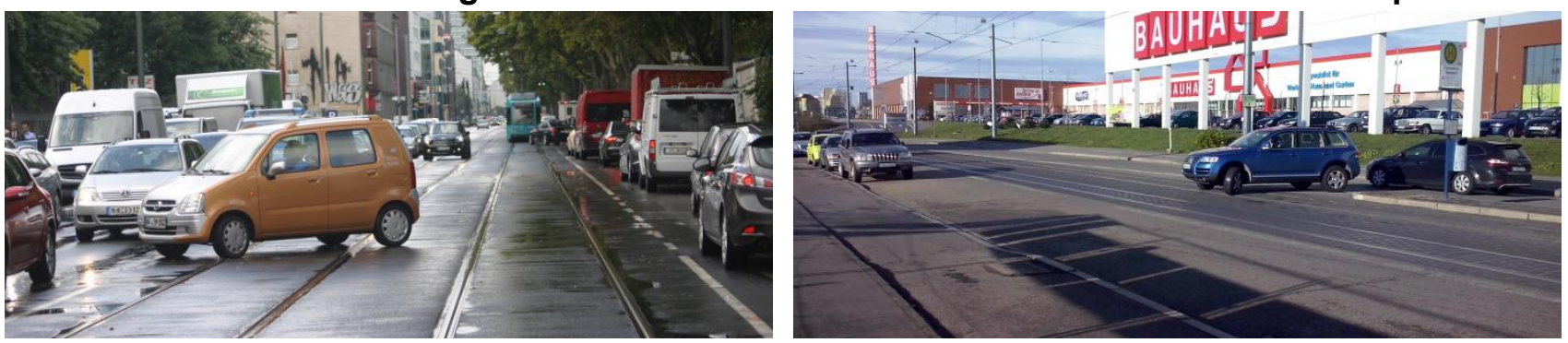
Bei den Ortsbegehungen hat sich gezeigt, dass ausnahmslos alle ausgewählten unfallauffälligen Örtlichkeiten durch zum Teil erhebliche Sicherheitsdefizite der Infrastruktur gekennzeichnet waren, die von geltenden sicherheitsrelevanten Einträgen der Regelwerke abwichen.

Aufgrund der zahlreichen Sicherheitsdefizite war es möglich den einzelnen typisierten Verkehrsanlagen charakteristische Defizite zuzuweisen.

Im Folgenden werden erkannte Defizite nach Knotenpunktform bzw. Querschnittstyp und weiter untergliedert nach Art der Verkehrsteilnahme zusammengefasst. Dabei werden insbesondere Defizite aufgeführt, die einer gewissen Allgemeingültigkeit der entsprechenden Verkehrsanlage zuzurechnen sind und in ähnlicher Form mehrfach in den Dossierbriefen als Defizit benannt wurden. Allgemeine Defizite, die praktisch auf alle Verkehrsanlagen zutreffen würden (bspw. mangelnde Erkennbarkeit von überalterter Fahrbahnmarkierung), bleiben in diesem Kapitel unerwähnt und können als Defizite für die entsprechende Verkehrsanlage den Dossierbriefen entnommen werden.

Neben einer Reihe an charakteristischen, unfallbegünstigenden Umständen, die in den Regelwerken nicht oder nur unzureichend benannt und thematisiert wurden, ist die Mehrzahl der erkannten Defizite darauf zurückzuführen, dass Abweichungen von Regelwerken und Richtlinien festzustellen waren.

Für die folgenden, aufgeführten Defizite befindet sich in Anlage A.8 beispielhaft je ein Foto zur Veranschaulichung. 
Tabelle 7-31: Defizite nach Art der Verkehrsanlage und Art der Verkehrsteilnahme, Knotenpunkte

\begin{tabular}{|c|c|c|}
\hline \multicolumn{3}{|l|}{ Knotenpunkte } \\
\hline \multirow{4}{*}{$\begin{array}{l}\text { Knotenpunkte } \\
\text { mit } \\
\text { Lichtsignalanlage }\end{array}$} & $\begin{array}{l}\text { Fuß- } \\
\text { gänger }\end{array}$ & $\begin{array}{l}\text { - Fehlende Sichtfelder im Fall von Ausfall der Lichtsignalanlage und zur } \\
\text { Erhöhung der Wahrnehmbarkeit bevorrechtigter Schienenfahrzeuge } \\
\text { - Keine umwegefreie gradlinige Führung über Fußgängerfurten in jedem } \\
\text { Knotenpunktarm } \\
\text { - Grundsatz des durchgängigen Betriebs Tag und Nacht nicht beachtet } \\
\text { - Eingeschränkte Sicht auf Signalgeber } \\
\text { - Unterschiedliche Freigabezeit hintereinanderliegender Furten }\end{array}$ \\
\hline & $\begin{array}{l}\text { Rad- } \\
\text { fahrer }\end{array}$ & $\begin{array}{l}\text { - Fehlende Sichtfelder im Fall von Ausfall der Lichtsignalanlage und zur } \\
\text { Erhöhung der Wahrnehmbarkeit bevorrechtigter Schienenfahrzeuge } \\
\text { - Fehlende zusätzliche Signalgeber für den Radverkehr bei Führung des } \\
\text { Radverkehrs auf der Fahrbahn und gemeinsamer Signalisierung mit } \\
\text { dem Kfz-Verkehr }\end{array}$ \\
\hline & $\mathrm{Kfz}$ & $\begin{array}{l}\text { - Fehlende Sichtfelder im Fall von Ausfall der Lichtsignalanlage und zur } \\
\text { Erhöhung der Wahrnehmbarkeit bevorrechtigter Schienenfahrzeuge } \\
\text { - Schlechte Erkennbarkeit und Begreifbarkeit der Lichtsignalsteuerung } \\
\text { - Grundsatz des durchgängigen Betriebs Tag und Nacht nicht beachtet } \\
\text { - Fehlende Erkennbarkeit von signalisierten Gleisquerungen außerhalb } \\
\text { von Verkehrsknotenpunkten } \\
\text { - Fehlender Zeitvorsprung oder räumlicher Vorsprung für Straßenbahnen } \\
\text { im Fall von Missachtung von Fahrtrichtungsgeboten } \\
\text { - Keine/ ungeeignete Unterstützung von Fahrtrichtungsgeboten } \\
\text { - Unzureichende Anzahl an zulässigen Wendemöglichkeiten bei baulich } \\
\text { getrennten Bahnkörpern mit anschließendem Verbot des Wendens } \\
\text { oder Abbiegens am Knotenpunkt } \\
\text { - Eingeschränkte Sicht auf Signalgeber } \\
\text { - Unvollständige Signalisierung }\end{array}$ \\
\hline & $\begin{array}{l}\text { Halte- } \\
\text { stelle }\end{array}$ & $\begin{array}{l}\text { - Fehlende geschwindigkeitsdämpfende Elemente für Radfahrer an } \\
\text { Rampen von Hochbahnsteigen }\end{array}$ \\
\hline \multirow{4}{*}{$\begin{array}{l}\text { Knotenpunkte } \\
\text { ohne } \\
\text { Lichtsignalanlage }\end{array}$} & $\begin{array}{l}\text { Fuß- } \\
\text { gänger }\end{array}$ & - Keine geeigneten Überquerungsanlagen über bevorrechtigte Zufahrten \\
\hline & $\begin{array}{l}\text { Rad- } \\
\text { fahrer }\end{array}$ & $\begin{array}{l}\text { - Keine geeigneten Überquerungsanlagen über bevorrechtigte Zufahrten } \\
\text { - Schlechte Erkennbarkeit, Begreifbarkeit, Übersichtlichkeit, } \\
\text { Einheitlichkeit und Befahrbarkeit der Radverkehrsanlagen } \\
\text { - Fehlende Möglichkeit des Linksabbiegens für Radfahrer }\end{array}$ \\
\hline & $\mathrm{Kfz}$ & $\begin{array}{l}\text { - Fehlende Signalisierung trotz mangelnder Erkennbarkeit, } \\
\text { Begreifbarkeit, Übersichtlichkeit, Einheitlichkeit und/oder einer } \\
\text { relevanter Knotenstrombelastung }\end{array}$ \\
\hline & $\begin{array}{l}\text { Halte- } \\
\text { stelle }\end{array}$ & \\
\hline
\end{tabular}




\begin{tabular}{|c|c|c|}
\hline \multicolumn{1}{|c|}{ Knotenpunkte (Fortsetzung) } \\
\hline \multirow{4}{*}{$\begin{array}{c}\text { Kreisverkehrs- } \\
\text { Plätze }\end{array}$} & $\begin{array}{c}\text { Fuß- } \\
\text { gänger }\end{array}$ & $\begin{array}{c}\text { - Überquerungsstellen zu weit von der Kreisfahrbahn abgesetzt } \\
\text { - Einheitlichkeit der Knotenpunktzu- und Ausfahrten ist nicht gegeben }\end{array}$ \\
\cline { 2 - 4 } & $\begin{array}{c}\text { Rad- } \\
\text { fahrer }\end{array}$ & $\begin{array}{c}\text { - Keine signaltechnische Sicherung bei Führung von Straßenbahnen } \\
\text { über die Kreisfahrbahn }\end{array}$ \\
\cline { 2 - 4 } & Kfz & $\begin{array}{c}\text { - Keine signaltechnische Sicherung bei Führung von Straßenbahnen } \\
\text { über die Kreisfahrbahn } \\
\text { - Überschreitung der Kapazität des Kfz-Verkehrs mit Rückstau auf die } \\
\text { Gleisquerung in der Kreisfahrbahn }\end{array}$ \\
\cline { 2 - 4 } & $\begin{array}{c}\text { Halte- } \\
\text { stelle }\end{array}$ & \\
\hline
\end{tabular}

Tabelle 7-32:

Defizite nach Art der Verkehrsanlage und Art der Verkehrsteilnahme, Strecken

\begin{tabular}{|c|c|c|}
\hline \multicolumn{3}{|l|}{ Strecken } \\
\hline \multirow{4}{*}{$\begin{array}{l}\text { Besondere } \\
\text { Bahnkörper in } \\
\text { Mittellage }\end{array}$} & $\begin{array}{l}\text { Fuß- } \\
\text { gänger }\end{array}$ & $\begin{array}{l}\text { - Unzureichende Aufstellflächen zwischen Gleisbereich und Fahrbahn } \\
\text { bei linearem Überquerungsbedarf } \\
\text { - Fehlende Überquerungsanlage bei ausgeprägtem Überquerungsbedarf } \\
\text { - Lage der Querungshilfe außerhalb der Fußgängerüberquerungslinien } \\
\text { - Bei Signalregelung im Gleisbereich keine Unabhängigkeit von der } \\
\text { Signalisierung der Fahrbahnfurten } \\
\text { - Unterschiedliche Freigabezeit hintereinanderliegender Furten } \\
\text { - Keine Eindeutige Signalisierung der Straßenbahn in Verbindung mit } \\
\text { den Signalbildern F0 (Halt) und F1 (Fahrt freigegeben) an signalisierten } \\
\text { Querungsstellen } \\
\text { - Lange Wartezeiten/ Sperrzeiten für Fußgänger }\end{array}$ \\
\hline & $\begin{array}{l}\text { Rad- } \\
\text { fahrer }\end{array}$ & $\begin{array}{l}\text { - Besonderer Bahnkörper von Straßenbahnen ist zu Lasten } \\
\text { ausreichender Flächen für andere Nutzungsansprüche } \\
\text { - Keine Verhinderung der Mitbenutzung von ÖPNV-Fahrstreifen mit } \\
\text { Gleisen durch Radfahrer }\end{array}$ \\
\hline & $\mathrm{Kfz}$ & $\begin{array}{l}\text { - Besondere Bahnkörper sind nicht baulich durch Borde von der } \\
\text { Fahrbahn abgegrenzt }\end{array}$ \\
\hline & $\begin{array}{l}\text { Halte- } \\
\text { stelle }\end{array}$ & $\begin{array}{l}\text { - Umwegebehaftete Anbindung der Haltestelle für Fußgänger } \\
\text { - Lage der Haltestelle gewährleistet keine sichere Erreichbarkeit für } \\
\text { Fahrgäste } \\
\text { - Fehlende zusätzliche Sicherungseinrichtungen (Umlaufgitter, } \\
\text { zusätzliche Signalgeber in geringer Höhe, akustische Signale) bei } \\
\text { unübersichtlichen Querungsstellen, gradliniger Führung oder bei } \\
\text { hohem Fußgänger- und Radverkehrsaufkommen } \\
\text { - gradlinige Führung querender Fußgänger und Radfahrer über die } \\
\text { Haltestelleninsel } \\
\text { - Freigabezeiten der Fußgängerfurten als Haltestellenzugang nicht auf } \\
\text { einfahrende ÖPNV-Fahrzeuge abgestimmt } \\
\text { - Keine Anrampung der Haltestelleninsel zur Überquerungsstelle }\end{array}$ \\
\hline
\end{tabular}




\begin{tabular}{|c|c|c|}
\hline \multicolumn{3}{|c|}{ Strecken (Fortsetzung) } \\
\hline \multirow{4}{*}{$\begin{array}{l}\text { Besondere } \\
\text { Bahnkörper in } \\
\text { Seitenlage }\end{array}$} & $\begin{array}{l}\text { Fuß- } \\
\text { gänger }\end{array}$ & $\begin{array}{l}\text { - Fehlende Überquerungsanlage bei ausgeprägtem Überquerungsbedarf } \\
\text { - Lage der Querungshilfe außerhalb der Fußgängerüberquerungslinien } \\
\text { - Unterschiedliche Freigabezeit hintereinanderliegender Furten } \\
\text { - Keine eindeutige Signalisierung der Straßenbahn in Verbindung mit } \\
\text { den Signalbildern F0 (Halt) und F1 (Fahrt freigegeben) an signalisierten } \\
\text { Querungsstellen } \\
\text { - Lange Wartezeiten/ Sperrzeiten für Fußgänger }\end{array}$ \\
\hline & $\begin{array}{l}\text { Rad- } \\
\text { fahrer }\end{array}$ & $\begin{array}{l}\text { - Fehlende zusätzliche Sicherungseinrichtungen (Umlaufgitter, } \\
\text { zusätzliche Signalgeber in geringer Höhe, akustische Signale) bei } \\
\text { unübersichtlichen Querungsstellen, gradliniger Führung oder bei } \\
\text { hohem Fußgänger- und Radverkehrsaufkommen } \\
\text { - fehlende Signalisierung oder fehlende Umlaufgitter von } \\
\text { Querungsstellen für den Radverkehr außerhalb von Knotenpunkten } \\
\text { z.B. im Zuge von Radverkehrsachsen } \\
\text { - Besonderer Bahnkörper von Straßenbahnen ist zu Lasten } \\
\text { ausreichender Flächen für andere Nutzungsansprüche } \\
\text { - Keine Verhinderung der Mitbenutzung von ÖPNV-Fahrstreifen mit } \\
\text { Gleisen durch Radfahrer }\end{array}$ \\
\hline & $\mathrm{Kfz}$ & \\
\hline & $\begin{array}{l}\text { Halte- } \\
\text { stelle }\end{array}$ & $\begin{array}{l}\text { - Freigabezeiten der Fußgängerfurten als Haltestellenzugang nicht auf } \\
\text { einfahrende ÖPNV-Fahrzeuge abgestimmt }\end{array}$ \\
\hline $\begin{array}{l}\text { Straßenbündige } \\
\text { Bahnkörper }\end{array}$ & $\begin{array}{l}\text { Fuß- } \\
\text { gänger }\end{array}$ & $\begin{array}{l}\text { - Sichteinschränkung durch abgestellte Fahrzeuge nicht zuverlässig } \\
\text { verhindert }\end{array}$ \\
\hline \multirow{3}{*}{$\begin{array}{l}\text { Unabhängige } \\
\text { Bahnkörper }\end{array}$} & $\begin{array}{l}\text { Fuß- } \\
\text { gänger }\end{array}$ & $\begin{array}{l}\text { - Fehlende Überquerungsanlage bei ausgeprägtem Überquerungsbedarf } \\
\text { - Lage der Querungshilfe außerhalb der Fußgängerüberquerungslinien } \\
\text { - das Queren wird an nicht dafür vorgesehenen Stellen nicht ausreichend } \\
\text { wirksam unterbunden }\end{array}$ \\
\hline & $\begin{array}{l}\text { Rad- } \\
\text { fahrer }\end{array}$ & $\begin{array}{l}\text { - Fehlende zusätzliche Sicherungseinrichtungen (Umlaufgitter, } \\
\text { zusätzliche Signalgeber in geringer Höhe, akustische Signale) bei } \\
\text { unübersichtlichen Querungsstellen, gradliniger Führung oder bei } \\
\text { hohem Fußgänger- und Radverkehrsaufkommen }\end{array}$ \\
\hline & $\begin{array}{l}\text { Straßen- } \\
\text { bahn }\end{array}$ & $\begin{array}{l}\text { - Fehlende Durchsetzung der zulässigen Höchstgeschwindigkeit für } \\
\text { Straßenbahnfahrzeuge } \\
\text { - geringe Sichtweiten }\end{array}$ \\
\hline
\end{tabular}




\subsection{Zusammenfassung der mikroskopischen Analyse}

Alle begangenen Örtlichkeiten der mikroskopischen Analyse waren durch erhebliche Sicherheitsdefizite der Infrastruktur gekennzeichnet, die von den Vorgaben bestehender Regelwerke abwichen.

Für die auffälligen Verkehrsanlagen in Zusammenhang mit Straßenbahnunfällen gibt es im Wesentlichen kein Erkenntnisproblem, sondern vielmehr ein Umsetzungsproblem in Form der konsequenten Anwendung und Umsetzung von Vorgaben und Hinweisen der Regelwerke.

Insbesondere wurde oftmals den allgemeinen Grundsätzen für eine sichere Gestaltung durch Erkennbarkeit, Begreifbarkeit, Einheitlichkeit, Befahrbarkeit/ Begehbarkeit und Übersichtlichkeit nicht entsprochen.

Bei den untersuchten Verkehrsanlagen, war jedoch eine Vielzahl der Unfälle auch auf mangelnde Akzeptanz der Verkehrsregelungen zurückzuführen. Daraus ist zu schließen, dass

- die Belange aller Verkehrsteilnehmer ausreichend berücksichtigt werden müssen und

- Verkehrsregelungen durch bauliche Maßnahmen zu unterstützen sind.

Es zeigte sich an den untersuchten Verkehrsanlagen, dass im Zusammenhang mit Fußgängerunfällen Querungsstellen häufig nicht an den dafür notwendigen Stellen, z.B. im Zuge von Fußgängerachsen, lagen. Auch Haltestellen in Mittellage waren häufig nur an einem Bahnsteigende mit einer Querungshilfe angebunden.

Beim Kfz-Verkehr wurde an Bahnkörpern in Mittellage häufig verbotswidrig über den Gleiskörper links abgebogen. Unfälle mit Radfahrern standen häufig im Zusammenhang mit fehlender Wahrnehmbarkeit der bevorrechtigten Straßenbahn und keiner Geschwindigkeitsdämpfung für Radfahrer an Querungsstellen.

Aus o.g. Erkenntnissen wird ersichtlich, dass Sicherheitsaudits bei Um-, Aus- und Neubau bei der Planung von Straßen und Knotenpunkten mit Straßenbahnbetrieb sowie auch bei bestehender Infrastruktur sinnvoll sind.

Charakteristische Sicherheitsdefizite konnten nach Art der Verkehrsanlage aufgestellt werden. Diese können bei Planung und Auditierung von Straßenbahnanlagen unterstützend herangezogen werden und wurden bei der Ergänzung einer Prüfliste zur Bewertung straßenbahnbezogener Infrastruktur maßgeblich mit berücksichtigt (s. Kapitel 8.4.5). 


\section{$8 \quad$ Zusammenfassung der Projektergebnisse und Empfehlungen}

\subsection{Allgemeines}

Straßenbahnunfälle sind absolut gesehen vergleichsweise selten. Auf die Beförderungs- und Verkehrsleistung bezogen ist zu konstatieren, dass Straßenbahnen ein sicheres Transportmittel sind, da sich - bezogen auf die vielen beförderten Personen - vergleichsweise wenige Unfälle mit Personenschäden ereignen. Zugleich sind Straßenbahnverkehrsinfrastrukturen vergleichsweise unsicher, da sich - bezogen auf die gefahrenen Kilometer einer Straßenbahn - viele und schwere Unfälle mit Personenschäden ereignen.

Aus den Auswertungen wurde ersichtlich, dass sich die überwiegende Anzahl der Unfälle mit Beteiligung von Straßenbahnen an Knotenpunkten ereignet. Am häufigsten handelt es sich dabei um Kollisionen mit PKWs, bei denen PKW-Fahrer Hauptverursacher sind. Diese Unfälle haben allerdings geringe Unfallfolgen. Schwere Unfälle betreffen vor allem Fußgänger und Radfahrer. Etwa $90 \%$ aller Getöteten bei Unfällen mit Straßenbahnbeteiligung sind unter Fußgängern und Radfahrern zu beklagen.

Bei den untersuchten unfallauffälligen Verkehrsanlagen, war eine Vielzahl von Unfällen auf mangelnde Akzeptanz der Verkehrsregelungen zurückzuführen. Daraus ist zu schließen, dass

- die Belange aller Verkehrsteilnehmer ausreichend berücksichtigt werden müssen und

- Verkehrsregelungen durch bauliche Maßnahmen zu unterstützen sind.

Alle begangenen Örtlichkeiten der mikroskopischen Analyse wiesen Sicherheitsdefizite der Verkehrsinfrastruktur auf, da in der Regel von den Vorgaben bestehender Regelwerke abgewichen wurde.

Für auffällige Verkehrsanlagen in Zusammenhang mit Straßenbahnunfällen gibt es im Wesentlichen kein Erkenntnisproblem, sondern handelt es sich vielmehr um ein Umsetzungsproblem in Form einer konsequenten Anwendung und Umsetzung von Vorgaben und Hinweisen der Regelwerke.

\subsection{Sicherheit nach der Art der Verkehrsbeteiligung}

\subsubsection{Fußgänger}

Unfälle zwischen Fußgängern und Straßenbahnen sind durch eine hohe Unfallschwere geprägt. Dabei sind die Fußgänger zu 79\% Hauptverursacher. Kollisionen mit Fußgängern ereignen sich überwiegend an Knotenpunkten trotz Querungshilfen, an Haltestellen und aber auch auf der „freien Strecke“.

An Strecken mit unabhängigem Bahnkörper, Bahnkörper in Seitenlage und besonderem Bahnkörper in Mittellage gibt es überproportional viele Unfälle mit Fußgängern. Häufig festgestellte Defizite bei den Ortsbesichtigungen der auffälligen Unfallstellen waren auf der „freien Strecke": fehlende gesicherte Querungsstellen im Zuge von Fußgängerachsen, Defizite in der Lichtsignalsteuerung (Phasenfolgen, lange Wartezeiten) und schlechte/geringe Wahrnehmbarkeit herannahender bevorrechtigter Straßenbahnen. 
Insofern ist diesen genannten Defiziten eine erhöhte Aufmerksamkeit zu schenken. Zur Verbesserung der Sicherheit von Fußgängern an Straßenbahnverkehrsinfrastrukturen wird Folgendes empfohlen:

- Lage und Anzahl der Querungshilfen erst nach ermitteltem Bedarf festlegen,

- Sichere linienhafte Überquerungsmöglichkeiten bei Geschäftsstraßen schaffen,

- Anordnung von Z-Übergängen,

- Lichtsignalanlagen mit Bedarfsanforderung und sehr geringen Wartezeiten für Fußgänger (wegen der hohen Akzeptanz) bevorzugen,

- Umwegefreie Haltestellenzugänge ermöglichen,

- Schaffung von Sichtfeldern zur Erhöhung der Wahrnehmbarkeit bevorrechtigter Straßenbahnfahrzeuge.

An lichtsignalgeregelten Knotenpunkten waren Fußgänger überproportional als Hauptverursacher $(+5,1 \%$ pP) beteiligt. Insofern kommt der Lichtsignalanlage hinsichtlich der Sicherheit von Fußgängern eine besondere Bedeutung zu. Häufig festgestellte Defizite bei den Ortsbesichtigungen der in der Detailanalyse untersuchten Knotenpunkte waren vor allem: lange Wartezeiten für Fußgänger, umwegebehaftete Fußgängerfurten (z.B. durch fehlende Furten in einigen Knotenpunktzufahrten) und mangelnde Wahrnehmbarkeit von Signalgebern und bevorrechtigten Straßenbahnen.

Zur Erhöhung der Fußgängersicherheit an lichtsignalgeregelten Knotenpunkten mit Straßenbahnverkehr werden daher folgende Empfehlungen gegeben:

- Schaffung von Sichtfeldern zur Erhöhung der Blickkontakte und Verbesserung der Begreifbarkeit der Bevorrechtigung von Straßenbahnfahrzeugen,

- sichere und konsequente Führung der Fußgänger zu den Furten in allen Zufahrten (z.B. durch Geländer)

- umwegefreie, gradlinige Führung über Fußgängerfurten in jedem Knotenpunktarm vorsehen, um Fehlverhalten vorzubeugen,

- Wartezeiten für Fußgänger an lichtsignalgeregelten Knotenpunkten minimieren, um die Akzeptanz zu erhöhen,

- unterschiedliche Freigabezeiten hintereinanderliegender Furten vermeiden,

\subsubsection{Radfahrer}

Radfahrer sind Hauptverursacher von etwa 9,5\% von Straßenbahnunfällen. Bei Unfällen mit schwerem Personenschaden sind Radfahrer mit einem Anteil von etwa 14,3\% überproportional als Hauptverursacher repräsentiert. Kollisionen zwischen Straßenbahn und Radfahrer ereignen sich überwiegend an Streckenabschnitten mit unabhängigem Bahnkörper und an denen mit besonderem Bahnkörper in Mittellage sowie an Knotenpunkten mit Lichtsignalanlage.

Die festgestellten Defizite sind sehr ähnlich zu denen des Fußgängerverkehrs. Deshalb sollte auf folgende Aspekte besonders geachtet werden:

- Lage und Anzahl der Querungshilfen nach entsprechendem Bedarf,

- Anordnung von Z-Übergängen mit entsprechender Dimensionierung für eine Nutzung auch für den Radverkehr 
- Getrennte Signalisierung des Radverkehrs (ggf. auch durch kurze Freigabezeiten bei minimalen Wartezeiten), insbesondere bei einer Führung im Mischverkehr ,

- Vermeidung unterschiedlicher Freigabezeiten hintereinanderliegender Radfahrerfurten

- Schaffung von Sichtfeldern zur Erhöhung der Blickkontakte und Verbesserung der Begreifbarkeit der Bevorrechtigung von Straßenbahnfahrzeugen.

\subsubsection{Kraftfahrzeuge}

Insgesamt verursachen andere Fahrzeuge des motorisierten Verkehrs (Pkw, Zweiräder etc.) durchschnittlich weniger schwere Unfälle bei Kollisionen mit Straßenbahnen im Vergleich zu schwachen Verkehrsteilnehmern (Fußgängern und Radfahrern). Etwa 60 \% der Unfälle mit leichtem Personenschaden werden durch diese Fahrzeuge verursacht. Diese Unfälle ereignen sich überwiegend an Knotenpunkten (mit oder ohne Lichtsignalanlage) und vergleichsweise seltener an Haltestellen.

Kraftfahrzeuge verursachen viele Unfälle an Knotenpunkten mit einem Bahnkörper in Mittellage. Dabei handelt es sich insbesondere um Knotenpunkte auf zweistreifigen Straßen mit besonderem Bahnkörper in Mittellage sowie auf drei- bzw. vierstreifigen Straßen mit einem straßenbündigen Bahnkörper in Mittellage.

Häufig benannte Defizite bei den Ortsbesichtigungen der auffälligen Unfallstellen standen häufig in Zusammenhang mit der Gestaltung und dem Betrieb der Lichtsignalsteuerung, der Wahrnehmbarkeit von Lichtsignalgebern und der Begreifbarkeit der Bevorrechtigung von Straßenbahnen oder vollständig fehlender Signalisierung trotz mangelnder Erkennbarkeit, Übersichtlichkeit, Begreifbarkeit und/oder falscher Berücksichtigung der relevanten Knotenstrombelastungen.

Insofern sind diesen genannten Defiziten eine erhöhte Aufmerksamkeit zu schenken und entsprechende Empfehlungen zu geben:

- technische Sicherung durch Signalisierung von Knotenpunkten jeglichen Typs ab 100 Kfz/ Tag vorsehen (auch Grundstückszufahrten),

- Wiederholung des Richtungssignals über der Fahrbahn abbiegender Kraftfahrzeuge,

- Zeit- oder Wegevorsprung für Straßenbahnen mit konfligierenden Strömen bei wiederechtlichem Befahren vorsehen, um Fehlverhalten vorzubeugen

- Schaffung von Sichtfeldern zur Erhöhung der Begreifbarkeit der Bevorrechtigung von Straßenbahnfahrzeugen und der Erkennbarkeit von Bahnübergängen an Knotenpunkten mit unabhängigem Bahnkörper oder mit einem Bahnkörper in Seitenlage.

Überdurchschnittlich häufig sind Fahrzeuge des motorisierten Verkehrs als Hauptverursacher bei Strecken mit straßenbündigen Bahnkörpern involviert. Bei der Untersuchung im Rahmen der mikroskopischen Untersuchung konnte festgestellt werden, dass eine bauliche Trennung zwischen Fahrstreifen der Kraftfahrzeuge und des Bahnkörpers auch dann häufig fehlen, wenn der Bahnkörper ausschließlich von Straßenbahnen zu befahren ist.

Insofern wird empfohlen, eigene Bahnkörper für Straßenbahnen baulich durch Borde von der Fahrbahn abzugrenzen, um das Wenden und Abbiegen an nur dafür vorgesehenen und technisch gesicherten Knotenpunkten zuzulassen. 


\subsubsection{Straßenbahnen}

Straßenbahnen verursachten etwa 15\% aller untersuchten Unfälle mit Personenschaden. Bei einem Drittel davon waren keine weiteren Verkehrsteilnehmer beteiligt. Stürze in der Straßenbahn sind mögliche Folgen nach solchen Unfällen.

Bei den vergleichsweise insgesamt wenigen Unfällen mit Getöteten ist jedoch zu konstatieren, das Straßenbahnen ca. zu 16\% Hauptverursacher der Unfälle sind. Insbesondere auf der freien Strecke unabhängiger Bahnkörper und Bahnkörper in Seitenlage steigen die Anteile als Hauptverursacher unter Beteiligung weiterer Verkehrsteilnehmer auf bis zu 1/3 der Unfälle.

Im Zusammenhang damit scheint hier eine Sensibilisierung der Straßenbahnführer für die vermeintlich sicheren Querschnitte in Hinblick z.B. auf Aufmerksamkeit und Geschwindigkeit angemessen.

Erkannte Defizite bei den Ortsbesichtigungen der auffälligen Unfallstellen waren in diesem Zusammenhang, dass in Kurven mit Sichthindernissen (Kurveninnenseite) die Anhaltesichtweite in Bezug auf die zulässige Höchstgeschwindigkeit für Straßenbahn zu prüfen ist.

\subsection{Sicherheit nach der Art der Verkehrsanlage}

\subsubsection{Knotenpunkte}

Bis zu 86 \% aller Unfälle mit Personenschaden unter der Beteiligung von Straßenbahnen ereignen sich an Knotenpunkten oder in deren Einflussbereichen.

Der überwiegende Anteil der Knotenpunktunfälle ereignet sich an lichtsignalgeregelten Knotenpunkten (58\%) und Knotenpunkten mit Vorfahrt-regelnden-Verkehrszeichen (30\%). Dies ist im Wesentlichen auf die Anzahl dieser beiden Knotenpunkttypen im Straßenbahnnetz zurückzuführen. Es konnte gezeigt werden, dass lichtsignalgeregelte Knotenpunkte bei höheren Straßenbahnverkehrsstärken eine vergleichsweise sichere Knotenpunktart ist. Vorfahrtsknoten sind per se nicht unsicher.

Bei der Betrachtung der Hauptverursacher in Abhängigkeit der Knotenpunktart war zu erkennen, dass Kraftfahrzeuge an Knotenpunkten insgesamt bei knapp der Hälfte der Straßenbahnunfälle die Hauptunfallverursacher waren. An Vorfahrtsknoten und Grundstückseinfahrten sind Kraftfahrzeuge zu über der Hälfte als Hauptverursacher an den Unfällen beteiligt. Bei Lichtsignalknoten und Kreisverkehrsplätzen ist der Anteil der Fußgänger und Radfahrer als Hauptverursacher (und auch als Verunglückte) überproportional hoch.

Insofern sind folgende Empfehlungen zu geben:

- technische Sicherung durch Signalisierung von Knotenpunkten jeglichen Typs ab $100 \mathrm{Kfz} / \mathrm{Tag}$ (auch Grundstückszufahrten z.B. von Parkplatzzufahrten),

- Wiederholung des Richtungssignals über der Fahrbahn abbiegender Kfz,

- Zeit- oder Wegevorsprung für Straßenbahnen mit konfligierenden Strömen bei widerrechtlichem Befahren,

- Zusätzliche Signalgeber für Radfahrer bei gleichzeitiger Signalisierung mit dem Kfz-Verkehr, 
- Schaffung von Sichtfeldern zur Erhöhung der Wahrnehmbarkeit bevorrechtigter Straßenbahnfahrzeuge und der Erkennbarkeit von Bahnübergängen bei Knotenpunkten unabhängiger Bahnkörper und Bahnkörpern in Seitenlage,

- Zusätzliche Sicherungseinrichtungen (akustische Signale, Signalgeber in geringer Höhe) bei unübersichtlichen Querungsstellen, gradliniger Führung, hohem Querungsbedarf an Lichtsignalknoten,

- Signalisierung mit geringen Verlustzeiten für die Fußgänger.

\subsubsection{Strecken}

Der unabhängige Bahnkörper zeigte sich insgesamt als sicherster Querschnittstyp. Auch der Bahnkörper in Seitenlage ist außerhalb der Knotenpunkte und unter Berücksichtigung aller Unfälle (BAIER/ MAIER et al. 2007b) vergleichsweise sicher. Bei Neukonzeption und Neubau von Straßenbahnnetzen und -netzteilen sollten der unabhängige Bahnkörper und der Bahnkörper in Seitenlage verstärkt in Betracht gezogen werden. Bei Neubau und grundhaften Umbauplanungen von Straßen mit Straßenbahnbetrieb sollten Bahnkörper in Seitenlage verstärkt umgesetzt werden.

Auch für derartige vergleichsweise sichere Querschnittstypen kann unter Beachtung der Verteilung von Unfallmerkmalen weiter die Verkehrssicherheit erhöht werden.

Bei Bahnkörpern in Seitenlage ist bei Entwurf und Betrieb der freien Strecke querenden Fußgängern und Radfahrern erhöhte Sorgfalt einzuräumen.

Insofern sind folgende Empfehlungen zu geben:

- Zusätzliche Sicherungseinrichtungen (akustische Signale, Signalgeber in geringer Höhe) bei unübersichtlichen Querungsstellen, gradliniger Führung, hohem Querungsbedarf.

- Schaffung von Sichtfeldern zur Erhöhung der Wahrnehmbarkeit bevorrechtigter Straßenbahnfahrzeuge.

Bei besonderen Bahnkörpern in Mittellage ist auf der freien Strecke und an Knotenpunkten insbesondere den querenden Fußgängern und Radfahrern und abbiegenden Kraftfahrzeugen an Knotenpunkten erhöhte Sorgfalt einzuräumen.

Insofern sind folgende Empfehlungen zu geben:

- Lage und Anzahl der Querungshilfen bei entsprechendem Bedarf,

- Linienhafte Querungsmöglichkeiten bei Geschäftsstraßen,

- Z-Übergänge und Signalisierung mit geringen Wartezeiten für die Fußgänger,

- Umwegefreie Haltestellenzugänge

- Zeit- oder Wegevorsprung für Straßenbahnen mit konfligierenden Strömen bei wiederechtlichem Befahren.

Bei straßenbündigen Bahnkörpern ist auf der freien Strecke und an Knotenpunkten insbesondere dem Längsverkehr und ruhendem Verkehr erhöhte Sorgfalt einzuräumen.

Insofern sind folgende Empfehlungen zu geben:

- Erkennbarkeit/ Begreifbarkeit von Haltestellen oder Knotenpunkten erhöhen,

- Frühzeitige bauliche Trennung der Richtungsfahrstreifen an Haltestellen vorsehen,

- Keine Senkrechtparkstände an Fahrstreifen mit Straßenbahnen. 
Bei unabhängigen Bahnkörpern ist auf der freien Strecke insbesondere den querenden Fußgängern und Radfahrern erhöhte Sorgfalt einzuräumen.

Insofern sind folgende Empfehlungen zu geben:

- Signalisierung und Umlaufgitter insbesondere im Zuge von Radverkehrsachsen vorsehen,

- Schaffung von Sichtfeldern zur Erhöhung der Sichtkontakte und Verbesserung der Begreifbarkeit der Bevorrechtigung von Straßenbahnfahrzeugen.

\subsection{Sicherheitshinweise zur Implementierung in Regelwerken}

\subsubsection{Fußgänger}

Wesentliche erkannte Defizite der Infrastruktur sind bereits in den Regelwerken, insbesondere der EAÖ 2013 behandelt. Bei den untersuchten auffälligen Unfallstellen blieb eine Vielzahl von Regelwerkshinweisen unberücksichtigt.

Darüber hinaus sollten folgende Punkte in den Regelwerken erwähnt oder stärker herausgearbeitet werden:

- angehobene Fahrbahnen oder Zeitinseln sollten bei Haltestellen in Mittellage und getrennten Warte- und Halteflächen die Regellösung darstellen.

- Zusätzliche akustische Signalgeber sollten an unübersichtlichen Querungsstellen, bei gradliniger Führung von Querungstellen und ggf. unter Beachtung weiterer Einsatzgrenzen häufiger zur Anwendung kommen.

- Der Rot-Nachlauf an Fußgängerquerungen der Gleiskörper sollte vermieden werden und bei Umrüstung und Neubau von Lichtsignalanlagen sollte dies grundsätzlich berücksichtigt werden.

\subsubsection{Radfahrer}

Eine Vielzahl an erkannten Defiziten der Infrastruktur für Radfahrer ergab sich insbesondere an Knotenpunkten dadurch, dass Radverkehrsanlagen hinsichtlich ihrer Begreifbarkeit, Übersichtlichkeit, Einheitlichkeit und Dimensionierung nicht den allgemeinen Entwurfsgrundsätzen von Verkehrsanlagen entsprochen haben. Die Radverkehrsführung unter beengten straßenräumlichen Verhältnissen ließ häufig Defizite erkennen.

Darüber hinaus zeigte sich an Querungsstellen eine erhöhte Gefahr für Radfahrer zu verunglücken.

Folgende Punkte sollten in den Regelwerken erwähnt oder stärker herausgearbeitet werden:

- Querungsstellen von Geh- und Radwegen, welche außerhalb des Bereiches von Straßenkreuzungen liegen und nicht technisch gesichert sind, müssen grundsätzlich zusätzlich mit Umlaufsperren oder ähnlich wirkenden Einrichtungen ausgerüstet und in der Regel durch Lichtzeichen signalisiert sein.

- Bei der Führung des Radverkehrs gemeinsam auf oder parallel zur Fahrbahn werden die Sicherheitseinrichtungen der Straße im Regelfall jedoch ergänzt durch Signalgeber für den Radverkehr genutzt. 
- An Rampen von Hochbahnsteigen sollten Radfahrer durch Umlaufgitter bzw. Poller vor schnellem Herabrollen und anschließendem zügigen Queren der Gleise geschützt werden.

- Die Prinzipskizze einer Straßenbahnhaltestelle in Mittellage mit Seitenbahnsteigen sieht nach [EAÖ 2013] keine gradlinige Führung des querenden Fußgängerverkehrs (und i.A. auch Radverkehrs) vor. Dagegen wird nach Prinzipskizze einer Straßenbahnhaltestelle in Mittellage mit Mittelbahnsteig nach [EAÖ 2013] die querenden Fußgänger gradlinig über die Haltestelleninsel geführt:

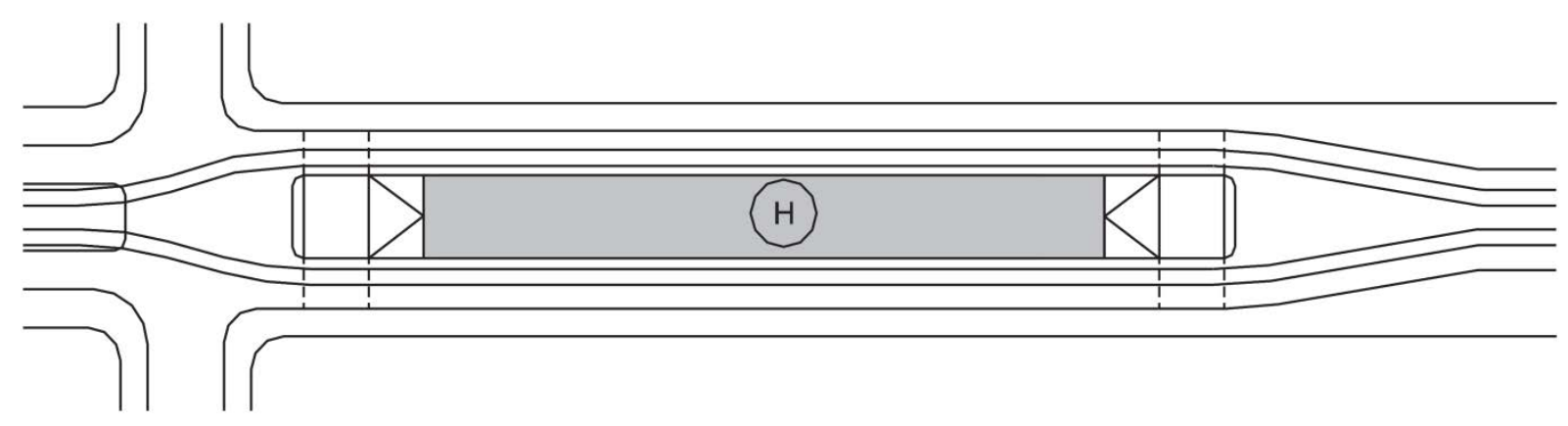

Abbildung 8-35: Prinzipskizze Haltestelle in Insellage mit Mittelbahnsteig Quelle: [EAÖ 2013], S. 72

Als Alternative wäre hinsichtlich der Verkehrssicherheit zu prüfen, ob nicht ein Versatz der gegenüberliegenden Furten die Blickbeziehungen auf die Straßenbahnfahrzeuge erhöht und die Geschwindigkeit querender Radfahrer reduziert.

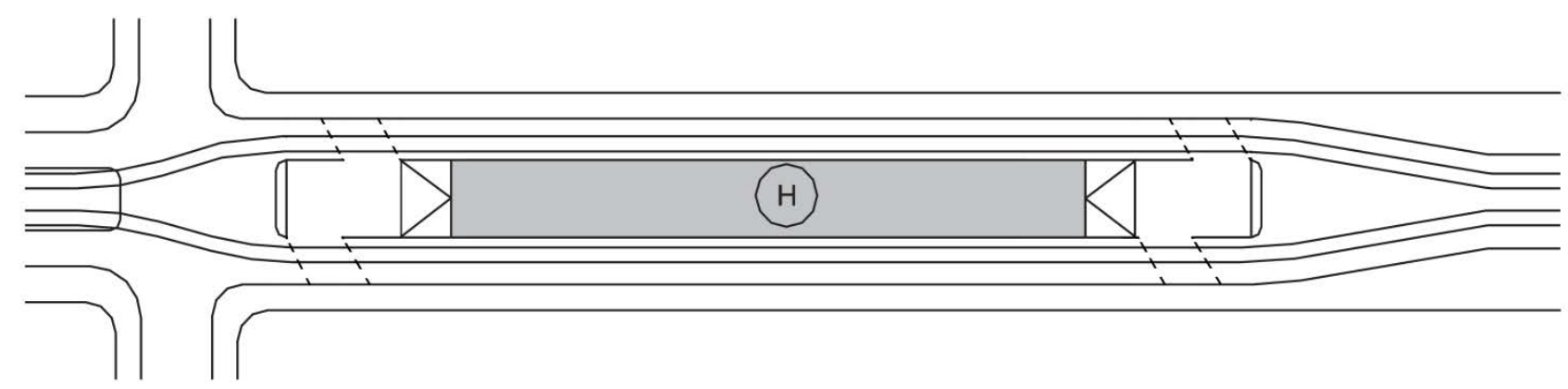

Abbildung 8-36: Alternativlösung Prinzipskizze Haltestelle in Insellage mit Mittelbahnsteig Quelle: eigene Darstellung in Anlehnung an [EAÖ 2013], S. 72

\subsubsection{Kraftfahrzeuge}

Wesentliche erkannte Defizite der Infrastruktur sind bereits in den Regelwerken behandelt. Bei den untersuchten auffälligen Unfallstellen blieb eine Vielzahl von Regelwerkshinweisen unberücksichtigt. Eine Konkretisierung und Verschärfung der Regelwerksvorgaben wären, insbesondere hinsichtlich der Begreifbarkeit der Signalsteuerung sinnvoll.

Darüber hinaus sollten folgende Punkte in den Regelwerken erwähnt oder stärker herausgearbeitet werden:

- Querungen von Straßen, Wegen und Plätzen mit jeglicher Führung von Straßenbahnen müssen gegenüber dem Kfz-Verkehr technisch gesichert sein. Dies gilt nicht für Querungen, die innerhalb eines Tages in der Regel von nicht mehr als 100 Kraftfahrzeugen überquert werden und die durch die Übersicht auf die Bahnstrecke gesichert sind. 
- Wird über ein straßenbegleitenden unabhängigen Bahnkörper auch einstreifig abgebogen,dann muss das Richtungssignal über der Fahrbahn wiederholt werden.

- Nicht zulässige Fahrbeziehungen mit konfligierenden Strömen bei widerechtlichem Befahren an Lichtsignalknotenpunkten sollten durch das Signalprogramm ausgeschlossen werden. Ist dies nicht möglich, sollte zur Erhöhung der Erkennbarkeit der Straßenbahn ein Zeit- oder Wegvorsprung gegeben werden.

- Zur Erhöhung der Erkennbarkeit sollte auch bei lichtsignalgeregelten Knotenpunkten gegenüber der Straßenbahn die Annäherungssichtweite gegeben sein.

\subsubsection{Straßenbahnen}

Bei Unfällen mit Straßenbahnen ist die Straßenbahn selbst nur in geringen Fällen Hauptverursacher des Unfalls. Darüber sind die Personenschäden bei Unfällen mit Hauptverursacher Straßenbahn häufig auf Verletzungen bei Passagieren der Straßenbahn zurückzuführen, ohne das weitere Verkehrsteilnehmer zu Schaden gekommen sind. Stürze in der Bahn durch beispielsweise Bremsungen könnten eine mögliche Ursache sein.

Darüber hinaus sollten folgende Punkte in den Regelwerken erwähnt oder stärker herausgearbeitet werden:

- Eine eindeutige Signalisierung an signalisierten Querungsstellen z.B. in Verbindung mit den Signalbildern F0 (Halt) und F1 (Fahrt freigegeben) ist in jedem Fall herzustellen.

- Das Fahren auf Sicht sollte durch tabellierte Sichtweiten (und deren Überprüfung) in Abhängigkeit der zulässigen Höchstgeschwindigkeit sichergestellt sein. 


\subsection{5 ergänzende Sicherheitshinweise in der ESAS-Defizitcheckliste}

Die Empfehlungen für das Sicherheitsaudit von Straßen (ESAS 2002) beschreiben ein formalisiertes, einheitliches Verfahren bei Planungen von Verkehrsanlagen Sicherheitsdefizite vor Realisierung durch geschulte und erfahrene Auditoren erkennen zu können und nach Abwägung anderen planerischen Belangen nach Möglichkeit zu beseitigen. Im Zusammenhang mit dem Erkennen der Sicherheitsdefizite kann das Verfahren auch auf Verkehrsanlagen im Bestand ausgeweitet werden.

Als Unterstützung und für die Selbstkontrolle des Auditors sind den ESAS Checklisten beigefügt, die typische Defizite von Verkehrsanlagen aufzeigen. Aufgrund der Übersichtlichkeit und Handhabbarkeit können die Checklisten nur bis zu einem gewissen Detailierungsgrad und Umfang in der ESAS enthalten sein. Es ist jedoch vorstellbar, dass für spezielle Fragestellungen, Themen und Anwender einzelne Checklisten detaillierter ausgearbeitet werden sollten. Für die Defizite mit Konfliktpotentialen für Straßenbahnunfälle sollte dies umgesetzt werden. Im Anhang A.9 ist eine erweiterte Defizitcheckliste mit speziellem Bezug zur Straßenbahn enthalten.

Die Defizitcheckliste stellt eine Ergänzung der durch BAIER ${ }^{109}$ im Rahmen der Fortschreibung der ESAS erstellten Prüfpunkte für Innerortsstraßen dar. Die grau hinterlegten Checkpunkte mit Bezug zu Straßenbahnverkehr sind daraus übernommen. Die Checkpunkte in schwarzer Schrift stellen Vorschläge für eine sinnvolle Erweiterung dar. 
Mit dem Forschungsvorhaben "Maßnahmen zur Reduzierung von Unfällen mit Straßenbahnen" konnten neue Erkenntnisse über die Struktur der Unfälle mit Straßenbahnbeteiligung und zur Verbesserung von der Verkehrssicherheit der Straßenbahnverkehrsinfrastruktur gewonnen werden.

Zur Bearbeitung des Forschungsvorhabens wurden im Wesentlichen zwei methodische Ansätze kombiniert.

In einem ersten Schritt erfolgte für 58 deutsche Städte mit Straßenbahnbetrieb eine statistische Auswertung (makroskopische Analyse) des Unfallgeschehens. Es wurden ausschließlich Unfälle mit Personenschaden unter Beteiligung von Straßenbahnen betrachtet. Dazu erfolgte eine Abfrage der Unfalldaten der Jahre 2009, 2010 und 2011 bei den zuständigen Behörden. Ergänzt wurden diese Unfalldaten um straßenräumliche typisierte Merkmale, sodass die statistische Auswertung auf diese Typisierungsmerkmale ausgeweitet werden konnte.

Der zweite Schritt des Forschungsvorhabens bestand aus einer vertiefenden Untersuchung von Knotenpunkten und Streckenabschnitten mit auffälligem Unfallgeschehen (mikroskopische Analyse). Ziel dabei war es, Sicherheitsdefizite zu erfassen, die auf eine unsichere Gestaltung der Verkehrsanlagen zurückzuführen sind.

Ergänzt wurden diese beiden methodischen Ansätze um weitere grundlegende aufeinander aufbauende Arbeitspakete und daraus abgeleitete Empfehlungen.

Eine umfassende Literaturanalyse ergab, dass der Schwerpunkt bisheriger Forschungsvorhaben insbesondere auf Straßenbahnhaltestellen lag. Häufig auf Basis von Konfliktbeobachtungen, wurden dafür bereits weitreichende Erkenntnisse gewonnen:

- Annährungsbereiche an Haltestellen weisen hohe Gefährdungspotenziale für Fußgänger auf.

- Haltestellen am Fahrbahnrand sind insgesamt sicherer als Haltestellen in Mittellage.

- Die Führung des Radverkehrs ist insbesondere bei Haltestellen in Seitanlage kritisch.

- Fußgänger haben eine hohe Umwegeempfindlichkeit.

- „Laufeinsteiger", die noch eine bereits einfahrende oder schon haltende Straßenbahn erreichen wollen, sind die gefährdetste Gruppe.

- Das Unfallgeschehen im motorisierten Verkehr ist maßgeblich durch Unfälle im Längsverkehr geprägt.

- Zeitinseln ergeben nicht zwangsläufig günstigere Unfallraten als bei unsignalisierten Haltestellen in Mittellage mit Wartefläche am Fahrbahnrand.

Aus den genannten Erkenntnissen ergeben sich mit den untersuchten Literaturquellen Maßnahmen zur Verbesserung der Verkehrssicherheit bzgl. der Haltestellenform, Erkennbarkeit von Haltestellen, Schaffung sicherer Querungshilfen und Radverkehrsführungen sowie der Anpassung von Kfz-Geschwindigkeiten und der Ordnung des ruhenden Verkehrs an Haltestellen. 
Für Strecken liegt eine Untersuchung, von BAIER/ MAIER ${ }^{110}$ vor, bei der für verschiedene Querschnittstypen eine Vergleichsuntersuchung bzgl. des Sicherheitsgrades von Stadtstraßen mit und ohne schienengebundenen ÖPNV durchgeführt wurde und Unfallkostenraten und -dichten ermittelt wurden. Demnach sind bezogen auf das Gesamtunfallgeschehen Straßen mit besonderem Bahnkörper in Seitenlage am sichersten, gefolgt von besonderen Bahnkörpern in Mittellage und von straßenbündigen Bahnkörpern. Zudem konnte festgestellt werden, dass besondere Bahnkörper bzgl. querender Fußgänger kritisch sind. Straßenbündige Bahnkörper weißen dagegen bzgl. des Kfz-Verkehrs ein erhöhtes Unfallrisiko auf.

Obwohl sich über $80 \%$ der Straßenbahnunfälle an Knotenpunkten ereignen, lagen dafür bislang keine belastbaren Untersuchungen vor.

Unabhängig davon werden in den aktuellen Regelwerken umfangreiche Aussagen zur Verkehrssicherheit von Straßenbahninfrastrukturen getroffen. Insbesondere in den Empfehlungen für die Anlagen des öffentlichen Personennahverkehrs (EAÖ 2013), den Richtlinien für die Anlage von Stadtstraßen (RASt 2006) und den Empfehlungen für Radverkehrsanlagen (ERA 2010) werden weitreichende sicherheitsrelevante verkehrsplanerische Vorgaben für die Gestaltung und Vorgaben an betriebliche Anforderungen der Straßenbahninfrastruktur gegeben.

Im Rahmen einer vergleichenden Beurteilung der Verkehrssicherheit wurden Unfallzahlen und Unfallschwere von Personenkraftwagen (PKW), Bus und Straßenbahn gegenüber gestellt. Dies erfolgte anhand der Unfallkosten, die sich aus den volkswirtschaftlichen Kosten durch die Straßenverkehrsunfälle ergeben.

Darüber hinaus wurden aussagekräftige Unfallkenngrößen in Abhängigkeit der Fahrleistung und in Abhängigkeit der Beförderungsleistung miteinander verglichen.

Bei der Betrachtung der absoluten Unfallzahlen war zu erkennen, dass Unfälle mit Beteiligung von Straßenbahnen vergleichsweise seltener sind. Auch die Zahl der Schwer- und Leichtverletzen ist absolut betrachtet bei Straßenbahnen im Vergleich gering. Bei Betrachtung der Zahl der Getöteten war allerdings zu erkennen, dass diese bei Straßenbahnen höher ist, als bei Bussen.

Die Unfallschwere bei Straßenbahnunfällen ist insgesamt deutlich höher als bei Unfällen mit PKW und Bussen. Als Maß der Unfallschwere waren die durchschnittlichen Kosten je Unfall innerorts gegenüberzustellen. Bei der Betrachtung der Unfälle mit Personenschaden zeigte sich für Straßenbahnunfälle eine um ca. 50\% höhere Unfallschwere gegenüber PKW und ca. $30 \%$ höhere Unfallschwere gegenüber Unfällen mit Bussen.

Der relative Bezug auf die Fahrleistung berücksichtigt, dass Straßenbahnen im Vergleich, insbesondere zum PKW, aber auch zum Bus eine wesentlich geringere Fahrleistung aufweisen. Die damit verbundene geringere Unfallwahrscheinlichkeit wird somit in der Bewertung durch die Berechnung der Unfallrate und die Folgen dieser Unfälle durch die Unfallkostenrate erfasst.

Die Betrachtung der Beförderungsleistung berücksichtigt, dass Fahrten von Straßenbahnen nur durch eine Vielzahl von zusätzlichen Fahrten durch Busse und noch mehr durch PKW kompensiert werden könnte.

Es zeigte sich, dass bezogen auf die gefahrenen Kilometer die Straßenbahn ein vergleichsweise unsicheres Verkehrsmittel ist. Es war zu erkennen, dass die Unfallkostenrate bei Straßenbahnen

${ }^{110}$ [BAIER, Maier et al. 2007b] 
im Vergleich zum PKW um das 8-fache höher liegt. Bezogen auf die beförderten Personen ist die Straßenbahn jedoch ein vergleichsweise sicheres Transportmittel. Das Unfallkostenrisiko (Unfallkosten je 1.000 Personen-Kilometer) bei Straßenbahnen beträgt etwa nur $20 \%$ von dem der PKW.

Um längenbezogene Merkmalsverteilungen und differenzierte Unfallkenngrößen zu erhalten, wurde für fünf Städte (Dresden, Bremen, Köln, Karlsruhe und Erfurt ) eine gesonderte Netzanalyse bezüglich der Merkmalsausprägungen differenziert nach Querschnittstypen und RASt-

Entwurfssituationen durchgeführt.

Basis der Unfalldaten bildeten die vorliegenden georeferenzierten, polizeilich erfassten Unfalldaten, die in ein Geoinformationssystem (GIS) importiert und auf das jeweilige Liniennetz übertragen wurden.

Die Unfälle wurden dabei Netzsegmenten zugeteilt. Netzsegmente mit derselben Merkmalsausprägung wurden zusammengefasst und die dazugehörigen Unfallkenngrößen berechnet und analysiert.

Es zeigte sich, dass der 3- bzw. 4-streifige Querschnitt mit besonderem Bahnkörper in Mittellage hinsichtlich der Unfallfolgen besonders auffällig ist, selbst dann, wenn nur die Unfälle außerhalb von Knotenpunkten betrachtet werden. Die makroskopische Unfallanalyse weist zudem hier eine besonders hohe Unfallschwere auf.

Der unabhängige Bahnkörper zeigte sich insgesamt als sicherster Querschnittstyp, auch wenn die Unfälle, die hier geschehen besonders schwer sind. Dieser Querschnitt ist jedoch in der Regel eher bei Neubau oder am Stadtrand zu realisieren.

Der Querschnitt mit Führung der Straßenbahn in Seitenlage zeigte unter Berücksichtigung der Knotenpunktunfälle keinen wesentlichen Sicherheitsgewinn. Außerhalb der Knotenpunkte ist dieser Querschnitt allerdings ähnlich sicher wie der unabhängige Bahnkörper, auch wenn die Unfälle, die hier geschehen, besonders schwer sind.

Unter Berücksichtigung der Untersuchungen von BAIER/ MAIER, bei der auch Unfälle des KfzVerkehrs betrachtet wurden, können der unabhängige Bahnkörper und der Bahnkörper in Seitenlage als die insgesamt sichersten Führungsformen von Straßenbahnen bewertet werden.

Die Auswertung von Unfalldaten und straßenräumlichen Daten wurde in der makroskopischen Analyse vertieft. Für die Auswertung lagen die Unfalldaten der polizeilich aufgenommenen Unfälle mit Personenschaden der Jahre 2009 bis 2011 mit Beteiligung von Straßenbahnen von 58 deutschen Städten vor. Eine nahezu vollständige Erfassung aller Straßenbahnunfälle Deutschlands über diese drei Jahre war damit gegeben.

Für Auswertungen nach fahrleistungsbezogenen Kenngrößen von Straßenbahnunfällen wurden diese in Bezug zur Straßenbahnverkehrsstärke gesetzt, die querschnittsbezogen aus den Fahrplandaten ermittelt wurde.

Neben den polizeilich erfassten Merkmalen der vorliegenden Unfalldaten wurden den lokalisierten Unfällen weitere verkehrliche und straßenräumliche Merkmale für die anschließende Bewertung der Straßenverkehrsinfrastruktur zugeordnet. Die Erfassung erfolgte im Wesentlichen aus 
Luftbildern und Befahrungsbildern bekannter Kartendienste. Die zusätzlich erhobenen Informationen sind Merkmale z.B. zur RASt-Entwurfssituation, Querschnittstyp, Haltestellenform, Knotenpunktmerkmale (Abstand, Knotenpunktform, Anzahl Zufahrten und Fahrstreifen, Querschnittstypen der Zufahrten) und Querungshilfenmerkmale (Abstand, Art der Querungshilfe).

Die Ergebnisse der Analyse der über 4.000 Unfälle mit Personenschaden unter Beteiligung von Straßenbahnen wurden in Diagrammen mit zugehörigen Kurzbeschreibungen und Kurzinterpretationen dokumentiert und wesentliche Erkenntnisse zusammengefasst:

Fußgänger haben bei den Getöteten und bei den Schwerverletzten den weitaus größten Anteil. Radfahrer sind bei Unfällen mit schwerer Unfallfolge ebenfalls überproportional betroffen. Bei den Leichtverletzten dominieren dagegen die Insassen von Straßenbahnen und PKW.

Bei besonderer Betrachtung der Getöteten und Schwerverletzten nach der Lage der Unfallstelle im Netz zeigt sich, dass Fußgänger überproportional an Strecken und Haltestellen schwer verunglücken. Absolut ereignen sich die meisten Unfälle mit Schweren Personenschaden auch bei Fußgängern jedoch an den Knotenpunkten.

Bei Betrachtung der Hauptverursacher aller Straßenbahnunfälle mit Personenschaden war zu erkennen, dass Straßenbahnen in den wenigsten Fällen (15,7\%) selbst Hauptverursacher von Straßenbahnunfällen sind; davon sind ca. ein Drittel Alleinunfälle.

Insbesondere 3- bzw. 4-streifige Straßen mit Bahnkörpern in Mittellage und lichtsignalgeregelte Knotenpunkte weisen eine hohe Unfallschwere mit jeweils hohen Anteilen an Fußgängern als Hauptverursacher auf.

Lichtsignalgeregelte Knotenpunkte sind bei höheren Straßenbahnverkehrsstärken eine vergleichsweise sichere Knotenpunktart. Vorfahrtsknoten sind nicht immer unsicher. Ungesicherte Straßenquerungen durch die Straßenbahnen scheinen tendenziell sicherer zu sein. Dies lässt vermuten, vor allem wegen der hier herrschenden Vorfahrtsregelung „Rechts-vor-Links“, dass sich die Straßenfahrer (aber auch die übrigen Verkehrsteilnehmer) besonders aufmerksam verhalten und im Annäherungsbereich derartiger Querungen ihre Geschwindigkeit vorher deutlich reduzieren und somit von den übrigen Verkehrsteilnehmern rechtzeitig wahrgenommen werden. Bei diesen Kontenpunktarten zeigt eine zwei- bis dreifache hohe Unfallkostenrate bei niedrigeren Straßenbahnverkehrsstärken.

Aufgrund der geringen Fallzahlen ist für Kreisverkehrsplätze eine Aussage hinsichtlich der Verkehrssicherheit kaum möglich. Es konnte jedoch festgestellt werden, dass die Unfallschwere insbesondere an signalisierten Kreisverkehrsplätzen vergleichsweise gering ist. Aufgrund der Unfallanzahl und der vergleichsweise geringen Straßenbahnverkehrsstärken der untersuchten Kreisverkehrsplätze ist die Unfallkostenrate dieser Knotenpunktarten jedoch vergleichsweise hoch.

Besondere Bahnkörper in Mittellage bei drei oder vier Fahrstreifen, Bahnkörper in Seitenlage und unabhängige Bahnkörper weisen die schwersten Unfälle auf.

Um Rückschlüsse auf mögliche unfallbegünstigende Umstände der einzelnen Verkehrsanlagen zu erhalten, wurde eine Detailanalyse einschließlich einer Ortsbesichtigung an ausgewählten 21 Knotenpunkten und 11 Streckenabschnitten durchgeführt. 
Ziel war es, weitere straßenräumliche Merkmale und Besonderheiten aufzunehmen sowie Defizite der Infrastruktur zu erkennen. Dafür wurden Bestandteile der Örtlichen Unfalluntersuchung nach M UKo und des Sicherheitsaudits nach ESAS zusammengeführt.

Die Dokumentation und Darstellung der Erkenntnisse der Ortsbesichtigungen erfolgte in Steckbriefen.

Im Ergebnis zeigten sich an allen begangenen Örtlichkeiten Abweichungen von den Vorgaben der aktuellen Regelwerke, die sicherheitsrelevant sein können.

Insbesondere wurde oftmals den allgemeinen Grundsätzen für eine sichere Gestaltung durch Erkennbarkeit, Begreifbarkeit, Einheitlichkeit, Befahrbarkeit/ Begehbarkeit und Übersichtlichkeit nicht entsprochen.

Bei den untersuchten Verkehrsanlagen, war jedoch eine Vielzahl der Unfälle auch auf mangelnde Akzeptanz der Verkehrsregelungen zurückzuführen.

Es zeigte sich an den untersuchten Verkehrsanlagen, dass im Zusammenhang mit Fußgängerunfällen Querungsstellen häufig nicht an den dafür notwendigen Stellen, z.B. im Zuge von Fußgängerachsen, lagen. Auch Haltestellen in Mittellage waren häufig nur an einem Bahnsteigende mit einer Querungshilfe angebunden.

Beim Kfz-Verkehr wurde an Bahnkörpern in Mittellage häufig verbotswidrig über den Gleiskörper links abgebogen. Unfälle mit Radfahrern standen häufig im Zusammenhang mit fehlender Wahrnehmbarkeit der bevorrechtigten Straßenbahn und keiner Geschwindigkeitsdämpfung für Radfahrer an Querungsstellen. Darüber hinaus konnten weitere charakteristische Sicherheitsdefiziten an Knotenpunkten und Strecken ermittelt. Darunter zählen unter anderem:

- schlechte Erkennbarkeit, Begreifbarkeit und Übersichtlichkeit von Knotenpunkten,

- unterschiedliche Freigabezeiten hintereinanderliegender Fußgängerfurten,

- eingeschränkte Sicht auf die Signalgeber,

- fehlende Überquerungsanlagen für Radfahrer über bevorrechtigte Zufahrten,

- Nachtabschaltung von Signalanlagen.

Aus o.g. Erkenntnissen wird ersichtlich, dass Sicherheitsaudits bei Um-, Aus- und Neubau bei der Planung von Straßen und Knotenpunkten mit Straßenbahnbetrieb sowie auch bei bestehender Infrastruktur sinnvoll sind.

Die Gesamtheit der ermittelten charakteristische Sicherheitsdefiziten wurden nach Art der Verkehrsanlage differenziert. Diese können bei Planung und Auditierung von Straßenbahnanlagen unterstützend herangezogen werden. Aus den Ergebnissen wurde eine die ESAS ergänzende Prüfliste zur Bewertung straßenbahnbezogener Infrastruktur erstellt.

Aus den gewonnenen Erkenntnissen lassen sich insgesamt folgende Empfehlungen ableiten:

- Bei Neu-/Um- und Ausbau sind mehrstreifige Querschnitte mit besonderem Bahnkörper in Mittellage zu vermeiden. Bevorzugt sollte eine Führung in Seitenlage oder auf unabhängigen Bahnkörpern realisiert werden. Auf ausreichende und gesicherte Überquerungsstellen für Fußgänger und auf gesicherte Führung abbiegender Kraftfahrzeuge an Knotenpunkten ist besonders zu achten. 
- An bestehenden Querschnitten sind gesicherte Fußgängerquerungen nachzurüsten. Ungesichertes Wenden und Abbiegen ist baulich bzw. verkehrstechnisch zu unterbinden.

- Straßenbahnen sollten an signalisierten Knotenpunkten gesonderte Phasen erhalten (konfliktfreie Führung).

- Verbindliche Sicherheitsaudits bei Planung und für den Bestand sollten eingeführt werden, um Sicherheitsdefizite zu vermeiden bzw. zu reduzieren. Hierfür wurde eine Checkliste entwickelt.

- Die Entwicklung fahrzeugseitiger Technologien zur schnelleren Erkennung von Fußgängern und zur Erhöhung ihrer Aufmerksamkeit (Automatisches Warnsignal) soll vorangetrieben werden. Derartige Systeme sollten sinnvollerweise mit einer Bremsunterstützung für den Straßenbahnfahrer kombiniert werden.

- Geeignete Kampagnen zur Sensibilisierung aller Verkehrsteilnehmer sollten entwickelt werden. Dies gilt vor allem für die Straßenbahnnutzer in Hinsicht auf die Besonderheiten und Gefahrenpotenziale von Straßenbahnen.

Aus der Netzanalyse und den Erkenntnissen der mikroskopischen Analyse sind darüber hinaus für die differenzierten Querschnitte folgende detaillierte Empfehlungen für eine Verbesserung der Verkehrssicherheit einzelner Verkehrsteilnehmergruppen zu treffen:

\section{Bahnkörper in Seitenlage}

An Knotenpunkten wird eine Verbesserung der Verkehrssicherheit bzgl. abbiegender, einbiegender und kreuzender Kraftfahrzeuge und Radfahrer, u.a. durch folgende Maßnahmen geschaffen:

- Signalisierung von Knotenpunkten ab 100 Kfz/ Tag (auch Grundstückszufahrten),

- Wiederholung des Richtungssignals über der Fahrbahn abbiegender Kfz,

- Zeit- oder Wegevorsprung für Straßenbahnen mit konfligierenden Strömen bei widerrechtlichem Befahren,

- Zusätzliche Signalgeber für Radfahrer bei gleichzeitiger Signalisierung mit dem Kfz-Verkehr.

Auf der freien Strecke ergeben sich Sicherheitsverbesserungen bzgl. querender Fußgänger und Radfahrer, u.a. durch:

- Zusätzliche Sicherungseinrichtungen (akustische Signale, Signalgeber in geringer Höhe) bei hohem Querungsbedarf, vor allem an unübersichtlichen Stellen,

- Schaffung von Sichtfeldern zur Erhöhung der Wahrnehmbarkeit bevorrechtigter Straßenbahnfahrzeuge.

\section{Besondere Bahnkörper in Mittellage}

An Knotenpunkten und der freien Strecke sind bzgl. der querenden Fußgänger, Radfahrer und abbiegender Kraftfahrzeuge, Sicherheitsgewinne u.a. durch folgende Maßnahmen zu erwarten:

- Lage und Anzahl der Querungshilfen nach entsprechendem Bedarf,

- Linienhafte Querungsmöglichkeiten bei Geschäftsstraßen,

○ Z-Übergänge und Signalisierung mit geringen Verlustzeiten für die Fußgänger,

- Umwegefreie Haltestellenzugänge, 
- Zeit- oder Wegevorsprung für Straßenbahnen mit konfligierenden Strömen bei widerrechtlichem Befahren.

\section{Straßenbündige Bahnkörper}

An Knotenpunkten und der freien Strecke lassen insbesondere für den Längsverkehr und ruhendem Verkehr folgende Maßnahmen Sicherheitsverbesserungen erwarten:

- Erkennbarkeit/ Begreifbarkeit von Haltestellen oder Knotenpunkten,

- Frühzeitige bauliche Trennung der Richtungsfahrstreifen an Haltestellen,

- Keine Senkrechtparkstände an Fahrstreifen mit Straßenbahnen.

\section{Unabhängige Bahnkörper}

An Knotenpunkten wird eine Verbesserung der Verkehrssicherheit bzgl. der kreuzender Kraftfahrzeuge, Radfahrer und querender Fußgänger, u.a. durch folgende Maßnahmen geschaffen:

- Erkennbarkeit von Bahnübergängen,

- Schaffung von Sichtfeldern zur Erhöhung der Wahrnehmbarkeit bevorrechtigter Straßenbahnfahrzeuge.

Auf der freien Strecke ergeben sich Sicherheitsverbesserungen insbesondere bzgl. den querenden Fußgängern und Radfahrern u.a. durch:

- Signalisierung und Umlaufgitter insbesondere im Zuge von Radverkehrsachsen,

- Schaffung von Sichtfeldern zur Erhöhung der Wahrnehmbarkeit bevorrechtigter Straßenbahnfahrzeuge. 


\section{Literaturverzeichnis}

AEG 2013. Bundesrepublik Deutschland,

Allgemeines Eisenbahngesetz

Ausfertigungsdatum: 27.12.1993 / zuletzt geändert: 07.08.2013

Aerowest GmbH,

Geodatenserver Aerowest $\mathrm{GmbH}$

Flughafenring 11, 44319 Dortmund

www.aerowest.de

aufgerufen zwischen 01.05 .2015 bis 15.06 .2015

ArcGIS, ArcMap Version 10.0, ESRI Inc.

Environmental Systems Research Institute (ESRI)

Redlands (Kalifornien, USA)

BAIER 2014. Baier, R.

Checklisten zum Sicherheitsaudit, Defizitarten innerorts

Entwurf 14.10.2014 (Verschickung durch FGSV an Mitglieder des AA 2.7)

unveröffentlicht

BAIER, MAIER et al. 2007a. Baier, R., Maier R., Benthaus, D., Klemps, A., Enke, M., Schüller, H. Potenziale zur Verringerung des Unfallgeschehens an Haltestellen des ÖPNV/ÖPSV im Auftrag von: Bundesanstalt für Straßenwesen

Berichte der Bundesanstalt für Straßenwesen, M 190

Bericht zum Forschungsprojekt FE 82.276/2004

Verlag für neue Wissenschaft GmbH, Bremerhaven / ISBN: 978-3-86509-752-1

Bergisch Gladbach, Bremerhaven 2007

BAIER, MAIER et al. 2007b. Baier, R., Maier R., Aurich, A., Klemps, A.

Sicherheitsgrad von Stadtstraßen mit und ohne schienengebundenem ÖPNV

im Auftrag von: Unfallforschung der Versicherer

Schlussbericht, 30.06.2007

unveröffentlicht

BASt 2011. Bundesanstalt für Straßenwesen; Martina Straube

Volkswirtschaftliche Kosten durch Straßenverkehrsunfälle 2009

Forschung kompakt 04/11

Bergisch Gladbach 2011

BING MAPS 2014. Microsoft Corporation

https://www.bing.com/maps/

aufgerufen zwischen 01.06.2014 bis 07.11.2014

BOStrab 2007. Bundesrepublik Deutschland,

Verordnung über den Bau und Betrieb der Straßenbahnen (Straßenbahn-Bau- und

Betriebsordnung - BOStrab)

Ausfertigungsdatum: 11.12.1987 / zuletzt geändert: 08.11.2007 
DESTATIS 2010. Statistisches Bundesamt, Verkehrsunfälle 2009

Fachserie 8, Reihe 7, 2009.

Wiesbaden 2010

DESTATIS 2011. Statistisches Bundesamt, Verkehrsunfälle

Fachserie 8, Reihe 7, 2010.

Wiesbaden 2011

DESTATIS 2012. Statistisches Bundesamt,

Verkehrsunfälle

Fachserie 8, Reihe 7, 2011.

Wiesbaden 2012

DESTATIS 2013a. Statistisches Bundesamt,

Schienenverkehrsunfallstatistik 2013

https://www.destatis.de/DE/ZahlenFakten/Wirtschaftsbereiche/TransportVerkehr/Verkehrsunfaelle/

Tabellen/Strassenbahnunfaelle.html

aufgerufen am 12.03.2014, 11:22 MESZ

Wiesbaden 2014

DESTATIS 2013b. Statistisches Bundesamt,

Qualitätsbericht, Statistik der Straßenverkehrsunfälle

Wiesbaden 29.08.2013

DESTATIS 2013c. Statistisches Bundesamt,

Qualitätsbericht, Schienenverkehrsunfallstatistik

Wiesbaden 29.08.2013

DESTATIS 2014. Statistisches Bundesamt,

Verkehrsunfälle

Fachserie 8, Reihe 7, 2013

Wiesbaden 2014

DITTEMER 1990. Dittemer, T.

ÖPNV-Haltestellen mit Kap: Verkehrsablauf und Verkehrssicherheit untersucht an

Straßenbahnhaltestellen

Grüne Reihe, Heft 14

Fachgebiet Verkehrswesen, Universität Kaiserslautern

Kaiserslautern 1990

EAHV 1993. Forschungsgesellschaft für Straßen- und Verkehrswesen e. V. (FGSV), Empfehlungen für die Anlage von Hauptverkehrsstraßen

FGSV-Nr.: 286 / ISBN: 978-3-78121-352-4

Ausgabe 1993 / Fassung 1993

Köln 1993

EAÖ 2003. Forschungsgesellschaft für Straßen- und Verkehrswesen e. V. (FGSV), Empfehlungen für die Anlagen des öffentlichen Personennahverkehrs 
FGSV-Nr.: 289 / ISBN: -

Ausgabe 2003 / Fassung 2003

Köln 2003

EAÖ 2013. Forschungsgesellschaft für Straßen- und Verkehrswesen e. V. (FGSV), Empfehlungen für die Anlagen des öffentlichen Personennahverkehrs

FGSV-Nr.: 289 / ISBN: 978-3-86446-054-8

Ausgabe 2013 / Fassung 2013

Köln 2013

EAR 2005. Forschungsgesellschaft für Straßen- und Verkehrswesen e. V. (FGSV), Empfehlungen für Anlagen des ruhenden Verkehrs

FGSV-Nr.: 283 / ISBN: 978-3-78121-632-7

Ausgabe 2005 / Fassung 2005

Köln 2005

EBO 2012. Bundesrepublik Deutschland,

Eisenbahn-Bau- und Betriebsordnung

Ausfertigungsdatum: 08.05.1967 / zuletzt geändert: 25.07.2012

EFA 2002. Forschungsgesellschaft für Straßen- und Verkehrswesen e. V. (FGSV), Empfehlungen für Fußgängerverkehrsanlagen

FGSV-Nr.: 288 / ISBN: -

Ausgabe 2002 / Fassung 2002

Köln 2002

ERA 2010. Forschungsgesellschaft für Straßen- und Verkehrswesen e. V. (FGSV), Empfehlungen für Radverkehrsanlagen

FGSV-Nr.: 284 / ISBN: 78-3-941790-63-6

Ausgabe 2010 / Fassung 2010

Köln 2010

ESAS 2002. Forschungsgesellschaft für Straßen- und Verkehrswesen e. V. (FGSV), Empfehlungen für das Sicherheitsaudit von Straßen

FGSV-Nr.: 298 / ISBN: -

Ausgabe 2002 / Fassung 2002

Köln 2002

EUSKA. PTV AG, Unfallforschung der Unfallforschung der Versicherer (UDV) Elektronische Unfalltypen-Steckkarte

Version 4.2.0

FIEDLER et al. 1980. Fiedler, J., Hamann, R., Strünkmann, W., Linnenberg E. Innerstädtische Planung als Einflußgröße auf die Verkehrssicherheit, Möglichkeiten zur Verbesserung der Sicherheit an Haltestellen im Straßenraum, Pilotstudie im Auftrag von: Bundesanstalt für Straßenwesen

Bericht zum Forschungsprojekt $7613 / 3$

Bergische Universität Wuppertal

Bergisch Gladbach 1986 
GOOGLE MAPS 2014. Google Inc.

https://maps.google.de/

aufgerufen zwischen 01.06.2014 bis 07.11.2014

GOOGLE EARTH 2014. Google Inc.

https://earth.google.de/

aufgerufen zwischen 01.06.2014 bis 07.11.2014

HAMANN 1985. Hamann R. R.

Fußgängersicherheit an Haltestellen

im Auftrag von: Bundesanstalt für Straßenwesen

Bericht zum Forschungsprojekt 8041

ISSN: 0173-7066

Bergisch Gladbach 1985

HBS 2009. Forschungsgesellschaft für Straßen- und Verkehrswesen e. V. (FGSV), Handbuch für die Bemessung von Straßenverkehrsanlagen

FGSV-Nr.: 299 / ISBN: 3-937356-44-4

Ausgabe 2001 / Fassung 2009

Köln 2009

LERNER 2014. Lerner, M.

Verunglücktenkostensätze, Sachschadenskostensätze, Preisstand 2010

Bundesanstalt für Straßenwesen

unveröffentlicht

LINNENBERG 1988. Linnenberg, E.

Verkehrsgerechte Lage von Haltestellen im Straßenraum unter dem Aspekt der Verkehrssicherheit Dissertation

Fachbereich Bautechnik, Bergische Universität Wuppertal

Wuppertal 1988

M schienengebundener ÖV 2003.

Forschungsgesellschaft für Straßen- und Verkehrswesen e. V. (FGSV),

Merkblatt für die Gestaltung von Anlagen des schienengebundenen öffentlichen Verkehrs

FGSV-Nr.: 249 / ISBN: -

Ausgabe 2003 / Fassung 2003

Köln 2003

PBefG 2013. Bundesrepublik Deutschland, Personenbeförderungsgesetz

Ausfertigungsdatum: 21.03.1961 / zuletzt geändert: 07.08.2013

RAS-Ö, Straßenbahn 1977.

Anlagen des öffentlichen Personennahverkehrs (RAS-Ö), Abschnitt 1: Straßenbahn

Forschungsgesellschaft für Straßen- und Verkehrswesen e. V. (FGSV),

FGSV-Nr.: - / ISBN: 978-3-78120-988-6

Ausgabe 1977 / Fassung 1977

Bonn, Bad Godesberg 1977 
RASt 2006. Forschungsgesellschaft für Straßen- und Verkehrswesen e. V. (FGSV), Richtlinien für die Anlage von Stadtstraßen

FGSV-Nr.: 200 / ISBN: 978-3781216846

Ausgabe 2006 / Fassung 2007

Köln 2007

RiLSA 2010. Forschungsgesellschaft für Straßen- und Verkehrswesen e. V. (FGSV), Richtlinien für Lichtsignalanlagen

FGSV-Nr.: 321 / ISBN: 978-3-939715-91-7

Ausgabe 2010 / Fassung 2010

Köln 2010

\section{StepMap GmbH}

Dienst für Landkartengestaltung StepMap $\mathrm{GmbH}$

Monbijouplatz 5, 10178 Berlin

www.stepmap.de

aufgerufen zwischen 01.06.2014 bis 12.02.2015

StVO 2013. Bundesrepublik Deutschland,

Straßenverkehrs-Ordnung (StVO)

Ausfertigungsdatum: 01.04.2013 / zuletzt geändert: 26.10.2014

TOPP, HAAG et al. 1994.Topp, H. H., Haag, M., Hupfer, C., Ackermann, K.

Haltestellenformen an innerörtlichen Hauptverkehrsstraßen

im Auftrag von: Bundesanstalt für Straßenwesen

Berichte der Bundesanstalt für Straßenwesen, V 12

Bericht zum Forschungsprojekt 4.70318 des Bundesverkehrsministeriums

Verlag für neue Wissenschaft GmbH, Bremerhaven / ISBN: 3-89429-425-6

Bergisch Gladbach, Bremerhaven 1994

TREMOD 2013. ifeu - Institut für Energie- und Umweltforschung Heidelberg GmbH Transport Emission Model (TREMOD)

im Auftrag von: Umweltbundesamt

Version 5.3 (2012)

Datenabruf: 19.06.2013 (Gohlisch, G., Fachgebiet 3.1 Umwelt und Verkehr, Umweltbundesamt) unveröffentlicht

VBG 2011. Verwaltungs-Berufsgenossenschaft (VBG)

Gestaltung von Sicherheitsräumen, Sicherheitsabständen und Verkehrswegen bei Straßenbahnen Version 1.0/2011-04

Hamburg 2011

VDV 2011. Verband Deutscher Verkehrsunternehmen,

VDV-Statistik 2011

Köln

VwV-StVO 2013. Bundesrepublik Deutschland, Allgemeine Verwaltungsvorschrift zur Straßenverkehrs-Ordnung (VwV-StVO)

Ausfertigungsdatum: 22.10.1998 / zuletzt geändert: 11.11.2014 
WEISE, DURTH et al. 1997. Weise, G, Durth, W., Kleinschmidt, P., Lippold, C.

Straßenbau, Planung und Entwurf

3. Auflage, Verlag für Bauwesen

Berlin 1997

WILMS 1998. Wilms, A.

Verkehrssicherheitsbelange bei der Bevorrechtigung von Bussen und Bahnen im Stadtverkehr im Auftrag von: Bundesanstalt für Straßenwesen

Institut für Verkehrswirtschaft, Straßenwesen und Städtebau, Universität Hannover

Hannover 1998 
Anlagen 


\section{Anlagenverzeichnis}

A.1 Zeitreihenvergleich Straßenbahnunfälle und Gesamtverkehrsunfälle innerorts.

A.2 Literaturanalyse obsoleter Regelwerke und Richtlinien

A.3 Tabellarische Zusammenfassung von Regelwerken mit Bezug zur Straßenbahninfrastruktur.

A.4 Tabellarische Übersicht der Städte mit Straßenbahnbetrieb

A.5 Verkehrsunfallanzeige

A.7 Übersicht der Typisierungsmerkmale

A.7.1 allgemeine Typisierungsmerkmale.

A.7.2 zusätzliche Typisierungsmerkmale der Knotenpunkte

A.7.3 zusätzliche Typisierungsmerkmale der Haltstellen

A.7.4 zusätzliche Typisierungsmerkmale der Querungsstellen

A.8 Fotobeispiele der erkannten Defizite der mikroskopischen Analyse . A 35

A.8.1 Knotenpunkte. A35

A.8.1.1 Knotenpunkte mit Lichtsignalanlage ... A 35

A.8.1.2 Knotenpunkte ohne Lichtsignalanlage A 42

A.8.1.3 Kreisverkehrsplätze

A.8.2 Streckenabschnitte zwischen Knotenpunkten nach Lage des Bahnkörpers

A.8.2.1 besonderer Bahnkörper in Mittellage A 50

A.8.2.2 Bahnkörper in Seitenlage A 56

A.8.2.3 Straßenbündige Bahnkörper. A 62

A.8.2.4 Unabhängige Bahnkörper A 66

A.9 ESAS-Defizitcheckliste mit ergänzten Checkpunkten für Straßenbahninfrastrukturen. 
Anlagenband Teil 1,

Datenblätter der Sicherheitsanalyse von Straßenbahnen in ausgewählten Städten und Datenblätter der makroskopischen Analyse

Anlagenband Teil 2,

Dossierbriefe der mikroskopischen Analyse 


\section{A.1 Zeitreihenvergleich Straßenbahnunfälle und Gesamtverkehrsunfälle innerorts}
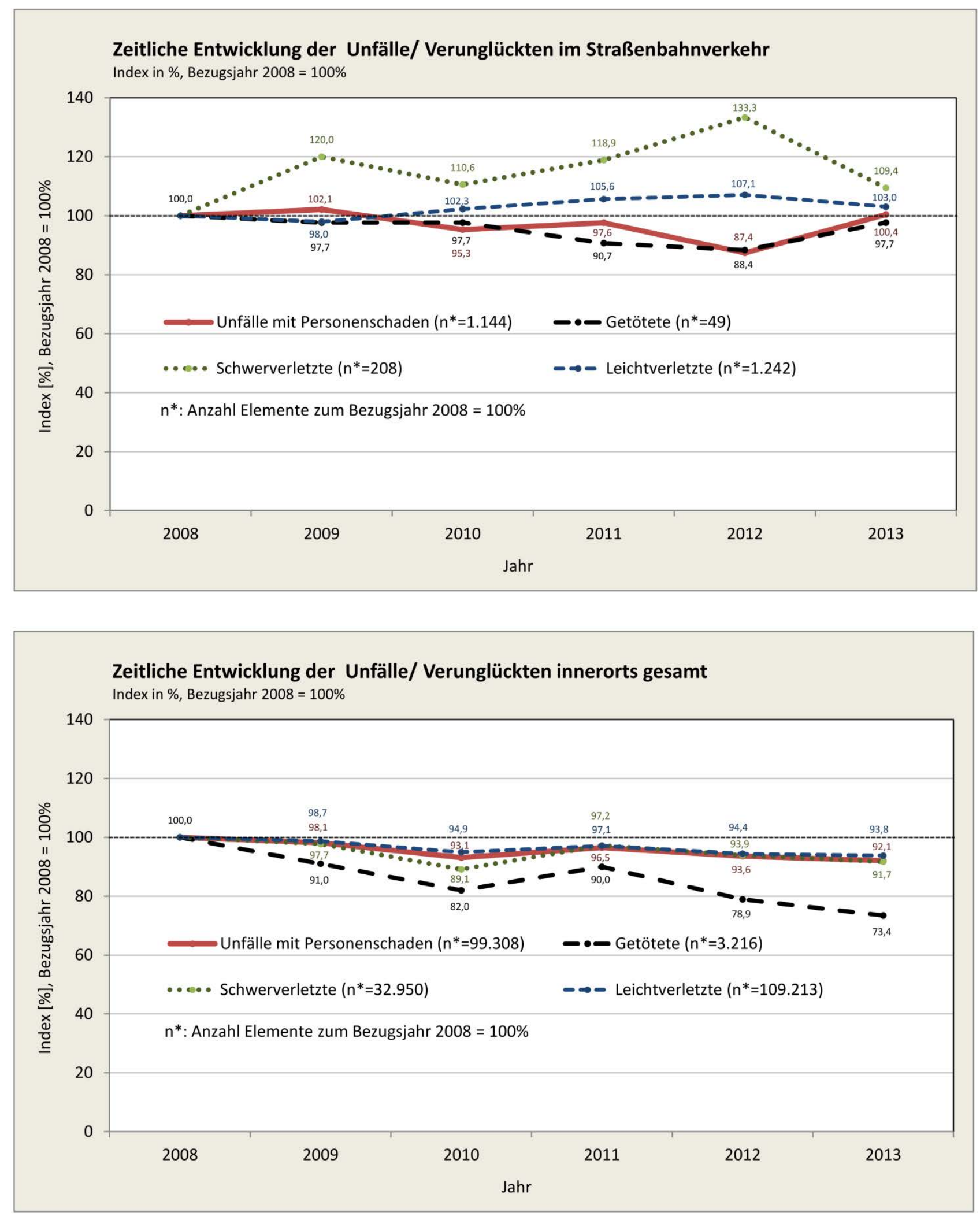

eigene Darstellung,

Datenbasis: Destatis, Straßenverkehrsunfallstatistik, Schienenverkehrsunfallstatistik 


\section{A.2 Literaturanalyse obsoleter Regelwerke und Richtlinien}

\begin{tabular}{|l|l|}
\hline Titel: & Empfehlungen für Anlagen von Hauptverkehrsstraßen \\
\hline Veröffentlicht: & 1993, FGSV \\
\hline Quellenangabe: & [EAHV 1993] \\
\hline
\end{tabular}

Die EAHV behandelte den Entwurf und die Gestaltung angebauter Hauptverkehrsstraßen und anbaufreier Hauptverkehrsstraßen mit plangleichen Knotenpunkten bis zur Einführung der RASt im Jahr 2006.

Im Einführungsschreiben wird darauf hingewiesen, dass die StVO, VwV-StVO sowie die BOStrab im Falle von Unterschieden zur EAHV maßgeblich sind.

Zu den Nutzungsansprüchen an Straßenräume werden in der EAHV folgende Inhalte gegeben ${ }^{111}$ :

- der Bewegungsspielraum für Straßenbahnen ist mit 0,30 m angegeben.

- Der Verkehrsraum in Bögen verbreitert sich ${ }^{112}$.

Die maßgeblichen Richtlinienvorgaben zu den Entwurfselementen der Fahrbahn mit Straßenbahnbetrieb sind folgende ${ }^{113}$ :

- Wendefahrbahnen sollen bei Bahnkörpern der Straßenbahn bei größeren Knotenpunktabständen vorgesehen werden ${ }^{114}$ und sind durch ein gelbes Blinklicht oder besser Hauptsignal (AUS-GELB-ROT-AUS) zu sichern ${ }^{115}$.

Bei Radverkehrsanlagen mit Straßenbahnbetrieb im Straßenraum ist nach EAHV folgendes zu beachten:

- straßenbegleitende Radwege sollen vorgesehen werden, wenn an zweistreifigen Straßen, Straßenbahnen und Kraftfahrzeugen die gleichen Flächen nutzen ${ }^{116}$

- große Fahrradabstellanlagen sind an Endhaltestellen von Straßenbahnen und größeren Einzelhandelsbetrieben anzuordnen ${ }^{117}$

Überquerungsstellen an ÖPNV-Fahrstreifen werden gesondert thematisiert ${ }^{118}$ :

- Überquerungsstellen können als Fahrbahneinengung oder als Zwangsführung mit und ohne LSA (und ggf. gelbes Blinklicht) ausgebildet sein

Wesentliche Richtlinienvorgaben für Straßenbahnen befinden sich im Kapitel zur Führung des ÖPNV ${ }^{119}$ :

- die Führung des ÖPNV ist in Hauptverkehrsstraßen auf ÖPNV-Fahrstreifen und in der Fahrbahn möglich

\footnotetext{
11 [EAHV 1993], S. $19 \mathrm{ff}$

12 [EAHV 1993], Bild 8, S. 22

113 [EAHV 1993], S. 43ff

114 [EAHV 1993], S. 53

115 [EAHV 1993], S. 54

116 [EAHV 1993], S. 85

117 [EAHV 1993], S. 95

118 [EAHV 1993], S. 104f

119 [EAHV 1993], S. 106ff
} 
- Art der Trennung der Verkehrsarten (signaltechnisch, baulich) wird durch unterschiedliche Einflussfaktoren bestimmt (Störungen durch linksabbiegende Kfz, Ein-, Ausparkvorgänge, Halstestellenabstände, Fahrtenfolge, etc.)

- ÖPNV-Fahrstreifen können Mittel- und Seitenlage zugeordnet werden:

- Mittellage für beide Richtungen

- Mittellage für eine Richtung

- Mittellage für beide Richtungen im Wechselbetrieb

- Seitenlage, beidseitig (unbefristet oder zeitlich befristet)

- Seitenlage einseitig (unbefristet, zeitlich befristet, zeitlich wechselnd)

Seitenlage, einseitig für Zweirichtungsbetrieb

- in folgende Bahnkörper wird unterschieden:

- straßenbündiger Bahnkörper ohne räumliche Trennung

- straßenbündiger Bahnkörper mit räumlicher Trennung

- besonderer Bahnkörper mit geschlossenem Oberbau

- besonderer Bahnkörper mit geschottertem Oberbau

- besonderer Bahnkörper mit begrüntem Oberbau

Haltestellen ${ }^{120}$ :

- es wird nach Haltestellentypen unterschieden: Mittellage, Seitenlage, Haltestellekap

- Zuordnung der Wartefläche für Fahrgäste zu den Halteplätzen muss insbesondere unter Sicherheitsaspekten beurteilt werden

Plätze und Knotenpunkte ${ }^{121}$ :

Führung von Straßenbahnen über Kreisverkehrsplätze ohne LSA ist i.d.R. nicht unproblematisch

\begin{tabular}{|l|l|}
\hline Titel: & Empfehlungen für Anlagen von Erschließungsstraßen \\
\hline Veröffentlicht: & 1985, ergänzte Fassung 1995, FGSV \\
\hline Quellenangabe: & [EAE 1995] \\
\hline
\end{tabular}

Behandelte für geplante und bestehende Baugebiete Planung, Entwurf und Gestaltung von Erschließungsstraßen bis zur Einführung der RASt im Jahr 2006.

Erschließungsplan auf Ortsteilebene:

- Netzformen: für versch. Netzformen des Straßennetzes werden Möglichkeiten zur Integration von Straßenbahnlinien aufgezeigt (jedoch ohne Sicherheitsbezug) ${ }^{122}$

- Hinweise zu gebietsspezifischen Netzformen ${ }^{123}$ : bei oberirdischer tangentialer Erschließung von Fußgängerbereichen sollte die Überquerung von stark belasteten Hauptverkehrsstraßen für Fahrgäste vermieden werden

\footnotetext{
${ }^{120}$ [EAHV 1993], S. $117 \mathrm{ff}$

121 [EAHV 1993], S. 140ff

122 [EAE 1995], S. $18 f$

123 [EAE 1995], S. $20 f$
} 


\begin{tabular}{|l|l|}
\hline Titel: & $\begin{array}{l}\text { Richtlinie für die Anlage von Straßen, Anlagen des öffentlichen } \\
\text { Personennahverkehrs, Abschnitt 1: Straßenbahn }\end{array}$ \\
\hline Veröffentlicht: & 1977 \\
\hline Quellenangabe: & [RAS-Ö, Straßenbahn 1977] \\
\hline
\end{tabular}

Behandelte alle Verkehrs- und Betriebsanlagen für Straßenbahnen innerhalb und außerhalb des Verkehrsraumes öffentlicher Straßen für alle verkehrswichtigen Stadtstraßen als auch BundesLand- bzw. Staatsstraßen in bebauten Gebieten.

Grundsätzliches ${ }^{124}$ :

- für die Lage der Gleise gibt es zwei Möglichkeiten: innerhalb (Gleiszone) und außerhalb (besonderer Bahnkörper) der Fahrbahn

- um der Straßenbahn in kritischen Bereichen die ungehinderte Durchfahrt zu gewährleisten, kann es zweckmäßig sein, die Gleiszone durch entsprechende Fahrbahnmarkierungen vom übrigen Verkehr freizuhalten

- enge Straßen mit starkem Verkehr sollten nach Möglichkeit nur mit einer Verkehrsart belegt werden

- eine in beiden Richtungen befahrene Straße mit Gleiszone sollte bei zweigleisigem Betrieb min. zwei Fahrstreifen je Richtung, einschließlich Gleiszone, aufweisen

- zweigleisige Gleiszone ist bei einer vier- und mehrstreifigen Straße mit gerader Fahrspurenzahl in die mittleren Fahrstreifen zu legen

- bei mehr als drei Fahrstreifen sollte die Gleiszone aus Sicherheitsgründen entweder als besonderer Bahnkörper ausgebaut, oder zumindest durch Sperrlinien abgegrenzt sein

- bei Einbahnstraße mit mehr als zwei Fahrstreifen: Gleis im rechten Fahrstreifen oder Haltestelleninsel

- Definition besondere Bahnkörper: außerhalb von Verkehrsraum öffentlicher Straßen oder durch Borde oder bauliche Randeinfassung abgegrenzt

- besondere Bahnkörper fördern Flüssigkeit und Sicherheit des Verkehrsablaufs

- Breite für besonderen Bahnköper sollte sich nach Möglichkeit nach Platzbedarf an Haltestellen und Überquerungsstellen der Fußgänger richten (Vermeidung von Fahrbahneinengungen des KFZ-Verkehrs, Ablagerung von geräumten Schnee, Bepflanzung)

- der Querverkehr besonderer Bahnköper ist auf wenige LSA-Knotenpunkte zu beschränken

- wenn nicht zu vermeiden, dann für Fußgänger ggf. noch zusätzliche Querungen an Haltestellen oder gesonderten LSA Querungen ermöglichen

- Überquerungsstellen mit ausreichend Aufstellfläche auf besonderem Bahnkörper und nach Möglichkeit mit Absperrgitter (Z-Übergang)

- Grundstückserschließungen über besondere Bahnköper in Seitenlage sind nicht zulässig

- Mindestabstände bei Gleisen (Lichtraumprofil) nach BOStrab 1965 werden aufgezeigt ${ }^{125}$

- zur Verhinderung sonstigen Querens werden Absperrungen (Ketten, Geländer) entlang oder innerhalb des besonderen Bahnköpers empfohlen

- bei Kreuzungen und Querungsstellen ist für ausreichend Sicht zu sorgen

- max. Fahrzeugbreite von Straßenbahnen auf öffentlicher Straßen: 2,65 m ${ }^{126}$

124 [RAS-Ö, Straßenbahn 1977], S. 9ff

125 [RAS-Ö, Straßenbahn 1977], Bild4, Bild5, S. $12 f$ 
- Regelbreiten für besondere Bahnkörper werden gegeben ${ }^{127}$; bei Schnellstraßen vergrößern sich die Breiten aufgrund eines größeren Sicherheitsabstands (Bezug zu RAST-Q 1968)

Gleisführung $^{128}$ :

- Linienführung der Gleise möglichst gestreckt

- Kreisbogen-Halbmesser min. 25 m (mit Ausnahme)

- i.d.R. Kreisbögen mit Übergangsbögen und Überhöhung (Bezug zu BOStrab 1965)

- keine Einengung der KFZ-Verkehrsstreifen

- Weichen (insbesondere Zungen) nicht an Fußgängerquerungsstellen

- Verschwenkung von Gleisen aus Mittellage muss übersichtlich und durch Blinklicht oder LSA abgesichert sein

- Wechsel der Gleise von Mittellage auf Seitenlage ist zu vermeiden

Haltestellen ${ }^{129}$ :

- Übersichtliche Stellen sind zu wählen

- Absetzung der Haltestelle von Kreuzung soweit, dass Sichtweiten für Kfz gegeben sind

- paarweise Anordnung von Haltestellen bei starkem Umsteigeverkehr (sonst. bevorzugt vor Knotenpunkt)

- Haltestellen i.d.R. als Haltestelleninsel

- bei starkem Verkehrsaufkommen Querung zur Insel als LSA

- bei Einengung der Fahrbahnbreite an Haltestellen besser Verziehung unter Verringerung der Gehwegbreite

- bei Platzmangel: dynamische Haltestelle (Zeitinsel) möglich

- in schneller befahrenen Bereichen müssen Haltestelleninsel durch Schutzplanken gesichert werden

- Absperrungen zwischen den Gleisen an Haltstellen besser durch Ketten als durch Gitter (Einklemmgefahr durch Fußgänger)

- Schutzdächer dürfen Übersichtlichkeit des Verkehrsraumes nicht beeinträchtigen

- Inselköpfe von Haltestellen sind durch beleuchtete Verkehrszeichen zu kennzeichnen (Bezug zu RAST-K 1973)

\begin{tabular}{|l|l|}
\hline Titel: & Empfehlungen für Anlagen des öffentlichen Personennahverkehrs \\
\hline Veröffentlicht: & 2003, FGSV \\
\hline Quellenangabe: & [EAÖ 2003] \\
\hline
\end{tabular}

Die EAÖ 2003 befassten sich mit dem Entwurf von Fahrweg und Haltestelle und deren Integration in den Straßenraum bis zu deren Fortschreibung (EAÖ 2013).

Rechtsgrundlage für den Betrieb von Straßenbahnen im Sinne des $§ 4$ des PBefG ist die BOStrab vom 11.12.1987.

126 RAS-Ö, Straßenbahn 1977], S. 13

127 RAS-Ö, Straßenbahn 1977], Bild 8, S. 13

128 RAS-Ö, Straßenbahn 1977], S. $16 \mathrm{ff}$

129 RAS-Ö, Straßenbahn 1977], S. $18 \mathrm{ff}$ 
Bei gemeinsamer Führung von Straßenbahn und Kraftfahrzeugverkehr auf einem Fahrstreifen soll nachgewiesen werden, dass der störende Einfluss des Kraftfahrzeugverkehrs durch signaltechnische Maßnahmen weitgehend ausgeschlossen wird ${ }^{130}$. Die Gleise sollen in der Fahrbahnmitte liegen. Bei Straßen mit mehr als zwei Fahrstreifen je Richtung soll der Gleisbereich als besonderer Bahnkörper ausgebaut werden. Bei Einbahnstraßen mit gegenläufigem Straßenbahnverkehr soll das Gleis, das entgegen der Fahrtrichtung in der Straße verläuft, auf einen besonderen Bahnkörper verlegt werden.

Ein eindeutig abgegrenzter ÖPNV-Fahrstreifen muss vorhanden sein, wenn Schnellverkehr der Straßenbahn (> $50 \mathrm{~km} / \mathrm{h}$ ) vorgesehen ist und wenn in Einbahnstraßen ein Zweirichtungsbetrieb der Straßenbahn durchgeführt wird ${ }^{131}$. ÖPNV-Fahrstreifen können zeitlich unbegrenzt oder zeitlich begrenzt für Straßenbahnen oder Linienbusse in Mittel- oder Seitenlage eingerichtet werden. Linienbusse können den vom Kraftfahrzeugverkehr durch Markierung getrennten Gleisbereich oder den besonderen Bahnkörper der Straßenbahn mitbenutzen. Die Breite des Fahrstreifens richtet sich nach dem Fahrzeug mit dem größeren Flächenbedarf. Darüber hinaus ist zu prüfen, ob die Kapazität der Straßenbahnstrecke ausreicht, um den Busverkehr mit aufzunehmen.

Bei der Führung einer Straßenbahn durch den Kreisverkehr ist dem Schienenverkehr Vorrang einzuräumen ${ }^{132}$. Eine Signalisierung der Kreisdurchfahrt wird empfohlen.

Die Sicht der Bus-/Straßenbahnfahrer für gesichertes Anfahren und Verlassen der Haltestellen ist zu gewährleisten und gegebenenfalls durch Hilfsmittel oder durch verkehrsregelnde Maßnahmen zu unterstützen ${ }^{133}$.

An Umsteigehaltestellen zwischen Bussen sowie zwischen Straßenbahnen und Bussen sollen die Haltestellen beider Verkehrsmittel dicht beieinander liegen oder identisch sein. Für die Verbindungswege zwischen beiden Haltestellen sind aus Sicherheitsgründen die Querungen von Fahrbahnen und Gleisen zu minimieren.

Bei Straßenbahnen sind Haltestellenkaps zweckmäßig, wenn die priorisierten Bahnen im Haltestellenbereich mit dem Kraftfahrzeugverkehr auf der gleichen Fläche geführt werden ${ }^{134}$. 


\section{A.3 Tabellarische Zusammenfassung von Regelwerken mit Bezug zur Straßenbahninfrastruktur}

\begin{tabular}{|c|c|c|c|c|}
\hline Kriterium & BOStrab 1987 & StVo 2013 & VwV-StVO 2009 & RASt 2006 \\
\hline \multicolumn{5}{|l|}{ Hierarchie } \\
\hline & $\begin{array}{l}\text { Rechtsgrundlage: } \\
\text { Personenbeförderungs- } \\
\text { gesetz } \\
\text { (§ } 57 \text { PBefG) }\end{array}$ & $\begin{array}{l}\text { Rechtsgrundlage: } \\
\text { Straßenverkehrsgesetz } \\
\text { (§ } 6 \text { Abs. 1 StVG) }\end{array}$ & $\begin{array}{l}\text { Rechtsgrundlage: } \\
\text { Straßenverkehrsgesetz } \\
\text { (§ } 6 \text { Abs. 1 StVG) } \\
\text { vorangestellt: } \\
\text { StVO } 2013\end{array}$ & $\begin{array}{l}\text { FGSV-Regelwerke: R } 1 \\
\text { vorangestellt: } \\
\text { BOStrab } 1987 \\
\text { StVO } 2013\end{array}$ \\
\hline \multicolumn{5}{|l|}{ Strecke } \\
\hline $\begin{array}{l}\text { Differenzier- } \\
\text { ung } \\
\text { Bahnkörper }\end{array}$ & $\begin{array}{l}\text { - straßenbündiger, } \\
\text { - besonderer, } \\
\text { - unabhängiger }\end{array}$ & k.A. & $\begin{array}{l}\text { - ohne eigenen Bahnkörper, } \\
\text { - besonderer, } \\
\text { - unabhängigem } \\
\text { Bahnkörper }\end{array}$ & $\begin{array}{l}\text { - straßenbündiger (mit / } \\
\text { ohne räumliche Trennung) } \\
\text { - besonderer (mit } \\
\text { geschlossenem / } \\
\text { geschottertem / } \\
\text { begrüntem Oberbau) }\end{array}$ \\
\hline \multicolumn{5}{|l|}{ Knotenpunkte } \\
\hline $\begin{array}{l}\text { akzeptierte } \\
\text { Knotenpunkt- } \\
\text { formen }\end{array}$ & $\begin{array}{l}\text { allgem.: durch } \\
\text { Andreaskreuz oder techn. } \\
\text { gesicherte Bahnübergänge }\end{array}$ & k.A. & $\begin{array}{l}\text { - vorfahrtgeregelter } \\
\text { Knotenpunkt } \\
\text { - LSA-Knotenpunkt } \\
\text { - Kreisverkehr mit } \\
\text { vorfahrtsregelnden } \\
\text { Verkehrszeichen für } \\
\text { Straßenbahn }\end{array}$ & k.A. \\
\hline $\begin{array}{l}\text { nicht } \\
\text { akzeptierte } \\
\text { Knotenpunkt- } \\
\text { formen }\end{array}$ & k.A. & k.A. & - Rechts-vor-Links & $\begin{array}{l}\text { - Rechts-vor-Links } \\
\text { - unsignalisierter } \\
\text { Kreisverkehr } \\
\text { - Minikreisverkehr }\end{array}$ \\
\hline \multicolumn{5}{|l|}{ Haltestellen } \\
\hline $\begin{array}{l}\text { Differenzier- } \\
\text { ung } \\
\text { Haltestellen- } \\
\text { formen }\end{array}$ & k.A. & k.A. & k.A. & $\begin{array}{l}\text { - Haltestellenkap } \\
\text { - Mittellage mit } \\
\text { Seitenbahnsteigen } \\
\text { - Mittellage mit } \\
\text { Mittelbahnsteigen } \\
\text { - angehobene Fahrbahn } \\
\text { - Zeitinseln (ggf. mit } \\
\text { angehobener Fahrbahn) }\end{array}$ \\
\hline $\begin{array}{l}\text { Mindestbreite } \\
\text { Bahnsteig }\end{array}$ & $\begin{array}{l}1,50 \text { m (öffentl. } \\
\text { Verkehrsraum, ohne } \\
\text { Sicherheitsraum) }\end{array}$ & k.A. & k.A. & $\begin{array}{l}2,50 \mathrm{~m} \text { (+ Hinweis auf ggf. } \\
\text { Sicherheitsraum zur } \\
\text { Fahrbahn bei Mittellage) }\end{array}$ \\
\hline \multicolumn{5}{|l|}{$\begin{array}{l}\text { Lichtsignal- } \\
\text { steuerung }\end{array}$} \\
\hline Signale & $\begin{array}{l}\text { in Anlage } 4 \text { (Printausgabe) } \\
\text { konkret aufgeführt }\end{array}$ & $\begin{array}{l}\text { für Schienenbahnen können } \\
\text { besondere Zeichen, auch in } \\
\text { abweichenden Phasen } \\
\text { gegeben werden }\end{array}$ & $\begin{array}{l}\text { Verweis: besondere } \\
\text { Zeichen sind in der BOStrab } \\
\text { aufgeführt }\end{array}$ & k.A. \\
\hline
\end{tabular}




\begin{tabular}{|c|c|c|c|c|}
\hline Kriterium & BOStrab 1987 & StVO 2013 & VwV-StVO 2009 & RASt 2006 \\
\hline \multicolumn{5}{|l|}{$\begin{array}{l}\text { Querungs- } \\
\text { stellen }\end{array}$} \\
\hline $\begin{array}{l}\text { Differenzier- } \\
\text { ung } \\
\text { Querungs- } \\
\text { stellen } \\
\text { (mit } \\
\text { konkretem } \\
\text { Bezug zu } \\
\text { Straßenbahn } \\
\text {-infrastruktur) }\end{array}$ & $\begin{array}{l}\text { vgl. § } 20 \text { Bahnübergänge: } \\
\text { - technisch gesichert } \\
\text { - nicht technisch gesichert }\end{array}$ & k.A. & k.A. & $\begin{array}{l}\text { Überquerungsstellen mit } \\
\text { beidseitigen Aufstellflächen } \\
\text { (i.d.R. als Z-Form) mit und } \\
\text { ohne Signalisierung }\end{array}$ \\
\hline \multicolumn{5}{|l|}{ Grundmaße } \\
\hline $\begin{array}{l}\text { Fahrzeug- } \\
\text { maße }\end{array}$ & $\begin{array}{l}\text { - Breite im Höhenbereich } \\
\text { a) bis } 3,4 \text { m über } \\
\text { Schienenoberkante: } 2,65 \\
\text { m, } \\
\text { b) oberhalb von } 3,4 \mathrm{~m} \\
\text { über Schienenoberkante: } \\
2,25 \mathrm{~m} \text {, } \\
\text { - Höhe über } \\
\text { Schienenoberkante bis } \\
\text { Oberkante des } \\
\text { abgezogenen } \\
\text { Stromabnehmers } 4,0 \mathrm{~m} \\
\text { - Länge (bei Zügen im } \\
\text { Straßenverkehr): } 75 \mathrm{~m}\end{array}$ & k.A. & k.A. & $\begin{array}{l}\text { maximaler Fahrzeugbreite } \\
W=2,65 \mathrm{~m}\end{array}$ \\
\hline Lichtraum & $\begin{array}{l}\text { qualitative Definition des } \\
\text { Lichtraums } \\
\text { nähere Angaben/ } \\
\text { Berechnungsvorschrift in } \\
\text { BOStrab-Lichtraum- } \\
\text { Richtlinien (1996) }\end{array}$ & k.A. & k.A. & $\begin{array}{l}\text { Verweis auf BOStrab- } \\
\text { Lichtraum-Richtlinien; } \\
\text { zusätzlich zur } \\
\text { Fahrzeugbreite ist noch ein } \\
\text { Bewegungsspielraum } \\
\text { vorgesehen (der in den } \\
\text { BOStrab-Lichtraum- } \\
\text { Richtlinien jedoch bereits } \\
\text { berücksichtigt ist) }\end{array}$ \\
\hline $\begin{array}{l}\text { Sicherheits- } \\
\text { raum }\end{array}$ & $\begin{array}{l}0,7 \mathrm{~m} \text { breit; } 2,0 \mathrm{~m} \text { hoch; } \\
\text { lotrecht }\end{array}$ & k.A. & k.A. & $\begin{array}{l}\text { Grundmaße für die lichten } \\
\text { Räume ergeben sich, wenn } \\
\text { man zu den } \\
\text { Verkehrsräumen } \\
\text { Sicherheitsräume addiert. }\end{array}$ \\
\hline $\begin{array}{l}\text { Geschwindig } \\
\text {-keit }\end{array}$ & $\begin{array}{l}\text { - straßenbündiger } \\
\text { Bahnkörper: wie übriger } \\
\text { Straßenverkehr } \\
\text { - unabh./ bes. Bahnkörper: } \\
\text { durch technische } \\
\text { Aufsichtsbehörde und } \\
\text { Betriebsleiter } \\
\text { - nähere zur Entwurfs- } \\
\text { geschw. in BOStrab- } \\
\text { Trassierungsrichtlinie }\end{array}$ & $\begin{array}{l}\text { Die zulässige } \\
\text { Höchstgeschwindigkeit } \\
\text { beträgt auch unter } \\
\text { günstigsten Umständen } \\
\text { innerhalb geschlossener } \\
\text { Ortschaften für alle } \\
\text { Kraftfahrzeuge } 50 \mathrm{~km} / \mathrm{h}\end{array}$ & k.A. & $\begin{array}{l}\text { Ein eindeutig abgegrenzter } \\
\text { ÖPNV-Fahrstreifen als } \\
\text { besonderer Bahnkörper } \\
\text { nach } \S 16 \text { Abs. } 6 \text { BOStrab } \\
\text { soll vorhanden sein , wenn } \\
\text { Schnellverkehr der } \\
\text { Straßenbahn }(>50 \mathrm{~km} / \mathrm{h}) \\
\text { vorgesehen ist }\end{array}$ \\
\hline $\begin{array}{l}\text { Brems- } \\
\text { verzögerung }\end{array}$ & $2,73 \mathrm{~m} / \mathrm{s}^{2}(70 \mathrm{~km} / \mathrm{h})$ & k.A. & k.A. & k.A. \\
\hline
\end{tabular}




\begin{tabular}{|c|c|c|c|c|}
\hline Kriterium & RiLSA 2010 & EAÖ 2013 & ERA 2010 & EFA 2002 \\
\hline \multicolumn{5}{|l|}{ Hierarchie } \\
\hline & $\begin{array}{l}\text { FGSV-Regelwerke: R } 1 \\
\text { vorangestellt: } \\
\text { BOStrab } 1987 \\
\text { StVO } 2013\end{array}$ & $\begin{array}{l}\text { FGSV-Regelwerke: R } 2 \\
\text { vorangestellt: } \\
\text { BOStrab } 1987 \\
\text { StVO } 2013 \\
\text { RASt } 2006 \\
\text { RiLSA } 2010\end{array}$ & $\begin{array}{l}\text { FGSV-Regelwerke: R } 2 \\
\text { vorangestellt: } \\
\text { BOStrab } 1987 \\
\text { StVO } 2013 \\
\text { RASt } 2006 \\
\text { RiLSA } 2010\end{array}$ & $\begin{array}{l}\text { A FGSV-Regelwerke: R } 2 \\
\text { vorangestellt: } \\
\text { BOStrab } 1987 \\
\text { StVO } 2013 \\
\text { RASt } 2006 \\
\text { RiLSA } 2010\end{array}$ \\
\hline \multicolumn{5}{|l|}{ Strecke } \\
\hline $\begin{array}{l}\text { Differenzier- } \\
\text { ung } \\
\text { Bahnkörper }\end{array}$ & $\begin{array}{l}\text { - besonderer (S. } 18 \text { mit } \\
\text { Bezug zur BOStrab), } \\
\text { - unabhängiger (S. } 18 \text { mit } \\
\text { Bezug zur BOStrab), } \\
\text { - straßenbündiger }\end{array}$ & $\begin{array}{l}\text { - straßenbündiger, } \\
\text { - besonderer Bahnkörper, } \\
\text { (^ zukünttiger BOStrab } \\
\text { bzw. Entwurf 2013) }\end{array}$ & $\begin{array}{l}\text { • straßenbündiger, } \\
\text { • besonderer, } \\
\text { - unabhängiger }\end{array}$ & $\begin{array}{l}\text { - mit eigenen Bahnkörper / } \\
\text { Gleiskörper, } \\
\text { • ohne eigenen Gleiskörper }\end{array}$ \\
\hline \multicolumn{5}{|l|}{ Knotenpunkte } \\
\hline $\begin{array}{l}\text { akzeptierte } \\
\text { Knotenpunkt- } \\
\text { formen }\end{array}$ & k.A. & k.A. & k.A. & k.A. \\
\hline $\begin{array}{l}\text { nicht } \\
\text { akzeptierte } \\
\text { Knotenpunkt- } \\
\text { formen }\end{array}$ & k.A. & $\begin{array}{l}\text { Verweis auf RASt: } \\
\text { - Rechts-vor-Links } \\
\text { - unsignalisierter } \\
\text { Kreisverkehr } \\
\text { - Minikreisverkehr }\end{array}$ & k.A. & k.A. \\
\hline \multicolumn{5}{|l|}{ Haltestellen } \\
\hline $\begin{array}{l}\text { Differenzier- } \\
\text { ung } \\
\text { Haltestellen- } \\
\text { formen }\end{array}$ & k.A. & $\begin{array}{l}\text { - nach Lage im } \\
\text { Straßenquerschnitt: } \\
\text { Seitenraum oder Insellage } \\
\text { - nach Bauform: } \\
\text { Haltestellenkap, } \\
\text { Seitenbahnsteig } \\
\text { (Haltestelle am } \\
\text { Fahrbahnrand mit oder } \\
\text { ohne Fahrbahnanhebung), } \\
\text { Mittelbahnsteig }\end{array}$ & $\begin{array}{l}\text { - Haltestelle in Mittellage } \\
\text { - Halten auf Fahrbahn } \\
\text { - angehobene Fahrbahn } \\
\text { - Haltestellenkap }\end{array}$ & $\begin{array}{l}\text { - Haltestelle in Mittellage } \\
\text { - mit Mittelinsel } \\
\text { - mit Ausstieg auf die } \\
\text { Fahrbahn } \\
\text { - Haltestelle in } \\
\text { Fahrbahnseitenlage } \\
\text { - am Fahrbahnrand } \\
\text { - Haltestellenkap }\end{array}$ \\
\hline $\begin{array}{l}\text { Mindestbreite } \\
\text { Bahnsteig }\end{array}$ & k.A. & $\begin{array}{l}\text { 2,50 m (+ Sicherheitsraum } \\
\text { zur Fahrbahn bei Insellage); } \\
1,50 \mathrm{~m} \text { Abstand zw. festen } \\
\text { Einbauten und } \\
\text { Bahnsteigkante }\end{array}$ & k.A. & k.A. \\
\hline \multicolumn{5}{|l|}{$\begin{array}{l}\text { Lichtsignal- } \\
\text { steuerung }\end{array}$} \\
\hline Signale & $\begin{array}{l}\text { Verweis auf BOStrab: } \\
\text { waagerechter Balken: Halt; } \\
\text { senkrechter Balken: Frei } \\
\text { ohne bedingt verträgl. } \\
\text { Ströme; Dreieck mit Spitze } \\
\text { nach unten Permissiv- } \\
\text { signal): Frei mit bedingt } \\
\text { verträgl. Strömen; Punkt: } \\
\text { Übergangszeit (Halt zu } \\
\text { erwarten) }\end{array}$ & $\begin{array}{l}\text { - Beschilderung gemäß } \\
\text { StVO und BOStrab } \\
\text { - Signale nach BOStrab }\end{array}$ & $\begin{array}{l}\text { Besondere bzw. } \\
\text { unabhängige Bahnkörper: } \\
\text { ggf. Signalisierung mittels } \\
\text { gelbem Springlicht } \\
\text { gesichert; alternativ: Rot- } \\
\text { Dunkel-Signalisierung }\end{array}$ & k.A. \\
\hline
\end{tabular}




\begin{tabular}{|c|c|c|c|c|}
\hline Kriterium & RiLSA 2010 & EAÖ 2013 & ERA 2010 & EFA 2002 \\
\hline \multicolumn{5}{|l|}{$\begin{array}{l}\text { Querungs- } \\
\text { stellen }\end{array}$} \\
\hline $\begin{array}{l}\text { Differenzier- } \\
\text { ung } \\
\text { Querungs- } \\
\text { stellen } \\
\text { (mit } \\
\text { konkretem } \\
\text { Bezug zu } \\
\text { Straßenbahn } \\
\text {-infrastruktur) }\end{array}$ & k.A. & $\begin{array}{l}\text { - Differenzierung nach } \\
\text { Bauformen: } \\
\text { - Z-Führung/ Führung im } \\
\text { Versatz (mit/ ohne } \\
\text { Signalisierung) } \\
\text { - gradlinige Führung (mit/ } \\
\text { ohne Signalisierung) } \\
\text { - Differenzierung nach Lage } \\
\text { im Netz } \\
\text { - an Knotenpunkten häufig } \\
\text { in Verbindung mit einer } \\
\text { Haltestelle } \\
\text { - an Streckenabschnitten } \\
\text { mit Haltestelle } \\
\text { - an Streckenabschnitten } \\
\text { ohne Haltestelle }\end{array}$ & k.A. & k.A. \\
\hline \multicolumn{5}{|l|}{ Grundmaße } \\
\hline $\begin{array}{l}\text { Fahrzeug- } \\
\text { maße }\end{array}$ & $\begin{array}{l}\text { - pauschale Fahrzeuglänge } \\
\mathrm{I}_{\mathrm{FZ}} \text { Straßenbahn: } 15 \mathrm{~m}\end{array}$ & $\begin{array}{l}\text { Verweis auf BOStrab: } \\
\text { - Breite im Höhenbereich } \\
\text { a) bis } 3,4 \text { m über } \\
\text { Schienenoberkante: } 2,65 \\
\text { m, } \\
\text { b) oberhalb von } 3,4 \mathrm{~m} \\
\text { über Schienenoberkante: } \\
2,25 \mathrm{~m} \text {, } \\
\text { - Höhe über } \\
\text { Schienenoberkante bis } \\
\text { Oberkante des } \\
\text { abgezogenen } \\
\text { Stromabnehmers } 4,0 \mathrm{~m} \text {. } \\
\text { - Länge (bei Zügen im } \\
\text { Straßenverkehr): } 75 \mathrm{~m}\end{array}$ & k.A. & k.A. \\
\hline Lichtraum & k.A. & $\begin{array}{l}\text { Verweis auf BOStrab und } \\
\text { BOStrab-Lichtraum- } \\
\text { Richtlinien; Vorschlag } \\
\text { Lichtraum auf Gerade: Fzg- } \\
\text { breite }+30 \mathrm{~cm})\end{array}$ & k.A. & k.A. \\
\hline $\begin{array}{l}\text { Sicherheitsra } \\
\text { um }\end{array}$ & k.A. & $\begin{array}{l}\text { Verweis auf BOStrab } \S 19 \\
\text { und VBG-Fachinformation } \\
\text { BGI } 5040\end{array}$ & k.A. & k.A. \\
\hline $\begin{array}{l}\text { Geschwindig } \\
\text { keit }\end{array}$ & $\begin{array}{l}\text { Räumzeitberechnung bis } \\
\text { Vmax }=70 \mathrm{~km} / \mathrm{h} \\
\text { angegeben }\end{array}$ & $\begin{array}{l}\text { Verweis auf BOStrab: } \\
\text { - Nach } § 50 \text { setzt } \\
\text { Technische } \\
\text { Aufsichtsbehörde die für } \\
\text { das Streckennetz geltende } \\
\text { Streckenhöchstgeschw. } \\
\text { fest } \\
\text { - straßenbündiger } \\
\text { Bahnkörper: wie übriger } \\
\text { Straßenverkehr } \\
\text { - Entwurfsgeschwindigkeit } \\
\text { für straßenbündige } \\
\text { Bahnkörper i.d.R. } 50 \mathrm{~km} / \mathrm{h} \text {; } \\
\text { für besondere Bahnkörper } \\
\text { i.d.R } 70 \mathrm{~km} / \mathrm{h}\end{array}$ & k.A. & k.A. \\
\hline $\begin{array}{l}\text { Brems- } \\
\text { verzögerung }\end{array}$ & k.A. & k.A. & k.A. & k.A. \\
\hline
\end{tabular}




\section{A.4 Tabellarische Übersicht der Städte mit Straßenbahnbetrieb}

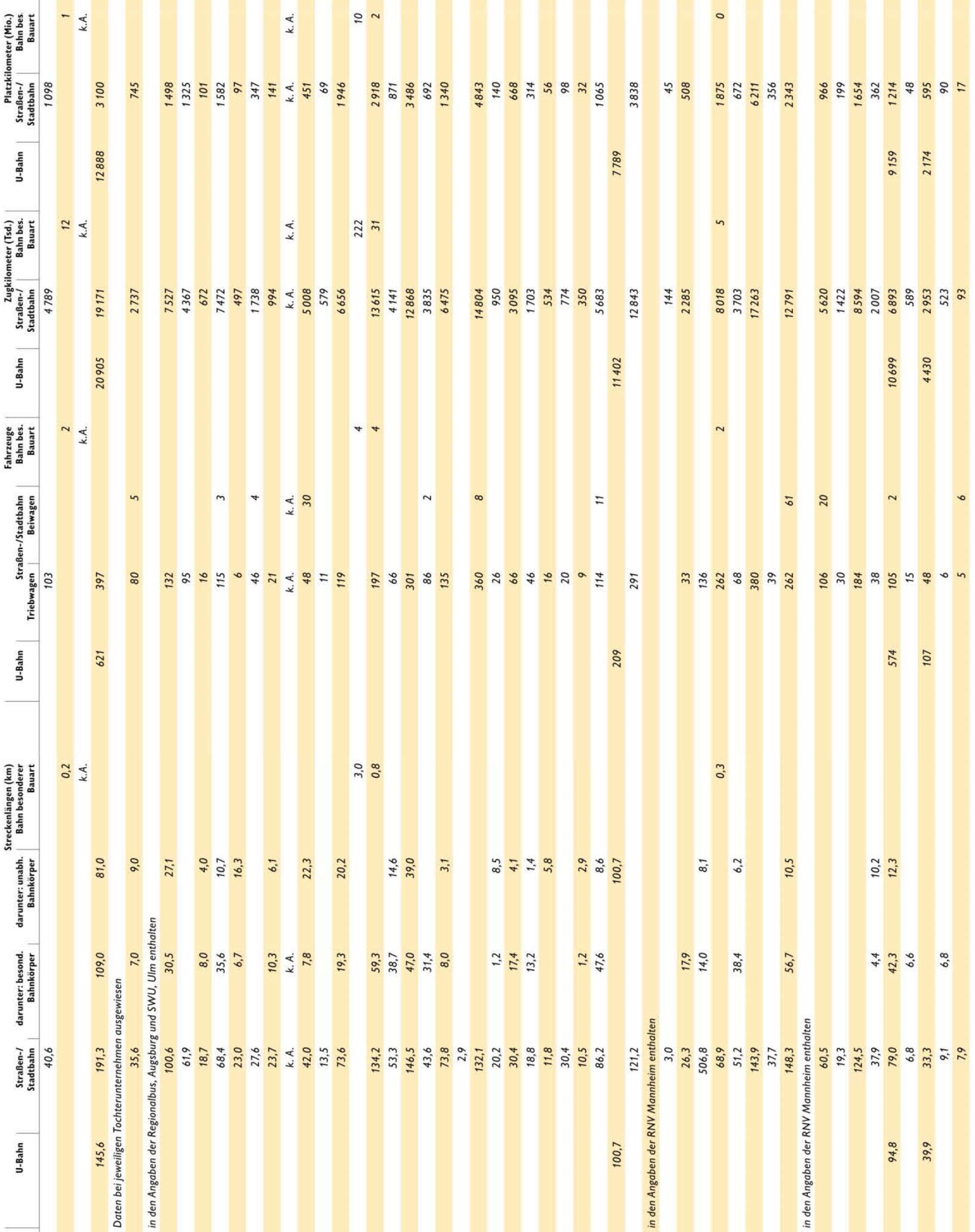

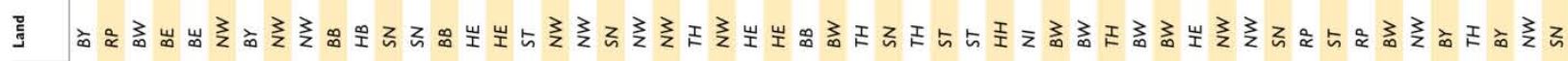

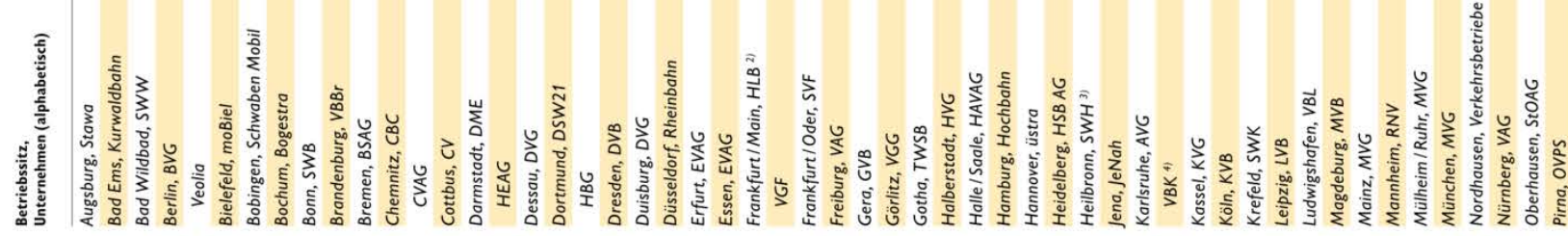




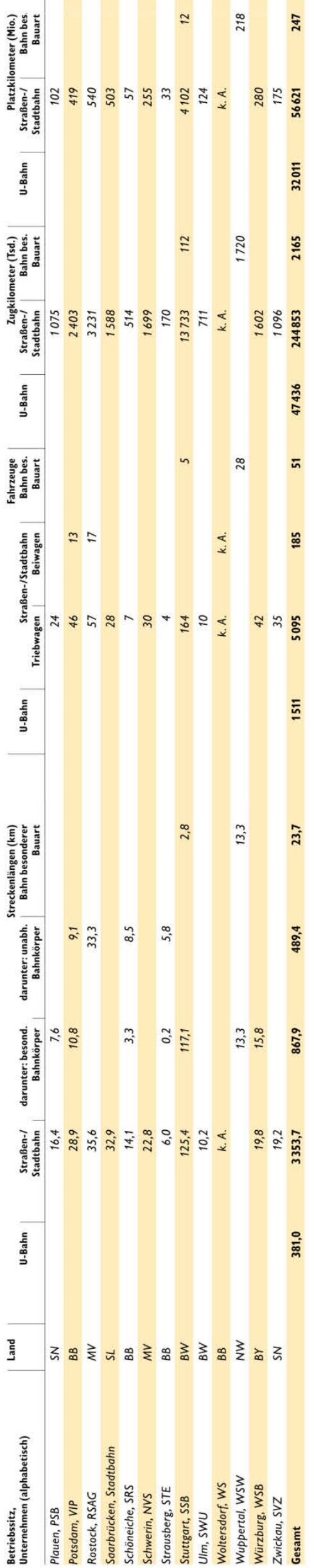




\section{A.5 Verkehrsunfallanzeige}

\section{VERKEHRSUNFALLANZEIGE}

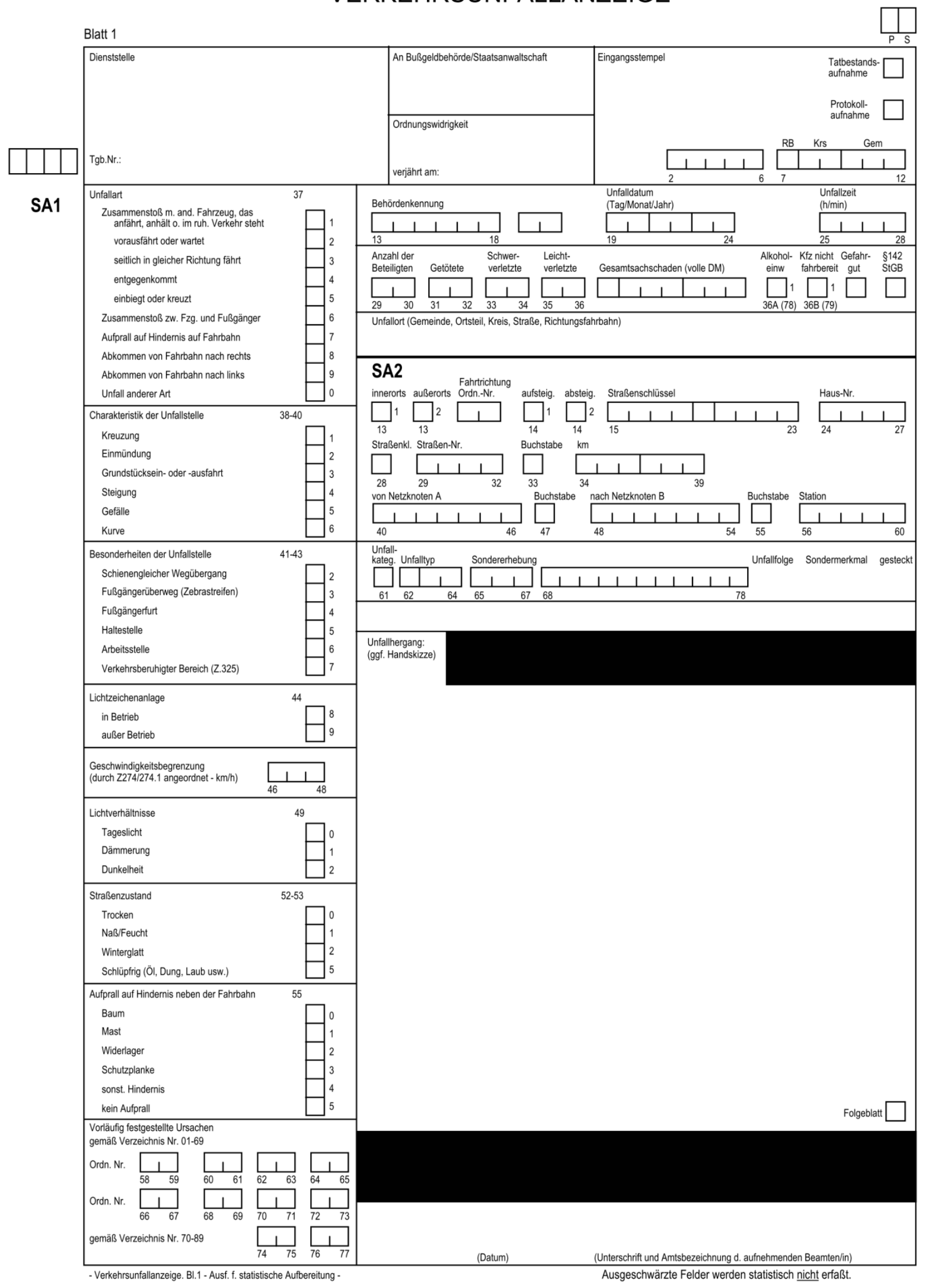




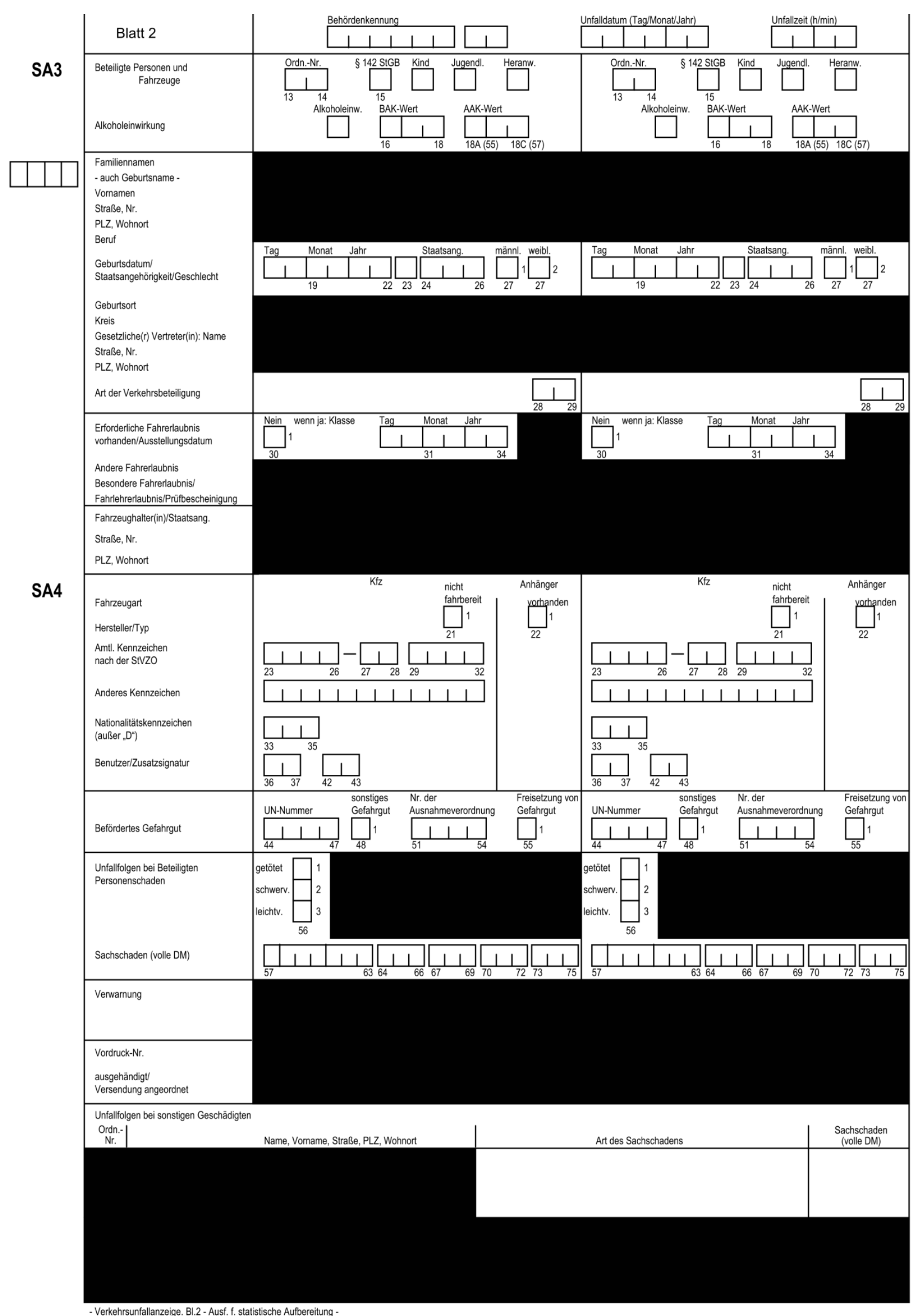




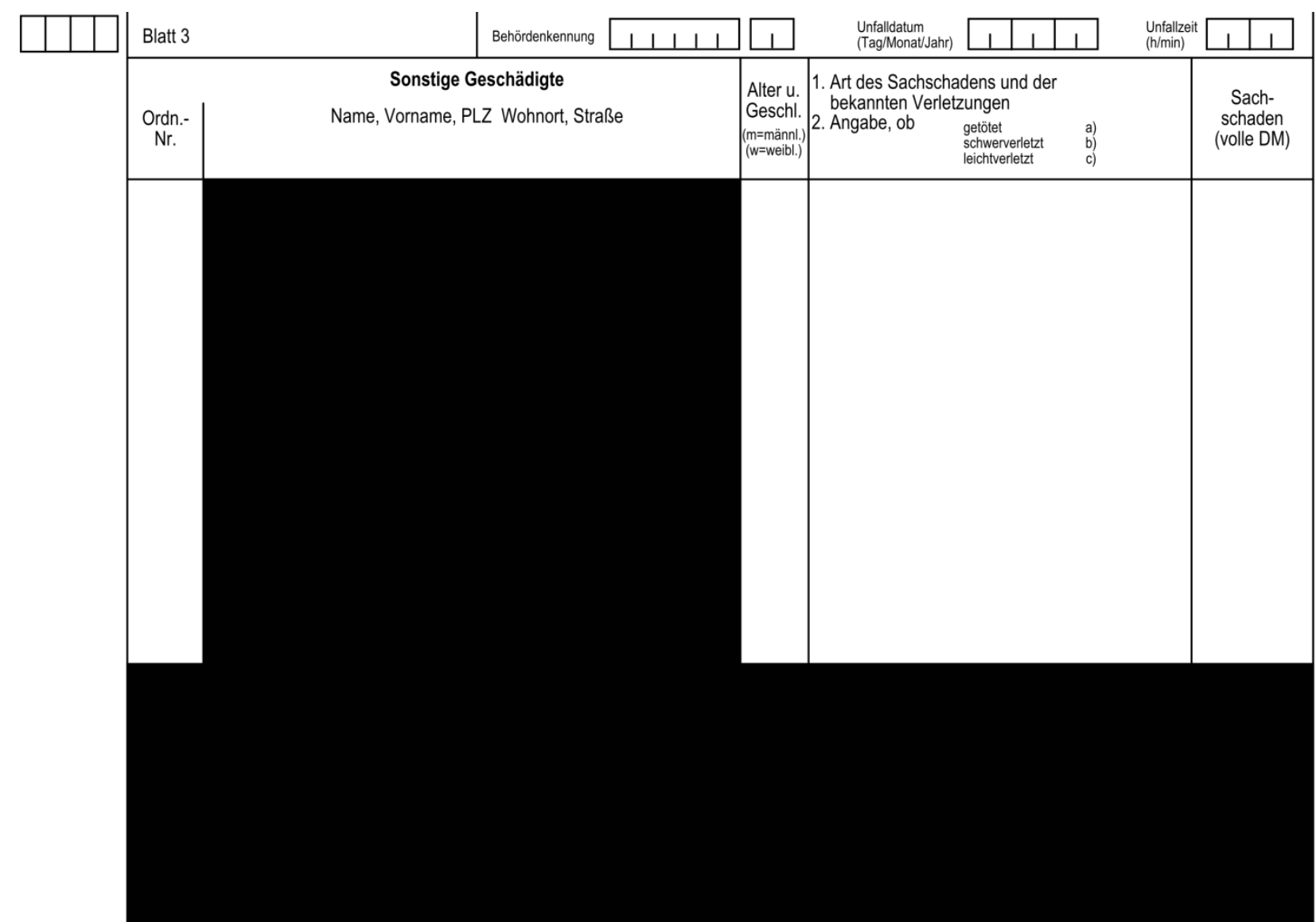

Schäden oder Spuren an Fahrzeugen, die auf den Unfallhergang schließen lassen, techn. Mängel der beteiligten Fahrzeuge unter Angabe der Ordn.-Nr.:

Besonderheiten zur Verkehrslage, zum Unfallort, zur Verkehrsregelung usw., soweit nicht auf Blatt 1 vermerkt:

- Verkehrsunfallanzeige. Bl.3 - Ausf. f. statistische Aufbereitung -

Quelle: [DESTATIS 2013b] 
A.6 Fragebogen Schienenverkehrsunfallstatistik

\section{Statistisches Bundesamt}

\section{Schienenverkehrsunfallstatistik im Jahr 2012}

Statistisches Bundesamt, 65180 Wiesbaden

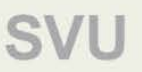

Rücksendung bitte bis 28. März 2013

Ansprechpartnerl-in für Rückfragen
(freiwillige Angabe)

Name:
D!S『A凹S

wissen. nutzen.

Statistisches Bundesam Referat E 306
65180 Wiesbaden
Sie erreichen uns über

Telefon:

Herr Kraski $\quad 061175-2687$ Frau Lerch 0611 75-2535

Telefax: 0611 75-3924

E-Mail: verkehrsunfaelle@destatis.de

Vielen Dank für Ihre Mitarbeit.

Rechtsgrundlagen und weitere rechtliche Hinweise entnehmen Sie der liche Hinweise entnehmen Sie der
beigefügten Unterlage, die Bestandteil beigefügten Unterlage, die Bestandteil
dieses Fragebogens ist. Bitte beachten dieses Fragebogens ist. Bitte beachten die bei der Beantwortung der Fragen

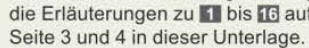

Falls Anschrift oder Firmierung nicht mehr zutreffen, bitte auf Seite 4 korrigieren

Meldung zur Schienenverkehrsunfallstatistik für

- Falls Ihr Unternehmen beispielsweise Eisenbahn- und

I Straßenbahninfrastruktur betreibt, dann sind zwei Frage-

bogen auszufüllen.

Bitte Zutreffendes ankreuzen.

Fahrzeuge nach der Eisenbahn-, Bau- und Betriebsordnung (EBO) oder für Schmalspurbahnen (ESBO)

Fahrzeuge nach der Bau- und Betriebsordnung für Anschlussbahnen (BOA), nur öffentliche Infrastrukturunternehmen gemäß $\S 3$ Abs. 1 Nr. 2 und 3 AEG

Fahrzeuge nach der Straßenbahn-, Bau- und Betriebsordnung (BOStrab)

- Sollten sich im Berichtszeitraum auf Ihrer Infrastruktur keine

I meldepflichtigen Verkehrsunfälle ereignet haben, bitten wir

um einen entsprechenden Hinweis im Feld Bemerkungen auf Seite 4

Entscheidend für die Zuordnung der Fahrzeuge ist, nach welcher Betriebsordnung das Fahrzeug am Unfallort und zum Unfallzeitpunkt betrieben wurde.

A Verunglückte nach Art der Verkehrsbeteiligung (Erläuterungen zu den Verunglückten siehe S. 3)

\begin{tabular}{|c|c|c|c|c|c|c|c|c|c|}
\hline \multirow{3}{*}{$\begin{array}{l}\text { Lfd. } \\
\text { Nr. }\end{array}$} & \multirow{3}{*}{ Art der Verkehrsbeteiligung } & & $\begin{array}{c}\text { Getötete } \\
1\end{array}$ & & $\begin{array}{l}\text { Schwer- } \\
\text { erletzte } 2\end{array}$ & & $\begin{array}{l}\text { Leicht- } \\
\text { erletzte } 3\end{array}$ & & $\begin{array}{l}\text { sammen } 4 \\
\text { alten } 1 \text { bis } 3 \text { ) }\end{array}$ \\
\hline & & \multicolumn{8}{|c|}{ Anzahl } \\
\hline & & & 1 & & 2 & & 3 & & 4 \\
\hline \multicolumn{10}{|c|}{ Fahrer oder Mitfahrer eines/einer } \\
\hline 1 & Personenzuges & 010 & 1,1 & 011 & 1,1 & 012 & 2,1 & 013 & $c_{1}^{1}+1$ \\
\hline 2 & Güterzuges & 014 & 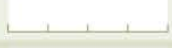 & 015 & 1,1 & 016 & $5,1,1$ & 017 & \\
\hline 3 & sonstigen Eisenbahnfahrzeuges ....... & 018 & $+1, i$ & 019 & $1,1,1$ & 020 & $5+1,1$ & 021 & 1,1 \\
\hline 4 & Straßenbahn & 022 & $1+1$ & 023 & 1,1 & 024 & $\begin{array}{lll}2 & 1 & 1\end{array}$ & 025 & 1 \\
\hline 5 & sonstigen spurgeführten Fahrzeuges & 026 & & 027 & & 028 & & 029 & \\
\hline 6 & Kraftfahrzeuges bzw. Fahrrades ....... & 030 & $2,1,1$ & 031 & $1,1,1$ & 032 & $1,1,1$ & 033 & 1,1 \\
\hline 7 & Fußgänger . & 034 & $5,1,1$, & 035 & $1,1,1$ & 036 & $2,1,1$ & 037 & $1,1,1$ \\
\hline 8 & Andere Personen (z. B. Gleisarbeiter) & 038 & $+, 1,1$, & 039 & $+, 1,1$ & 040 & $5 \quad 1 \quad 1$ & 041 & $1,1,1$ \\
\hline 9 & Insgesamt (Summe 1 bis 8 ) & 042 & $+, 1,1$, & 043 & $+1,1$, & 044 & $+1,1,1$ & 045 & $1,1,1$ \\
\hline svu & & & & & & & & & Seite 1 \\
\hline
\end{tabular}




\section{B Unfälle und Verunglückte nach der Unfallart}

- Als Schienenverkehrsunfälle gelten alle Verkehrsunfälle auf öffentlichen

I Schienenwegen/Strecken, bei denen mindestens ein bewegtes - beim Zusammenprall auch haltendes - schienengebundenes Fahrzeug im Fahrbetrieb (einschließlich Rangierbetrieb) auf den gewidmeten Strecken und Anlagen beteiligt ist. Für die Zuordnung des Unfalls zu einer der nachstehenden Unfallarten ist die erste Unfallart maßgeblich, z. B. bei einem Zusammenstoß zwischen zwei Fahrzeugen mit anschließender Entgleisung ist "Zusammenstoß von Bahnfahrzeugen" anzugeben.

\section{Nicht anzugeben sind}

- Selbstmorde und Selbstmordversuche,

- außergewöhnliche Ereignisse (z.B. Anschläge, Explosionen),

- Unfälle auf dem Betriebsgelände der Bahnen,

- Unfälle auf Strecken vor ihrer Inbetriebnahme (bzw. nach der Stilllegung),

- Unfälle auf Versuchsanlagen, die der Erprobung von Techniken für spurgeführten Verkehr dienen,

- Personenschaden durch „Rempeleien“, Unachtsamkeiten der Mitfahrer, Unfälle beim Ein-/Aussteigen bei stehenden Bahnfahrzeugen etc. oder

- Unfälle, bei denen nur leichter Sachschaden entstand (Sachschaden an Fahrzeugen, Gleisen oder anderen Anlagen unter 10000 Euro) und die keine Störung des Verkehrs (Unterbrechung des Zugverkehrs auf dem Hauptgleis während mehr als sechs Stunden, Umleitung oder Ersatzverkehr von Reisezügen) zur Folge hatten.

\begin{tabular}{|c|c|c|c|c|c|c|c|c|c|c|}
\hline \multirow{3}{*}{$\begin{array}{l}\text { Lfd. } \\
\text { Nr. }\end{array}$} & \multirow{3}{*}{$\begin{array}{l}\text { Unfälle } \\
\text { Verunglückte }\end{array}$} & & \multicolumn{2}{|c|}{ Entgleisungen } & \multicolumn{2}{|c|}{ Zusammenstöße } & \multirow{2}{*}{\multicolumn{2}{|c|}{$\begin{array}{l}\text { Aufpralle auf } \\
\text { Gegenstände }\end{array}$}} & \multirow{2}{*}{\multicolumn{2}{|c|}{$\begin{array}{l}\text { Zusammenpralle } \\
\text { mit Wegebe- } \\
\text { nutzern, z. B. } \\
\text { Fußgängern, Kfz }\end{array}$}} \\
\hline & & & \multicolumn{4}{|c|}{ von Bahnfahrzeugen } & & & & \\
\hline & & & & 1 & & 2 & & 3 & & 4 \\
\hline & Unfälle (Ereignisse) & & & & & & & & & \\
\hline 1 & mit schwerem Sachschaden & [6] & 060 & 1 & 061 & 1 & 062 & $\sqcup$ & 063 & 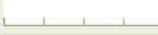 \\
\hline 2 & mit Personenschaden . & 7 & 069 & 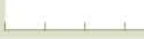 & 070 & & 071 & & 072 & \\
\hline
\end{tabular}

Verunglückte Personen 4

\section{Getötete 1}

3

4

5

7

8

9

10

Leichtverletzte 3

11

13

Fahrgäste

Bahnbedienstete
Fahrgäste

Bahnbedienstete

sonstige Personen

zusammen (Summe 3 bis 5 )
$8 \quad 07$

(9) 087

10

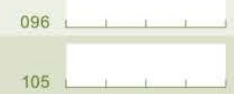

\section{Schwerverletzte [}

sonstige Personen

zusammen (Summe 7 bis 9)

8

114

(9) 123

10

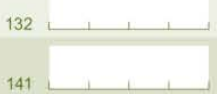

4 zusammen (Summe 11 bis 13)

1 Fahrgäste

2 Bahnbedienstete

13 sonstige Personen
8150

(9) 159

10168

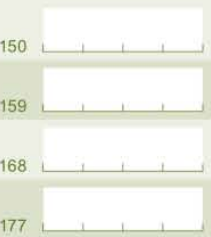

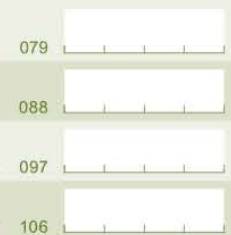

106

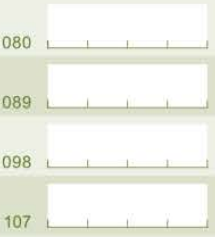

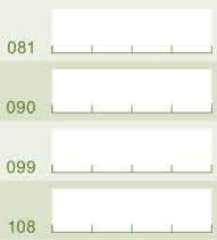

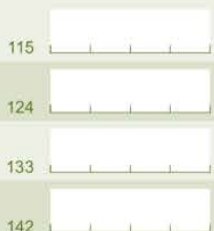

142
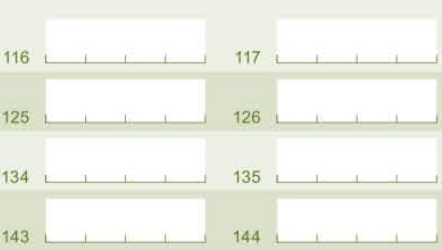
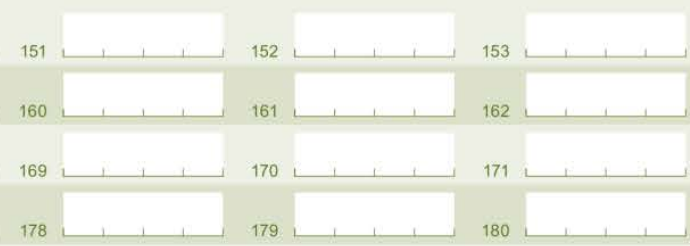


\section{Erläuterungen zu den Abschitten $\mathrm{A}$ und $\mathrm{B}$}

1 Getötete: Personen, die innerhalb von 30 Tagen an den Unfallfolgen starben.

2 Schwerverletzte: Personen, die unmittelbar zur stationären Behandlung (mindestens 24 Stunden) in einem Krankenhaus aufgenommen wurden.

3 Leichtverletzte: Alle übrigen Verletzten

4. Verunglückte: Alle Personen, die beim Unfall verletzt oder getötet wurden.

5 Straßenbahnen: Hierzu zählen auch Stadtbahnen, Hochbahnen, U-Bahnen, Schwebebahnen und ähnliche Nahverkehrsbahnen.

6 Unfälle mit schwerem Sachschaden (keine Personen wurden verletzt oder getötet): Erheblicher Sachschaden an Fahrzeugen, am Gleis oder an anderen Anlagen (i. d. R Schaden in der Höhe von mehr als 10000 Euro) oder Unfälle, die eine beträchtliche Störung des Verkehrs (Unterbrechung des Zugverkehrs auf dem Hauptgleis während mehr als sechs Stunden, Umleitung oder Ersatzverkehr von Reisezügen) zur Folge haben.

7 Unfälle mit Personenschaden: Unfälle bei denen Personen verletzt oder getötet wurden, unabhängig davon ob ein Sachschaden entstanden ist oder nicht.

\begin{tabular}{|c|c|c|}
$\begin{array}{c}\text { Personenunfälle } \\
\mathbf{1 1}\end{array}$ & $\begin{array}{c}\text { Sonstige Unfälle } \\
\mathbf{1 2}\end{array}$ & $\begin{array}{c}\text { Verkehrsunfälle } \\
\text { und Verunglückte } \\
\text { insgesamt } \\
\text { (Spalten 1 bis 6) }\end{array}$ \\
\hline
\end{tabular}

\section{6}

$$
7
$$

8 Fahrgäste: Mit der Bahn fahrende Personen - mit Ausnahme der Bahnbediensteten - einschließlich der Personen, die versuchen auf ein fahrendes Bahnfahrzeug auf- bzw. von einem fahrenden Bahnfahrzeug abzuspringen.

9 Bahnbedienstete sind Arbeitnehmer (auch Rottenarbeiter) von Bahnverkehrs- bzw. Bahninfrastrukturunternehmen, die im Moment des Unfalls im Dienst waren, einschließlich der Beschäftigten von Vertragspartnern.

10 Sonstige Personen: Dies sind alle übrigen Personen, die weder Fahrgäste noch Bahnbedienstete sind, z. B. Wegebenutzer (Fußgänger, Fahrzeugbenutzer)

11 Personenunfälle sind alle Unfälle, an denen Personen beteiligt sind, die nicht als Wegebenutzer gelten, z. B. Unfälle, in die Gleisarbeiter verwickelt sind, oder Personen, die außerhalb eines Bahnübergangs die Gleise überschreiten bzw. auf den Gleisen gehen oder von einem fahrenden Bahnfahrzeug fallen.

12 Sonstige Unfälle: Hierzu zählen alle Verkehrsunfälle, die nicht in den vorhergehenden Spalten genannt worden sind.

13 Bahnübergänge sind durch Andreaskreuze gekennzeichnete höhengleiche Kreuzungen von Eisen-/Straßenbahnen auf unabhängigem Bahnkörper mit Straßen, Wegen und Plätzen (Ausnahmen für Bahnübergänge, die nicht durch Andreaskreuze gekennzeichnet sind, siehe EBO §11, Absatz 3 ).

14 Nicht in den Spalten 1 bis 7 enthalten.

\section{darunter}

Unfälle/Verun-

glückte an Bahnübergängen 13 8

\begin{tabular}{c|c}
$\begin{array}{c}\text { Andere Unfälle } \\
\text { ausgelöst durch } \\
\text { Brand 14 }\end{array}$ & $\begin{array}{c}\text { Lfd. } \\
\text { Nr. }\end{array}$ \\
\hline 9 & \\
\hline
\end{tabular}
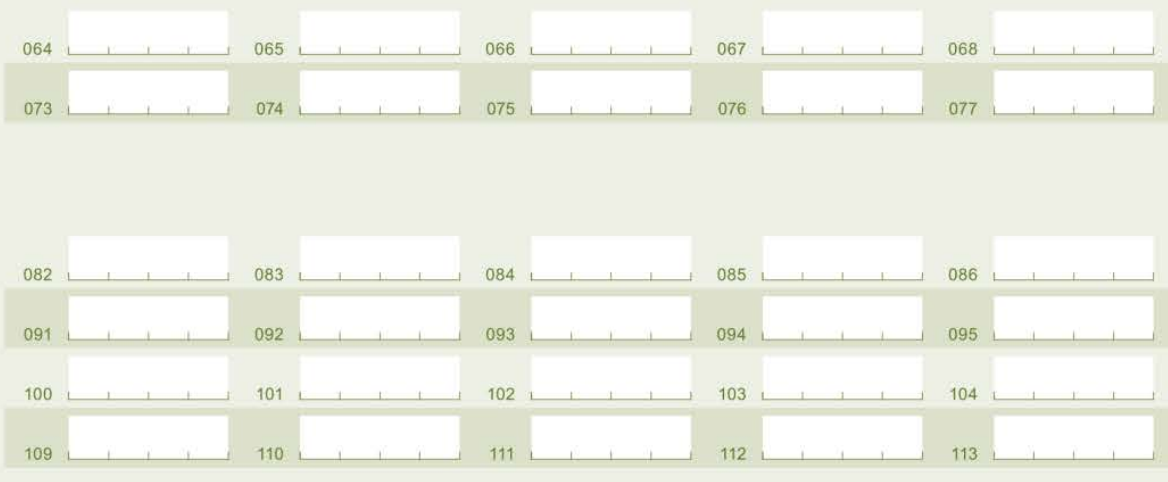

3
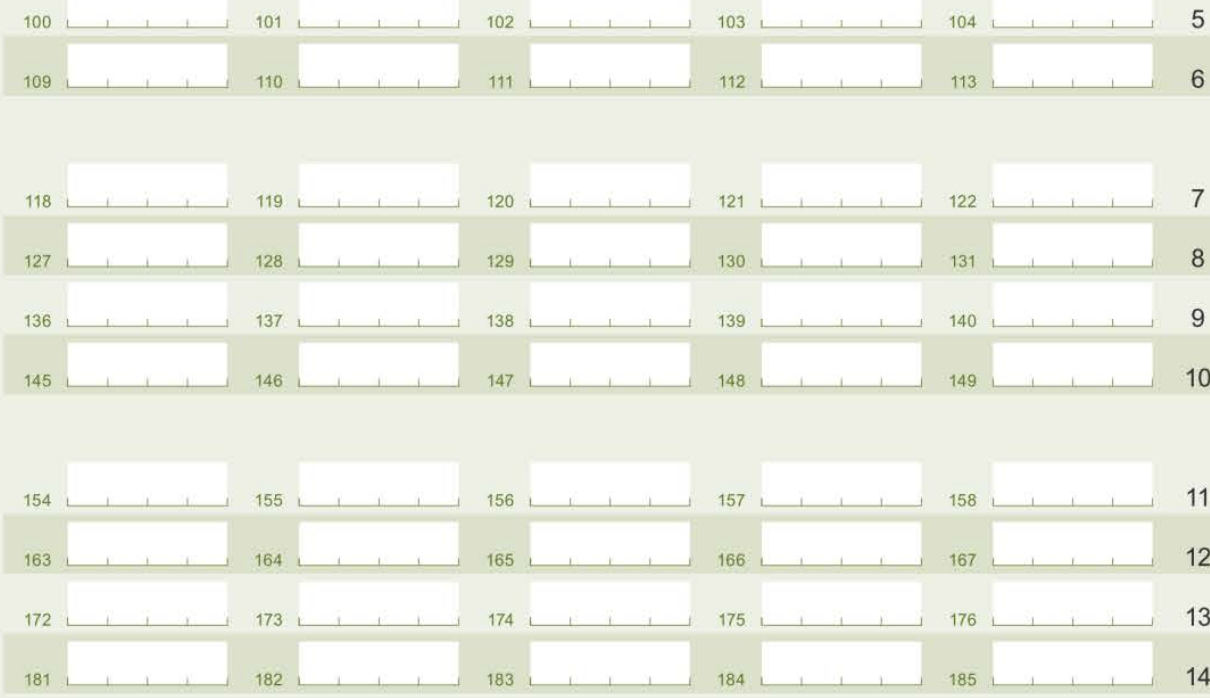
I Für Unfälle beim Transport gefährlicher Güter bitte zusätzlich Teil C ausfüllen.

C Unfälle beim Transport gefährlicher Güter

\begin{tabular}{|c|c|c|c|c|c|c|c|c|c|c|c|c|c|}
\hline \multirow{5}{*}{$\begin{array}{l}\text { Lfd. } \\
\text { Nr. }\end{array}$} & \multirow{5}{*}{$\begin{array}{l}\text { Unfälle beim } \\
\text { Transport } \\
\text { gefährlicher } \\
\text { Güter } 15\end{array}$} & \multirow{4}{*}{\multicolumn{2}{|c|}{ Insgesamt }} & \multicolumn{10}{|c|}{ darunter Unfälle mit Gefahrgutaustritt } \\
\hline & & & & \multirow{3}{*}{\multicolumn{2}{|c|}{ Zusammen }} & \multicolumn{8}{|c|}{ davon } \\
\hline & & & & & & \multicolumn{2}{|c|}{ Entgleisungen } & \multicolumn{2}{|c|}{$\begin{array}{c}\text { Zusammen- } \\
\text { stöße }\end{array}$} & \multirow{2}{*}{\multicolumn{2}{|c|}{$\begin{array}{l}\text { Aufpralle auf } \\
\text { Gegenstände }\end{array}$}} & \multirow{2}{*}{\multicolumn{2}{|c|}{$\begin{array}{c}\text { Übrige } \\
\text { Unfälle } \\
\text { 16 }\end{array}$}} \\
\hline & & & & & & \multicolumn{4}{|c|}{ von Bahnfahrzeugen } & & & & \\
\hline & & & 1 & & 2 & & 3 & & 4 & & 5 & & 6 \\
\hline \multirow[t]{2}{*}{1} & Unfälle mit schwerem & & & \multirow[b]{2}{*}{351} & & \multirow[b]{2}{*}{352} & & \multirow[b]{2}{*}{353} & \multirow[b]{2}{*}{$1 \quad 1$} & \multirow[b]{2}{*}{354} & \multirow[b]{2}{*}{1,1} & \multirow[b]{2}{*}{355} & \\
\hline & Sachschaden ....... & 350 & 2 & & 1,1 & & 1,1 & & & & & & 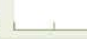 \\
\hline 2 & $\begin{array}{l}\text { Unfälle mit Personen- } \\
\text { schaden }\end{array}$ & 356 & ( & 357 & & 358 & & 359 & & 360 & & 361 & \\
\hline
\end{tabular}

\section{Erläuterungen zum Abschnitt C}

15 Unfälle beim Transport gefährlicher Güter: Unfälle, bei denen ein am Unfall unmittelbar beteiligtes Transportobjekt gefährliche Güter gemäß der „Internationalen Ordnung für die Beförderung gefährlicher Güter mit der Eisenbahn“ (RID) geladen hat.

16 Übrige Unfälle: Dies sind „Zusammenpralle mit Wegebenutzern“, „Personenunfälle“ und „Sonstige Unfälle“.

\section{Bemerkungen}

Zur Vermeidung von Rückfragen unsererseits können Sie hier auf besondere Ereignisse und Umstände hinweisen, die Einfluss auf Ihre Angaben haben.

Bitte zurücksenden an

Statistisches Bundesamt

Referat E 306

65180 Wiesbaden
Bitte aktualisieren Sie Ihre Anschrift, falls erforderlich. Name und Anschrift 


\section{Statistisches Bundesamt}

\section{Schienenverkehrsunfallstatistik im Jahr 2012}

\section{Unterrichtung nach §17 Bundesstatistikgesetz}

\section{Zweck, Art und Umfang der Erhebung}

Die Ergebnisse der vorliegenden Bundesstatistik dienen als Grundlage für verkehrssicherheitspolitische Entscheidungen des Bundes und der Länder. Die Erhebung über Schienenverkehrsunfälle erstreckt sich auf alle Verkehrsunfälle auf öffentlichen Schienenwegen/Strecken, bei denen mindestens ein bewegtes - beim Zusammenprall auch haltendes - schienengebundenes Fahrzeug im Fahrbetrieb auf den gewidmeten Strecken und Anlagen beteiligt ist.

\section{Rechtsgrundlagen}

Verkehrsstatistikgesetz (VerkStatG) in der Fassung der Bekanntmachung vom 20. Februar 2004 (BGBI. I S. 318), zuletzt geändert durch Artikel 2 des Gesetzes vom 6. November 2008 (BGBI. I S. 2162) in Verbindung mit dem Bundesstatistikgesetz (BStatG) vom 22. Januar 1987 (BGBI. I S. 462, 565), zuletzt geändert durch Artikel 3 des Gesetzes vom 7. September 2007 (BGBI. I S. 2246) Erhoben werden die Angaben zu §21 VerkStatG.

\section{Auskunftspflicht}

Die Auskunftsverpflichtung ergibt sich aus $§ 26$ Absatz 1 VerkStatG in Verbindung mit §15 BStatG. Nach §26 Absatz 2 Nummer 8 VerkStatG sind die Inhaber/-innen oder die für die Leitung beziehungsweise Geschäftsführung verantwortlichen Personen der Unternehmen auskunftspflichtig, die Schieneninfrastruktur des öffentlichen Verkehrs im Inland betreiben. Für das Erhebungsmerkmal Zahl der Verunglückten mit Todesfolge (Getötete) sind die Inhaber/-innen oder die für die Leitung beziehungsweise Geschäftsführung verantwortlichen Personen der Unternehmen auskunftspflichtig, die als Betriebsführer oder beauftragte Beförderer öffentliche Personenbeförderung mit Eisenbahnen oder Straßenbahnen beziehungsweise als Frachtführer oder als ausführendes Schienenverkehrsunternehmen Güterverkehr auf dem inländischen Schienennetz des öffentlichen Verkehrs durchführen; die Auskunftspflicht ist erfüllt, wenn sie die Angaben an die Unternehmen weitergeleitet haben, die Schieneninfrastruktur des öffentlichen Verkehrs im Inland betreiben. Gemäß \$15 Absatz 6 BStatG haben Widerspruch und Anfechtungsklage gegen die Aufforderung zur Auskunftserteilung keine aufschiebende Wirkung.

\section{Geheimhaltung}

Die erhobenen Einzelangaben werden nach §16 BStatG grundsätzlich geheim gehalten. Nur in ausdrücklich gesetzlich geregelten Ausnahmefällen dürfen Einzelangaben übermittelt werden.

Eine Übermittlung der erhobenen Angaben ist nach §28 Absatz 1 VerkStatG in Verbindung mit §16 Absatz 4 BStatG an die fachlich zuständigen obersten Bundes- und Landesbehörden in Form von Tabellen mit statistischen Ergebnissen zulässig, auch soweit Tabellenfelder einen einzigen Fall ausweisen.

Nach $\$ 16$ Absatz 6 BStatG ist es auch zulässig, den Hochschulen oder sonstigen Einrichtungen mit der Aufgabe unabhängiger wissenschaftlicher Forschung für die Durchführung wissenschaftlicher Vorhaben Einzelangaben dann zur Verfügung zu stellen, wenn diese so anonymisiert sind, dass sie nu mit einem unverhältnismäßig großen Aufwand an Zeit, Kosten und Arbeitskraft dem Befragten oder Betroffenen zugeordnet werden können. Die Pflicht zur Geheimhaltung besteht auch für Personen, die Empfänger von Einzelangaben sind.

Hilfsmerkmale, Trennen und Löschen, Statistikregister

Der Name und die Anschrift des Eisenbahn- bzw. Straßenbahnunternehmens sowie Name und Telekommunikationsnummern der für Rückfragen zur Verfügung stehenden Person sind Hilfsmerkmale, die lediglich der technischen Durchführung der Erhebung dienen. Sie werden nach Abschluss der Plausibilitätsprüfungen vom Fragebogen abgetrennt, gesondert aufbewahrt und mit Ausnahme des Namens und der Anschrift des Eisenbahn- bzw. Straßenbahnunternehmens spätestens nach Abschluss der nächsten Erhebung vernichtet. Die Angaben zu Namen und Anschrift des Eisenbahn- bzw. Straßenbahnunternehmens können zur Führung des Unternehmensregisters für statistische Verwendungszwecke (Statistikregister) benutzt werden. Rechtsgrundlagen hierfür sind $\S 13$ BStatG und die Verordnung (EG) Nr. 177/2008 des Europäischen Parlaments und des Rates vom 20. Februar 2008 zur Schaffung eines gemeinsamen Rahmens für Unternehmensregister für statistische Zwecke. 


\section{A.7 Übersicht der Typisierungsmerkmale}

\section{A.7.1 allgemeine Typisierungsmerkmale}

\section{Verkehrsstärke der Straßenbahn}

Differenzierungsmerkmal: nach Stundengruppen betreffende Unfälle: Gesamtheit der polizeilichen Unfalldaten mit Personenschaden

\begin{tabular}{|l|c|}
\hline Merkmal (Stundengruppe) & Merkmalsschlüssel/ Merkmalswert \\
\hline $6.00-09.00 \mathrm{Uhr}$ & $\varnothing$ Anzahl der Fzg./ h \\
\hline $9.00-15.00 \mathrm{Uhr}$ & $\varnothing$ Anzahl der Fzg./ h \\
\hline $15.00-19.00 \mathrm{Uhr}$ & $\varnothing$ Anzahl der Fzg./ h \\
\hline $19.00-22.00 \mathrm{Uhr}$ & $\varnothing$ Anzahl der Fzg./ h \\
\hline $22.00-6.00 \mathrm{Uhr}$ & $\varnothing$ Anzahl der Fzg./ h \\
\hline Gesamt: $0.00-24.00 \mathrm{Uhr}$ & $\varnothing$ Anzahl der Fzg./ h \\
\hline
\end{tabular}

\section{Erfassungsmethodik:}

- Auszählen der Verkehrsstärken mittels Fahrpläne der örtlichen Verkehrsbetriebe aus dem Internet

\section{Verkehrsstärke des Kfz-Verkehrs}

Differenzierungsmerkmal: $\varnothing$ Anzahl der Fzg./ h nach Stundengruppen betreffende Unfälle: Unfälle der Detailanalyse

\begin{tabular}{|l|c|}
\hline Merkmal (Stundengruppe) & Merkmalsschlüssel/ Merkmalswert \\
\hline $6.00-09.00 \mathrm{Uhr}$ & $\varnothing$ Anzahl der Fzg./ $\mathrm{h}$ \\
\hline $9.00-15.00 \mathrm{Uhr}$ & $\varnothing$ Anzahl der Fzg./ $\mathrm{h}$ \\
\hline $15.00-19.00 \mathrm{Uhr}$ & $\varnothing$ Anzahl der Fzg./ $\mathrm{h}$ \\
\hline $19.00-22.00 \mathrm{Uhr}$ & $\varnothing$ Anzahl der Fzg./ $\mathrm{h}$ \\
\hline $22.00-6.00 \mathrm{Uhr}$ & $\varnothing$ Anzahl der Fzg./ $\mathrm{h}$ \\
\hline Gesamt: $0.00-24.00 \mathrm{Uhr}$ & $\varnothing$ Anzahl der Fzg./ h \\
\hline
\end{tabular}

\section{Erfassungsmethodik:}

- Anfrage bei den örtlichen Straßenverkehrsbehörden und Straßenbaubehörden mit Bitte um Übersendung der Daten nach Recherche mittels Verkehrsmodellen und vorliegender Zähldaten

- alternativ: Abschätzung der Verkehrsstärke über Kurzzeitzählungen und zugrunde gelegter Tagesganglinien nach HBS 2001, Fassung 2009 


\section{Entwurfssituation}

\section{3a. typische Entwurfssituation nach RASt}

Differenzierungsmerkmal: typische Entwurfssituation in Analogie zu den [RASt 2006] ${ }^{135}$ betreffende Unfälle: Gesamtheit der polizeilichen Unfalldaten mit Personenschaden

\begin{tabular}{|l|c|}
\hline \multicolumn{1}{|c|}{ Merkmal (Entwurfssituation) } & Merkmalsschlüssel/ Merkmalswert \\
\hline Geschäftsstraße & 0 \\
\hline $\begin{array}{l}\text { Verbindungsstraße: gemischte Bebauung } \\
\text { mit mittlerer bis geringer Dichte }\end{array}$ & 1 \\
\hline $\begin{array}{l}\text { Anbaufreie Straße: ohne oder } \\
\text { straßenabgewandte Bebauung }\end{array}$ & 2 \\
\hline Sammelstraße & 3 \\
\hline Quartiersstraße & 4 \\
\hline $\begin{array}{l}\text { Dörfliche Hauptstraße, Örtliche } \\
\text { Einfahrstraße }\end{array}$ & 5 \\
\hline Gewerbestraße, Industriestraße & 6 \\
\hline Sonstige & 7 \\
\hline \multicolumn{2}{|l}{} \\
die grau hinterlegten Felder stellen die Entwurfssituationen dar, in der typischerweise ein \\
Straßenbahnbetrieb zu erwarten ist.
\end{tabular}

\section{Erfassungsmethodik:}

- visuell über Luftbilder und Befahrungsbilder (Google Maps, Bing Maps, Open Street Map)

\section{3b. Geschäftsbesatz von Geschäftsstraßen}

Differenzierungsmerkmal: Anteil des Geschäftsbesatzes in \% betreffende Unfälle: Ausschließlich für Unfälle in Geschäftsstraße für die Gesamtheit der polizeilichen Unfalldaten mit Personenschaden

\begin{tabular}{|l|c|}
\hline \multicolumn{1}{|c|}{ Merkmal (Geschäftsbesatz) } & Merkmalsschlüssel/ Merkmalswert \\
\hline $\begin{array}{l}\text { prozentualer Geschäftsbesatz (Mittel aus } \\
\text { beiden Straßenseiten) bezogen auf } \\
\text { Streckenabschnitt (Knotenpunkt zu } \\
\text { Knotenpunkt) }\end{array}$ & Anteil in \% \\
\hline
\end{tabular}

\section{Erfassungsmethodik:}

- visuell über Luftbilder und Befahrungsbilder (Google Maps, Bing Maps, Open Street Map) 


\section{Querschnittsmerkmale der freien Strecken und Knotenpunktzufahrten}

\section{4a. Querschnittstyp}

Differenzierungsmerkmal: Querschnittstyp in Abhängigkeit der Führung der Straßenbahn und Kfz-Fahrtreifenanzahl

betreffende Unfälle: Gesamtheit der polizeilichen Unfalldaten mit Personenschaden

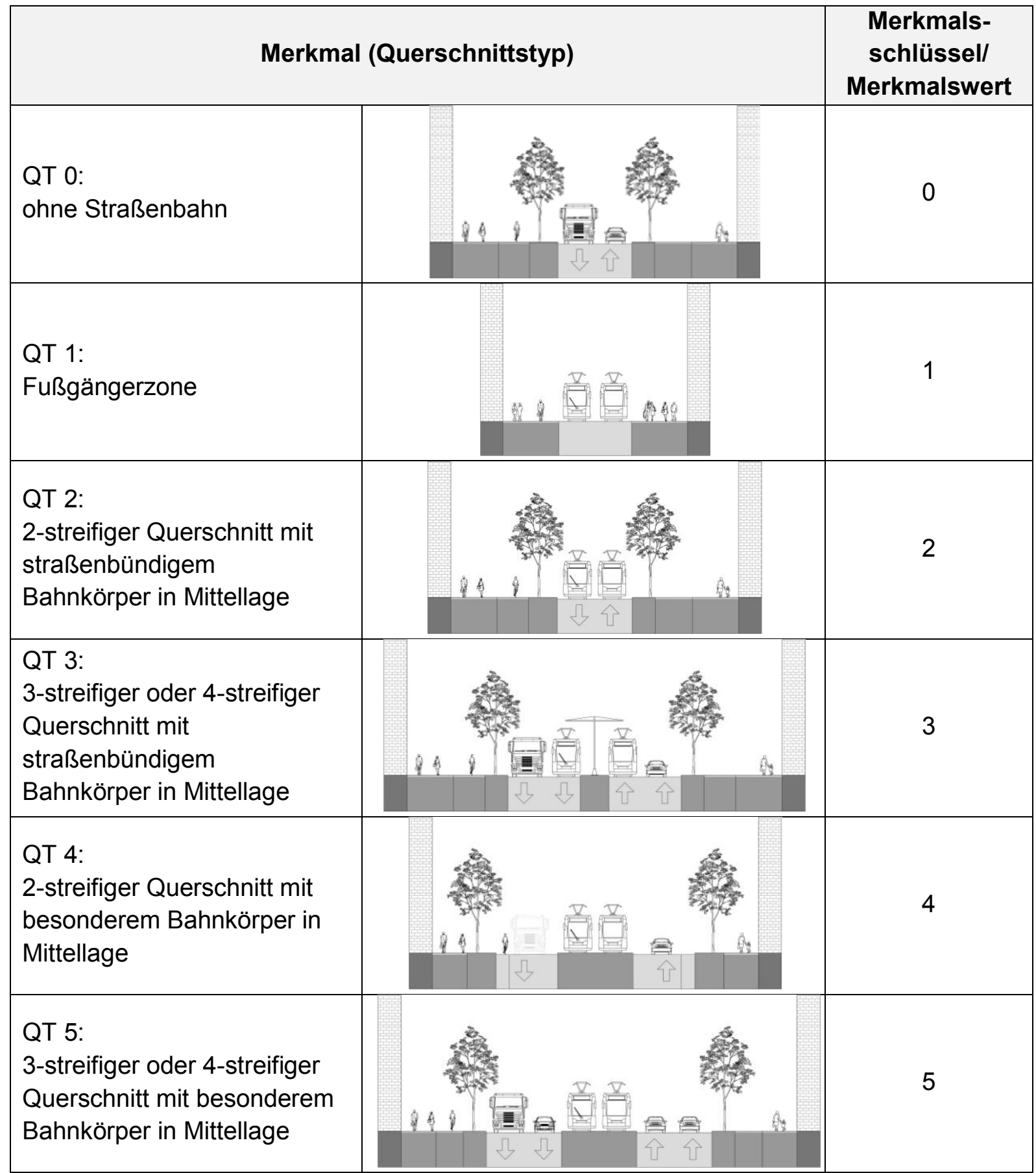




\begin{tabular}{|l|r|c|}
\hline \multicolumn{2}{|c|}{ Merkmal (Querschnittstyp) } & $\begin{array}{c}\text { Merkmals- } \\
\text { schlüssel/ } \\
\text { Merkmalswert }\end{array}$ \\
\hline $\begin{array}{l}\text { QT 6: } \\
\text { besonderer Bahnkörper in } \\
\text { Seitenlage }\end{array}$ & & 6 \\
\hline $\begin{array}{l}\text { QT 7: } \\
\text { unabhängiger Bahnkörper }\end{array}$ & & \\
\end{tabular}

\section{Erfassungsmethodik:}

- visuell über Luftbilder und Befahrungsbilder (Google Maps, Bing Maps, Open Street Map)

\section{Hinweise:}

- an Knotenpunkten ist der Querschnittstyp der betroffenen Knotenpunktzufahrt, in der sich der Unfall ereignet hat, entscheidend.

\section{4b. Führung des Radverkehrs}

Differenzierungsmerkmal: Führung des Radverkehrs im Querschnitt betreffende Unfälle: Gesamtheit der polizeilichen Unfalldaten mit Personenschaden

\begin{tabular}{|l|c|}
\hline \multicolumn{1}{|c|}{ Merkmal (Radverkehrsführung) } & Merkmalsschlüssel/ Merkmalswert \\
\hline $\begin{array}{l}\text { keine Anlagen der Radverkehrs, } \\
\text { Gehweg -Rad frei oder } \\
\text { gemeinsamer Rad-, Gehweg }\end{array}$ & 999 \\
\hline Radverkehr auf der Fahrbahn & 2 \\
\hline Radverkehr im Seitenraum & 1,5 \\
\hline $\begin{array}{l}\text { Unterschiedliche Radverkehrsanlagen auf } \\
\text { beiden Straßenseiten vorhanden und } \\
\text { Fahrtrichtung der Straßenbahn unbekannt }\end{array}$ & 888 \\
\hline $\begin{array}{l}\text { Radverkehrsanlagen auf einer } \\
\text { Straßenseiten vorhanden, auf der anderen } \\
\text { nicht und Fahrtrichtung Straßenbahn } \\
\text { unbekannt }\end{array}$ & \\
\hline
\end{tabular}

\section{Erfassungsmethodik:}

- visuell über Luftbilder und Befahrungsbilder (Google Maps, Bing Maps, Open Street Map) 


\section{Hinweise:}

- an Knotenpunkten ist die Radverkehrsführung der betroffenen Knotenpunktzufahrt, in der sich der Unfall ereignet hat, entscheidend

- ist die Radverkehrsführung der Straßenseiten unterschiedlich, ist (sofern bekannt) die Straßenseite entscheidend, auf der die Straßenbahn gefahren ist

\section{4c. Ruhender Verkehr}

Differenzierungsmerkmal: Form des Parkens im Querschnitt betreffende Unfälle: Gesamtheit der polizeilichen Unfalldaten mit Personenschaden

\begin{tabular}{|l|c|}
\hline \multicolumn{1}{|c|}{ Merkmal (Form des Parkens) } & Merkmalsschlüssel/ Merkmalswert \\
\hline $\begin{array}{l}\text { Keine Anlagen des Ruhenden Verkehrs im } \\
\text { Straßenquerschnitt }\end{array}$ & 999 \\
\hline Längsaufstellung & 2 \\
\hline Schrägaufstellung/ Senkrechtaufstellung & 1,5 \\
\hline $\begin{array}{l}\text { Unterschiedliche Anlagen auf beiden } \\
\text { Straßenseiten vorhanden und Fahrtrichtung } \\
\text { der Straßenbahn unbekannt }\end{array}$ & 888 \\
\hline $\begin{array}{l}\text { Anlage auf einer Straßenseite vorhanden, } \\
\text { auf der anderen nicht und Fahrtrichtung der } \\
\text { Straßenbahn unbekannt }\end{array}$ & 1 \\
\hline
\end{tabular}

\section{Erfassungsmethodik:}

- visuell über Luftbilder und Befahrungsbilder (Google Maps, Bing Maps, Open Street Map)

\section{Hinweise:}

- an Knotenpunkten ist die Form des Parkens der betroffenen Knotenpunktzufahrt, in der sich der Unfall ereignet hat, entscheidend

- ist die Form des Parkens der Straßenseiten unterschiedlich, ist (sofern bekannt) die Straßenseite entscheidend, auf der die Straßenbahn gefahren ist

\section{5. zulässige Höchstgeschwindigkeit des Kfz-Verkehrs}

Differenzierungsmerkmal: zulässige Höchstgeschwindigkeit des Kraftfahrzeugverkehrs in $\mathrm{km} / \mathrm{h}$ betreffende Unfälle: Unfälle der Detailanalyse

\begin{tabular}{|c|c|}
\hline $\begin{array}{c}\text { Merkmal (zulässige } \\
\text { Höchstgeschwindigkeit) }\end{array}$ & Merkmalsschlüssel/ Merkmalswert \\
\hline zulässige Höchstgeschwindigkeit & Geschwindigkeit in km/ h \\
\hline
\end{tabular}

Erfassungsmethodik: - vor Ort Aufnahme des Merkmals 


\section{A.7.2 zusätzliche Typisierungsmerkmale der Knotenpunkte}

\section{Knotenpunktmerkmale}

\section{6a. Knotenpunktabstand im Vorfeld eines Knotenpunktes}

Differenzierungsmerkmal: Knotenpunktabstand vor dem nächsten Knotenpunkt (Abstand zwischen Unfallort und in Fahrtrichtung nachfolgendem Knotenpunkt) betreffende Unfälle: Gesamtheit der polizeilichen Unfalldaten mit Personenschaden

\begin{tabular}{|l|c|}
\hline \multicolumn{1}{|c|}{ Merkmal (Knotenpunktabstand vor KP) } & Merkmalsschlüssel/ Merkmalswert \\
\hline Abstand $\leq 150 \mathrm{~m}$ & Abstand in $\mathrm{m}$ \\
\hline Abstand $>150 \mathrm{~m}$ & 999 \\
\hline
\end{tabular}

\section{Erfassungsmethodik:}

- Messung aus Luftbildern (Google Maps, Bing Maps, Open Street Map)

\section{6b. Knotenpunktabstand nach einem Knotenpunkt}

Differenzierungsmerkmal: Knotenpunktabstand nach einem Knotenpunkt (Abstand zwischen Unfallort und dem in Fahrtrichtung vorgelagertem Knotenpunkt) betreffende Unfälle: Gesamtheit der polizeilichen Unfalldaten mit Personenschaden

\begin{tabular}{|l|c|}
\hline Merkmal (Knotenpunktabstand nach KP) & Merkmalsschlüssel/ Merkmalswert \\
\hline Abstand $\leq 150 \mathrm{~m}$ & Abstand in $\mathrm{m}$ \\
\hline $\begin{array}{l}\text { Abstand }>150 \mathrm{~m} \text {, oder } \\
\text { Unfall unmittelbar auf einem Knotenpunkt } \\
\text { (Knotenpunktabstand vor KP }=0 \text { ) }\end{array}$ & 999 \\
\hline
\end{tabular}

\section{Erfassungsmethodik:}

- Messung aus Luftbildern (Google Maps, Bing Maps, Open Street Map)

\section{Hinweis:}

- befindet sich der Unfall unmittelbar auf einem Knotenpunkt, dann ist der Vorknotenabstand gleich 0 und der Nachknotenabstand gleich 999

\section{6c. Knotenpunkttyp}

Differenzierungsmerkmal: Knotenpunkttyp betreffende Unfälle: Gesamtheit der polizeilichen Unfalldaten mit Personenschaden 


\begin{tabular}{|l|c|}
\hline \multicolumn{1}{|c|}{ Merkmal (Knotenpunkttyp) } & Merkmalsschlüssel/ Merkmalswert \\
\hline $\begin{array}{l}\text { kein Knotenpunkt } \\
\text { (Abstand }>150 \mathrm{~m})\end{array}$ & 999 \\
\hline $\begin{array}{l}\text { Ungesicherter Knotenpunkt } \\
\text { (Rechts-vor-Links) }\end{array}$ & 1 \\
\hline $\begin{array}{l}\text { Knotenpunkt mit Vorfahrt regelnden } \\
\text { Verkehrszeichen }\end{array}$ & 2 \\
\hline Knotenpunkt mit Lichtsignalanlage & 3 \\
\hline Kreisverkehrsplatz & 4 \\
\hline Kreisverkehrsplatz mit Signalisierung & 5 \\
\hline $\begin{array}{l}\text { aufgeweiteter Knotenpunkt mit } \\
\text { Lichtsignalanlage }\end{array}$ & 6 \\
\hline $\begin{array}{l}\text { Grundstückszufahrt } \\
\text { (nur bei: Einbiegen Kreuzen- Abbiege Unfälle) }\end{array}$ & 7 \\
\hline
\end{tabular}

\section{Erfassungsmethodik:}

- visuell über Luftbilder und Befahrungsbilder (Google Maps, Bing Maps, Open Street Map)

\section{Hinweise:}

- ist Abstandsbereich (1m - 50m, 51m - 100m, 101m - 150m) von Vor- und Nachknoten gleich, dann gilt der Vorknotenpunkt, dessen Merkmal aufgenommen wird

\section{6d. Anzahl der Knotenpunktarme}

Differenzierungsmerkmal: Anzahl der Knotenpunktarme betreffende Unfälle: Gesamtheit der polizeilichen Unfalldaten mit Personenschaden

\begin{tabular}{|l|c|}
\hline \multicolumn{1}{|c|}{ Merkmal (Anzahl KP-Arme) } & Merkmalsschlüssel/ Merkmalswert \\
\hline Anzahl der Knotenpunktarme & Anzahl [-] \\
\hline
\end{tabular}

\section{Erfassungsmethodik:}

- visuell über Luftbilder und Befahrungsbilder (Google Maps, Bing Maps, Open Street Map)

\section{Hinweise:}

- ist Abstandsbereich (1m - 50m, 51m - 100m, 101m - 150m) von Vor- und Nachknoten gleich, dann gilt der Vorknotenpunkt, dessen Merkmal aufgenommen wird

- auch Knotenpunktarme mit QT 7 (unabhängiger Bahnkörper) und QT 0 (Querschnitt ohne Straßenbahn) sind zu erfassen 


\section{6e. Anzahl der Fahrstreifen je Knotenpunktarm}

Differenzierungsmerkmal: Anzahl der Fahrstreifen je Knotenpunktarm betreffende Unfälle: Gesamtheit der polizeilichen Unfalldaten mit Personenschaden

\begin{tabular}{|c|c|}
\hline Merkmal (Fahrstreifenanzahl KP-Arme) & Merkmalsschlüssel/ Merkmalswert \\
\hline Anzahl der Fahrstreifen je Knotenpunktarm & Anzahl [-] \\
\hline
\end{tabular}

\section{Erfassungsmethodik:}

- visuell über Luftbilder und Befahrungsbilder (Google Maps, Bing Maps, Open Street Map)

\section{Hinweise:}

- ist Abstandsbereich (1m - 50m, 51m - 100 m, 101 - 150) von Vor- und Nachknoten gleich, dann gilt der Vorknotenpunkt, dessen Merkmal aufgenommen wird

- Merkmalswert = 0 wenn Querschnittstyp 7 (unabhängiger Bahnkörper)

- Merkmalswert $=1$ wenn einstreifige Grundstückszufahrt

\section{6f. Querschnittstyp je Knotenpunktarm}

Differenzierungsmerkmal: Querschnittstyp je Knotenpunktarm in Abhängigkeit der Führung der Straßenbahn und Kfz-Fahrtreifenanzahl betreffende Unfälle: Gesamtheit der polizeilichen Unfalldaten mit Personenschaden

\begin{tabular}{|l|c|c|}
\hline \multicolumn{2}{|c|}{ Merkmal (Querschnittstyp) } & $\begin{array}{c}\text { Merkmals- } \\
\text { schlüssel/ } \\
\text { Merkmalswert }\end{array}$ \\
\hline $\begin{array}{l}\text { QT 0: } \\
\text { ohne Straßenbahn }\end{array}$ & 0 \\
\hline $\begin{array}{l}\text { QT 1: } \\
\text { Fußgängerzone }\end{array}$ & & \\
\hline $\begin{array}{l}\text { QT 2: } \\
\text { 2-streifiger Querschnitt mit } \\
\text { straßenbündigem } \\
\text { Bahnkörper in Mittellage }\end{array}$ & & \\
\hline
\end{tabular}






\section{Erfassungsmethodik:}

- visuell über Luftbilder und Befahrungsbilder (Google Maps, Bing Maps, Open Street Map)

\section{A.7.3 zusätzliche Typisierungsmerkmale der Haltstellen}

\section{Haltestellenmerkmale}

\section{7a. Haltestellenform}

Differenzierungsmerkmal: Haltestellenform betreffende Unfälle: Gesamtheit der polizeilichen Unfalldaten mit Personenschaden

\begin{tabular}{|l|c|}
\hline \multicolumn{1}{|c|}{ Merkmal (Haltestellenform) } & Merkmalsschlüssel/ Merkmalswert \\
\hline $\begin{array}{l}\text { keine Haltestelle } \\
\text { (HS-Anfang/ Ende }>25 \mathrm{~m} \text { ) }\end{array}$ & 999 \\
\hline
\end{tabular}




\begin{tabular}{|l|c|}
\hline \multicolumn{1}{|c|}{ Merkmal (Haltestellenform) } & Merkmalsschlüssel/ Merkmalswert \\
\hline $\begin{array}{l}\text { Seitenlage der Gleise, Wartefläche und } \\
\text { Haltefläche zusammenliegend }\end{array}$ & 2 \\
\hline $\begin{array}{l}\text { Mittellage der Gleise, Wartefläche und } \\
\text { Haltefläche getrennt }\end{array}$ & 3 \\
\hline $\begin{array}{l}\text { Mittellage der Gleise, Wartefläche und } \\
\text { Haltefläche zusammenliegend }\end{array}$ & 4 \\
\hline Komplexhaltestelle & 1,5 \\
\hline $\begin{array}{l}\text { Unterschiedliche Haltestelle auf beiden } \\
\text { Straßenseiten vorhanden und Fahrtrichtung } \\
\text { der Straßenbahn unbekannt }\end{array}$ & 888 \\
\hline $\begin{array}{l}\text { Haltestelle auf einer Straßenseite } \\
\text { vorhanden, auf der anderen nicht und } \\
\text { Fahrtrichtung Straßenbahn unbekannt }\end{array}$ & 2 \\
\hline
\end{tabular}

\section{Erfassungsmethodik:}

- visuell über Luftbilder und Befahrungsbilder (Google Maps, Bing Maps, Open Street Map)

\section{Hinweise:}

- ist die Haltestellenform nach Fahrtrichtung unterschiedlich, ist (sofern bekannt) die Straßenseite entscheidend, auf der die Straßenbahn gefahren ist

\section{7b. Umsteigehaltestelle}

Differenzierungsmerkmal: Umsteigehaltestelle betreffende Unfälle: Gesamtheit der polizeilichen Unfalldaten mit Personenschaden

\begin{tabular}{|l|c|}
\hline \multicolumn{1}{|c|}{ Merkmal (Umsteigehaltestelle) } & Merkmalsschlüssel/ Merkmalswert \\
\hline $\begin{array}{l}\text { Umsteigehaltestellen } \\
\text { (Kreuzung mehrerer Straßenbahnlinien o. Bus-, Zug-, } \\
\text { S-Bahn-Linien) }\end{array}$ & 1 \\
\hline Keine Umsteigehaltestelle & 999 \\
\hline
\end{tabular}

\section{Erfassungsmethodik:}

- visuell über Luftbilder und Befahrungsbilder (Google Maps, Bing Maps, Open Street Map) und Liniennetzpläne

\section{A.7.4 zusätzliche Typisierungsmerkmale der Querungsstellen}

\section{Merkmale von Querungsstellen und Bahnübergängen}

\section{8a. Querungsstellenabstand}

Differenzierungsmerkmal: Querungsstellenabstand nach Entfernungsgruppe 
betreffende Unfälle: Gesamtheit der polizeilichen Unfalldaten mit Personenschaden

\begin{tabular}{|l|c|}
\hline \multicolumn{1}{|c|}{ Merkmal (Entfernungsgruppe) } & Merkmalsschlüssel/ Merkmalswert \\
\hline $\begin{array}{l}\text { kein Bahnübergang/ Querungsanlage } \\
\text { (Querungsstellenabstand >100m) }\end{array}$ & 999 \\
\hline $\begin{array}{l}\text { Entfernungsgruppe 0 } \\
\text { Unfall unmittelbar an Querungsanlage }(\leq 5 \mathrm{~m})\end{array}$ & 0 \\
\hline $\begin{array}{l}\text { Entfernungsgruppe 1 } \\
\text { ( } 25 \mathrm{~m} \text { entfernt vom Unfallort) }\end{array}$ & 1 \\
\hline $\begin{array}{l}\text { Entfernungsgruppe 2 } \\
(\geq 25 \mathrm{~m} \text { - 50m entfernt vom Unfallort) }\end{array}$ & 2 \\
\hline $\begin{array}{l}\text { Entfernungsgruppe 3 } \\
(\geq 50 \mathrm{~m} \text {-100m entfernt vom Unfallort) }\end{array}$ & 3 \\
\hline
\end{tabular}

\section{Erfassungsmethodik:}

- Messung aus Luftbildern (Google Maps, Bing Maps, Open Street Map)

\section{8b. Form der Querungsanlage}

Differenzierungsmerkmal: Form der Querungsanlage betreffende Unfälle: Gesamtheit der polizeilichen Unfalldaten mit Personenschaden

\begin{tabular}{|c|c|}
\hline Merkmal (Form der Querungsanlage) & Merkmalsschlüssel/ Merkmalswert \\
\hline Lichtsignalanlage & $\begin{array}{ll}999 & =\text { nicht vorhanden } \\
1 & =\text { vorhanden } \\
2 & =\text { LSA außer Betrieb }\end{array}$ \\
\hline Schranke & $\begin{array}{l}999=\text { nicht vorhanden } \\
1=\text { vorhanden }\end{array}$ \\
\hline Z-Übergang & $\begin{array}{l}999=\text { nicht vorhanden } \\
1=\text { vorhanden }\end{array}$ \\
\hline $\begin{array}{l}\text { Gesicherte Querungsanlage am } \\
\text { Knotenpunkt }\end{array}$ & $\begin{array}{l}999=\text { nicht vorhanden } \\
1=\text { vorhanden } \\
2 \quad=\text { LSA außer Betrieb }\end{array}$ \\
\hline Sonstige (ungesichert o. nicht zuordenbar) & $\begin{array}{l}999=\text { nicht vorhanden } \\
1 \quad=\text { vorhanden }\end{array}$ \\
\hline
\end{tabular}

\section{Erfassungsmethodik:}

- visuell über Luftbilder und Befahrungsbilder (Google Maps, Bing Maps, Open Street Map) 


\section{A.8 Fotobeispiele der erkannten Defizite der mikroskopischen Analyse}

\section{A.8.1 Knotenpunkte}

\section{A.8.1.1 Knotenpunkte mit Lichtsignalanlage}

\section{Fußgänger}

(Knotenpunkte mit Lichtsignalanlage)

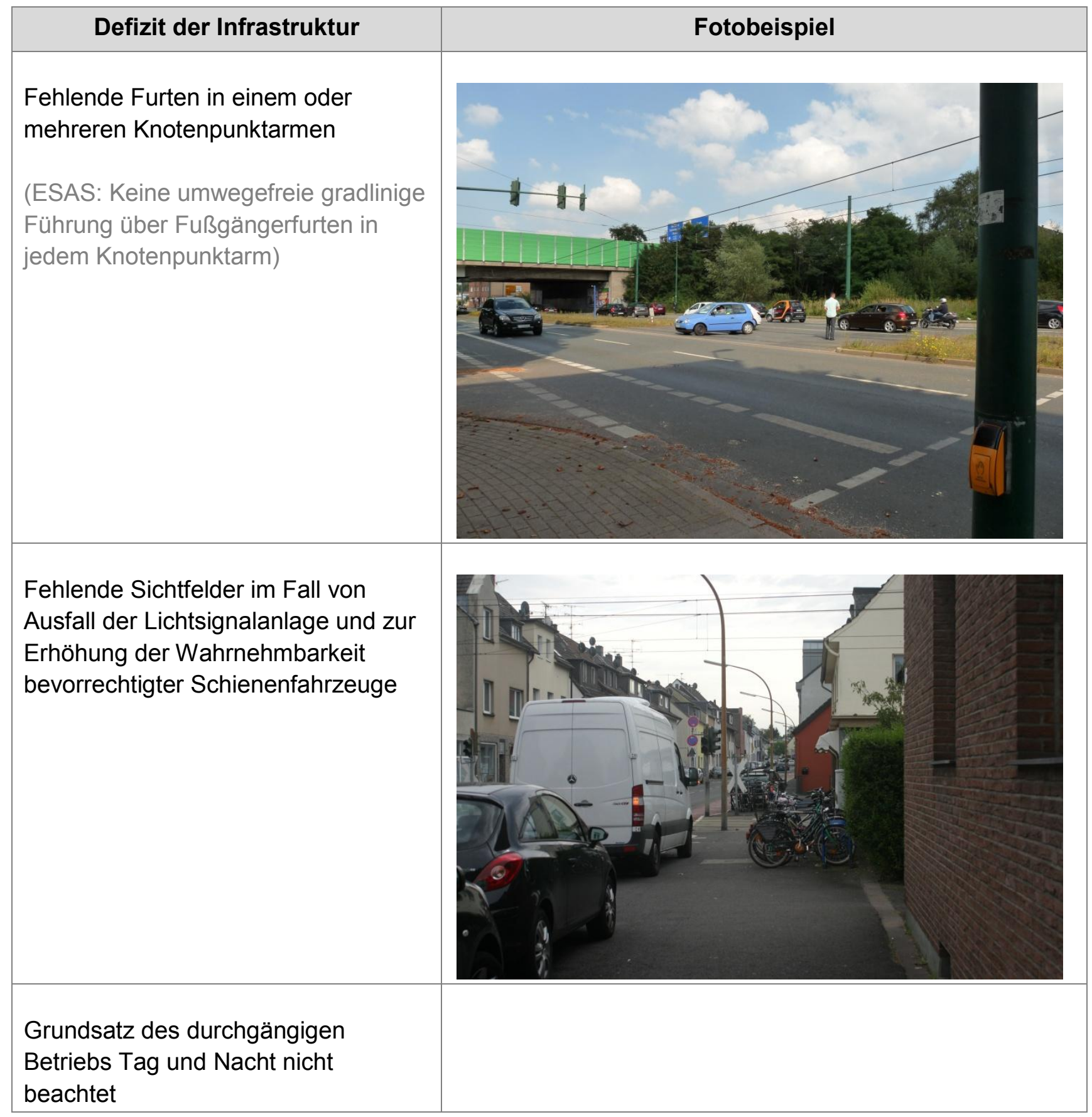




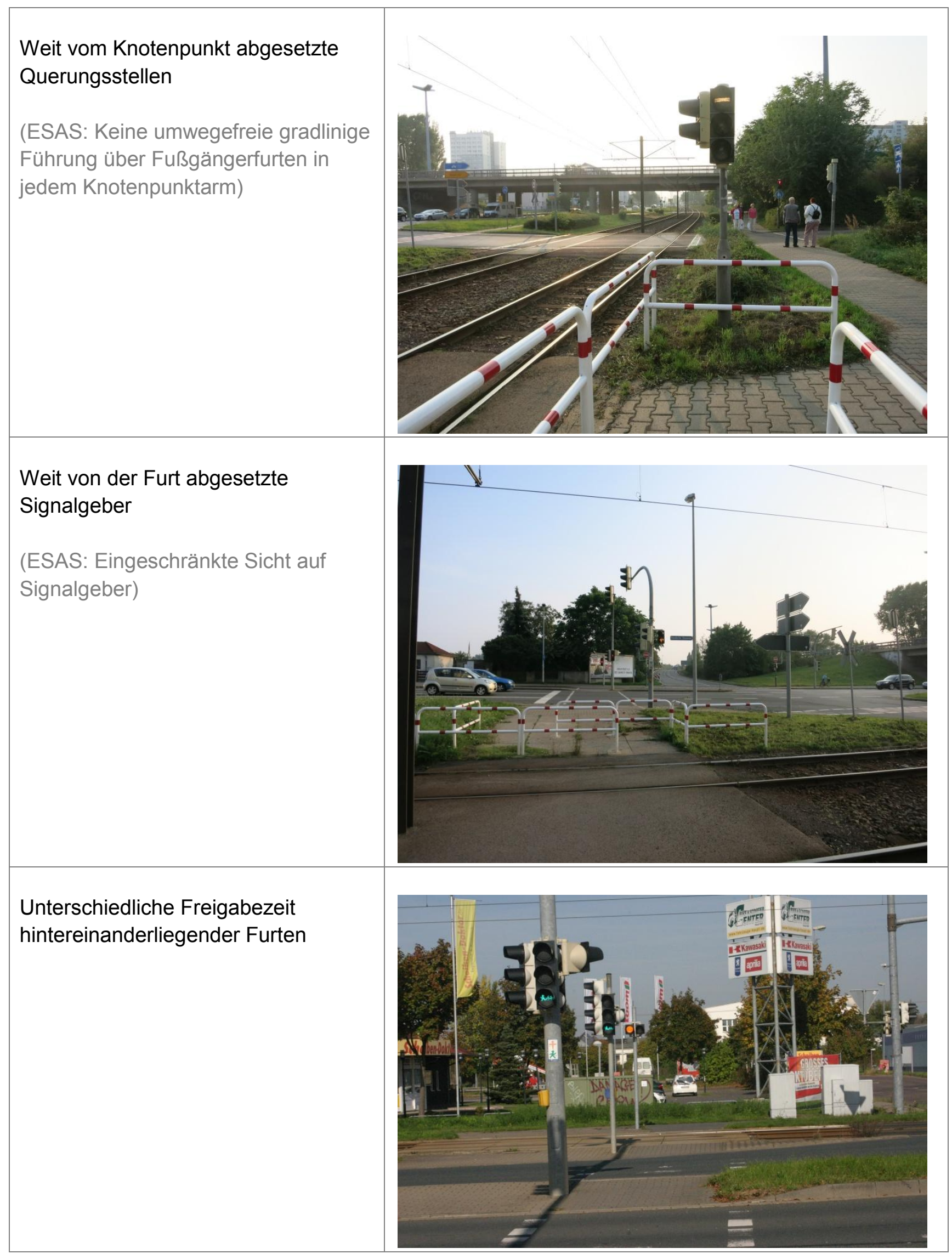




\section{Radfahrer}

(Knotenpunkte mit Lichtsignalanlage)

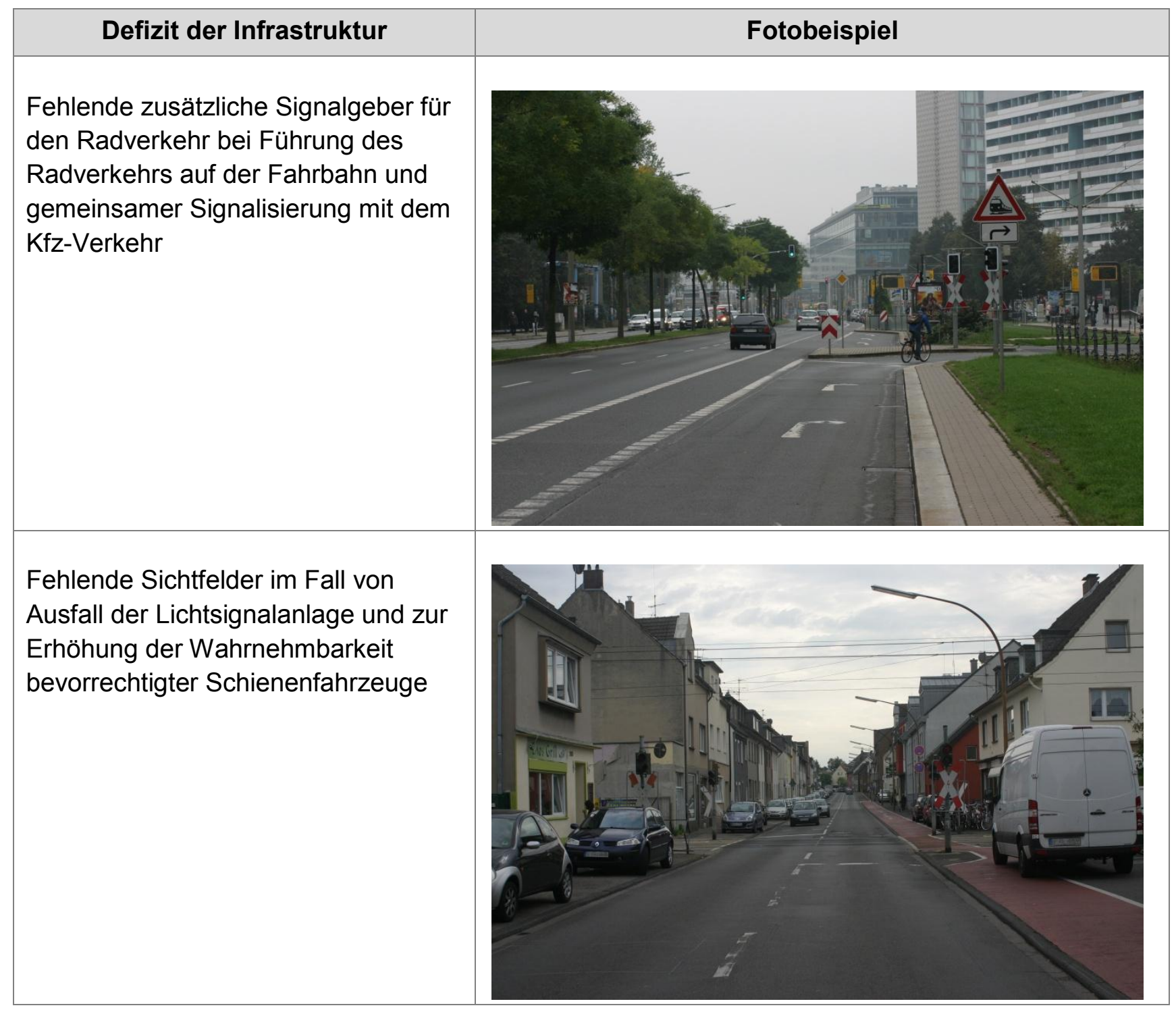




\section{Kraftfahrzeuge}

(Knotenpunkte mit Lichtsignalanlage)

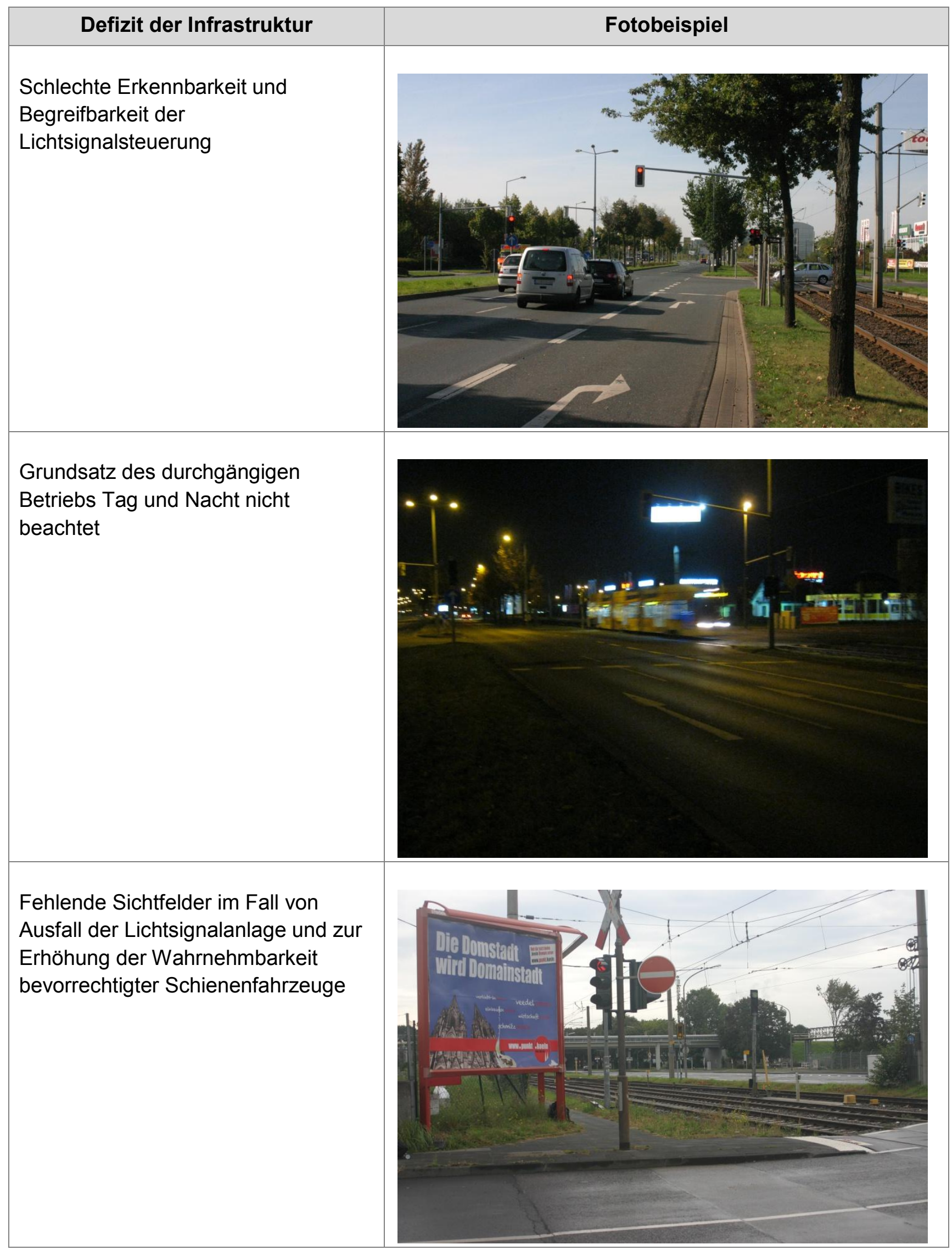




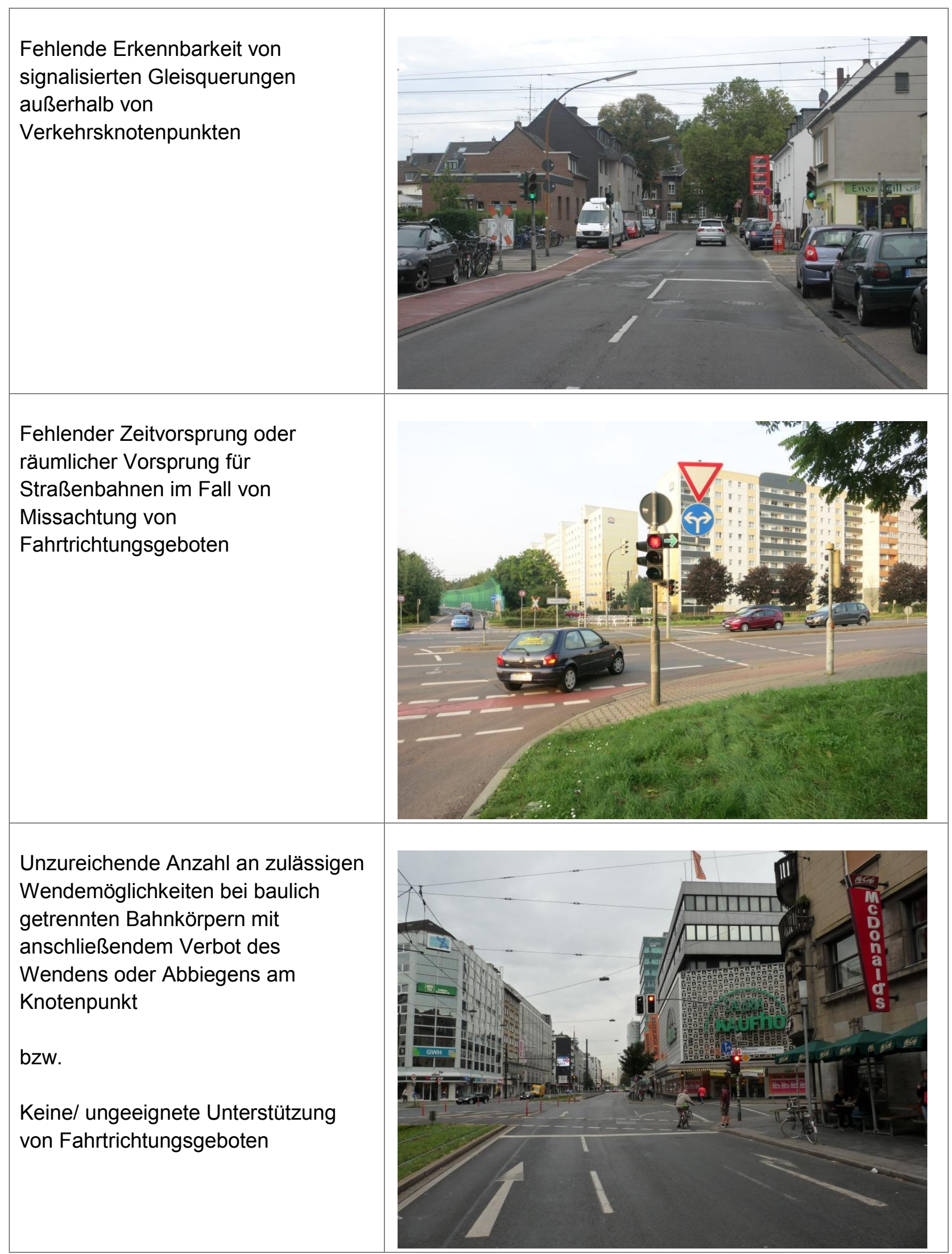




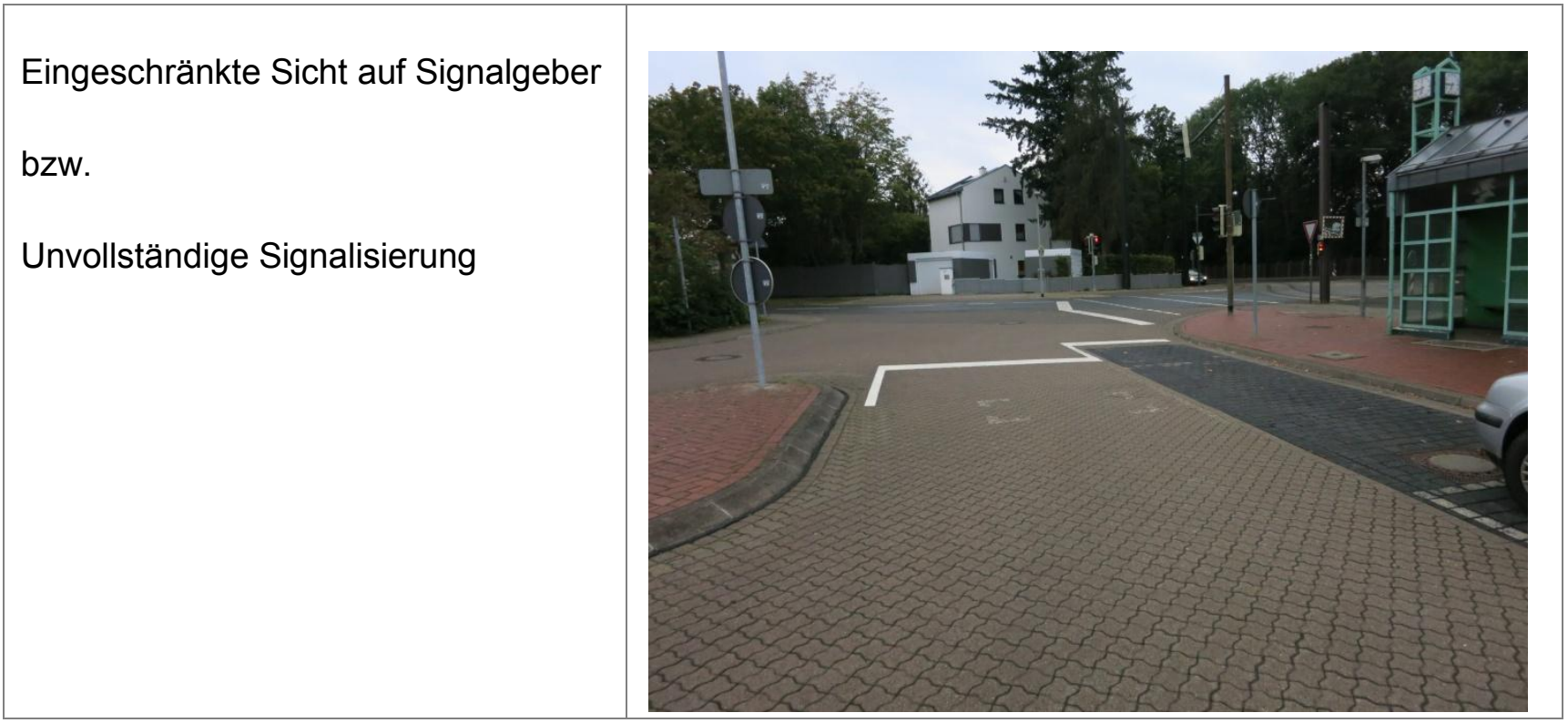




\section{Besonderheiten an Haltestellen}

(Knotenpunkte mit Lichtsignalanlage)

\begin{tabular}{|l|c|c|}
\hline \multicolumn{1}{|c|}{ Defizit der Infrastruktur } & \multicolumn{2}{|c|}{ Fotobeispiel } \\
\hline $\begin{array}{l}\text { Fehlende } \\
\text { geschwindigkeitsdämpfende } \\
\text { Elemente für Radfahrer an Rampen } \\
\text { von Hochbahnsteigen }\end{array}$ & (n) &
\end{tabular}


A.8.1.2 Knotenpunkte ohne Lichtsignalanlage

\section{Fußgänger}

(Knotenpunkte ohne Lichtsignalanlage)

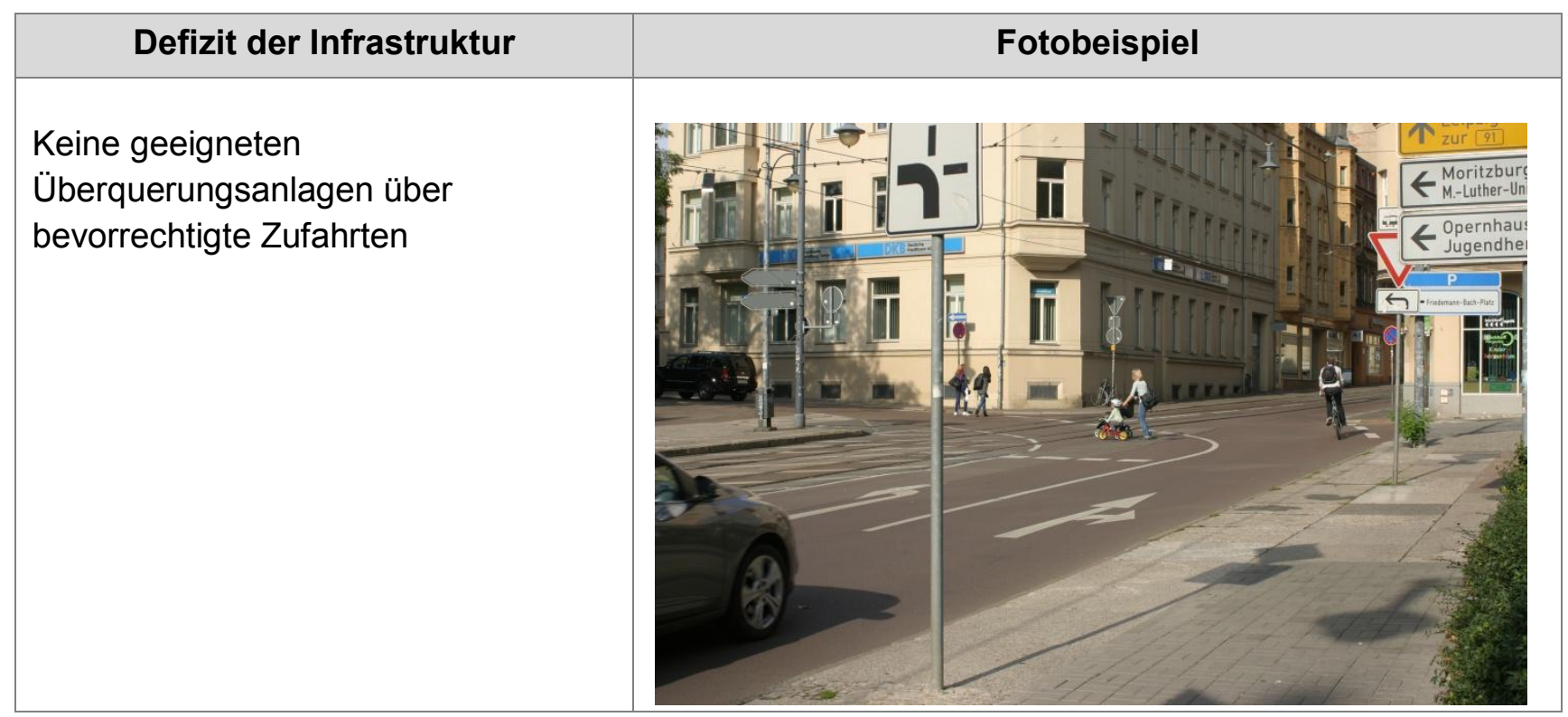




\section{Radfahrer}

(Knotenpunkte ohne Lichtsignalanlage)

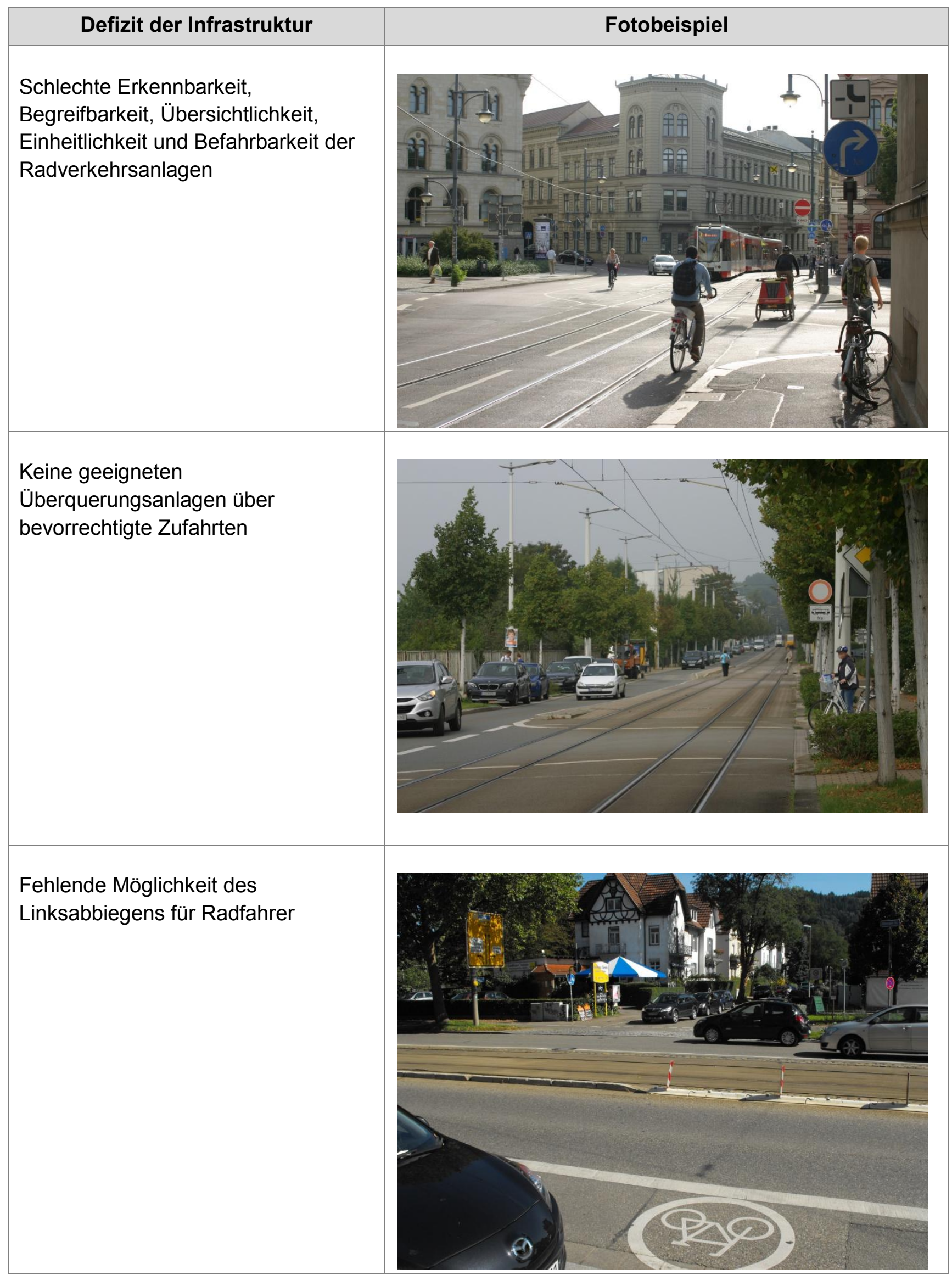




\section{Kraftfahrzeuge}

(Knotenpunkte ohne Lichtsignalanlage)

\begin{tabular}{|l|l|l|c|}
\hline \multicolumn{1}{|c|}{ Defizit der Infrastruktur } & Fotobeispiel \\
Fehlende Signalisierung trotz \\
mangelnder Erkennbarkeit, \\
Begreifbarkeit, Übersichtlichkeit, \\
Einheitlichkeit und/oder einer \\
relevanter Knotenstrombelastung
\end{tabular}


Besonderheiten an Haltestellen

(Knotenpunkte ohne Lichtsignalanlage)

keine Auffälligkeiten 


\section{A.8.1.3 Kreisverkehrsplätze}

\section{Fußgänger}

(Kreisverkehrsplätze)

\begin{tabular}{|l|l|l|l|}
\hline \multicolumn{1}{|c|}{ Defizit der Infrastruktur } \\
Überquerungsstellen zu weit von der \\
Kreisfahrbahn abgesetzt
\end{tabular}




\section{Radfahrer}

(Kreisverkehrsplätze)

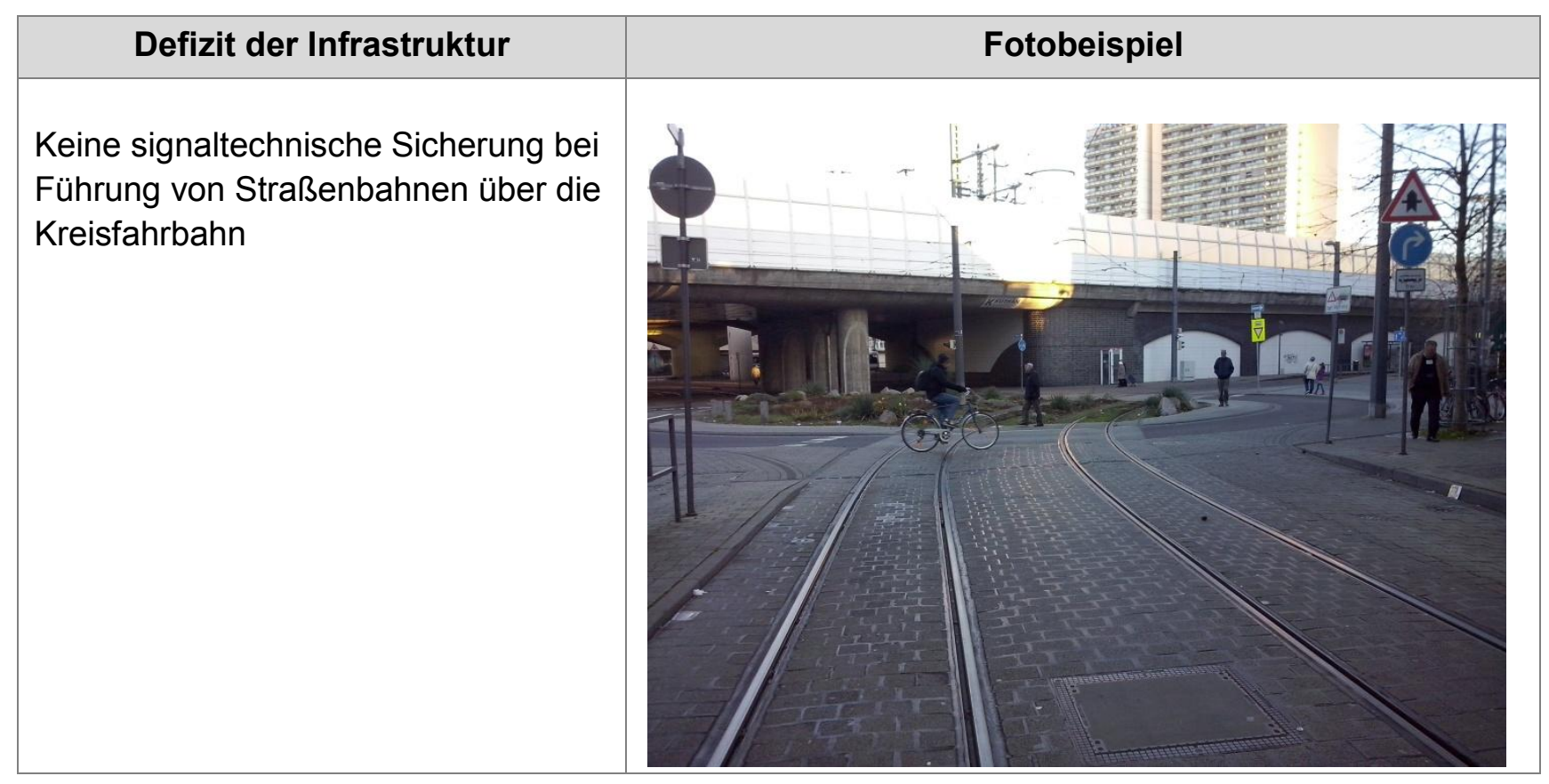




\section{Kraftfahrzeuge}

(Kreisverkehrsplätze)

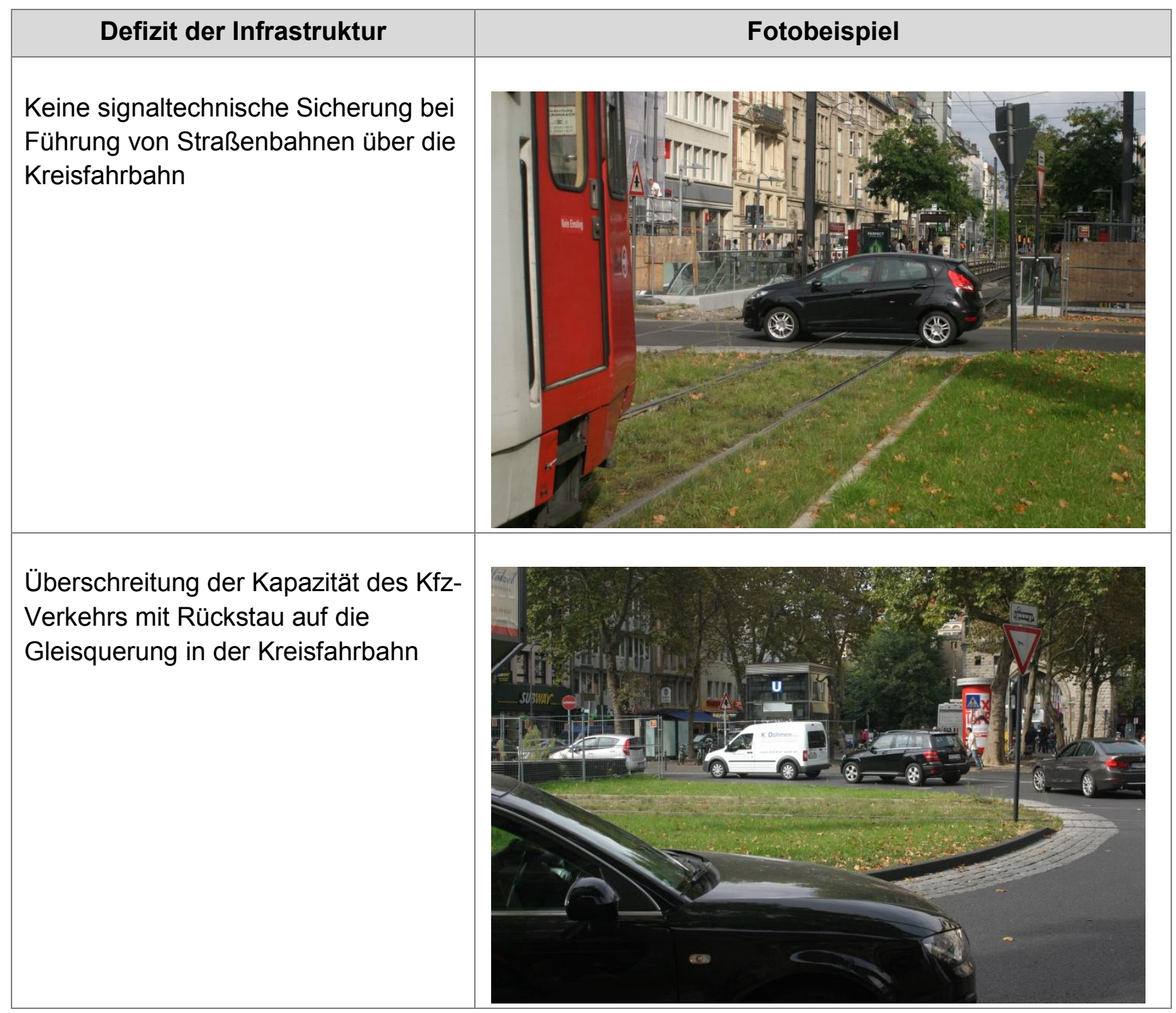




\section{Besonderheiten an Haltestellen}

(Kreisverkehrsplätze)

keine Auffälligkeiten 


\section{A.8.2 Streckenabschnitte zwischen Knotenpunkten nach Lage des Bahnkörpers}

\section{A.8.2.1 besonderer Bahnkörper in Mittellage}

\section{Fußgänger}

(besonderer Bahnkörper in Mittellage)

\begin{tabular}{|l|l|l|l|}
\hline \multicolumn{1}{|c|}{ Defizit der Infrastruktur } \\
Unzureichende Aufstellflächen \\
zwischen Gleisbereich und Fahrbahn \\
bei linearem Überquerungsbedarf
\end{tabular}




\begin{tabular}{l} 
Bei Signalregelung im Gleisbereich \\
keine Unabhängigkeit von der \\
Signalisierung der Fahrbahnfurten \\
\\
$\begin{array}{l}\text { Unterschiedliche Freigabezeit } \\
\text { hintereinanderliegender Furten }\end{array}$ \\
\hline $\begin{array}{l}\text { Keine eindeutige Signalisierung der } \\
\text { Straßenbahn in Verbindung mit den } \\
\text { Signalbildern F0 (Halt) und F1 (Fahrt } \\
\text { freigegeben) an signalisierten } \\
\text { Querungsstellen }\end{array}$ \\
\hline $\begin{array}{l}\text { Lange Wartezeiten/ Sperrzeiten für } \\
\text { Fußgänger }\end{array}$ \\
\hline
\end{tabular}




\section{Radfahrer}

(besonderer Bahnkörper in Mittellage)

Besonderer Bahnkörper von
Straßenbahnen ist zu Lasten
ausreichender Flächen für andere
Nutzungsansprüche
(betrifft insbesondere Radverkehr und
Fußgängerverkehr)

Keine Verhinderung der Mitbenutzung
von ÖPNV-Fahrstreifen mit Gleisen
durch Radfahrer
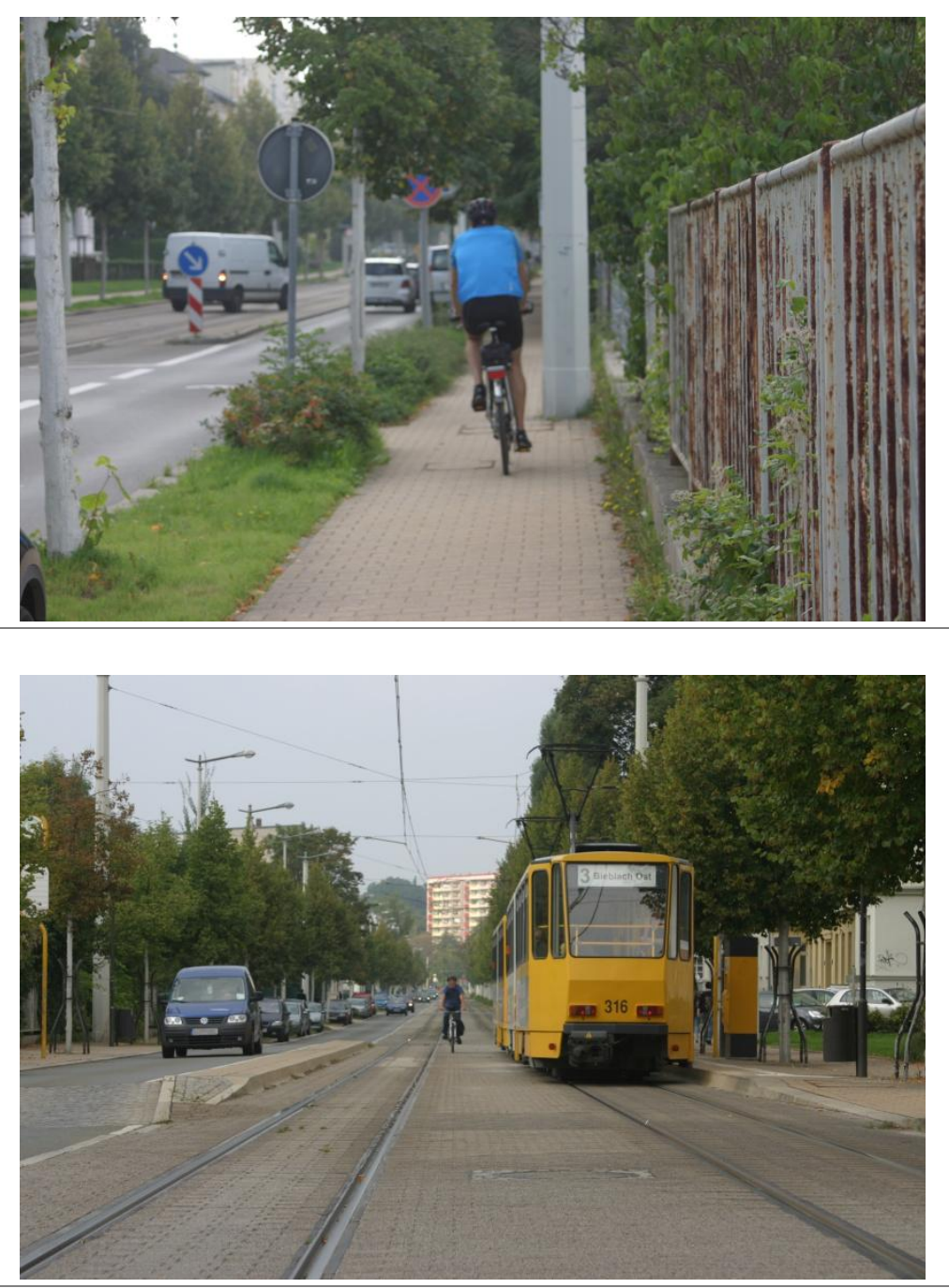


\section{Kraftfahrzeuge}

(besonderer Bahnkörper in Mittellage)

\begin{tabular}{|l|l|}
\hline \multicolumn{1}{|c|}{ Defizit der Infrastruktur } & \multicolumn{1}{|c|}{ Fotobeispiel } \\
\hline $\begin{array}{l}\text { Besondere Bahnkörper sind nicht } \\
\text { baulich durch Borde von der } \\
\text { Fahrbahn abgegrenzt }\end{array}$ &
\end{tabular}




\section{Besonderheiten an Haltestellen}

(besonderer Bahnkörper in Mittellage)

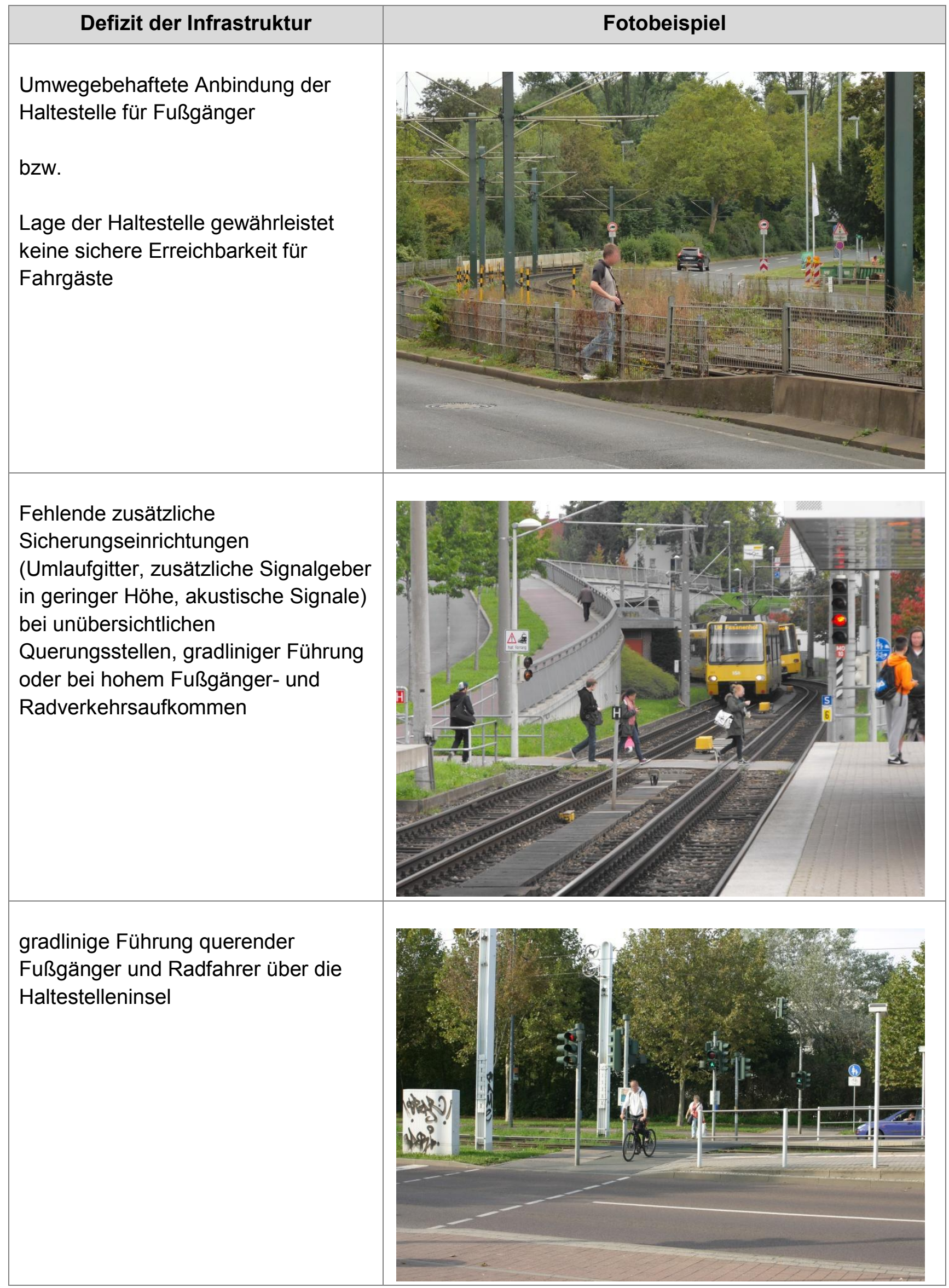


Freigabezeiten der Fußgängerfurten als Haltestellenzugang nicht auf einfahrende ÖPNV-Fahrzeuge abgestimmt

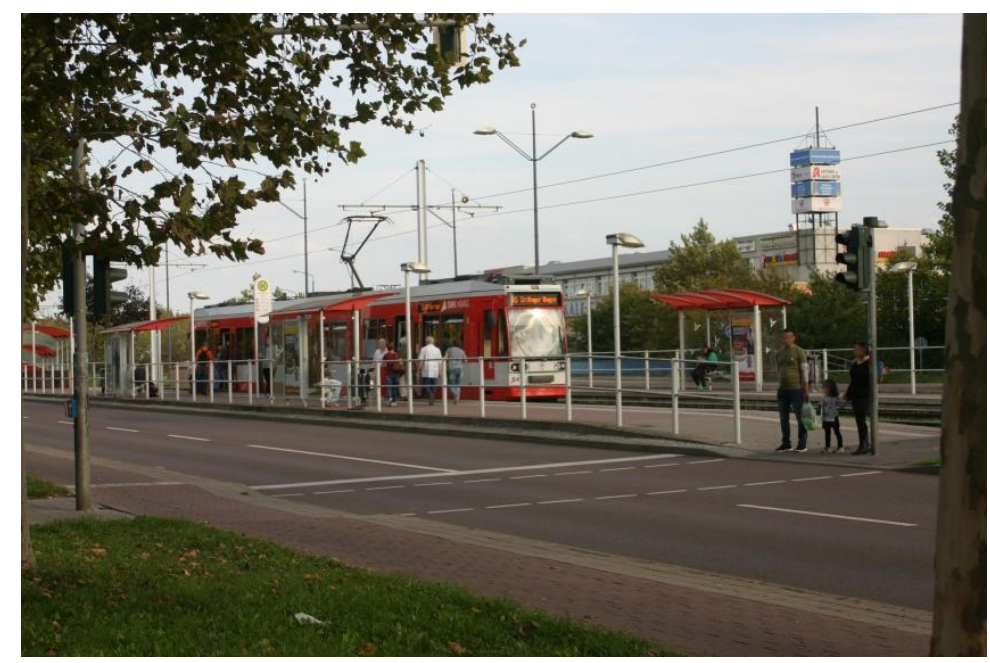

Keine Anrampung der Haltestelleninsel zur Überquerungsstelle

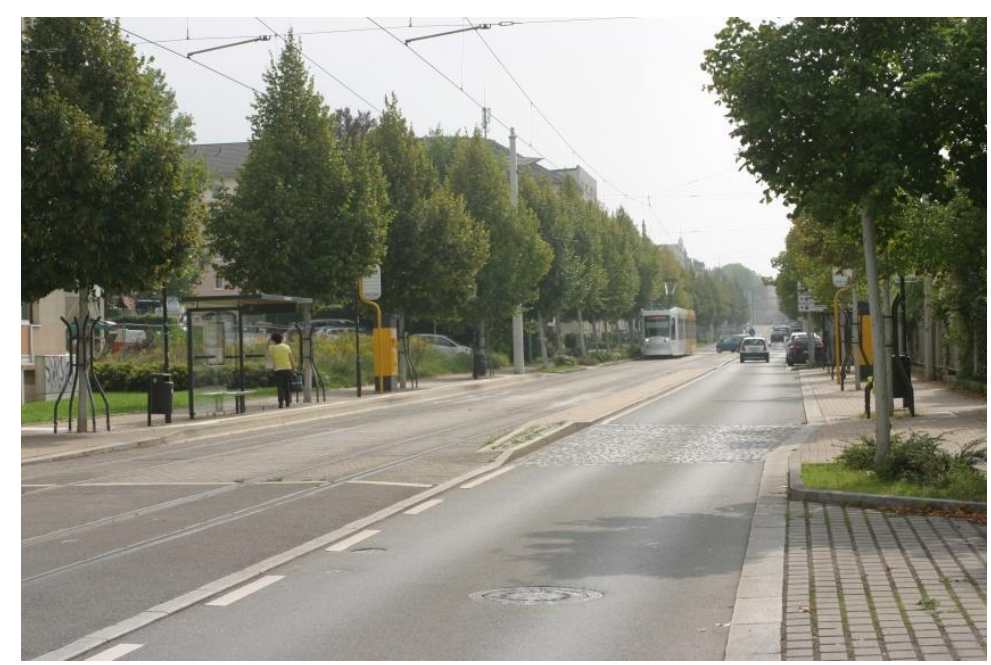




\section{A.8.2.2 Bahnkörper in Seitenlage}

\section{Fußgänger}

(Bahnkörper in Seitenlage)

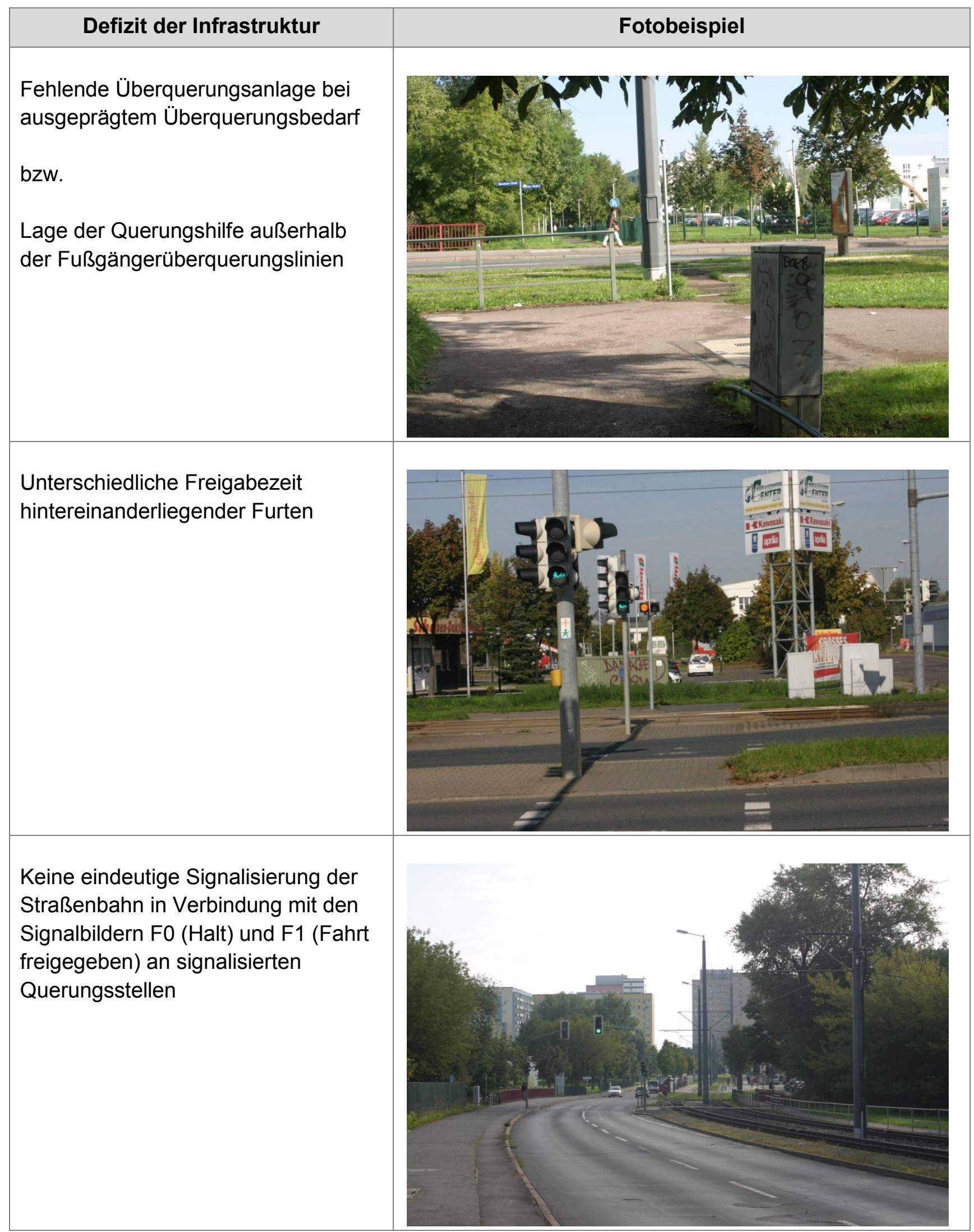




\section{Radfahrer}

(Bahnkörper in Seitenlage)






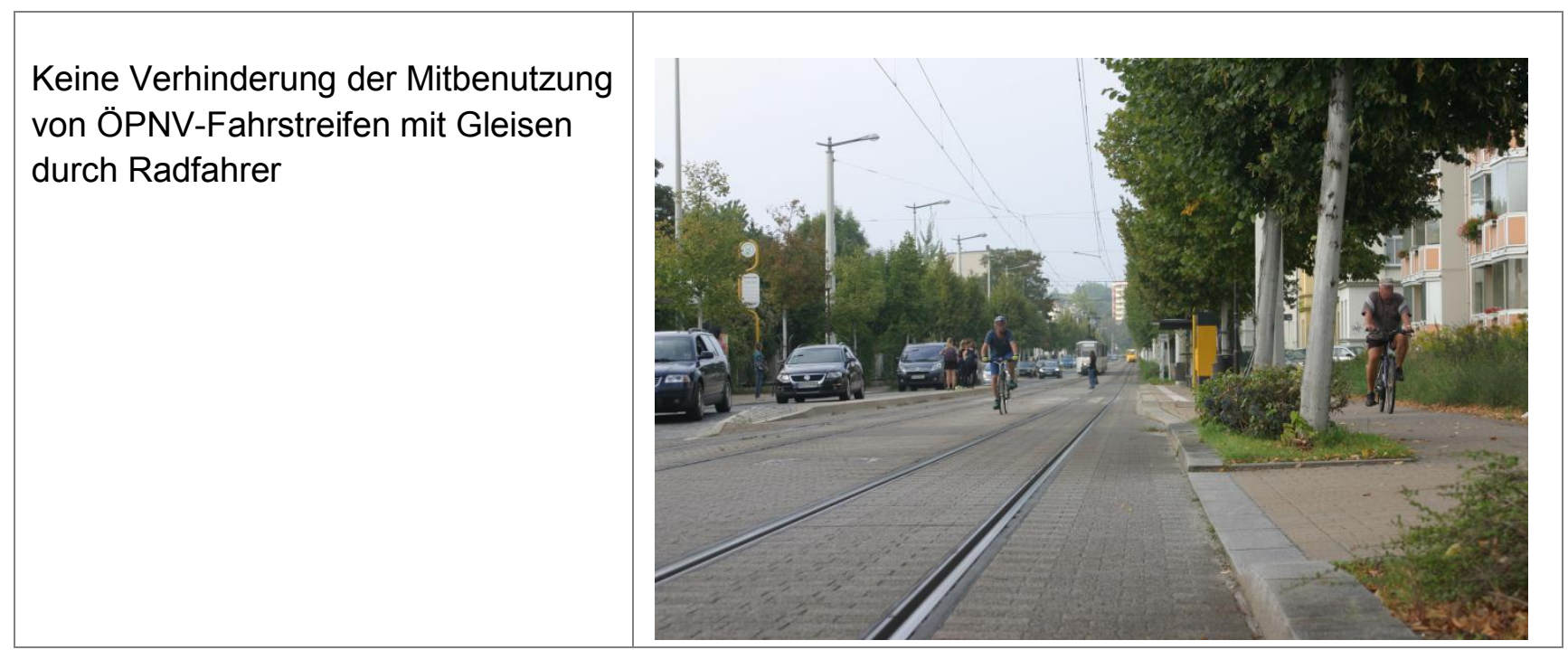




\section{Kraftfahrzeuge}

(Bahnkörper in Seitenlage)

keine Auffälligkeiten 


\section{Besonderheiten an Haltestellen}

(Bahnkörper in Seitenlage)

\begin{tabular}{|l|l|l|}
\hline \multicolumn{1}{|c|}{ Defizit der Infrastruktur } & \multicolumn{2}{|c|}{ Fotobeispiel } \\
\hline $\begin{array}{l}\text { Freigabezeiten der Fußgängerfurten } \\
\text { als Haltestellenzugang nicht auf } \\
\text { einfahrende ÖPNV-Fahrzeuge } \\
\text { abgestimmt }\end{array}$ &
\end{tabular}




\section{A.8.2.3 Straßenbündige Bahnkörper}

\section{Fußgänger}

(Straßenbündige Bahnkörper)

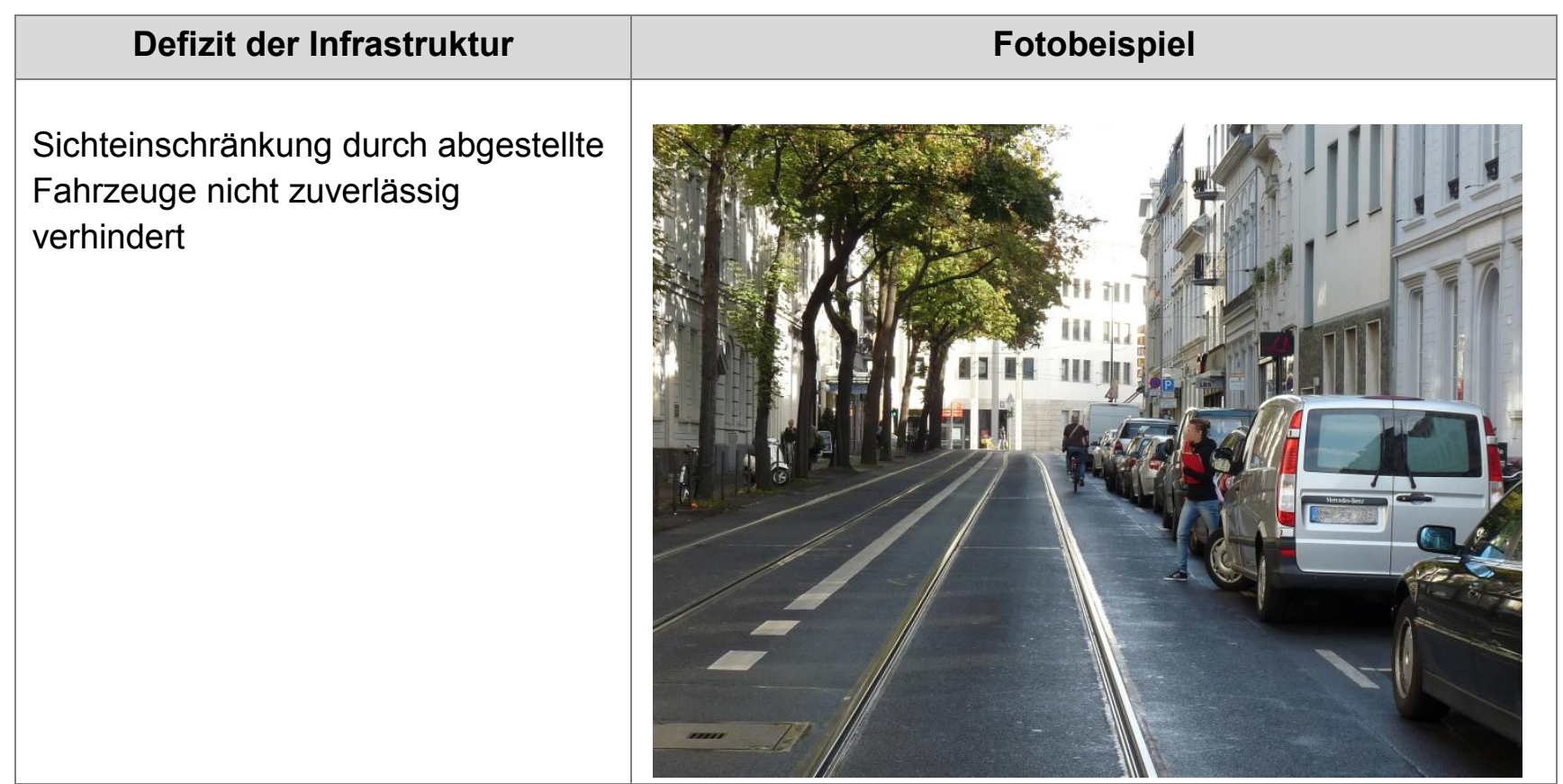




\section{Radfahrer}

(Straßenbündige Bahnkörper)

keine Auffälligkeiten 


\section{Kraftfahrzeuge}

(Straßenbündige Bahnkörper)

keine Auffälligkeiten 
Besonderheiten an Haltestellen

(Straßenbündige Bahnkörper)

keine Auffälligkeiten 
A.8.2.4 Unabhängige Bahnkörper

\section{Fußgänger}

(Unabhängige Bahnkörper)

\begin{tabular}{|l|l|}
\hline \multicolumn{1}{|c|}{ Defizit der Infrastruktur } & \multicolumn{1}{|c|}{ Fotobeispiel } \\
\hline $\begin{array}{l}\text { Fehlende Überquerungsanlage bei } \\
\text { ausgeprägtem Überquerungsbedarf }\end{array}$ & Ohne Abbildung \\
\hline $\begin{array}{l}\text { Lage der Querungshilfe außerhalb } \\
\text { der Fußgängerüberquerungslinien }\end{array}$ & Ohne Abbildung \\
\hline $\begin{array}{l}\text { Das Queren wird an nicht dafür } \\
\text { vorgesehenen Stellen nicht } \\
\text { ausreichend wirksam unterbunden }\end{array}$ & Ohne Abbildung \\
\hline
\end{tabular}




\section{Radfahrer}

(Unabhängige Bahnkörper)

\begin{tabular}{|l|l|}
\hline \multicolumn{1}{|c|}{ Defizit der Infrastruktur } & \multicolumn{1}{c|}{ Fotobeispiel } \\
\hline Fehlende zusätzliche & \\
Sicherungseinrichtungen & \\
(Umlaufgitter, zusätzliche Signalgeber & \\
in geringer Höhe, akustische Signale) & \\
bei unübersichtlichen & \\
Querungsstellen, gradliniger Führung & \\
oder bei hohem Fußgänger- und & \\
Radverkehrsaufkommen & \\
\hline
\end{tabular}




\section{Kraftfahrzeuge}

(Unabhängige Bahnkörper)

keine Auffälligkeiten 


\section{Besonderheiten an Haltestellen}

(Unabhängige Bahnkörper)

keine Auffälligkeiten

\section{Straßenbahn}

(Unabhängige Bahnkörper)

\begin{tabular}{|l|l|l|}
\hline \multicolumn{1}{|c|}{ Defizit der Infrastruktur } & \multicolumn{2}{c|}{ Fotobeispiel } \\
\hline $\begin{array}{l}\text { Fehlende Durchsetzung der } \\
\text { zulässigen Höchstgeschwindigkeit für } \\
\text { Straßenbahnfahrzeuge }\end{array}$ & Ohne Abbildung \\
\hline Geringe Sichtweiten & & \\
\hline
\end{tabular}




\section{A.9 ESAS-Defizitcheckliste mit ergänzten Checkpunkten für Straßenbahninfrastrukturen}

Die Defizitcheckliste stellt eine Ergänzung der durch BAIER ${ }^{136}$ im Rahmen der Fortschreibung der ESAS erstellten Prüfpunkte für Innerortsstraßen dar. Die grau hinterlegten Checkpunkte mit Bezug zu Straßenbahnverkehr sind daraus übernommen. Die Checkpunkte in schwarzer Schrift stellen Vorschläge für eine sinnvolle Erweiterung dar.

\section{Strecke}

\section{Fahrbahnen}

Grundsätzliche Überlegungen

- Besonderer Bahnkörper von Straßenbahnen ist zu Lasten ausreichender Flächen für andere Nutzungsansprüche

\section{Borde und Rinnen}

Borde

- Besondere Bahnkörper sind nicht baulich durch Borde von der Fahrbahn abgegrenzt

\section{Park- und Ladeflächen im Straßenraum}

Park- und Ladeflächen auf Streifen und in Buchten

- Senkrechtparkstände an Fahrstreifen mit Straßenbahnen

\section{Überquerung von Fahrbahnen durch Fußgänger}

Grundsätzliche Überlegungen und Einsatzbereiche

- Sichteinschränkung durch abgestellte Fahrzeuge nicht zuverlässig verhindert

- Fehlende Überquerungsanlage bei ausgeprägtem Überquerungsbedarf oder schutzbedürftigen Fußgängern

- Fehlende Überquerungsanlage bei hoher Verkehrsstärke/ zulässiger Höchstgeschwindigkeit

- Lage der Querungshilfe außerhalb der Fußgängerüberquerungslinien

Furten

- Grundsatz des durchgängigen Betriebs Tag und Nacht nicht beachtet

Überquerungsstellen an besonderen Bahnkörpern

- Keine Ausbildung der beidseitigen Aufstellflächen in Z-Form (auch an 
Überquerungsstellen als Haltestellenzugang)

- Unzureichende nutzbare Tiefe (besser: unzureichende Aufstellflächen zwischen Gleisbereich und Fahrbahn bei unsignalisierten Querungsstellen oder getrennter Signalisierung von Fahrbahn und Gleisquerung)

- Bei Signalregelung im Gleisbereich keine Unabhängigkeit von der Signalisierung der Fahrbahnfurten

- Unzureichende Sichtbeziehungen zwischen Überquerenden und Nahverkehrsfahrzeugen

- Freigabezeiten der Fußgängerfurten als Haltestellenzugang nicht auf einfahrende ÖPNV-Fahrzeuge abgestimmt

- unterschiedliche Freigabezeit hintereinanderliegender Furten

- Keine eindeutige Signalisierung der Straßenbahn in Verbindung mit den Signalbildern F0 (Halt) und F1 (Fahrt freigegeben) an signalisierten Querungsstellen

- Fehlende zusätzliche Sicherungseinrichtungen (Umlaufgitter, zusätzliche Signalgeber in geringer Höhe, akustische Signale) bei unübersichtlichen Querungsstellen, gradliniger Führung oder bei hohem Fußgänger- und Radverkehrsaufkommen

- Rot-Nachlauf nach Durchfahrt der Straßenbahn

- unzureichende Aufstellflächen zwischen Gleisbereich und Fahrbahn bei linearem Überquerungsbedarf

\section{Überquerungsanlagen für Radfahrer}

Überquerungsstellen an besonderen Bahnkörpern

- Keine Berücksichtigung des Radverkehrsaufkommens und besonderer Fahrzeugarten bei der Begegnung

- Keine Ausbildung der beidseitigen Aufstelfflächen in Z-Form angepasst an das Radverkehrsaufkommen

- Keine Berücksichtigung größerer freizuhaltender Sichtfelder

- Querung des Gleisbereichs nicht ungefähr rechtwinklig

- fehlende Signalisierung oder fehlende Umlaufgitter von Querungsstellen für den Radverkehr außerhalb von Knotenpunkten z.B. im Zuge von Radverkehrsachsen

\section{Anlagen des Öffentlichen Personenverkehrs}

Grundsätzliche Überlegungen

- Kein besonderer Bahnkörper bei mehr als zwei Fahrstreifen je Richtung

- Kein besonderer Bahnkörper in Einbahnstraßen mit gegenläufigem Straßenbahnverkehr 
- Kein besonderer Bahnkörper bei Schnellverkehr der Straßenbahn

- Unsicherer Verkehrsablauf auf ÖPNV-Fahrstreifen in Mittellage durch querenden Verkehr (Kfz, Rad, Fußgänger)

- Keine geeignete Gestaltung und fehlender Signalschutz am Ende der Mittellage

\section{Gemeinsame Führung von Straßenbahn und Kraftfahrzeugverkehr}

- Missbräuchliche Nutzung nicht zuverlässig ausgeschlossen

\section{Sonderfahrstreifen für Straßenbahn}

- Fehlende Erweiterung des Querschnitts bei Mitbenutzung durch Linienbusse

- Keine Gewährleistung der Bündelung der Überquerungen von Fußgängern und Radfahrern auf definierte Überquerungsstellen bei Bahnkörpern mit geschottertem Oberbau

- Fehlende Haltesichtweite bei Straßenbahnfahrzeugen mit Fahrt auf Sicht

- Fehlende Durchsetzung der zulässigen Höchstgeschwindigkeit für Straßenbahnfahrzeuge

\section{Mitbenutzung durch andere Verkehrsmittel}

- Keine Verhinderung der Mitbenutzung von ÖPNV-Fahrstreifen mit Gleisen durch Radfahrer

\section{Lage der Haltestellen im Straßenverlauf}

- Lage der Haltestelle gewährleistet keine sichere Erreichbarkeit für Fahrgäste

- Keine/ eingeschränkte Sichtverhältnisse für alle Verkehrsteilnehmer

- Keine Minimierung der Überquerungen von Fahrbahnen und Gleisen bei der Anordnung von Umsteigehaltestellen

\section{Haltestellen für Straßenbahnen}

- Keine nachvollziehbare Auswahl der Haltestellenform in Mittellage

- Keine erforderliche Abgrenzung der Wartefläche zur Fahrbahn bei Seitenbahnsteigen

- Keine bauliche/optische Abgrenzung von Verkehrsfläche und Fahrgastfläche

- Keine ausreichende Kennzeichnung von Inselköpfen der Haltestellenflächen und des Beginns besonderer Bahnkörper

- Keine Anrampung der Haltestelleninsel zur Überquerungsstelle 
- Unterschreitung der notwendigen Breiten der Haltestelleninsel

- Nichtbeachtung der zusätzlichen Ausstattung der Haltestelle unter Berücksichtigung der Barrierefreiheit

- Zugänge nicht an jeweils beiden Bahnsteigenden der Haltestelleninsel

- Umwegebehaftete Anbindung der Haltestelle für Fußgänger

- Fehlende signaltechnische Sicherung gegenüber Kraftfahrzeugen (Zeitinsel) bei Inselbahnsteigen mit Warteflächen am äußeren Fahrbahnrand

- gradlinige Führung querender Fußgänger und Radfahrer über die Haltestelleninsel

- Fehlende geschwindigkeitsdämpfende Elemente für Radfahrer an Rampen von Hochbahnsteigen

\section{Knotenpunkte}

\section{Allgemeines}

Allgemein

- unzureichende Anzahl an zulässigen Wendemöglichkeiten bei baulich getrennten Bahnkörpern mit anschließendem Verbot des Wendens oder Abbiegens am Knotenpunkt

\section{Einmündung/ Kreuzung mit Rechts-vor-Links-Regelung}

Allgemein

- Rechts-vor-Links-Regelung bei Einmündung/ Kreuzung mit Straßenbahnverkehr

\section{Einmündung/ Kreuzung mit vorfahrtregelnden Verkehrszeichen}

Allgemein

- Vorfahrtregelnden Verkehrszeichen bei Einmündung/ Kreuzung mit Straßenbahnverkehr und unzureichenden Sichtfeldern oder einer täglichen Verkehrsstärke von mehr als 100 Kraftfahrzeugen

Führung des Fußgängerverkehrs

- Keine geeigneten Überquerungsanlagen über bevorrechtige Zufahrten

Führung des Radverkehrs

- Keine geeigneten Überquerungsanlagen über bevorrechtige Zufahrten 


\section{Einmündung/ Kreuzung mit Lichtsignalanlagen}

Allgemein

- Eingeschränkte Sicht auf Signalgeber

- Unvollständige Signalisierung eines Knotenpunkts

- Grundsatz des durchgängigen Betriebs Tag und Nacht nicht beachtet

- Fehlender Zeitvorsprung oder räumlicher Vorsprung für Straßenbahnen im Fall von Missachtung von Fahrtrichtungsgeboten

- Fehlende Wiederholung des Richtungssignals über der Fahrbahn für KfzAbbiegestreifen über parallel geführte besondere Bahnkörper

- Fehlende Sichtfelder im Fall von Ausfall der Lichtsignalanlage und zur Erhöhung der Wahrnehmbarkeit bevorrechtigter Schienenfahrzeuge

- fehlende Erkennbarkeit von signalisierten Gleisquerungen außerhalb von Verkehrsknotenpunkten

- Keine/ ungeeignete Unterstützung von Fahrtrichtungsgeboten

Führung und Signalisierung des Fußgängerverkehrs

- Keine umwegefreie geradlinige Führung über Fußgängerfurten in jedem Knotenpunktarm

- Unterschiedliche Freigabezeit hintereinanderliegender Furten

Führung und Signalisierung des Radverkehrs

- Fehlende zusätzliche Signalgeber für den Radverkehr bei Führung des Radverkehrs auf der Fahrbahn und gemeinsamer Signalisierung mit dem KfzVerkehr

\section{Kreisverkehre}

Knotenpunktzu- und ausfahrten

- Einheitlichkeit der Knotenpunktzu- und Ausfahrten ist nicht gegeben

Führung des Fußgängerverkehrs

- Überquerungsstellen zu weit von der Kreisfahrbahn abgesetzt

Führung des Öffentlichen Personennahverkehrs

- Keine signaltechnische Sicherung bei Führung von Straßenbahnen über die Kreisinsel

- Überschreitung der Kapazität des Kfz-Verkehrs mit Rückstau auf die Gleisquerung 
in der Kreisfahrbahn

\section{Sonderformen}

Einmündung von Wohnwegen und Grundstückszufahrten

- Fehlende signaltechnische Sicherung bei Einmündung von Wohnwegen und Grundstückszufahrten mit kreuzendem Straßenbahnverkehr und unzureichenden Sichtfeldern oder einer täglichen Verkehrsstärke von mehr als 100 Kraftfahrzeugen 


\section{GDV}

DIE DEUTSCHEN VERSICHERER

Gesamtverband der Deutschen Versicherungswirtschaft e.V.

Wilhelmstraße 43/43G, 10117 Berlin

Postfach 0802 64, 10002 Berlin

Tel.: 030/20 20 - 50 00, Fax: 030/2020-6000

www.gdv.de, www.udv.de 